\title{
UAB
}

Universitat Autònoma de Barcelona

\section{Liouville-Weyl derivatives, best approximations, and moduli of smoothness}

Sergey Tikhonov

ADVERTIMENT. L'accés als continguts d'aquesta tesi queda condicionat a l'acceptació de les condicions d'ús establertes per la següent llicència Creative Commons:

(c) (1) $(9)$ http://cat.creativecommons.org/?page_id=184

ADVERTENCIA. El acceso a los contenidos de esta tesis queda condicionado a la aceptación de las condiciones de uso establecidas por la siguiente licencia Creative Commons: (c) (i) @ $\Theta$ http://es.creativecommons.org/blog/licencias/

WARNING. The access to the contents of this doctoral thesis it is limited to the acceptance of the use conditions set by the following Creative Commons license: (c) (i) \&) https://creativecommons.org/licenses/?lang=en 


\title{
Liouville-Weyl derivatives, best approximations, and moduli of smoothness
}

Ainur Jumabayeva

Director: Sergey Tikhonov

\section{UAB \\ Universitat Autònoma de Barcelona}

\author{
Doctorat en Matemàtiques \\ Universitat Autònoma de Barcelona \\ Departament de Matemàtiques \\ Febrer 2018
}





\section{Contents}

$\begin{array}{ll}\text { Acknowledgements } & 3\end{array}$

Introduction $\quad 5$

1 Liouville-Weyl derivatives, best approximations, and moduli of smoothness. $\left(L_{p}, L_{p}\right)$ inequalities 13

1.1 Notation . . . . . . . . . . . . . . . . . . 13

1.2 Moduli of smoothness _ . . . . . . . . . . . . 15

1.3 History of the question . . . . . . . . . . 17

1.4 General monotone sequences and their properties . . . . . 23

1.5 Auxiliary results . . . . . . . . . . . . . . . . . . 29

1.6 Estimates of best approximations and moduli of smoothness for generalized Liouville - Weyl derivatives . . . . 32

1.7 Estimates for transformed Fourier series in the limiting cases $p=1$ and $p=\infty \ldots \ldots \ldots \ldots$

1.8 Estimates from below of best approximations for generalized Liouville - Weyl derivatives _. . . . . . . . 46

2 Sharp Ul'yanov inequalities for Liouville-Weyl derivatives. $\left(L_{p}, L_{q}\right)$ inequalities $\quad 52$

2.1 Ul'yanov type inequalities for moduli of smoothness . . . . 52

2.2 Inequalities of different metrics for the best approximations 55

2.3 Some necessary lemmas . . . . . . . . . . . 56

2.4 New results in the case $1<p<q<\infty \ldots \ldots \ldots 7$

2.5 New results in the case $p=1<q<\infty \ldots \ldots$. . . . . 60

2.6 New results in the case $1<p<q=\infty \ldots \ldots \ldots$

2.7 New results in the case $1=p, q=\infty \ldots \ldots \ldots$

2.8 Estimates for the best approximations $\ldots \ldots \ldots \ldots$ 
3 Liouville-Weyl derivatives of double trigonometric series $\quad \mathbf{7 6}$

3.1 Some notations, known results, and goals . . . . . . . 76

3.2 Auxiliary results . . . . . . . . . . . . . . . . . 79

3.3 Upper estimates of angle best approximations of generalized Liouville-Weyl derivatives . . . . . . . . . . . 83

3.4 Estimates from below of angle best approximations of generalized Liouville-Weyl derivatives . . . . . . . . . . . 98

\section{Bibliography}




\section{Acknowledgements}

I would like to offer my special thanks to my supervisor Prof. Sergey Tikhonov for guiding me in this fascinating field of mathematics, for giving encouragement and support in times of need and advice he has provided throughout my time as his student. His careful editing contributed enormously to the production of this thesis.

I would like to thank Prof. Nazerke Tleukhanova and Prof. Erlan Nursultanov for generously sharing their valuable knowledge in the field with me and for their constant support during my studies. I am also grateful to Prof. Boris Simonov for many advice related to this work.

Moreover, I thank everyone at Centre de Recerca Matemàtica for their friendly attitude to me and for the inspiring atmosphere, especially Alberto Debernardi Pinos for helping me with many practical things.

I would also like to thank my parents for their wise counsel and support. I am grateful to my husband Serik, who have provided me through moral and emotional support in my life. 


\section{Introduction}

This work is devoted to the study of upper and lower estimates of norms and best approximations of the generalized Liouville-Weyl derivatives via the best approximations of functions themselves. We also study estimates of moduli of smoothness of the generalized Liouville-Weyl derivatives via moduli of smoothness of functions themselves.

Let the series

$$
\frac{a_{0}(f)}{2}+\sum_{\nu=1}^{\infty}\left(a_{\nu}(f) \cos \nu x+b_{\nu}(f) \sin \nu x\right)
$$

be the Fourier series of $f \in L_{1}$. The transformed Fourier series of (1.1.1) is given by

$$
\sigma(f, \lambda, \beta):=\sum_{\nu=1}^{\infty} \lambda_{\nu}\left[a_{\nu} \cos \left(\nu x+\frac{\pi \beta}{2}\right)+b_{\nu} \sin \left(\nu x+\frac{\pi \beta}{2}\right)\right],
$$

where $\beta \in \mathbb{R}$ and $\lambda=\left\{\lambda_{n}\right\}$ is a sequence of positive numbers.

We call the function $\varphi(x) \sim \sigma(f, \lambda, \beta)$ the Liouville-Weyl derivative (or $(\lambda, \beta)$-derivative of the function $f)$ and denote it by $f^{(\lambda, \beta)}$. As an important example, for $\lambda_{n}=n^{r}, r>0, \beta=r$, we have $f^{(\lambda, \beta)}=f^{(r)}$ and for $\lambda_{n}=n^{r}, r>$ $0, \beta=r+1$ we have $f^{(\lambda, \beta)}= \pm \tilde{f}^{(r)}$, where $f^{(r)}$ is the fractional derivative in the sense of Weyl and $\tilde{f}^{(r)}$ is the $r$-fractional derivative of the conjugate function $\tilde{f}$. For more historical details concerning the Liouville-Weyl derivatives, see the papers [7], [22], [38],[55], [73].

Let $E_{n}(f)_{p}$ be the best approximation of a function $f \in L_{p}$ by trigonometric polynomials of degree at most $n$, i.e.,

$$
E_{n}(f)_{p}=\inf _{\alpha_{k}, \beta_{k} \in \mathbb{R}}\left\|f(x)-\sum_{k=0}^{n}\left(\alpha_{k} \cos k x+\beta_{k} \sin k x\right)\right\|_{p} .
$$

Denote by $\omega_{\alpha}(f, \delta)_{p}$ the modulus of smoothness of fractional order $\alpha, \alpha>0$, of the function $f \in L_{p}$, i.e.,

$$
\omega_{\alpha}(f, \delta)_{p}=\sup _{|h| \leqslant \delta}\left\|\Delta_{h}^{\alpha}(f)\right\|_{p}
$$

where

$$
\Delta_{h}^{\alpha}(f)=\sum_{\nu=0}^{\infty}(-1)^{\nu}\left(\begin{array}{l}
\alpha \\
\nu
\end{array}\right) f(x+(\alpha-\nu) h)
$$


is a difference of fractional order $\alpha>0$ of a function $f \in L_{p}$ at the point $x$ with increment $h$.

The study of the question of the existence of the $r$-th derivative of the function $f$ was started by Bernstein [4] in 1912. He proved the following result for $p=\infty$ (for $1 \leqslant p<\infty$, see [8]):

If $f \in L_{p}, 1 \leqslant p \leqslant \infty$, and $\sum_{k=0}^{\infty}(k+1)^{r-1} E_{k}(f)_{p}<\infty, r \in \mathbb{N}$, then

$$
\left\|f^{(r)}\right\|_{p} \leqslant C(r) \sum_{k=0}^{\infty}(k+1)^{r-1} E_{k}(f)_{p} .
$$

Later, Stechkin [60] obtained the following inequality for the best approximations of $f^{(r)}$ for $p=\infty$ (for $1 \leqslant p<\infty$, see [37]):

$$
E_{n}\left(f^{(r)}\right)_{p} \leqslant C(r, p)\left(n^{r} E_{n}(f)_{p}+\sum_{k=n+1}^{\infty} k^{r-1} E_{k}(f)_{p}\right), \quad r, n \in \mathbb{N} .
$$

The corresponding estimate for moduli of smoothness was obtained by Johnen and Scherer [29] (see also [8, pp.178-179]):

$$
\omega_{k}\left(f^{(r)}, \frac{1}{n}\right)_{p} \leqslant C(k, r, p) \sum_{\nu=n+1}^{\infty} \nu^{r-1} \omega_{k+r}\left(f, \frac{1}{\nu}\right)_{p}, 1 \leqslant p \leqslant \infty, r, k, n \in \mathbb{N} .
$$

These investigations have been further developed by Timan, Ditzian, Simonov, Potapov, Tikhonov and others.

In [54] and [55], the authors considered the generalized derivatives in the sense of Liouville-Weyl and extended mentioned above results as follows.

Theorem A1. Let $1<p<\infty, \theta=\min (2, p), \beta \in \mathbb{R}$, and $\lambda=\left\{\lambda_{n}\right\}_{n=1}^{\infty}$ be non-decreasing sequence of positive numbers satisfying $\Delta_{2}-$ condition, i.e., $\lambda_{2 n} \leqslant \lambda_{n}$. If $f \in L_{p}$ and

$$
\sum_{n=1}^{\infty}\left(\lambda_{n+1}^{\theta}-\lambda_{n}^{\theta}\right) E_{n}^{\theta}(f)_{p}<\infty
$$

then there exists a function $f^{(\lambda, \beta)} \in L_{p}$ with the Fourier series $\sigma(f, \lambda, \beta)$ and

$$
\left\|f^{(\lambda, \beta)}\right\|_{p} \lesssim\left\{\lambda_{1}^{\theta} E_{0}^{\theta}(f)_{p}+\sum_{n=1}^{\infty}\left(\lambda_{n+1}^{\theta}-\lambda_{n}^{\theta}\right) E_{n}^{\theta}(f)_{p}\right\}^{\frac{1}{\theta}}
$$




$$
E_{n}\left(f^{(\lambda, \beta)}\right) \lesssim\left\{\lambda_{n}^{\theta} E_{n}^{\theta}(f)_{p}+\sum_{k=n+1}^{\infty}\left(\lambda_{k+1}^{\theta}-\lambda_{k}^{\theta}\right) E_{k}^{\theta}(f)_{p}\right\}^{\frac{1}{\theta}} .
$$

Moreover, if $\alpha>0$ and $\left\{\frac{\lambda_{n}}{n^{r}}\right\}$ is decreasing for some $r>0$, then

$$
\begin{aligned}
\omega_{\alpha}\left(f^{(\lambda, \beta)}, \frac{1}{n}\right)_{p} \lesssim & \left\{n^{-\alpha \theta} \sum_{k=1}^{n}\left(\frac{\lambda_{k}^{\theta}}{k^{r \theta}}-\frac{\lambda_{k+1}^{\theta}}{(k+1)^{r \theta}}\right) k^{(r+\alpha) \theta} \omega_{\alpha+r}^{\theta}\left(f, \frac{1}{k}\right)_{p}\right. \\
& \left.+\sum_{k=n+2}^{\infty}\left(\lambda_{k+1}^{\theta}-\lambda_{k}^{\theta}\right) \omega_{\alpha+r}^{\theta}\left(f, \frac{1}{k}\right)_{p}+\lambda_{n+1}^{\theta} \omega_{\alpha+r}^{\theta}\left(f, \frac{1}{n+1}\right)_{p}\right\}^{\frac{1}{\theta}} .
\end{aligned}
$$

Moreover, the corresponding estimates in the limiting cases $(p=1, \infty)$ were also obtained. It was assumed in this case that $\left\{\lambda_{n}\right\}$ is convex or concave.

Our main goal in Section 1 is to extend results mentioned above for a larger class of the Liouville-Weyl derivatives. For this purpose we consider a more general class of sequences $\left\{\lambda_{n}\right\}$. We replace the monotonicity condition on $\left\{\lambda_{n}\right\}$ and $\left\{\Delta \lambda_{n}\right\}$ by general monotonicity.

Let us recall the definition of general monotone sequences $(G M)$ introduced in [69].

Definition 1.4.1 A sequence $\lambda:=\left\{\lambda_{n}\right\}_{n=1}^{\infty}$ of real numbers is said to be general monotone, written $\lambda \in G M$, if the relation

$$
\sum_{k=n}^{2 n}\left|\lambda_{k}-\lambda_{k+1}\right| \leqslant C\left|\lambda_{n}\right|
$$

holds for all integer $n$, where the constant $C$ is independent of $n$.

In subsection 1.6, we proved the following generalization of Theorem A1.

Theorem 1.6.1 Let $1<p<\infty, \theta=\min (2, p), \quad \lambda=\left\{\lambda_{n}\right\}_{n=1}^{\infty} \in G M$, $\alpha \in \mathbb{R}_{+}$, and $r \in \mathbb{R}_{+} \cup\{0\}$. If $f \in L_{p}$ and the series

$$
\sum_{n=1}^{\infty}\left|\lambda_{n+1}^{\theta}-\lambda_{n}^{\theta}\right| E_{n}^{\theta}(f)_{p}
$$

converges, then there exists a function $f^{(\lambda, \beta)} \in L_{p}$ with the Fourier series $\sigma(f, \lambda, \beta)$ and

$$
\left\|f^{(\lambda, \beta)}\right\|_{p} \lesssim\left\{\lambda_{1}^{\theta} E_{0}^{\theta}(f)_{p}+\sum_{n=1}^{\infty}\left|\lambda_{n+1}^{\theta}-\lambda_{n}^{\theta}\right| E_{n}^{\theta}(f)_{p}\right\}^{\frac{1}{\theta}},
$$




$$
E_{n}\left(f^{(\lambda, \beta)}\right)_{p} \lesssim\left\{\lambda_{[n / 2]}^{\theta} E_{[n / 2]}^{\theta}(f)_{p}+\sum_{k=[n / 4]}^{\infty}\left|\lambda_{k+1}^{\theta}-\lambda_{k}^{\theta}\right| E_{k}^{\theta}(f)_{p}\right\}^{\frac{1}{\theta}}
$$

and

$$
\begin{aligned}
\omega_{\alpha}\left(f^{(\lambda, \beta)}, \frac{1}{n}\right)_{p} \lesssim\left\{n^{-\alpha \theta} \sum_{k=1}^{n}\left|\frac{\lambda_{k}^{\theta}}{k^{r \theta}}-\frac{\lambda_{k+1}^{\theta}}{(k+1)^{r \theta}}\right| k^{(r+\alpha) \theta} \omega_{\alpha+r}^{\theta}\left(f, \frac{1}{k}\right)_{p}\right. \\
\left.+\sum_{k=n+1}^{\infty}\left|\lambda_{k+1}^{\theta}-\lambda_{k}^{\theta}\right| \omega_{\alpha+r}^{\theta}\left(f, \frac{1}{k}\right)_{p}+\lambda_{n}^{\theta} \omega_{\alpha+r}^{\theta}\left(f, \frac{1}{n}\right)_{p}\right\}^{\frac{1}{\theta}} .
\end{aligned}
$$

In subsection 1.7 we obtain the corresponding inequalities for $p=1$ and $p=\infty$.

In subsection 1.8 we study estimates from below of norms and best approximations of the generalized Liouville-Weyl derivatives. We obtain the following result.

Theorem 1.8.1 Let $1<p<\infty, \max (p, 2) \leqslant \tau<\infty, \lambda=\left\{\lambda_{n}\right\}_{n=1}^{\infty}$ be a sequence of positive numbers such that $\lambda \in G M$. Assume that $\left\{\lambda_{n}\right\}_{n=1}^{\infty}$ satisfies the additional condition

$$
\left(\sum_{k=1}^{n}\left|\lambda_{2^{k}}^{\tau}-\lambda_{2^{k-1}}^{\tau}\right|\right)^{\frac{1}{\tau}} \leqslant C\left|\lambda_{2^{n}}\right|
$$

for all integer $n$, where the constant $C$ is independent of $n$.

If for $f \in L_{p}$ there exists a function $f^{(\lambda, \beta)} \in L_{p}$, with the Fourier series $\sigma(f, \lambda, \beta)(\beta \in \mathbb{R})$, then

$$
\begin{aligned}
\left\|f^{(\lambda, \beta)}\right\|_{p} & \gtrsim\left\{\lambda_{1}^{\tau} E_{0}^{\tau}(f)_{p}+\sum_{\nu=1}^{\infty}\left|\lambda_{2^{\nu}}^{\tau}-\lambda_{2^{\nu-1}}^{\tau}\right| E_{2^{\nu}-1}^{\tau}(f)_{p}\right\}^{\frac{1}{\tau}}, \\
E_{2^{m}-1}\left(f^{(\lambda, \beta)}\right)_{p} & \gtrsim\left\{\lambda_{2^{m-1}}^{\tau} E_{2^{m}-1}^{\tau}(f)_{p}+\sum_{\nu=m}^{\infty}\left|\lambda_{2^{\nu}}^{\tau}-\lambda_{2^{\nu-1}}^{\tau}\right| E_{2^{\nu}-1}^{\tau}(f)_{p}\right\}^{\frac{1}{\tau}} .
\end{aligned}
$$

In the second section, we study sharp Ul'yanov-type inequalities for moduli of smoothness of fractional order. The $(p, q)$-inequalities between moduli of smoothness, nowadays called Ul'yanov-type inequalities. The first result of this type was obtained by Ul'yanov [85] in 1968: 


$$
\omega(f, \delta)_{q} \lesssim\left(\int_{0}^{\delta}\left(t^{-\theta} \omega(f, t)_{p}\right)^{q_{1}} \frac{d t}{t}\right)^{1 / q_{1}}
$$

where

$$
1 \leqslant p<q \leqslant \infty, \quad \theta=\frac{1}{p}-\frac{1}{q}, \quad q_{1}= \begin{cases}q, & q<\infty, \\ 1, & q=\infty .\end{cases}
$$

Here $\omega(f, \delta)_{p}=\omega_{1}(f, \delta)_{p}$ is the modulus of continuity and $\omega_{k}(f, \delta)_{p}$ is the modulus of smoothness of order $k \in \mathbb{N}$.

This result was extended by DeVore, Riemenschneider, and Sharpley for moduli of smoothness of an integer order and the $K$-functionals [9] (see also $[20,21])$. Similar estimates for moduli of smoothness of the derivatives of a function was obtained by Ditzian and Tikhonov. Recently, a sharp form of Ulyanov-type inequalities was extensively investigated by Simonov, Tikhonov, Trebels, among other authors [19, 34, 36, 56, 68, 83].

Our main goal is to improve results of Tikhonov and Trebels [73] by considering a more general class of sequences $\left\{\lambda_{n}\right\}$. We also consider all limiting cases separately:

(i) $p=1<q<\infty$;

(ii) $1<p<q=\infty$;

(iii) $p=1<q=\infty$.

In subsection 2.1 we survey some known results concerning Ul'yanovtype inequalities for moduli of smoothness. The corresponding result concerning inequalities of different metrics for the best approximations can be found in subsection 2.2. In subsection 2.3 we give some necessary lemmas. In subsection 2.4 we obtain the main results for non-limiting case. In particular, we obtain the following.

Theorem 2.4.1 Let $f \in L_{p}, 1<p<q<\infty, \theta=1 / p-1 / q$. Let $\lambda=\left\{\lambda_{n}\right\}_{n=1}^{\infty} \in G M$. Then, for any $\alpha>0$,

$$
\omega_{\alpha}\left(f^{(\lambda, \beta)}, \frac{1}{2^{n}}\right)_{q} \lesssim\left(\sum_{m=n}^{\infty}\left(2^{m(\theta+\rho)} \Lambda_{2^{m}} \omega_{\alpha+\rho+\theta}\left(f, \frac{1}{2^{m}}\right)_{p}\right)^{q}\right)^{1 / q}
$$

where

$$
\Lambda_{n}:=\max _{1 \leqslant k \leqslant n} \frac{\left|\lambda_{2^{k}}\right|}{2^{k \rho}}, \quad \rho>0 .
$$


In subsection 2.5 we obtain the sharp Ul'yanov-type inequalities for the first limiting case: $p=1<q<\infty$.

Theorem 2.5.1 Let $f \in L_{p}, 1=p<q<\infty, \theta=1-1 / q$. Let $\lambda=$ $\left\{\lambda_{n}\right\}_{n=1}^{\infty} \in G M$. Then, for any $\alpha>0$ and $0<\varepsilon \leqslant \min (\rho, \theta)$,

$$
\omega_{\alpha}\left(f^{(\lambda, \beta)}, \frac{1}{2^{n}}\right)_{q} \lesssim\left(\sum_{m=n}^{\infty}\left(2^{m(\theta+\rho)} \Lambda_{2^{m}} \omega_{\alpha+\rho+\theta-\varepsilon}\left(f, \frac{1}{2^{m}}\right)_{1}\right)^{q}\right)^{1 / q}
$$

where

$$
\Lambda_{2^{n}}:=2^{-n \frac{\varepsilon}{2}} \max _{1 \leqslant k \leqslant n} \frac{\left|\lambda_{2^{k}}\right|}{2^{k\left(\rho-\frac{\varepsilon}{2}\right)}}
$$

Theorem 2.5.2 Let $f \in L_{p}, 1=p<q<\infty, \theta=1-1 / q$, and $\lambda=$ $\left\{\lambda_{n}\right\}_{n=1}^{\infty} \in G M$. Then, for any $\alpha>0$,

$$
\omega_{\alpha}\left(f^{(\lambda, \beta)}, \frac{1}{2^{n}}\right)_{q} \lesssim\left(\sum_{m=n}^{\infty}\left(2^{m(\theta+\rho)} \Lambda_{2^{m}} \omega_{\alpha+\rho+\theta}\left(f, \frac{1}{2^{m}}\right)_{1}\right)^{q}\right)^{1 / q}
$$

where

$$
\Lambda_{N}:=\left(\sum_{k=1}^{N} \frac{\left|\lambda_{k}\right|^{q}}{k^{(q \rho+1)}}\right)^{\frac{1}{q}}, \quad \rho>0 .
$$

In subsections 2.6 and 2.7, we obtain the sharp Ul'yanov-type inequalities for the cases $1<p<q=\infty$ and $p=1, q=\infty, \theta=1$, correspondingly.

In subsection 2.8 we consider estimates for the $L_{q}$-best approximations of the generalized Liouville-Weyl derivatives via the $L_{p}$-best approximations of functions themselves.

The third section is devoted to the study of estimates of the angle best approximations of the generalized Liouville-Weyl derivatives by the angle approximation of functions themselves in two-dimensional case. We consider the generalized Liouville-Weyl derivatives in place of the classical Weyl mixed derivatives. Our main goal is to prove analogues of Theorems 1.6.1 and 1.8.1 mentioned above in two-dimensional case. In subsection 3.1 we give necessary notation and known results. Useful lemmas are given in subsection 3.2.

Let $Y_{m_{1}, m_{2}}(f)_{p}$ be the best approximation by a two-dimensional angle of the function $f \in L_{p}\left(\mathbb{T}^{2}\right)$, i.e., 


$$
Y_{m_{1}, m_{2}}(f)_{p}=\inf _{T_{m_{1}, \infty}, T_{\infty, m_{2}}}\left\|f-T_{m_{1}, \infty}-T_{\infty, m_{2}}\right\|_{p}
$$

where the function $T_{m_{1}, \infty}\left(x_{1}, x_{2}\right) \in L_{p}\left(\mathbb{T}^{2}\right)$ is a trigonometric polynomial of order at most $m_{1}$ in $x_{1}$, and the function $T_{\infty, m_{2}}\left(x_{1}, x_{2}\right) \in L_{p}\left(\mathbb{T}^{2}\right)$ is a trigonometric polynomial of order at most $m_{2}$ in $x_{2}$.

By $\sigma(f)$ we will denote the Fourier series of a function $f \in L_{p}\left(\mathbb{T}^{2}\right)$, that is

$$
\begin{array}{r}
\sigma(f) \equiv \sum_{n_{1}=0}^{\infty} \sum_{n_{2}=0}^{\infty}\left(a_{n_{1} n_{2}} \cos n_{1} x_{1} \cos n_{2} x_{2}+b_{n_{1} n_{2}} \cos n_{1} x_{1} \sin n_{2} x_{2}+\right. \\
\left.+c_{n_{1} n_{2}} \sin n_{1} x_{1} \cos n_{2} x_{2}+d_{n_{1} n_{2}} \sin n_{1} x_{1} \sin n_{2} x_{2}\right)
\end{array}
$$

where for the sake of brevity we set $\cos (0 \cdot t)=\frac{1}{2}$.

The transformed Fourier series of $\sigma(f)$ is given by

$$
\begin{aligned}
\sigma\left(f, \lambda, \beta_{1}, \beta_{2}\right) \equiv \sum_{n_{1}=1}^{\infty} \sum_{n_{2}=1}^{\infty} \lambda_{n_{1}, n_{2}} & \left(a_{n_{1} n_{2}} \cos \left(n_{1} x_{1}+\beta_{1} \pi / 2\right) \cos \left(n_{2} x_{2}+\beta_{2} \pi / 2\right)\right. \\
& +b_{n_{1} n_{2}} \cos \left(n_{1} x_{1}+\beta_{1} \pi / 2\right) \sin \left(n_{2} x_{2}+\beta_{2} \pi / 2\right) \\
& +c_{n_{1} n_{2}} \sin \left(n_{1} x_{1}+\beta_{1} \pi / 2\right) \cos \left(n_{2} x_{2}+\beta_{2} \pi / 2\right) \\
& \left.+d_{n_{1} n_{2}} \sin \left(n_{1} x_{1}+\beta_{1} \pi / 2\right) \sin \left(n_{2} x_{2}+\beta_{2} \pi / 2\right)\right),
\end{aligned}
$$

where $\beta_{1}, \beta_{2} \in \mathbb{R}$ and $\lambda=\left\{\lambda_{n_{1} n_{2}}\right\}_{n_{1}, n_{2} \in \mathbb{N}}$ is a sequence of real numbers.

By analogy with the one-dimensional case we call the function $\varphi\left(x_{1}, x_{2}\right) \sim \sigma\left(f, \lambda, \beta_{1}, \beta_{2}\right)$ the $\left(\lambda, \beta_{1}, \beta_{2}\right)-$ mixed derivative of the function $f$ (or Liouville-Weyl derivative) and denote it by $f^{\left(\lambda, \beta_{1}, \beta_{2}\right)}\left(x_{1}, x_{2}\right)$.

Definition 3.1.1 A sequence $\lambda=\left\{\lambda_{n_{1} n_{2}}\right\}_{n_{1}, n_{2} \in N}$ is said to be general monotone, written $\lambda \in G M^{2}$, if the relations

$$
\begin{aligned}
& \sum_{k_{1}=n_{1}}^{2 n_{1}}\left|\lambda_{k_{1}, n_{2}}-\lambda_{k_{1}+1, n_{2}}\right| \leqslant C\left|\lambda_{n_{1}, n_{2}}\right|, \\
& \sum_{k_{2}=n_{2}}^{2 n_{2}}\left|\lambda_{n_{1}, k_{2}}-\lambda_{n_{1}, k_{2}+1}\right| \leqslant C\left|\lambda_{n_{1}, n_{2}}\right|, \\
& \sum_{k_{1}=n_{1}}^{2 n_{1}} \sum_{k_{2}=n_{2}}^{2 n_{2}}\left|\lambda_{k_{1}, k_{2}}-\lambda_{k_{1}+1, k_{2}}-\lambda_{k_{1}, k_{2}+1}+\lambda_{k_{1}+1, k_{2}+1}\right| \leqslant C\left|\lambda_{n_{1}, n_{2}}\right|
\end{aligned}
$$


hold for all integers $n_{1}$ and $n_{2}$, where the constant $C$ is independent of $n_{1}$ and $n_{2}$.

In subsection 3.3 similarly to one-dimensional inequalities given by Theorem 1.6.1, we obtain estimates of the angle approximations of $\left(\lambda, \beta_{1}, \beta_{2}\right)$ derivatives by angle approximation of functions themselves.

Theorem 3.3.1 Let $1<p<\infty, 0<\theta \leqslant \min (p, 2), \lambda=\left\{\lambda_{n_{1}, n_{2}}\right\}_{n_{1}, n_{2} \in N}$ be a sequence of positive numbers such that $\lambda \in G M^{2}, \alpha_{i} \in \mathbb{R}_{+}, r_{i} \in \mathbb{R}_{+} \cup\{0\}$, and $\beta_{i} \in \mathbb{R}(i=1,2)$.

If for $f \in L_{p}^{0}\left(\mathbb{T}^{2}\right)$ the series

$$
\begin{aligned}
& \sum_{n_{1}=1}^{\infty}\left|\lambda_{n_{1}+1,1}^{\theta}-\lambda_{n_{1}, 1}^{\theta}\right| Y_{n_{1}, 0}^{\theta}(f)_{p}+\sum_{n_{2}=1}^{\infty}\left|\lambda_{1, n_{2}+1}^{\theta}-\lambda_{1, n_{2}}^{\theta}\right| Y_{0, n_{2}}^{\theta}(f)_{p} \\
& +\sum_{k_{1}=1}^{\infty} \sum_{k_{2}=1}^{\infty}\left|\lambda_{k_{1}+1, k_{2}+1}^{\theta}-\lambda_{k_{1}+1, k_{2}}^{\theta}-\lambda_{k_{1}, k_{2}+1}^{\theta}+\lambda_{k_{1}, k_{2}}^{\theta}\right| Y_{k_{1}, k_{2}}^{\theta}(f)_{p}
\end{aligned}
$$

converges, then there exists a function $f^{\left(\lambda, \beta_{1}, \beta_{2}\right)} \in L_{p}^{0}\left(\mathbb{T}^{2}\right)$, with the Fourier series $\sigma\left(f, \lambda, \beta_{1}, \beta_{2}\right)$, and

$$
\begin{aligned}
& Y_{2^{m_{1}-1,2^{m_{2}-1}}}\left(f^{\left(\lambda, \beta_{1}, \beta_{2}\right)}\right)_{p} \lesssim\left\{\lambda_{2^{m_{1}-1}, 2^{m_{2}-1}}^{\theta} Y_{2^{m_{1}-1,2^{m_{2}-1}}}^{\theta}(f)_{p}\right. \\
& +\sum_{\nu_{1}=m_{1}}^{\infty}\left|\lambda_{2^{\nu_{1}, 2^{m_{2}-1}}}^{\theta}-\lambda_{2^{\nu_{1}-1}, 2^{m_{2}-1}}^{\theta}\right| Y_{2^{\nu_{1}-1,2^{m_{2}-1}}}^{\theta}(f)_{p} \\
& +\sum_{\nu_{2}=m_{2}}^{\infty}\left|\lambda_{2^{m_{1}-1}, 2^{\nu_{2}}}^{\theta}-\lambda_{2^{m_{1}-1}, 2^{\nu_{2}-1}}^{\theta}\right| Y_{2^{m_{1}-1,2^{\nu_{2}-1}}}^{\theta}(f)_{p} \\
& +\sum_{\nu_{1}=m_{1}}^{\infty} \sum_{\nu_{2}=m_{2}}^{\infty} \mid \lambda_{2^{\nu_{1}}, 2^{\nu_{2}}}^{\theta}-\lambda_{2^{\nu_{1}-1}, 2^{\nu_{2}}}^{\theta} \\
& \left.-\lambda_{2^{\nu_{1}}, 2^{\nu_{2}-1}}^{\theta}+\lambda_{2^{\nu_{1}-1}, 2^{\nu_{2}-1}}^{\theta} \mid Y_{2^{\nu_{1}}-1,2^{\nu_{2}-1}}^{\theta}(f)_{p}\right\}^{\frac{1}{\theta}} .
\end{aligned}
$$

In subsection 3.4 we obtain the corresponding estimates from below. 


\section{Liouville-Weyl derivatives, best approximations, and moduli} of smoothness. $\left(L_{p}, L_{p}\right)$ inequalities

\subsection{Notation}

In this section we will give needed definitions and introduce some notation. Let $L_{p}=L_{p}[0,2 \pi](1 \leqslant p<\infty)$ be the space of $2 \pi$-periodic measurable functions that satisfy

$$
\|f\|_{p}=\left(\int_{0}^{2 \pi}|f(x)|^{p} d x\right)^{1 / p}<\infty .
$$

Let $L_{\infty} \equiv C[0,2 \pi]$ be the space of $2 \pi$-periodic continuous functions with

$$
\|f\|_{p}=\max _{x \in[0,1]}|f(x)|, \quad p=\infty .
$$

Let the series

$$
\frac{a_{0}(f)}{2}+\sum_{\nu=1}^{\infty}\left(a_{\nu}(f) \cos \nu x+b_{\nu}(f) \sin \nu x\right)
$$

be the Fourier series of $f \in L_{1}$. The Fourier coefficients of a function $f$ are given by the formulas

$$
\begin{aligned}
& a_{0}=\frac{1}{\pi} \int_{0}^{2 \pi} f(x) d x, \\
& a_{\nu}=\frac{1}{\pi} \int_{0}^{2 \pi} f(x) \cos \nu x d x, \quad \nu \in \mathbb{N}, \\
& b_{\nu}=\frac{1}{\pi} \int_{0}^{2 \pi} f(x) \sin \nu x d x, \quad \nu \in \mathbb{N} .
\end{aligned}
$$

Throughout the paper $n$ denotes an integer number. Let $S_{n}(f)$ denote the $n$-th partial sum of (1.1.1), $V_{n}(f)$ denote the de la Vallée-Poussin sum and $K_{n}(x)$ be the Fejér kernel, i.e.,

$$
\begin{gathered}
S_{n}(f)=\sum_{\nu=0}^{n} A_{\nu}(x)=\frac{a_{0}(f)}{2}+\sum_{\nu=1}^{n}\left(a_{\nu}(f) \cos \nu x+b_{\nu}(f) \sin \nu x\right) \\
V_{n}(f)=\frac{1}{n} \sum_{\nu=n}^{2 n-1} S_{\nu}(f), \quad V_{0}(f)=S_{0}(f)
\end{gathered}
$$




$$
K_{n}(x)=\frac{1}{n+1} \sum_{\nu=0}^{n}\left(\frac{1}{2}+\sum_{m=1}^{\nu} \cos m x\right) .
$$

The Fourier series of the $r$-th derivatives of $f$ is given as follow

$$
f^{(r)}(x) \sim \sum_{\nu=1}^{\infty} \nu^{r}\left(a_{\nu}(f) \cos \left(\nu x+\frac{r \pi}{2}\right)+b_{\nu}(f) \sin \left(\nu x+\frac{r \pi}{2}\right)\right),
$$

where $r \in \mathbb{N}$.

We will use the following definition of fractional differentiation, which was introduced by Weyl, see [52]. Let $f$ be a $2 \pi$-periodic integrable function and $\alpha>0$. Then the function $f^{(\alpha)}$ satisfying

$$
f^{(\alpha)}(x) \sim \sum_{\nu=1}^{\infty} \nu^{\alpha}\left(a_{\nu}(f) \cos \left(\nu x+\frac{\alpha \pi}{2}\right)+b_{\nu}(f) \sin \left(\nu x+\frac{\alpha \pi}{2}\right)\right)
$$

is called the Weyl fractional derivatives of order $\alpha$.

Recall that the conjugate series to (1.1.1) is given by

$$
\sum_{\nu=1}^{\infty}\left(a_{\nu} \sin \nu x-b_{\nu} \cos \nu x\right)
$$

The transformed Fourier series of (1.1.1) is defined by

$$
\sigma(f, \lambda, \beta):=\sum_{\nu=1}^{\infty} \lambda_{\nu}\left[a_{\nu} \cos \left(\nu x+\frac{\pi \beta}{2}\right)+b_{\nu} \sin \left(\nu x+\frac{\pi \beta}{2}\right)\right],
$$

where $\beta \in \mathbb{R}$ and $\lambda=\left\{\lambda_{n}\right\}$ is a sequence of positive numbers.

We call the function $\varphi(x) \sim \sigma(f, \lambda, \beta)$ the Liouville-Weyl derivative (or $(\lambda, \beta)$-derivative of the function $f)$ and denote it by $f^{(\lambda, \beta)}$. As an important example, for $\lambda_{n}=n^{r}, r>0, \beta=r$, we have $f^{(\alpha, \beta)}=f^{(r)}$ and for $\lambda_{n}=n^{r}, r>$ $0, \beta=r+1$ we have $f^{(\alpha, \beta)}= \pm \tilde{f}^{(r)}$, where $f^{(r)}$ is the fractional derivative in the sense of Weyl (see (1.1.3)) and $\tilde{f}^{(r)}$ is the $r$-fractional derivative of the conjugate function $\tilde{f}$.

Let $E_{n}(f)_{p}$ be the best approximation of a function $f \in L_{p}$ by trigonometric polynomials of degree at most $n$, i.e.,

$$
E_{n}(f)_{p}=\inf _{\alpha_{k}, \beta_{k} \in \mathbb{R}}\left\|f(x)-\sum_{k=0}^{n}\left(\alpha_{k} \cos k x+\beta_{k} \sin k x\right)\right\|_{p} .
$$


Throughout the work we use the notation $F \lesssim G$, with $F, G \geqslant 0$, for the estimate $F \leqslant C G$, where $C$ is a positive constant, independent of essential quantities in $F$ and $G$. Moreover $F \asymp G$ means that $F \lesssim G \lesssim F$ (in this case we say that $F$ is equivalent to $G$ ). Moreover, $C$ denotes positive constants not depending on essential parameters which may be different in different formulas.

\subsection{Moduli of smoothness}

First, we give the definition of the modulus of smoothness $\omega_{\alpha}(f, \delta)_{p}$ of order $\alpha, \alpha \in \mathbb{N}$. We define

$$
\omega_{\alpha}(f, \delta)_{p}=\sup _{|h| \leqslant \delta}\left\|\Delta_{h}^{\alpha} f\right\|_{p}
$$

where $\Delta_{h} f(x)=f(x+h)-f(x), \quad \Delta_{h}^{\alpha}=\Delta_{h} \Delta_{h}^{\alpha-1}$.

Let us now define the difference of fractional order $\alpha>0$ of function $f \in L_{p}$ at the point $x$ with increment $h$ by

$$
\Delta_{h}^{\alpha}(f)=\sum_{\nu=0}^{\infty}(-1)^{\nu}\left(\begin{array}{l}
\alpha \\
\nu
\end{array}\right) f(x+(\alpha-\nu) h),
$$

where $\left(\begin{array}{l}\alpha \\ \nu\end{array}\right)=1$ for $\nu=0,\left(\begin{array}{l}\alpha \\ \nu\end{array}\right)=\alpha$ for $\nu=1$, and

$$
\left(\begin{array}{l}
\alpha \\
\nu
\end{array}\right)=\frac{\alpha(\alpha-1) \cdots(\alpha-\nu+1)}{\nu !} \quad \text { for } \nu \geqslant 2 .
$$

Since $\left|\left(\begin{array}{l}\alpha \\ \nu\end{array}\right)\right| \leqslant C(\alpha) \nu^{-\alpha-1}, \nu \in \mathbb{N}$, then the following series

$$
C(\alpha):=\sum_{\nu=0}^{\infty}\left|\left(\begin{array}{l}
\alpha \\
\nu
\end{array}\right)\right|
$$

converges for all $\alpha>0$. Therefore, $\Delta_{h}^{\alpha} f \in L_{p}$ for any $f \in L_{p}, 1 \leqslant p \leqslant \infty$.

The main properties of fractional moduli of smoothness are similar to those of the classical moduli. First, we recall some basic properties of fractional differences.

Lemma 1.2.1. [7, 52, 66] Let $f, f_{1}, f_{2} \in L_{p}, 1 \leqslant p \leqslant \infty$, and $\alpha, \beta>0$. Then (a) $\triangle_{h}^{\alpha}\left(f_{1}+f_{2}\right)=\triangle_{h}^{\alpha} f_{1}+\triangle_{h}^{\alpha} f_{2}$; 
(b) $\triangle_{h}^{\alpha}\left(\triangle_{h}^{\beta} f\right)=\triangle_{h}^{\alpha+\beta} f$;

(c) $\left\|\triangle_{h}^{\alpha} f\right\|_{p} \lesssim\|f\|_{p}$.

Denote by $\omega_{\alpha}(f, \delta)_{p}$ the moduli of smoothness of fractional order $\alpha, \alpha>$ 0 , of the function $f \in L_{p}$, i.e.,

$$
\omega_{\alpha}(f, \delta)_{p}=\sup _{|h| \leqslant \delta}\left\|\Delta_{h}^{\alpha}(f)\right\|_{p},
$$

see $[7,52,66]$. Note that if $\alpha \in \mathbb{N}$ this definition coincides with (1.2.5).

The following properties of moduli of smoothness are well known.

Lemma 1.2.2. [7, 49, 72] Let $f, f_{1}, f_{2} \in L_{p}, 1<p<\infty, \alpha>0$. Then

(a) $\omega_{\alpha}(f, \delta)_{p}$ is nondecreasing nonnegative function of $\delta$, defined on $(0, \infty)$, with $\lim _{\delta \rightarrow 0+} \omega_{\alpha}(f, \delta)_{p}=0$;

(b) $\omega_{\alpha}\left(f_{1}+f_{2}, \delta\right)_{p} \leqslant \omega_{\alpha}\left(f_{1}, \delta\right)_{p}+\omega_{\alpha}\left(f_{2}, \delta\right)_{p}$;

(c) If $0<\delta_{2} \leqslant \delta_{1} \leqslant \pi$, then

$$
\frac{\omega_{\alpha}\left(f, \delta_{1}\right)_{p}}{\delta_{1}^{\alpha}} \lesssim \frac{\omega_{\alpha}\left(f, \delta_{2}\right)_{p}}{\delta_{2}^{\alpha}}
$$

(d) If $\lambda>1$, then

$$
\omega_{\alpha}(f, \lambda \delta)_{p} \lesssim \lambda^{\alpha} \omega_{\alpha}(f, \delta)_{p} .
$$

For any $p \geqslant 1$ and $\alpha>0$, introduce the periodic Sobolev space by

$$
W_{p}^{\alpha}:=\left\{g \in L_{p}: g^{(\alpha)} \in L_{p}, \quad g^{(\alpha)} \sim \sigma\left(g,\left\{\lambda_{n}=n^{\alpha}\right\}, \alpha\right)\right\},
$$

see (1.1.4). It is known $[7,34]$ that the modulus of smoothness is equivalent to the corresponding K-functional, that is,

$$
\omega_{\alpha}\left(f, \frac{1}{2^{n}}\right)_{p} \asymp K\left(f, \frac{1}{2^{n}}, L_{p}, W_{p}^{\alpha}\right), \quad \alpha>0,
$$

where $1 \leqslant p \leqslant \infty$ and

$$
K\left(f, \frac{1}{2^{n}}, L_{p}, W_{p}^{\alpha}\right):=\inf _{g \in W_{p}^{\alpha}}\left(\|f-g\|_{p}+2^{-\alpha n}\left\|g^{(\alpha)}\right\|_{p}\right) .
$$

The following lemma will play an important role in the proofs of our main results. 
Lemma 1.2.3. [55] Let $\alpha>0$. If $\varphi \in L_{p}, 1<p<\infty$, then

$$
\omega_{\alpha}\left(\varphi, \frac{1}{n}\right)_{p} \lesssim\left\|\varphi-S_{n}(\varphi)\right\|_{p}+n^{-\alpha}\left\|S_{n}^{(\alpha)}(\varphi)\right\|_{p} \lesssim \omega_{\alpha}\left(\varphi, \frac{1}{n}\right)_{p} .
$$

If $\varphi \in L_{p}, \quad 1 \leqslant p \leqslant \infty$, then

$$
\omega_{\alpha}\left(\varphi, \frac{1}{n}\right)_{p} \lesssim\left\|\varphi-V_{n}(\varphi)\right\|_{p}+n^{-\alpha}\left\|V_{n}^{(\alpha)}(\varphi)\right\|_{p} \lesssim \omega_{\alpha}\left(\varphi, \frac{1}{n}\right)_{p} .
$$

We mention that (1.2.8) and (1.2.9) are realization results for a modulus of smoothness of fractional order. Note that for $\alpha \in \mathbb{N}$ these results follow from [13]. To prove realization results, one needs the following lemma.

Lemma 1.2.4. [7, 66] Let $T_{n}(x)=\sum_{k=1}^{n}\left(a_{k} \cos k x+b_{k} \sin k x\right)$ be a trigonometric polynomial of degree $n, 1 \leqslant p \leqslant \infty$, and $\alpha>0$. Then

(a) $\left\|T_{n}^{(\alpha)}\right\|_{p} \lesssim n^{\alpha}\left\|\triangle_{\pi / n}^{\alpha} T_{n}\right\|_{p} ;$

(b) for all $h$ such that $0<|h| \leqslant \pi / n$, there holds $\left\|\triangle_{h}^{\alpha} T_{n}\right\|_{p} \lesssim n^{-\alpha}\left\|T_{n}^{(\alpha)}\right\|_{p}$.

\subsection{History of the question}

\section{Direct and inverse inequalities in approximation theory}

One of the main problems of the constructive theory of functions is to find a relationship between differential properties of functions and their structural or constructive characteristics. Direct and inverse approximation theorems answer this question. Direct theorems for $L_{p}, 1 \leqslant p \leqslant \infty$, deal with estimates of the best approximation in terms of moduli of smoothness:

$$
\begin{gathered}
E_{n}(f)_{p} \leqslant C(p, \alpha) \omega_{\alpha}\left(f, \frac{1}{n}\right)_{p}, n, \alpha \in \mathbb{N}, \\
E_{n}(f)_{p} \leqslant \frac{C(p, \alpha, r)}{n^{r}} \omega_{\alpha}\left(f^{(r)}, \frac{1}{n}\right)_{p} \quad n, \alpha, r \in \mathbb{N} .
\end{gathered}
$$

Inequality (1.3.10) was obtained by Jackson [27] for $\alpha=1, p=\infty$. Moreover, Akhiezer [1] proved it for $\alpha=2,1 \leqslant p \leqslant \infty$. In the case $\alpha \geqslant 3$ and $p=\infty$, (1.3.10) was proved by Stechkin [59]. For $\alpha \geqslant 3$ and $1 \leqslant p<\infty$ see [8], [78]. The second inequality was obtained in [78], also see [8], [67], [87]. 
Inverse theorems for $L_{p}, 1 \leqslant p \leqslant \infty$, are written as follows: for $n, \alpha, r \in \mathbb{N}$, we have

$$
\begin{gathered}
\omega_{\alpha}\left(f, \frac{1}{n}\right)_{p} \leqslant \frac{C(p, \alpha)}{n^{\alpha}} \sum_{\nu=0}^{n}(\nu+1)^{\alpha-1} E_{\nu}(f)_{p}, \\
\omega_{\alpha}\left(f^{(r)}, \frac{1}{n}\right)_{p} \leqslant C(p, r, \alpha)\left(\frac{1}{n^{\alpha}} \sum_{\nu=0}^{n}(\nu+1)^{\alpha+r-1} E_{\nu}(f)_{p}+\sum_{\nu=n+1}^{\infty} \nu^{r-1} E_{\nu}(f)_{p}\right) .
\end{gathered}
$$

Inequality (1.3.12) was proved by Salem [51] for $\alpha=1, p=\infty$; for $\alpha \in \mathbb{N}$ see [8], [78]. Inequality (1.3.13) was obtained by Stechkin [59] for $p=\infty$, for other cases see [8], [78]. Later on, inequalities (1.3.10) - (1.3.13) were extended by Timan [79], [80], [81]. Note that direct and inverse inequalities (1.3.10)-(1.3.13) influenced substantially the further research [8], [78].

Let us now discuss several well-known inequalities related to direct and inverse theorems. Further, we present the most important inequalities for norms, best approximations, and moduli of smoothness of the $r$-th derivative in terms of those of the function $f$ itself.

The following result was proved by Bernstein [4] for $p=\infty$ (for $1 \leqslant$ $p<\infty$, see [8]):

If $f \in L_{p}, 1 \leqslant p \leqslant \infty$, and $\sum_{k=0}^{\infty}(k+1)^{r-1} E_{k}(f)_{p}<\infty, r \in \mathbb{N}$, then

$$
\left\|f^{(r)}\right\|_{p} \leqslant C(r) \sum_{k=0}^{\infty}(k+1)^{r-1} E_{k}(f)_{p}
$$

Marcinkiewicz [42] and Besov [5] obtained an improvement of this inequality for $1<p<\infty$ :

$$
\left\|f^{(r)}\right\|_{p} \leqslant C(r, p)\left(\sum_{k=0}^{\infty}(k+1)^{\theta r-1} E_{k}^{\theta}(f)_{p}\right)^{\frac{1}{\theta}}, \quad \theta=\min (2, p) .
$$

Later on, Stechkin [60] for $p=\infty$ and Konyushkov [37] for $1<p<\infty$ obtained the following inequality for the best approximations of $f^{(r)}$ :

$$
E_{n}\left(f^{(r)}\right)_{p} \leqslant C(r, p)\left(n^{r} E_{n}(f)_{p}+\sum_{k=n+1}^{\infty} k^{r-1} E_{k}(f)_{p}\right), \quad r, n \in \mathbb{N} .
$$


Inequality (1.3.15) was extended by Timan [82] for the case $1<p<\infty$ as follows

$$
E_{n}\left(f^{(r)}\right)_{p} \leqslant C(r, p)\left(n^{r} E_{n}(f)_{p}+\left(\sum_{k=n+1}^{\infty} k^{\theta r-1} E_{k}^{\theta}(f)_{p}\right)^{\frac{1}{\theta}}\right)
$$

where $\theta=\min (2, p), r, n \in \mathbb{N}$.

Moreover, Stechkin [60] proved the similar result for $\tilde{f}^{(r)}$ :

$$
E_{n}\left(\tilde{f}^{(r)}\right)_{p} \leqslant C(r)\left(n^{r} E_{n}(f)_{p}+\sum_{k=n+1}^{\infty} k^{r-1} E_{k}(f)_{p}\right), \quad r, n \in \mathbb{N},
$$

where $p=1$ or $p=\infty$.

Corresponding estimates for moduli of smoothness was obtained by Johnen and Scherer [29] (see also [8, pp.178-179]):

$$
\omega_{k}\left(f^{(r)}, \frac{1}{n}\right)_{p} \leqslant C(k, r, p) \sum_{\nu=n+1}^{\infty} \nu^{r-1} \omega_{k+r}\left(f, \frac{1}{\nu}\right)_{p}, \quad 1 \leqslant p \leqslant \infty, r, k, n \in \mathbb{N} .
$$

From the result by Bari and Stechkin [61] one has

$$
\omega_{k}\left(\tilde{f}^{(r)}, \frac{1}{n}\right)_{p} \leqslant C(k, r)\left(\frac{1}{n^{k}} \sum_{\nu=1}^{n} \nu^{k+r-1} E_{\nu}(f)_{p}+\sum_{\nu=n+1}^{\infty} \nu^{r-1} E_{\nu}(f)_{p}\right),
$$

where $p=1, \infty$ and $r, k, n \in \mathbb{N}$.

Moreover, in light of Jackson's inequality, we have the following estimate:

$\omega_{k}\left(\tilde{f}^{(r)}, \frac{1}{n}\right)_{p} \leqslant C(k, r)\left(\frac{1}{n^{k}} \sum_{\nu=1}^{n} \nu^{k+r-1} \omega_{k+r}\left(f, \frac{1}{\nu}\right)_{p}+\sum_{\nu=n+1}^{\infty} \nu^{r-1} \omega_{k+r}\left(f, \frac{1}{\nu}\right)_{p}\right)$,

where $p=1, \infty$ and $r, k, n \in \mathbb{N}$.

Inequality (1.3.17) was extended by Ditzian and Tikhonov [11] for the case of $0<p<\infty$ as follows

$\omega_{k}\left(f^{(r)}, \frac{1}{n}\right) \leqslant C(k, r, p)\left(\sum_{\nu=n+1}^{\infty} \nu^{r \theta-1} \omega_{k+r}^{\theta}\left(f, \frac{1}{\nu}\right)_{p}\right)^{\frac{1}{\theta}}, \quad \theta=\min (2, p), r, k, n \in \mathbb{N}$, 
see also [55].

Let us now mention estimates of $E_{n}\left(f^{(r)}\right)_{p}$ from below. The following inequality was proved for $r \in \mathbb{N}$ in [8] and for $r>0$ in [54]:

$$
n^{r} E_{n}(f)_{p} \leqslant C(r) E_{n}\left(f^{(r)}\right)_{p}, \quad 1 \leqslant p \leqslant \infty .
$$

Simonov and Tikhonov [54,55] obtained estimates from below of the best approximations of $f^{(r)}$ :

$$
\left(n^{r \tau} E_{n}^{\tau}(f)_{p}+\sum_{k=n+1}^{\infty} k^{\tau r-1} E_{k}^{\tau}(f)_{p}\right)^{\frac{1}{\tau}} \leqslant C(r) E_{n}\left(f^{(r)}\right)_{p}
$$

where $1<p<\infty, \tau=\max (2, p)$. For the case $0<p<1$, see [35].

\section{The problem of multipliers for trigonometric polynomials}

Let us consider the following problem. Let $\mathbb{T}_{n}$ be the space of trigonometric polynomials of degree of most $n$, i.e.,

$$
t_{n}(x)=\frac{a_{0}}{2}+\sum_{k=1}^{n}\left(a_{k} \cos k x+b_{k} \sin k x\right) \in \mathbb{T}_{n}
$$

with the norm $\left\|t_{n}(x)\right\|_{\infty}=\max _{x}\left|t_{n}(x)\right|$. By $\Lambda=\left\{\lambda_{k}\right\}_{k=0}^{\infty}$ we define a multiplier sequence. We set

$$
\tau_{n}(x)=\tau_{n}\left(x, t_{n}\right)=\lambda_{0} \frac{a_{0}}{2}+\sum_{k=1}^{n} \lambda_{k}\left(a_{k} \cos k x+b_{k} \sin k x\right)
$$

and

$$
\widetilde{\tau}_{n}(x)=\widetilde{\tau}_{n}\left(x, t_{n}\right)=\sum_{k=1}^{n} \lambda_{k}\left(a_{k} \sin k x-b_{k} \cos k x\right),
$$

where $a_{k}$ and $b_{k}$ are the coefficients of the polynomial (1.3.20). Several authors investigated the behavior of the following two expressions:

$$
M_{n}=M_{n}(\Lambda)=\sup _{\left\|t_{n}\right\|_{\infty} \leqslant 1}\left\|\tau_{n}\left(t_{n}\right)\right\|_{\infty}
$$

and

$$
\widetilde{M}_{n}=\widetilde{M}_{n}(\Lambda)=\sup _{\left\|t_{n}\right\|_{\infty} \leqslant 1}\left\|\widetilde{\tau}_{n}\left(t_{n}\right)\right\|_{\infty}
$$

By definition, the numbers $M$ and $\widetilde{M}_{n}$ are the smallest constants for which inequalities

$$
\left\|\tau_{n}\left(t_{n}\right)\right\| \leqslant M_{n}\left\|t_{n}\right\|
$$


and

$$
\left\|\widetilde{\tau}_{n}\left(t_{n}\right)\right\| \leqslant \widetilde{M}_{n}\left\|t_{n}\right\|
$$

are valid for any polynomial $t_{n} \in \mathbb{T}_{n}$. The problem of estimating the constants $M_{n}$ and $\widetilde{M}_{n}$ is called the problem of multipliers for trigonometric polynomials. One of the first result related to the problem of multipliers for trigonometric polynomials was the result Bernstein for $\lambda=k([4])$. Szego [65] and Fejer [18] proved that $M_{n}=\widetilde{M}_{n}=\lambda_{n}$ in the case $\lambda_{0}=0, \Delta \lambda_{k}=\lambda_{k}-\lambda_{k-1} \geqslant$ $0(k=1,2, \ldots, n)$, and $\Delta^{2} \lambda_{k}=\lambda_{k}-2 \lambda_{k-1}+\lambda_{k-2} \geqslant 0(k=2, \ldots, n)$.

Later on, Sokolov [58] obtained the following estimate

$$
M_{n} \leqslant \frac{1}{n}\left(\lambda_{n}+2 \sum_{k=1}^{n-1} \lambda_{k}\right),
$$

where $\lambda_{n}$ satisfies the following conditions:

$$
\begin{array}{r}
\lambda_{0}=0, \Delta \lambda_{k} \geqslant 0(k=1,2, \ldots, n), \\
\Delta^{2} \lambda_{k} \leqslant 0(k=2, \ldots, n) .
\end{array}
$$

In the case when conditions (1.3.21) hold, the next result was proved by Stechkin [61] in 1950:

$$
\widetilde{M}_{n}(\Lambda) \sim P_{n}=\sum_{k=1}^{n} \frac{\lambda_{k}}{k} .
$$

We will extend the above mentioned results in Lemma 1.4.5 below.

Our next problem is closely related to embeddings of function spaces, see [23]-[26], [45]-[47],[74], [75], and [84].

\section{Inequalities for best approximation and moduli of smoothness} of Liouville - Weyl derivatives

In [54] and [55], the authors considered the generalized derivatives in the sense of Liouville-Weyl and extended mentioned above results as follows. Recall that $\sigma(f, \lambda, \beta)$ is defined by (1.1.4).

Theorem A1. Let $1<p<\infty, \theta=\min (2, p), \quad \beta \in \mathbb{R}$, and $\lambda=\left\{\lambda_{n}\right\}_{n=1}^{\infty}$ be non-decreasing sequence of positive numbers satisfying $\Delta_{2}$-condition, i.e., $\lambda_{2 n} \leqslant \lambda_{n}$. If $f \in L_{p}$ and

$$
\sum_{n=1}^{\infty}\left(\lambda_{n+1}^{\theta}-\lambda_{n}^{\theta}\right) E_{n}^{\theta}(f)_{p}<\infty
$$


then there exists a function $f^{(\lambda, \beta)} \in L_{p}$ with the Fourier series $\sigma(f, \lambda, \beta)$ and

$$
\begin{aligned}
\left\|f^{(\lambda, \beta)}\right\|_{p} & \lesssim\left\{\lambda_{1}^{\theta} E_{0}^{\theta}(f)_{p}+\sum_{n=1}^{\infty}\left(\lambda_{n+1}^{\theta}-\lambda_{n}^{\theta}\right) E_{n}^{\theta}(f)_{p}\right\}^{\frac{1}{\theta}}, \\
E_{n}\left(f^{(\lambda, \beta)}\right) & \lesssim\left\{\lambda_{n}^{\theta} E_{n}^{\theta}(f)_{p}+\sum_{k=n+1}^{\infty}\left(\lambda_{k+1}^{\theta}-\lambda_{k}^{\theta}\right) E_{k}^{\theta}(f)_{p}\right\}^{\frac{1}{\theta}} .
\end{aligned}
$$

Moreover, if $\alpha>0$ and $\left\{\frac{\lambda_{n}}{n^{r}}\right\}$ is decreasing for some $r>0$, then

$$
\begin{aligned}
\omega_{\alpha}\left(f^{(\lambda, \beta)}, \frac{1}{n}\right)_{p} \lesssim\left\{n^{-\alpha \theta} \sum_{k=1}^{n}\left(\frac{\lambda_{k}^{\theta}}{k^{r \theta}}-\frac{\lambda_{k+1}^{\theta}}{(k+1)^{r \theta}}\right) k^{(r+\alpha) \theta} \omega_{\alpha+r}^{\theta}\left(f, \frac{1}{k}\right)_{p}\right. \\
\left.+\sum_{k=n+2}^{\infty}\left(\lambda_{k+1}^{\theta}-\lambda_{k}^{\theta}\right) \omega_{\alpha+r}^{\theta}\left(f, \frac{1}{k}\right)_{p}+\lambda_{n+1}^{\theta} \omega_{\alpha+r}^{\theta}\left(f, \frac{1}{n+1}\right)_{p}\right\}^{\frac{1}{\theta}} .
\end{aligned}
$$

In the limiting cases $p=1$ or $p=\infty$ one has to assume additional conditions on the sequence $\left\{\lambda_{n}\right\}$. The following theorem holds $[54,55]$.

Theorem A2. Let $p=1, \infty, \beta \in \mathbb{R}$ and $\lambda=\left\{\lambda_{n}\right\}_{n=1}^{\infty}$ be non-decreasing sequence of positive numbers satisfying $\Delta_{2}-$ condition and such that $\Delta \lambda_{n} \leqslant$ $C \Delta \lambda_{2 n}$ and $\Delta^{2} \lambda_{n} \geqslant 0$ (or $\leqslant 0$ ).

If $f \in L_{p}$ and

$$
\left|\cos \frac{\pi \beta}{2}\right| \sum_{n=1}^{\infty}\left(\lambda_{n+1}-\lambda_{n}\right) E_{n}(f)_{p}+\left|\sin \frac{\pi \beta}{2}\right| \sum_{n=1}^{\infty} \lambda_{n} \frac{E_{n}(f)_{p}}{n}<\infty,
$$

then there exists a function $f^{(\lambda, \beta)} \in L_{p}$ with the Fourier series $\sigma(f, \lambda, \beta)$ and

$$
\begin{aligned}
\left\|f^{(\lambda, \beta)}\right\|_{p} & \lesssim\left|\cos \frac{\pi \beta}{2}\right| \sum_{n=1}^{\infty}\left(\lambda_{n+1}-\lambda_{n}\right) E_{n}(f)_{p}+\left|\sin \frac{\pi \beta}{2}\right| \sum_{n=1}^{\infty} \lambda_{n} \frac{E_{n}(f)_{p}}{n} \\
E_{n}\left(f^{(\lambda, \beta)}\right)_{p} & \lesssim \lambda_{n} E_{n}(f)_{p}+\left|\cos \frac{\pi \beta}{2}\right| \sum_{\nu=n+1}^{\infty}\left(\lambda_{\nu+1}-\lambda_{\nu}\right) E_{\nu}(f)_{p} \\
+ & \sin \frac{\pi \beta}{2} \mid \sum_{\nu=n+1}^{\infty} \lambda_{\nu} \frac{E_{\nu}(f)_{p}}{\nu}
\end{aligned}
$$


Moreover, if $\alpha>0$ and the sequence $\left\{\lambda_{n}\right\}_{n=1}^{\infty}$ is such that $\left\{\frac{\lambda_{n}}{n^{\rho}}\right\}$ is decreasing for some $\rho>0$ and, for some $\tau>0$, there holds $\Delta^{2}\left(\frac{\lambda_{n}}{n^{r}}\right) \geqslant 0$ with $r=$ $\rho+\tau \operatorname{sign}\left|\sin \frac{(\beta-\rho) \pi}{2}\right|$, then

$$
\begin{aligned}
\omega_{\alpha}\left(f^{(\lambda, \beta)}, \frac{1}{n}\right) & \lesssim\left|\cos \frac{\pi \beta}{2}\right| \sum_{\nu=n+2}^{\infty}\left(\lambda_{\nu+1}-\lambda_{\nu}\right) \omega_{\alpha+r}\left(f, \frac{1}{\nu}\right)_{p} \\
& +\left|\sin \frac{\pi \beta}{2}\right| \sum_{\nu=n+2}^{\infty} \frac{\lambda_{\nu}}{\nu} \omega_{\alpha+r}\left(f, \frac{1}{\nu}\right)_{p}+\lambda_{n+1} \omega_{\alpha+r}\left(f, \frac{1}{n+1}\right)_{p} \\
& +n^{-\alpha} \sum_{\nu=1}^{n}\left(\frac{\lambda_{\nu}}{\nu^{\rho}}-\frac{\lambda_{\nu+1}}{(\nu+1)^{\rho}}\right) \nu^{\alpha+\rho} \omega_{\alpha+\rho}\left(f, \frac{1}{\nu}\right)_{p} .
\end{aligned}
$$

In particular, (1.3.22) and (1.3.25) generalize (1.3.14), while (1.3.23) and (1.3.26) generalize (1.3.15) and (1.3.16). In addition, (1.3.17), (1.3.18), and (1.3.19) follow from (1.3.24) and (1.3.27).

Our main goal in this section (see subsection 1.6 and 1.7) is to prove analogues of Theorems A1 and A2 by considering a more general class of sequences $\left\{\lambda_{n}\right\}$. We replace the monotonicity condition on $\left\{\lambda_{n}\right\}$ and $\left\{\Delta \lambda_{n}\right\}$ by general monotonicity.

\subsection{General monotone sequences and their properties}

In this subsection, we present some definition of monotone type sequences. In particular, we will give the definition of general monotone sequences introduced in [69] and their basic properties. First, we recall the definition of monotone sequences:

$$
M=\left\{\lambda=\left\{\lambda_{n}\right\}_{n \in \mathbb{N}}: \lambda_{n} \in \mathbb{R}, \lambda_{n}>0, \quad \lambda_{n} \downarrow 0 \text {, i.e., } \lambda_{n} \geqslant \lambda_{n+1} \geqslant \cdots \rightarrow 0\right\} .
$$

The concept of quasi-monotone sequence was introduced in $[57,63]$ as follows:

$$
Q M=\left\{\lambda=\left\{\lambda_{n}\right\}_{n \in \mathbb{N}}: \lambda_{n} \in \mathbb{R}, \exists \tau \geqslant 0 \text { such that } \frac{\lambda_{n}}{n^{\tau}} \downarrow\right\} .
$$

Now we give the definition of a more general class of O-regularly varying 
quasi-monotone sequences (see [62]):

$$
\begin{array}{r}
O R V Q M=\left\{\lambda=\left\{\lambda_{n}\right\}_{n \in \mathbb{N}}: \lambda_{n} \in \mathbb{R}, \exists \mu_{n} \geqslant 0, \mu_{n} \nearrow, \mu_{2 n} \leqslant C \mu_{n}\right. \\
\text { such that } \left.\frac{\lambda_{n}}{\mu_{n}} \downarrow\right\} .
\end{array}
$$

Leindler in [39] defined another class of sequences named as sequences of rest bounded variation (denoted by $R B V S$ ), keeping some properties of decreasing sequences:

$R B V S=\left\{\lambda=\left\{\lambda_{n}\right\}_{n \in \mathbb{N}}: \lambda_{n} \in \mathbb{R} \quad \lambda_{n} \rightarrow 0, \sum_{\nu=n}^{\infty}\left|\lambda_{\nu+1}-\lambda_{\nu}\right| \leqslant C\left|\lambda_{n}\right| \forall n \in \mathbb{N}\right\}$.

In particular, $\left\{\lambda_{n}\right\} \in R B V S$ implies that $\left|\lambda_{k}\right| \leqslant C\left|\lambda_{n}\right|$ for any $n \leqslant k$.

The classes $Q M$ (or $O R V Q M$ ) and $R B V S$ are not comparable (see [40], [69]). It is clear that $M \subset O R V Q M \cap R B V S$.

Recently, Tikhonov [69, 70] introduced the following new class of sequences, which contains all classes of sequences mentioned earlier.

Definition 1.4.1. A sequence $\lambda:=\left\{\lambda_{n}\right\}_{n=1}^{\infty}$ of real numbers is said to be general monotone, written $\lambda \in G M$, if the relation

$$
\sum_{k=n}^{2 n}\left|\lambda_{k}-\lambda_{k+1}\right| \leqslant C\left|\lambda_{n}\right|
$$

holds for all integer $n$, where the constant $C$ is independent of $n$.

In what follows we will consider only non-negative $G M$-sequences. The class $G M$ contains monotone or quasi-monotone sequences. Moreover, in [69], the author showed that $O R V Q M \cup R B V S \subsetneq G M$. We summarize various generalizations of the monotone conditions in the following Figure 1.

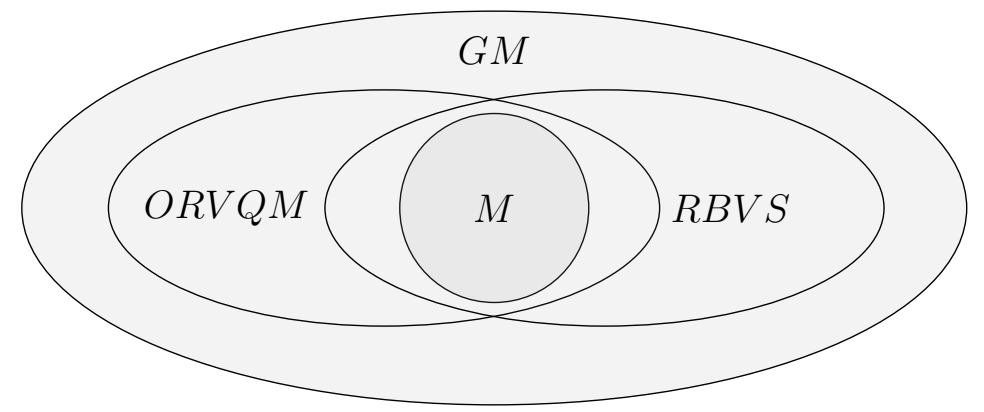

Figure 1: Relationships between different generalized classes of monotonic sequences. 
The following characterization of general monotone sequences is known. Lemma 1.4.1. [69] $\left\{\lambda_{n}\right\} \in G M$ if and only if there exists $C>0$ such that

1. $\left|\lambda_{k}\right| \leqslant C\left|\lambda_{n}\right|$ for $n \leqslant k \leqslant 2 n$,

2. $\sum_{k=n}^{N}\left|\Delta \lambda_{k}\right| \leqslant C\left(\left|\lambda_{n}\right|+\sum_{k=n+1}^{N} \frac{\left|\lambda_{k}\right|}{k}\right) \quad$ for any $\quad n<N$.

The following multiplier property holds true.

Lemma 1.4.2. [41] If $\lambda=\left\{\lambda_{n}\right\} \in G M$ and $\eta=\left\{\eta_{n}\right\} \in G M$ then $\lambda \eta=$ $\left\{\lambda_{n} \eta_{n}\right\} \in G M$.

Note that any $G M$-sequence satisfies $\Delta_{2}$-condition, that is, $\lambda_{2 n} \leqslant C \lambda_{n}$, see Lemma 1.4.1.

Clearly any monotone sequence belongs to the $G M$ class but the reverse is not always true. Some examples one can find in [41] and [69]. Below we give examples of $\left\{\lambda_{n}\right\}$ such that $\left\{\Delta \lambda_{n}\right\} \in G M$ and $\left\{\Delta \frac{\lambda_{n}}{n^{r}}\right\} \in G M$ but $\left\{\lambda_{n}\right\}$ is not monotone or convex. These examples are related to Theorems A1 and A2 and Theorems 1.6.1 and 1.7.1 below.

Example 1.4.1. Let us define

$$
\lambda_{n}= \begin{cases}n^{\alpha+1}, & 2^{k}<n<2^{k+1} \\ n^{\alpha+1}+c, & n=2^{k}\end{cases}
$$

where $\alpha>1$ and $c>2^{k(\alpha+1)}$. It is easy to check that

(1). $\left\{\lambda_{n}\right\} \in G M$

(2). $\left\{\Delta \lambda_{n}\right\} \in G M$,

(3). $\left\{\frac{\lambda_{n}}{n^{r}}\right\} \in G M$, where $r>0$,

(4). $\left\{\Delta \frac{\lambda_{n}}{n^{r}}\right\} \in G M$, where $r>0$.

Example 1.4.2. Let $\left\{\lambda_{n}\right\}$ be non-decreasing sequence satisfying the condition $\lambda_{2 n} \leqslant C \lambda_{n}$. We define

$$
\left\{\lambda_{n}^{\prime}\right\}:=\left\{\begin{array}{l}
\lambda_{n}, \quad 2^{k} \leqslant n \leqslant 2^{k}+2^{k-1}-1 \\
\lambda_{2^{k}+2^{k-1}+n-1}=\lambda_{2^{k}+2^{k-1}-n}, \quad 1 \leqslant n \leqslant 2^{k-1}, k \in \mathbb{N} .
\end{array}\right.
$$


It is easy to see that $\left\{\lambda_{n}^{\prime}\right\} \in G M$.

Remark 1.4.1. One can also define another class of general monotone sequences (see [69]):

$$
\sum_{k=n}^{2 n}\left|\lambda_{k}-\lambda_{k+1}\right| \leqslant C\left|\lambda_{2 n}\right|,
$$

where the constant $C$ is independent of $n$. We denote the class of such sequences by $G M^{\uparrow}$.

The following lemma provides a characterization of sequences from $G M^{\uparrow}$. The proof follows the same line as the proof of Lemma 1.4.1 (see [69]).

Lemma 1.4.3. A sequences $\left\{\lambda_{n}\right\} \in G M^{\uparrow}$ if and only if there exists $C>0$ such that

1. $\left|\lambda_{n}\right| \leqslant C\left|\lambda_{k}\right|$ for $n \leqslant k \leqslant 2 n$,

2. $\sum_{k=n}^{N}\left|\Delta \lambda_{k}\right| \leqslant C\left(\left|\lambda_{N}\right|+\sum_{k=n+1}^{N} \frac{\left|\lambda_{k}\right|}{k}\right) \quad$ for any $n<N$.

Note that any non-decreasing sequence belongs to $G M^{\uparrow}$.

To treat the case $p=1$ or $p=\infty$ in subsection 1.7 , we will need the following two lemmas.

Lemma 1.4.4. If $\left\{\Delta \lambda_{n}\right\} \in G M$, then

$$
\begin{aligned}
& \sum_{m=2^{n}}^{2^{n+2}-2}\left|\Delta^{2} \lambda_{m+2}\right|(m+1)+\left(2^{n+2}-1\right)\left|\Delta \lambda_{2^{n+2}-1}\right| \\
& +\left(2^{n+1}+2\right)\left|\Delta \lambda_{2^{n}}\right| \leqslant C \sum_{m=2^{n-1}}^{2^{n}}\left|\Delta \lambda_{m}\right| .
\end{aligned}
$$

Proof. Using the property of $G M$ sequences, we have the following inequality

$$
\begin{aligned}
& \sum_{m=2^{n}}^{2^{n+2}-2}\left|\Delta^{2} \lambda_{m+2}\right|(m+1)+\left(2^{n+2}-1\right)\left|\Delta \lambda_{2^{n+2}-1}\right|+\left(2^{n+1}+2\right)\left|\Delta \lambda_{2^{n}}\right| \\
& \leqslant C\left(\left(2^{n+2}-1\right)\left|\Delta \lambda_{2^{n}+2}\right|+\left(2^{n+2}-1\right)\left|\Delta \lambda_{2^{n}}\right|+\left(2^{n+1}+2\right)\left|\Delta \lambda_{2^{n}}\right|\right) \\
& \leqslant C 2^{n}\left|\Delta \lambda_{2^{n}}\right| \leqslant C \sum_{m=2^{n-1}}^{2^{n}}\left|\Delta \lambda_{m}\right|,
\end{aligned}
$$


which proves the statement of the lemma.

For a given sequence $\left\{\lambda_{n}\right\}_{n=1}^{\infty}$ define $\Delta \lambda_{n}=\lambda_{n}-\lambda_{n+1}$ and $\Delta^{2} \lambda_{n}=$ $\Delta\left(\Delta \lambda_{n}\right)=\lambda_{n}-2 \lambda_{n+1}+\lambda_{n+2}$.

Lemma 1.4.5. Let $p=1$ or $p=\infty$ and $\left\{\Delta \lambda_{n}\right\} \in G M$. Let

$$
\begin{aligned}
& T_{n}(x)=\sum_{\nu=1}^{n}\left(a_{\nu} \cos \nu x+b_{\nu} \sin \nu x\right), \\
& T_{n}(\lambda, x)=\sum_{\nu=1}^{n} \lambda_{\nu}\left(a_{\nu} \cos \nu x+b_{\nu} \sin \nu x\right) .
\end{aligned}
$$

Then the following inequality holds

$$
\begin{aligned}
& \left\|T_{2^{n+2}}(\lambda, x)-T_{2^{n}}(\lambda, x)\right\|_{p} \\
& \leqslant C\left(\sum_{k=2^{n-1}}^{2^{n}}\left|\Delta \lambda_{k}\right|+\left|\lambda_{2^{n+2}}\right|\right)\left\|T_{2^{n+2}}(f)-T_{2^{n}}(f)\right\|_{p} .
\end{aligned}
$$

Proof. Applying the Abel transformation twice, we get

$$
\begin{aligned}
& \left\|T_{2^{n+2}}(\lambda, f)-T_{2^{n}}(\lambda, f)\right\|_{p} \\
& =\left\|\frac{1}{\pi} \int_{-\pi}^{\pi}\left(T_{2^{n+2}}(f)-T_{2^{n}}(f)\right)(x+u) \sum_{\nu=2^{n}}^{2^{n+2}}\left(\lambda_{\nu} \cos \nu u\right) d u\right\|_{p} \\
& \leqslant \| \frac{1}{\pi} \int_{-\pi}^{\pi}\left(T_{2^{n+2}}(f)-T_{2^{n}}(f)\right)(x+u)\left(\sum_{\nu=2^{n}}^{2^{n+2}-1}\left(\lambda_{\nu}-\lambda_{\nu+1}\right) \sum_{m=0}^{\nu} \cos m u\right. \\
& \left.+\lambda_{2^{n+2}} \sum_{m=0}^{2^{n+2}} \cos m u-\lambda_{2^{n}} \sum_{m=0}^{2^{n}-1} \cos m x\right) d u \|_{p} \\
& \leqslant \| \frac{1}{\pi} \int_{-\pi}^{\pi}\left(T_{2^{n+2}}(f)-T_{2^{n}}(f)\right)(x+u)\left(\sum_{\nu=2^{n}}^{2^{n+2}-2} \Delta^{2} \lambda_{\nu} \sum_{k=0}^{\nu} \sum_{m=0}^{k} \cos m u\right. \\
& +\Delta \lambda_{2^{n+2}-1} \sum_{k=0}^{2^{n+2}-1} \sum_{m=0}^{k} \cos m u-\Delta \lambda_{2^{n}} \sum_{k=0}^{2^{n}} \sum_{m=0}^{k} \cos m u \\
& \left.+\lambda_{2^{n+2}} \sum_{m=0}^{2^{n+2}-1} \cos m u-\lambda_{2^{n}} \sum_{m=0}^{2^{n}-1} \cos m u\right)_{p} d u \|_{p}=:
\end{aligned}
$$


Using the definition of the Fejér kernel, we have

$$
\begin{aligned}
I & =\| \frac{1}{\pi} \int_{-\pi}^{\pi}\left(T_{2^{n+2}}(f)-T_{2^{n}}(f)\right)(x+u)\left(\sum_{\nu=2^{n}}^{2^{n+2}-2} \Delta^{2} \lambda_{\nu} K_{\nu}(u)(\nu+1)\right. \\
& +\sum_{\nu=2^{n}}^{2^{n+2}-2} \Delta^{2} \lambda_{\nu} \frac{(\nu+1)}{2}+\Delta \lambda_{2^{n+2}-1} K_{2^{n+2}-1}(u) 2^{n+2}+\Delta \lambda_{2^{n+2}-1} 2^{n+1}- \\
& -\Delta \lambda_{2^{n}} K_{2^{n}}(u)\left(2^{n}+1\right)-\Delta \lambda_{2^{n}} \frac{2^{n}+1}{2} \\
& \left.+\lambda_{2^{n+2}} \sum_{m=0}^{2^{n+2}-1} \cos m u-\lambda_{2^{n}} \sum_{m=0}^{2^{n}-1} \cos m u\right) d u \|_{p} \\
& \leqslant\left\|T_{2^{n+2}}(f)-T_{2^{n}}(f)\right\|_{p}\left(2 \sum_{\nu=2^{n}}^{2^{n+2}-2}\left|\Delta^{2} \lambda_{\nu}\right|(\nu+1)\right. \\
& \left.+\left|\Delta \lambda_{2^{n+2}-1}\right| 2^{n+3}+2\left|\Delta \lambda_{2^{n}}\right|\left(2^{n}+1\right)\right) \\
& +\left\|\left|\lambda_{2^{n+2}}\right|\left(T_{2^{n+2}}(f)-T_{2^{n}}(f)\right)\right\|_{p} .
\end{aligned}
$$

By Lemma 1.4.4,

$$
I \leqslant C\left(\sum_{k=2^{n-1}}^{2^{n}}\left|\Delta \lambda_{k}\right|+\left|\lambda_{2^{n+2}}\right|\right)\left\|T_{2^{n+2}}(f)-T_{2^{n}}(f)\right\|_{p},
$$

completing the proof.

The following result provides a simple multiplier property of general monotone sequences.

Lemma 1.4.6. Let $\left\{a_{n}\right\} \in G M$. Then $\left\{n^{\alpha} a_{n}\right\} \in G M, \alpha \in \mathbb{R}$.

The proof follows from Lemma 1.4.2.

Lemma 1.4.7. Let $\lambda=\{\lambda\}_{m=1}^{\infty} \in G M$, then

$$
\left|\lambda_{2^{m}}\right| \lesssim 2^{m \rho} \Lambda_{2^{m}},
$$

where $\Lambda_{2^{m}}:=\left(\sum_{k=1}^{2^{m}} \frac{\left.\lambda_{k}\right|^{q}}{k^{q \rho+1}}\right)^{\frac{1}{q}}$ and $\rho, q>0$. 
Proof. Using properties of $G M$ sequences, we get

$$
\begin{aligned}
& 2^{m \rho}\left|\lambda_{2^{m}}\right|\left(2^{-m \rho q}\right)^{\frac{1}{q}} \asymp 2^{m \rho}\left|\lambda_{2^{m}}\right|\left(\sum_{k=2^{m-1}}^{2^{m}} \frac{1}{k^{q \rho+1}}\right)^{\frac{1}{q}} \\
& \lesssim 2^{m \rho}\left(\sum_{k=2^{m-1}}^{2^{m}} \frac{\left|\lambda_{k}\right|^{q}}{k^{q \rho+1}}\right)^{\frac{1}{q}} \lesssim 2^{m \rho}\left(\sum_{k=1}^{2^{m}} \frac{\left|\lambda_{k}\right|^{q}}{k^{q \rho+1}}\right)^{\frac{1}{q}}=2^{m \rho} \Lambda_{2^{m}} .
\end{aligned}
$$

\subsection{Auxiliary results}

In this subsection we give some useful Lemmas that will be used in the proof our main results.

Lemma 1.5.1. [55] Let $p=1, \infty$. If $T_{2^{n}, 2^{n+1}}(x)=\sum_{\nu=2^{n}}^{2^{n+1}}\left(c_{\nu} \cos \nu x+d_{\nu} \sin \nu x\right)$, then

$$
\left\|\widetilde{T}_{2^{n}, 2^{n+1}}(x)\right\|_{p} \lesssim\left\|T_{2^{n}, 2^{n+1}}(x)\right\|_{p} \lesssim\left\|\widetilde{T}_{2^{n}, 2^{n+1}}(x)\right\|_{p} .
$$

Lemma 1.5.2. [66] Let $1 \leqslant p \leqslant \infty$ and $\alpha>0$. If $T_{n}(x)=\sum_{\nu=1}^{n}\left(c_{\nu} \cos \nu x+\right.$ $\left.d_{\nu} \sin \nu x\right)$, then

$$
n^{-\alpha}\left\|T_{n}^{(\alpha)}(x)\right\|_{p} \lesssim \omega_{\alpha}\left(T_{n}, \frac{1}{n}\right)_{p} .
$$

The following result is the well-known Marcinkiewicz multiplier theorem.

Lemma 1.5.3. [88, Ch.XV] Let $f \in L_{p}, \quad 1<p<\infty$, and (1.1.1) be the Fourier series of $f$. If $\lambda=\{\lambda\}_{n=1}^{\infty}$ satisfies the following conditions

$$
\begin{gathered}
\left|\lambda_{\nu}\right| \leqslant M, \\
\sum_{s=2^{\nu}}^{2^{\nu+1}-1}\left|\lambda_{s}-\lambda_{s+1}\right| \leqslant M \quad(\nu=0,1,2, \ldots),
\end{gathered}
$$

then there exists a function $\varphi \in L_{p}$ with the Fourier series $\sigma(f, \lambda, \beta)$ and

$$
\|\varphi\|_{p} \leqslant C(M, p)\|f\|_{p} .
$$


The following result is the well-known Hardy-Littlewood fractional integration theorem.

Lemma 1.5.4. [88, Ch.XII] Suppose that $1<p<q<\infty, \quad \theta=\frac{1}{p}-\frac{1}{q}$, and $T_{n}(x)=\sum_{\nu=1}^{n}\left(a_{\nu} \cos \nu x+b_{\nu} \sin \nu x\right)$, then

$$
\left\|T_{n}(x)\right\|_{q} \lesssim\left\|T_{n}(x)^{(\theta)}\right\|_{p}
$$

Lemma 1.5.5. (Minkowskii inequality, [43]). Let $1 \leqslant p<\infty$ and $a_{\nu k} \geqslant 0$.

Then

$$
\begin{aligned}
& \text { (a) }\left(\sum_{k=1}^{\infty}\left(\sum_{\nu=1}^{k} a_{\nu k}\right)^{p}\right)^{\frac{1}{p}} \leqslant \sum_{\nu=1}^{\infty}\left(\sum_{k=\nu}^{\infty} a_{\nu k}^{p}\right)^{\frac{1}{p}}, \\
& \text { (b) }\left(\sum_{k=1}^{\infty}\left(\sum_{\nu=k}^{\infty} a_{\nu k}\right)^{p}\right)^{\frac{1}{p}} \leqslant \sum_{\nu=1}^{\infty}\left(\sum_{k=1}^{\nu} a_{\nu k}^{p}\right)^{\frac{1}{p}} .
\end{aligned}
$$

Lemma 1.5.6. [43]. For a function $f(u, y)$, defined on measurable set $E=$ $E_{1} \times E_{2} \subset \mathbb{R}_{n}$, where $x=(u, y), u=\left(x_{1}, \ldots, x_{m}\right), y=\left(x_{m+1}, \ldots, x_{n}\right)$, the following inequality

$$
\left(\int_{E_{1}}\left|\int_{E_{2}} f(u, y) d y\right|^{p} d u\right)^{\frac{1}{p}} \leqslant \int_{E_{2}}\left(\int_{E_{1}}|f(u, y)|^{p} d u\right)^{\frac{1}{p}} d y
$$

holds for those $p \geqslant 1$ for which the right part of this inequality is finite.

Lemma 1.5.7. [88]. Let $f \in L_{p}, 1<p<\infty, n \in \mathbb{N} \cup\{0\}(i=1,2)$. Then

$$
\begin{gathered}
\left\|S_{n}(f)\right\|_{p} \leqslant C\|f\|_{p}, \\
C_{1}\left\|f-S_{n}(f)\right\|_{p} \leqslant E_{n}(f)_{p} \leqslant C_{2}\left\|f-S_{n}(f)\right\|_{p} .
\end{gathered}
$$

We denote $\Delta_{0}=A_{0}(x), \Delta_{m}=\sum_{n=2^{m-1}}^{2^{m}-1} A_{n}(x), m \in \mathbb{N}$.

Lemma 1.5.8. [88].

(a). Let $f \in L_{p}, 1<p<\infty$. Then

$$
\left(\int_{0}^{2 \pi}\left(\sum_{\nu=0}^{\infty} \Delta_{\nu}^{2}\right)^{\frac{p}{2}} d x\right)^{\frac{1}{p}} \leqslant C(p)\|f\|_{p}
$$


(b). Let $1<p<\infty$. If (1.1.1) satisfies the following inequality

$$
I_{p}=\left(\int_{0}^{2 \pi} \int_{0}^{2 \pi}\left(\sum_{\nu=0}^{\infty} \Delta_{\nu}^{2}\right)^{\frac{p}{2}} d x\right)^{\frac{1}{p}}<\infty
$$

Then (1.1.1) is the Fourier series of a function $f(x) \in L_{p}$ and $\|f\|_{p} \leqslant C(p) I_{p}$.

We will also need the following technical lemma.

Lemma 1.5.9. Let $\alpha \in \mathbb{R}_{+}, \beta \in \mathbb{R}, \rho \in \mathbb{R}_{+} \cup\{0\}$. Let $f \in L_{p}, 1 \leqslant p \leqslant \infty$ and $S_{n}(f)$ be the $n$-th partial sum of Fourier series of $f$. In particular,

$$
S_{n}\left(f^{(\lambda, \beta)}\right)=\sum_{m=1}^{n} \lambda_{m}\left(a_{m} \cos \left(m x+\frac{\pi \beta}{2}\right)+b_{m} \sin \left(m x+\frac{\pi \beta}{2}\right)\right) .
$$

Then the following inequality holds

$$
\begin{aligned}
& \left\|S_{2^{n}}^{(\alpha)}\left(f^{(\lambda, \beta)}\right)\right\|_{p} \leqslant\left|\cos \frac{\pi(\beta-\rho)}{2}\right|\left\|S_{2^{n}}^{(\alpha+\rho)}\left(f^{\left(\frac{\lambda n}{n^{\rho}}, 0\right)}\right)\right\|_{p} \\
& +\left|\sin \frac{\pi(\beta-\rho)}{2}\right|\left\|\widetilde{S}_{2^{n}}^{(\alpha+\rho)}\left(f^{\left(\frac{\lambda n}{n^{\rho}}, 0\right)}\right)\right\|_{p} .
\end{aligned}
$$

Proof. We have

$$
\begin{aligned}
& S_{2^{n}}^{(\alpha)}\left(f^{(\lambda, \beta)}\right)=\left(\sum_{m=1}^{n} \lambda_{m}\left[a_{m} \cos \left(m x+\frac{\pi \beta}{2}\right)+b_{m} \sin \left(m x+\frac{\pi \beta}{2}\right)\right]\right)^{(\alpha)} \\
& =\left(\sum_{m=1}^{n} \lambda_{m}\left[a_{m} \cos \left(m x+\frac{\pi(\beta-\rho)}{2}+\frac{\pi \rho}{2}\right)+b_{m} \sin \left(m x+\frac{\pi(\beta-\rho)}{2}+\frac{\pi \rho}{2}\right)\right]\right)^{(\alpha)} \\
& =\left(\left(\sum_{m=1}^{n} \frac{\lambda_{m}}{m^{\rho}} m^{\rho}\left[a_{m} \cos \left(m x+\frac{\pi \rho}{2}\right)+b_{m} \sin \left(m x+\frac{\pi \rho}{2}\right)\right]\right) \cos \frac{\pi(\beta-\rho)}{2}\right. \\
& \left.-\left(\sum_{m=1}^{n} \frac{\lambda_{m}}{m^{\rho}} m^{\rho}\left[a_{m} \sin \left(m x+\frac{\pi \rho}{2}\right)-b_{m} \cos \left(m x+\frac{\pi \rho}{2}\right)\right]\right) \sin \frac{\pi(\beta-\rho)}{2}\right)^{(\alpha)}=: I .
\end{aligned}
$$


Applying the definition of the fractional derivative, we have

$$
\begin{array}{r}
\|I\|_{p}=\|\left(\sum_{m=1}^{n} \frac{\lambda_{m}}{m^{\rho}}\left[a_{m} \cos m x+b_{m} \sin m x\right]\right)^{(\alpha+\rho)} \cos \frac{\pi(\beta-\rho)}{2} \\
-\left(\sum_{m=1}^{n} \frac{\lambda_{m}}{m^{\rho}}\left[a_{m} \sin m x-b_{m} \cos m x\right]\right)^{(\alpha+\rho)} \sin \frac{\pi(\beta-\rho)}{2} \|_{p} \\
\leqslant\left|\cos \frac{\pi(\beta-\rho)}{2}\right|\left\|S_{2^{n}}^{(\alpha+\rho)}\left(f^{\left(\frac{\lambda n}{n^{\rho}}, 0\right)}\right)\right\|_{p}+\left|\sin \frac{\pi(\beta-\rho)}{2}\right|\left\|\widetilde{S}_{2^{n}}^{(\alpha+\rho)}\left(f^{\left(\frac{\lambda n}{n^{p}}, 0\right)}\right)\right\|_{p} .
\end{array}
$$

The proof is now complete.

\subsection{Estimates of best approximations and moduli of smooth-} ness for generalized Liouville - Weyl derivatives

In this subsection, we obtain estimates of norms, best approximations, and moduli of smoothness in $L_{p}, 1<p<\infty$, of the generalized LiouvilleWeyl derivatives via the best approximation and moduli of smoothness of the function itself. The main results of this section were published in [30]. For the sake of convenience, we assume that $[\xi]=1$ for $0<\xi<1$.

Theorem 1.6.1. Let $1<p<\infty, \theta=\min (2, p), \quad \lambda=\left\{\lambda_{n}\right\}_{n=1}^{\infty} \in G M$, $\alpha \in \mathbb{R}_{+}$, and $r \in \mathbb{R}_{+} \cup\{0\}$. If $f \in L_{p}$ and the series

$$
\sum_{n=1}^{\infty}\left|\lambda_{n+1}^{\theta}-\lambda_{n}^{\theta}\right| E_{n}^{\theta}(f)_{p}
$$

converges, then there exists a function $f^{(\lambda, \beta)} \in L_{p}$ with the Fourier series $\sigma(f, \lambda, \beta)$ and

$$
\begin{gathered}
\left\|f^{(\lambda, \beta)}\right\|_{p} \lesssim\left\{\lambda_{1}^{\theta} E_{0}^{\theta}(f)_{p}+\sum_{n=1}^{\infty}\left|\lambda_{n+1}^{\theta}-\lambda_{n}^{\theta}\right| E_{n}^{\theta}(f)_{p}\right\}^{\frac{1}{\theta}}, \\
E_{n}\left(f^{(\lambda, \beta)}\right)_{p} \lesssim\left\{\lambda_{[n / 2]}^{\theta} E_{[n / 2]}^{\theta}(f)_{p}+\sum_{k=[n / 4]}^{\infty}\left|\lambda_{k+1}^{\theta}-\lambda_{k}^{\theta}\right| E_{k}^{\theta}(f)_{p}\right\}^{\frac{1}{\theta}},
\end{gathered}
$$


and

$$
\begin{aligned}
\omega_{\alpha}\left(f^{(\lambda, \beta)}, \frac{1}{n}\right)_{p} \lesssim\left\{n^{-\alpha \theta} \sum_{k=1}^{n}\left|\frac{\lambda_{k}^{\theta}}{k^{r \theta}}-\frac{\lambda_{k+1}^{\theta}}{(k+1)^{r \theta}}\right| k^{(r+\alpha) \theta} \omega_{\alpha+r}^{\theta}\left(f, \frac{1}{k}\right)_{p}\right. \\
\left.+\sum_{k=n+1}^{\infty}\left|\lambda_{k+1}^{\theta}-\lambda_{k}^{\theta}\right| \omega_{\alpha+r}^{\theta}\left(f, \frac{1}{k}\right)_{p}+\lambda_{n}^{\theta} \omega_{\alpha+r}^{\theta}\left(f, \frac{1}{n}\right)_{p}\right\}^{\frac{1}{\theta}} .
\end{aligned}
$$

Remark 1.6.2. In fact, we will obtain a sharper inequality than (1.6.30):

$$
E_{2^{m}-1}\left(f^{(\lambda, \beta)}\right)_{p} \lesssim\left\{\lambda_{2^{m}}^{\theta} E_{2^{m}-1}^{\theta}(f)_{p}+\sum_{\nu=m+1}^{\infty}\left|\lambda_{2^{\nu-1}}^{\theta}-\lambda_{2^{\nu-2}}^{\theta}\right| E_{2^{\nu-1}-1}^{\theta}(f)_{p}\right\}^{\frac{1}{\theta}} .
$$

Remark 1.6.3. Since any decreasing sequence or any increasing sequence satisfying the $\Delta_{2}$-condition belongs to the GM class, Theorem 1.6.1 extends Theorem A1.

Analogues of Theorems 1.6.1 for the class $G M^{\uparrow}$ can be written as follows.

Theorem 1.6.1'. Let $1<p<\infty, \theta=\min (2, p), \quad \lambda=\left\{\lambda_{n}\right\}_{n=1}^{\infty} \in G M^{\uparrow}$, $\alpha \in \mathbb{R}_{+}, r \geqslant 0$. If $f \in L_{p}$ and

$$
\sum_{n=1}^{\infty}\left|\lambda_{2^{n}}^{\theta}-\lambda_{2^{n-1}}^{\theta}\right| E_{2^{n-1}}^{\theta}(f)_{p}<\infty,
$$

then there exists a function $f^{(\lambda, \beta)} \in L_{p}$ with the Fourier series $\sigma(f, \lambda, \beta)$ and

$$
\begin{aligned}
& \left\|f^{(\lambda, \beta)}\right\|_{p} \lesssim\left\{\lambda_{1}^{\theta} E_{0}^{\theta}(f)_{p}+\sum_{n=1}^{\infty}\left|\lambda_{2^{n}}^{\theta}-\lambda_{2^{n-1}}^{\theta}\right| E_{2^{n-1}}^{\theta}(f)_{p}\right\}^{\frac{1}{\theta}}, \\
& E_{2^{n}}\left(f^{(\lambda, \beta)}\right)_{p} \lesssim\left\{\lambda_{2^{n}}^{\theta} E_{2^{n-1}}^{\theta}(f)_{p}+\sum_{k=n}^{\infty}\left|\lambda_{2^{k}}^{\theta}-\lambda_{2^{k-1}}^{\theta}\right| E_{2^{k-1}}^{\theta}(f)_{p}\right\}^{\frac{1}{\theta}},
\end{aligned}
$$

and

$$
\begin{aligned}
\omega_{\alpha}\left(f^{(\lambda, \beta)}, \frac{1}{2^{n}}\right)_{p} \lesssim & \left\{2^{-n \alpha \theta} \sum_{\nu=1}^{n} 2^{(r+\alpha) \nu \theta}\left|\frac{\lambda_{2^{\nu+1}}^{\theta}}{2^{(\nu+1) r \theta}}-\frac{\lambda_{2^{\nu}}^{\theta}}{2^{\nu r \theta}}\right| \omega_{\alpha+r}^{\theta}\left(f, \frac{1}{2^{\nu}}\right)_{p}\right. \\
& \left.+\sum_{\nu=n+2}^{\infty}\left|\lambda_{2^{\nu}}^{\theta}-\lambda_{2^{\nu-1}}^{\theta}\right| \omega_{\alpha+r}^{\theta}\left(f, \frac{1}{2^{\nu}}\right)_{p}+\lambda_{2^{n}}^{\theta} \omega_{\alpha+r}^{\theta}\left(f, \frac{1}{2^{n}}\right)_{p}\right\}^{\frac{1}{\theta}} .
\end{aligned}
$$


Proof of Theorem 1.6.1. Let $f \in L_{p}$ and series (1.6.28) be convergent. We use the following inequality

$$
\left|\lambda_{2^{n-1}}^{\theta}\right| \leqslant\left|\lambda_{1}^{\theta}\right|+\sum_{\nu=2}^{n}\left|\lambda_{2^{\nu-1}}^{\theta}-\lambda_{2^{\nu-2}}^{\theta}\right|
$$

Recall that $\Delta_{1}=A_{1}(f, x), \Delta_{n}=\sum_{2^{n-1}}^{2^{n}-1} A_{\nu}(f, x), n=2,3, \ldots$ Using inequality (1.6.32) and the inequality

$$
C_{1}(\alpha)\left(u_{1}^{\alpha}+u_{2}^{\alpha}\right) \leqslant\left(u_{1}+u_{2}\right)^{\alpha} \leqslant C_{2}(\alpha)\left(u_{1}^{\alpha}+u_{2}^{\alpha}\right)
$$

for $\alpha>0$ and $u_{1}, u_{1} \geqslant 0$, we get

$$
\begin{aligned}
I_{1} & =\left\{\int_{0}^{2 \pi}\left[\sum_{n=1}^{\infty}\left|\lambda_{2^{n-1}}^{2}\right| \Delta_{n}^{2}\right]^{\frac{p}{2}} d x\right\}^{\frac{1}{p}} \\
& \leqslant\left\{\int_{0}^{2 \pi}\left[\left|\lambda_{1}^{2}\right| \Delta_{1}^{2}+\sum_{n=2}^{\infty} \Delta_{n}^{2}\left[\lambda_{1}^{\theta}+\sum_{\nu=2}^{n}\left|\lambda_{2^{\nu-1}}^{\theta}-\lambda_{2^{\nu-2}}^{\theta}\right|\right]^{\frac{2}{\theta}}\right]^{\frac{p}{2}} d x\right\}^{\frac{1}{p}} \\
& \lesssim\left\{\int_{0}^{2 \pi}\left\{\left[\sum_{n=1}^{\infty}\left|\lambda_{1}^{2}\right| \Delta_{n}^{2}\right]^{\frac{\theta}{2}}+\left[\sum_{n=2}^{\infty}\left(\sum_{\nu=2}^{n} \Delta_{n}^{\theta}\left|\lambda_{2^{\nu-1}}^{\theta}-\lambda_{2^{\nu-2}}^{\theta}\right|\right)^{\frac{2}{\theta}}\right]^{\frac{\theta}{2}}\right\}^{\frac{p}{\theta}} d x\right\}^{\frac{1}{p}}
\end{aligned}
$$

Further, applying again Minkowski's inequality (Lemma 1.5.5) for $\frac{2}{\theta} \geqslant 1$, we have

$$
\begin{aligned}
I_{1} & \lesssim\left\{\int_{0}^{2 \pi}\left\{\lambda_{1}^{\theta}\left[\sum_{n=1}^{\infty} \Delta_{n}^{2}\right]^{\frac{\theta}{2}}+\sum_{\nu=2}^{\infty}\left[\sum_{n=\nu}^{\infty}\left[\Delta_{n}^{\theta}\left|\lambda_{2^{\nu-1}}^{\theta}-\lambda_{2^{\nu-2}}^{\theta}\right|\right]^{\frac{2}{\theta}}\right]^{\frac{\theta}{2}}\right\}^{\frac{p}{\theta}} d x\right\}^{\frac{1}{p}} \\
& \lesssim\left\{\left(\int_{0}^{2 \pi}\left[\lambda_{1}^{\theta}\left(\sum_{n=1}^{\infty} \Delta_{n}^{2}\right)^{\frac{\theta}{2}}\right]^{\frac{p}{\theta}} d x\right)^{\frac{\theta}{p}}\right. \\
& \left.+\left(\int_{0}^{2 \pi}\left[\sum_{\nu=2}^{\infty}\left|\lambda_{2^{\nu-1}}^{\theta}-\lambda_{2^{\nu-2}}^{\theta}\right|\left(\sum_{n=\nu}^{\infty} \Delta_{n}^{2}\right)^{\frac{\theta}{2}}\right]^{\frac{p}{\theta}} d x\right)^{\frac{\theta}{p}}\right\}^{\frac{1}{\theta}} .
\end{aligned}
$$

Using Minkowski's inequality with $\frac{p}{\theta} \geqslant 1$, the Littlewood-Paley theorem ([88, Ch.15]), and the following inequality $\left\|f-S_{n}(f)\right\|_{p} \lesssim E_{n}(f)_{p}$ ([8, p. 207]), we 
obtain

$$
I_{1} \lesssim\left\{\lambda_{1}^{\theta} E_{0}^{\theta}(f)_{p}+\sum_{\nu=1}^{\infty}\left|\lambda_{2^{\nu}}^{\theta}-\lambda_{2^{\nu-1}}^{\theta}\right| E_{2^{\nu}-1}^{\theta}(f)_{p}\right\}^{\frac{1}{\theta}}
$$

Then, convergence of the series (1.6.28) implies $I_{1}<\infty$. Hence, by the Littlewood-Paley theorem, there exists a function $g \in L_{p}$ with the Fourier series

$$
\sum_{n=1}^{\infty}\left|\lambda_{2^{n-1}}\right| \Delta_{n}
$$

and $\|g\|_{p} \lesssim I_{1}$. We rewrite series (1.6.34) in the form of $\sum_{n=1}^{\infty} \gamma_{n} A_{n}(f, x)$, where $\gamma_{1}:=\left|\lambda_{1}\right|, \gamma_{\nu}:=\left|\lambda_{2^{n-1}}\right|$ for $2^{n-1} \leqslant \nu \leqslant 2^{n}-1 \quad(n=2,3, \ldots)$. Consider

$$
\sum_{n=1}^{\infty} \lambda_{n} A_{n}(f, x)=\sum_{n=1}^{\infty} \gamma_{n} \Lambda_{n} A_{n}(f, x),
$$

where $\Lambda_{1}:=1, \Lambda_{\nu}:=\frac{\left|\lambda_{\nu}\right|}{\gamma_{n}}=\frac{\left|\lambda_{\nu}\right|}{\left|\lambda_{2^{n-1}}\right|}$ for $2^{n-1} \leqslant \nu \leqslant 2^{n}-1(n=2,3, \ldots)$. Since the sequence $\Lambda=\left\{\Lambda_{\nu}\right\}_{\nu=1}^{\infty}$ satisfies the conditions of the Marcinkiewicz theorem (see Lemma 1.5.3), then series (1.6.35) is the Fourier series of a function $f^{(\lambda, \beta)} \in L_{p}$ and

$$
\left\|f^{(\lambda, \beta)}\right\|_{p} \lesssim\|g\|_{p}
$$

Further, we estimate the right-hand side of (1.6.33) as follows

$$
\begin{aligned}
\left\|f^{(\lambda, \beta)}\right\|_{p} & \lesssim\left\{\lambda_{1}^{\theta} E_{0}^{\theta}(f)_{p}+\sum_{\nu=1}^{\infty}\left|\lambda_{2^{\nu}}^{\theta}-\lambda_{2^{\nu-1}}^{\theta}\right| E_{2^{\nu}-1}^{\theta}(f)_{p}\right\}^{\frac{1}{\theta}} \\
& \lesssim\left\{\lambda_{1}^{\theta} E_{0}^{\theta}(f)_{p}+\sum_{\nu=1}^{\infty} E_{2^{\nu}-1}^{\theta}(f)_{p} \sum_{n=2^{\nu-1}}^{2^{\nu}-1}\left|\lambda_{n+1}^{\theta}-\lambda_{n}^{\theta}\right|\right\}^{\frac{1}{\theta}} \\
& \lesssim\left\{\lambda_{1}^{\theta} E_{0}^{\theta}(f)_{p}+\sum_{n=1}^{\infty}\left|\lambda_{n+1}^{\theta}-\lambda_{n}^{\theta}\right| E_{n}^{\theta}(f)_{p}\right\}^{\frac{1}{\theta}} .
\end{aligned}
$$

Thus, (1.6.29) has been proved.

In the same way let us verify (1.6.30). Now we use the inequality $E_{n}\left(f^{(\lambda, \beta)}\right)_{p} \leqslant\left\|f^{(\lambda, \beta)}-S_{n}\left(f^{(\lambda, \beta)}\right)\right\|_{p}$. Applying inequality (1.6.32) and 
Minkowski's inequality, we get

$$
\begin{aligned}
& \left\{\int_{0}^{2 \pi}\left[\sum_{k=m+1}^{\infty}\left|\lambda_{2^{k-1}}^{2}\right| \Delta_{k}^{2}\right]^{\frac{p}{2}} d x\right\}^{\frac{1}{p}} \\
& \leqslant\left\{\int_{0}^{2 \pi}\left[\sum_{k=m+1}^{\infty}\left(\lambda_{2^{m}}^{\theta}+\sum_{\nu=m+1}^{k}\left|\lambda_{2^{\nu-1}}^{\theta}-\lambda_{2^{\nu-2}}^{\theta}\right|\right)^{\frac{2}{\theta}} \Delta_{k}^{2}\right]^{\frac{p}{2}} d x\right\}^{\frac{1}{p}} \\
& \leqslant\left\{\lambda_{2^{m}}^{\theta}\left(\int_{0}^{2 \pi}\left[\sum_{k=m+1}^{\infty} \Delta_{k}^{2}\right]^{\frac{p}{2}} d x\right)^{\frac{\theta}{p}}\right. \\
& \left.+\sum_{\nu=m+1}^{\infty}\left|\lambda_{2^{\nu-1}}^{\theta}-\lambda_{2^{\nu-2}}^{\theta}\right|\left(\int_{0}^{2 \pi}\left[\sum_{k=\nu}^{\infty} \Delta_{k}^{2}\right]^{\frac{p}{2}} d x\right)^{\frac{\theta}{p}}\right\}^{\frac{1}{\theta}}=I_{2} .
\end{aligned}
$$

By the Littlewood-Paley theorem, we have

$$
\begin{aligned}
I_{2} & \lesssim\left\{\lambda_{2^{m}}^{\theta} E_{2^{m}-1}^{\theta}(f)_{p}+\sum_{\nu=m+1}^{\infty}\left|\lambda_{2^{\nu-1}}^{\theta}-\lambda_{2^{\nu-2}}^{\theta}\right| E_{2^{\nu-1}-1}^{\theta}(f)_{p}\right\}^{\frac{1}{\theta}} \\
& \lesssim\left\{\lambda_{\left[\frac{n}{2}\right]}^{\theta} E_{\left[\frac{n}{2}\right]}^{\theta}(f)_{p}+\sum_{k=\left[\frac{n}{4}\right]}^{\infty}\left|\lambda_{k+1}^{\theta}-\lambda_{k}^{\theta}\right| E_{k}^{\theta}(f)_{p}\right\}^{\frac{1}{\theta}},
\end{aligned}
$$

for the given $m \in \mathbb{N}$. Now we choose $n$ such that $2^{m} \leqslant n \leqslant 2^{m+1}$. Then two previous inequalities imply (1.6.30).

Finally, let us prove (1.6.31). Taking into account Lemma 1.2.3, we get

$$
\omega_{\alpha}\left(f^{(\lambda, \beta)}, \frac{1}{n}\right)_{p} \lesssim\left\|f^{(\lambda, \beta)}-S_{n}\left(f^{(\lambda, \beta)}\right)\right\|_{p}+n^{-\alpha}\left\|S_{n}^{(\alpha)}\left(f^{(\lambda, \beta)}\right)\right\|_{p} .
$$

Let us estimate the first term. Applying Jackson's inequality to estimate (1.6.36), we obtain

$\left\|f^{(\lambda, \beta)}-S_{n}\left(f^{(\lambda, \beta)}\right)\right\|_{p} \lesssim\left\{\lambda_{[n / 2]}^{\theta} \omega_{\alpha+r}^{\theta}\left(f, \frac{2}{n}\right)_{p}+\sum_{s=[n / 4]}^{\infty}\left|\lambda_{s+1}^{\theta}-\lambda_{s}^{\theta}\right| \omega_{\alpha+r}^{\theta}\left(f, \frac{1}{s}\right)_{p}\right\}^{\frac{1}{\theta}}$

To estimate the second term in (1.6.37), we use the same reasoning as in (1.6.29) and the following inequality

$$
2^{-s r \theta}\left|\lambda_{2^{s}}^{\theta}\right| \leqslant 2^{-m r \theta}\left|\lambda_{2^{m}}^{\theta}\right|+\sum_{\nu=s}^{m-1}\left|2^{-\nu r \theta} \lambda_{2^{\nu}}^{\theta}-2^{-(\nu+1) r \theta} \lambda_{2^{\nu+1}}^{\theta}\right|
$$


Using this inequality, we obtain

$$
\begin{aligned}
I_{3}= & \left\{\int_{0}^{2 \pi}\left[\sum_{s=1}^{m}\left|\lambda_{2^{s-1}}^{2}\right| 2^{2 \alpha(s-1)} \Delta_{s}^{2}\right]^{\frac{p}{2}} d x\right\}^{\frac{1}{p}} \\
\leqslant & \left\{\int _ { 0 } ^ { 2 \pi } \left[\sum _ { s = 1 } ^ { m } \left(2^{-m r \theta} \lambda_{2^{m}}^{\theta}+\right.\right.\right. \\
& \left.\left.\left.\quad+\sum_{\nu=s-1}^{m-1}\left|2^{-\nu r \theta} \lambda_{2^{\nu}}^{\theta}-2^{-(\nu+1) r \theta} \lambda_{2^{\nu+1}}^{\theta}\right|\right)^{\frac{2}{\theta}} 2^{2(s-1)(\alpha+r)} \Delta_{s}^{2}\right]^{\frac{p}{2}} d x\right\}^{\frac{1}{p}} .
\end{aligned}
$$

By Minkowski's inequality,

$$
\begin{aligned}
I_{3} & \leqslant\left\{\left(\int_{0}^{2 \pi}\left[2^{-m r \theta} \lambda_{2^{m}}^{\theta}\left[\sum_{s=1}^{m} 2^{2(s-1)(\alpha+r)} \Delta_{s}^{2}\right]^{\frac{\theta}{2}}\right]^{\frac{p}{\theta}} d x\right)^{\frac{\theta}{p}}\right. \\
& \left.+\sum_{\nu=0}^{m-1}\left|2^{-\nu r \theta} \lambda_{2^{\nu}}^{\theta}-2^{-(\nu+1) r \theta} \lambda_{2^{\nu+1}}^{\theta}\right|\left(\int_{0}^{2 \pi}\left[\sum_{s=1}^{\nu+1} 2^{2(s-1)(\alpha+r)} \Delta_{s}^{2}\right]^{\frac{p}{2}} d x\right)^{\frac{\theta}{p}}\right\}^{\frac{1}{\theta}}=I_{4} .
\end{aligned}
$$

By the Littlewood-Paley theorem, we have

$$
\left(\int_{0}^{2 \pi}\left[\sum_{s=1}^{m} 2^{2(s-1)(\alpha+r)} \Delta_{s}^{2}\right]^{\frac{p}{2}} d x\right)^{\frac{1}{p}} \lesssim\left\|S_{2^{m}-1}^{(\alpha+r)}(f)\right\|_{p} .
$$

From Lemma 1.5.2, we obtain

$$
\begin{aligned}
\left\|S_{2^{m}-1}^{(\alpha+r)}(f)\right\|_{p} & \lesssim 2^{m(\alpha+r)} \omega_{\alpha+r}\left(S_{2^{m}-1} \pm f, \frac{1}{2^{m}-1}\right)_{p} \\
& \lesssim 2^{m(\alpha+r)}\left(\omega_{\alpha+r}\left(f, \frac{1}{2^{m}-1}\right)_{p}+\left\|S_{2^{m}-1}-f\right\|_{p}\right) \\
& \lesssim 2^{m(\alpha+r)}\left(\omega_{\alpha+r}\left(f, \frac{1}{2^{m}-1}\right)_{p}+E_{2^{m}}(f)_{p}\right) \\
& \lesssim 2^{m(\alpha+r)} \omega_{\alpha+r}\left(f, \frac{1}{2^{m}-1}\right)_{p}
\end{aligned}
$$

Therefore,

$$
\begin{aligned}
I_{4} & \lesssim\left\{2^{-m r \theta} \lambda_{2^{m}}^{\theta} 2^{m(\alpha+r) \theta} \omega_{\alpha+r}^{\theta}\left(f, \frac{1}{2^{m}-1}\right)_{p}\right. \\
& \left.+\sum_{\nu=0}^{m-1}\left|2^{-\nu r \theta} \lambda_{2^{\nu}}^{\theta}-2^{-(\nu+1) r \theta} \lambda_{2^{\nu+1}}^{\theta}\right| 2^{(\nu+1)(\alpha+r) \theta} \omega_{\alpha+r}^{\theta}\left(f, \frac{1}{2^{\nu+1}-1}\right)_{p}\right\}^{\frac{1}{\theta}} .
\end{aligned}
$$


Using monotonicity properties of moduli of smoothness and the formula

$$
\frac{\lambda_{2^{\nu}}^{\theta}}{2^{\nu \theta r}}-\frac{\lambda_{2^{\nu+1}}^{\theta}}{2^{(\nu+1) \theta r}}=\sum_{k=2^{\nu}}^{2^{\nu+1}-1}\left(\frac{\lambda_{k}^{\theta}}{k^{\theta r}}-\frac{\lambda_{k+1}^{\theta}}{(k+1)^{\theta r}}\right),
$$

we have

$$
\begin{aligned}
I_{4} & \lesssim\left\{2^{\alpha m \theta} \lambda_{2^{m}}^{\theta} \omega_{\alpha+r}^{\theta}\left(f, \frac{1}{2^{m}-1}\right)_{p}\right. \\
& \left.+2^{(\alpha+r) \theta} \sum_{k=1}^{2^{m}-1}\left|k^{-r \theta} \lambda_{k}^{\theta}-(k+1)^{-r \theta} \lambda_{k+1}^{\theta}\right| k^{(\alpha+r)} \omega_{\alpha+r}^{\theta}\left(f, \frac{1}{k}\right)_{p}\right\}^{\frac{1}{\theta}} .
\end{aligned}
$$

Combining estimates for $I_{3}$ and $I_{4}$, we get

$$
\begin{aligned}
& n^{-\alpha}\left\|S_{n}^{(\alpha)}\left(f^{(\lambda, \beta)}\right)\right\|_{p} \lesssim\left\{\lambda_{[n / 2]}^{\theta} \omega_{\alpha+r}^{\theta}\left(f, \frac{2}{n}\right)_{p}\right. \\
& \left.+n^{-\alpha \theta} \sum_{\nu=1}^{n}\left|\nu^{-r \theta} \lambda_{\nu}^{\theta}-(\nu+1)^{-r \theta} \lambda_{\nu+1}^{\theta}\right| \nu^{(r+\alpha) \theta} \omega_{\alpha+r}^{\theta}\left(f, \frac{1}{\nu}\right)_{p}\right\}^{\frac{1}{\theta}} .
\end{aligned}
$$

From (1.6.38) and (1.6.39), we have

$$
\begin{aligned}
& \omega_{\alpha}\left(f^{(\lambda, \beta)}, \frac{1}{n+1}\right)_{p} \\
& \lesssim\left\{n^{-\alpha \theta} \sum_{k=1}^{n} k^{(r+\alpha) \theta}\left|k^{-r \theta} \lambda_{k}^{\theta}-(k+1)^{-r \theta} \lambda_{k+1}^{\theta}\right| \omega_{\alpha+r}^{\theta}\left(f, \frac{1}{k}\right)_{p}\right. \\
& \left.+\sum_{k=[n / 4]}^{\infty}\left|\lambda_{k+1}^{\theta}-\lambda_{k}^{\theta}\right| \omega_{\alpha+r}^{\theta}\left(f, \frac{1}{k}\right)_{p}+\lambda_{[n / 2]}^{\theta} \omega_{\alpha+r}^{\theta}\left(f, \frac{2}{n}\right)_{p}\right\}^{\frac{1}{\theta}} .
\end{aligned}
$$

Now we note that

$$
\begin{aligned}
& \sum_{k=[n / 4]}^{n}\left|\lambda_{k+1}^{\theta}-\lambda_{k}^{\theta}\right| \omega_{\alpha+r}^{\theta}\left(f, \frac{1}{k}\right)_{p} \\
& \leqslant \sum_{k=[n / 4]}^{n}\left(\frac{\lambda_{k+1}^{\theta}}{(k+1)^{r \theta}} k^{r \theta-1}+k^{r \theta}\left|\frac{\lambda_{k}^{\theta}}{k^{r \theta}}-\frac{\lambda_{k+1}^{\theta}}{(k+1)^{r \theta}}\right|\right) \omega_{\alpha+r}^{\theta}\left(f, \frac{1}{k}\right)_{p} \\
& \leqslant \lambda_{[n / 4]}^{\theta} \omega_{\alpha+r}^{\theta}\left(f, \frac{4}{n}\right)_{p}+\sum_{k=[n / 4]}^{n}\left|\frac{\lambda_{k}^{\theta}}{k^{r \theta}}-\frac{\lambda_{k+1}^{\theta}}{(k+1)^{r \theta}}\right| k^{r \theta} \omega_{\alpha+r}^{\theta}\left(f, \frac{1}{k}\right)_{p} .
\end{aligned}
$$


Thus, we have

$$
\begin{aligned}
\omega_{\alpha}\left(f^{(\lambda, \beta)}, \frac{1}{n}\right)_{p} \lesssim & \left\{n^{-\alpha \theta} \sum_{k=1}^{n} k^{(r+\alpha) \theta}\left|k^{-r \theta} \lambda_{k}^{\theta}-(k+1)^{-r \theta} \lambda_{k+1}^{\theta}\right| \omega_{\alpha+r}^{\theta}\left(f, \frac{1}{k}\right)_{p}\right. \\
& \left.+\sum_{k=n+1}^{\infty}\left|\lambda_{k+1}^{\theta}-\lambda_{k}^{\theta}\right| \omega_{\alpha+r}^{\theta}\left(f, \frac{1}{k}\right)_{p}+\lambda_{[n / 4]}^{\theta} \omega_{\alpha+r}^{\theta}\left(f, \frac{1}{n}\right)_{p}\right\}^{\frac{1}{\theta}} .
\end{aligned}
$$

Nothing that

$$
\lambda_{\frac{n}{4}}^{\theta} \leqslant\left(\frac{n}{4}\right)^{r \theta} \sum_{k=[n / 4]}^{n-1}\left|\frac{\lambda_{k}^{\theta}}{k^{r \theta}}-\frac{\lambda_{k+1}^{\theta}}{(k+1)^{r \theta}}\right|+\frac{\lambda_{n}^{\theta}}{n^{r \theta}}\left(\frac{n}{4}\right)^{r \theta},
$$

we arrive at

$$
\begin{array}{r}
\omega_{\alpha}\left(f^{(\lambda, \beta)}, \frac{1}{n}\right)_{p} \lesssim\left\{n^{-\alpha \theta} \sum_{k=1}^{n} k^{(r+\alpha) \theta}\left|k^{-r \theta} \lambda_{k}^{\theta}-(k+1)^{-r \theta} \lambda_{k+1}^{\theta}\right| \omega_{\alpha+r}^{\theta}\left(f, \frac{1}{k}\right)_{p}\right. \\
\left.\quad+\sum_{k=n}^{\infty}\left|\lambda_{k+1}^{\theta}-\lambda_{k}^{\theta}\right| \omega_{\alpha+r}^{\theta}\left(f, \frac{1}{k}\right)_{p}+\lambda_{n}^{\theta} \omega_{\alpha+r}^{\theta}\left(f, \frac{1}{n}\right)_{p}\right\}^{\frac{1}{\theta}} .
\end{array}
$$

The proof of Theorem 1.6.1 is complete.

1.7 Estimates for transformed Fourier series in the limiting cases $p=1$ and $p=\infty$

In this subsection we obtain estimates of norms, best approximations, and moduli of smoothness of the generalized Liouville-Weyl derivatives in the limiting cases $p=1$ and $p=\infty$.

Theorem 1.7.1. Let $p=1$ or $p=\infty$ and $\left\{\Delta \lambda_{n}\right\} \in G M$. If $f \in L_{p}$ and the series

$$
\sum_{n=1}^{\infty}\left(\sum_{k=2^{n-1}}^{2^{n}}\left|\Delta \lambda_{k}\right|+\left|\lambda_{2^{n+2}}\right|\right) E_{2^{n}}(f)_{p}
$$

converges, then there exists a function $f^{(\lambda, \beta)} \in L_{p}$ with the Fourier series $\sigma(f, \lambda, \beta)$ such that

$$
\left\|f^{(\lambda, \beta)}\right\|_{p} \lesssim\left|\lambda_{1}\right| E_{0}(f)_{p}+\sum_{n=1}^{\infty}\left(\sum_{k=2^{n-1}}^{2^{n}}\left|\Delta \lambda_{k}\right|+\left|\lambda_{2^{n+2}}\right|\right) E_{2^{n-1}}(f)_{p}
$$


and

$$
E_{2^{n+1}}\left(f^{(\lambda, \beta)}\right)_{p} \lesssim \sum_{s=n}^{\infty}\left(\sum_{k=2^{s-1}}^{2^{s}}\left|\Delta \lambda_{k}\right|+\left|\lambda_{2^{s+2}}\right|\right) E_{2^{s-1}}(f)_{p} .
$$

Moreover, if $\left\{\Delta \frac{\lambda_{n}}{n^{\rho}}\right\} \in G M, \alpha \in \mathbb{R}_{+}$and $\rho \in \mathbb{R}_{+} \cup\{0\}$, then

$$
\begin{aligned}
\omega_{\alpha}\left(f^{(\lambda, \beta)}, \frac{1}{2^{n}}\right)_{p} & \lesssim \sum_{s=n}^{\infty}\left(\sum_{k=2^{s-1}}^{2^{s}}\left|\Delta \lambda_{k}\right|+\left|\lambda_{2^{s+2}}\right|\right) \omega_{\alpha+r}\left(f, \frac{1}{2^{s}}\right)_{p} \\
& +2^{-n \alpha} \sum_{\mu=1}^{2^{n+2}-1}\left|\frac{\lambda_{\mu}}{\mu^{\rho}}-\frac{\lambda_{\mu+1}}{(\mu+1)^{\rho}}\right| \mu^{\alpha+\rho} \omega_{\alpha+\rho}\left(f, \frac{1}{\mu}\right)_{p} \\
& +2^{-n \alpha}\left|\sin \frac{\pi(\beta-\rho)}{2}\right| \sum_{k=1}^{n}\left|\lambda_{2^{n+1}}\right| 2^{n \alpha} \omega_{\alpha+\rho}\left(f, \frac{1}{2^{n}}\right)_{p} .
\end{aligned}
$$

Remark 1.7.4. Note that the conditions $\left\{\Delta \lambda_{n}\right\} \in G M,\left\{\Delta \frac{\lambda_{n}}{n^{\rho}}\right\} \in G M$ in Theorem 1.7.1 are much weaker than the corresponding conditions on $\left\{\lambda_{n}\right\}$ in Theorem A2.

The following analogues of Theorems 1.7.1 for $G M^{\uparrow}$ can be written as follows.

Theorem 1.7.1' Let $p=1$ or $p=\infty,\left\{\Delta \lambda_{n}\right\} \in G M^{\uparrow},\left\{\Delta \frac{\lambda_{n}}{n^{\rho}}\right\} \in G M^{\uparrow}$, $\alpha \in \mathbb{R}_{+}$, and $\rho \in \mathbb{R}_{+} \cup\{0\}$. If $f \in L_{p}$ and the series

$$
\sum_{n=1}^{\infty}\left(\sum_{k=2^{n-1}}^{2^{n}}\left|\Delta \lambda_{k}\right|+\left|\lambda_{2^{n+2}}\right|\right) E_{2^{n}}(f)_{p}
$$

converges, then there exists a function $f^{(\lambda, \beta)} \in L_{p}$ with the Fourier series $\sigma(f, \lambda, \beta)$ and

$$
\begin{aligned}
& \left\|f^{(\lambda, \beta)}\right\|_{p} \lesssim\left|\lambda_{1}\right| E_{0}(f)_{p}+\sum_{n=1}^{\infty}\left(\sum_{k=2^{n-1}}^{2^{n}}\left|\Delta \lambda_{k}\right|+\left|\lambda_{2^{n+2}}\right|\right) E_{2^{n}}(f)_{p}, \\
& E_{2^{n+1}}\left(f^{(\lambda, \beta)}\right)_{p} \lesssim \sum_{s=n}^{\infty}\left(\sum_{k=2^{s-1}}^{2^{s}}\left|\Delta \lambda_{k}\right|+\left|\lambda_{2^{s+2}}\right|\right) E_{2^{s}}(f)_{p}, \\
& \omega_{\alpha}\left(f^{(\lambda, \beta)}, \frac{1}{2^{n}}\right)_{p} \lesssim \sum_{s=n}^{\infty}\left(\sum_{k=2^{s-1}}^{2^{s}}\left|\Delta \lambda_{k}\right|+\left|\lambda_{2^{s+2}}\right|\right) \omega_{\alpha+r}\left(f, \frac{1}{2^{s}}\right)_{p}
\end{aligned}
$$




$$
\begin{aligned}
& +2^{-n \alpha} \sum_{\mu=1}^{2^{n+2}-1}\left|\frac{\lambda_{\mu}}{\mu^{\rho}}-\frac{\lambda_{\mu+1}}{(\mu+1)^{\rho}}\right| \mu^{\alpha+\rho} \omega_{\alpha+\rho}\left(f, \frac{1}{\mu}\right)_{p} \\
& +2^{-n \alpha}\left|\sin \frac{\pi(\beta-\rho)}{2}\right| \sum_{k=1}^{n}\left|\lambda_{2^{n+1}}\right| 2^{n \alpha} \omega_{\alpha+\rho}\left(f, \frac{1}{2^{n}}\right)_{p} .
\end{aligned}
$$

Proof of Theorem 1.7.1. We consider the series

$$
\begin{gathered}
\cos \frac{\pi \beta}{2} V_{1}(\lambda, f)-\sin \frac{\pi \beta}{2} \widetilde{V}_{1}(\lambda, f)+ \\
\sum_{n=1}^{\infty}\left\{\cos \frac{\pi \beta}{2}\left(V_{2^{n}}(\lambda, f)-V_{2^{n-1}}(\lambda, f)\right)-\sin \frac{\pi \beta}{2}\left(\widetilde{V}_{2^{n}}(\lambda, f)-\widetilde{V}_{2^{n-1}}(\lambda, f)\right)\right\},
\end{gathered}
$$

where $V_{1}(\lambda, f):=\lambda_{1} A_{1}(f, x)$ and

$$
V_{n}=\sigma\left(\lambda, V_{n}(f)\right)=\sum_{m=1}^{n} \lambda_{m} A_{m}(f, x)+\sum_{m=n+1}^{2 n-1} \lambda_{m}\left(1-\frac{m-n}{n}\right) A_{m}(f, x)(n \geqslant 2) .
$$

Let $M>N>0$. Applying the Abel transformation twice, we get

$$
\begin{aligned}
J:=\| \sum_{n=N}^{M}\left[\left|\cos \frac{\pi \beta}{2}\right|\left(V_{2^{n+1}}(\lambda, f)-V_{2^{n}}(\lambda, f)\right)\right. \\
\left.\quad-\left|\sin \frac{\pi \beta}{2}\right|\left(\widetilde{V}_{2^{n+1}}(\lambda, f)-\widetilde{V}_{2^{n}}(\lambda, f)\right)\right] \|_{p} \\
=\| \sum_{n=N}^{M}\left\{\left|\cos \frac{\pi \beta}{2}\right|\left[\frac{1}{\pi} \int_{-\pi}^{\pi}\left(V_{2^{n+1}}(f)-V_{2^{n}}(f)\right)(x+u) \sum_{\nu=2^{n}}^{2^{n+2}}\left(\lambda_{\nu} \cos \nu u\right) d u\right]\right. \\
\left.-\left|\sin \frac{\pi \beta}{2}\right|\left[\frac{1}{\pi} \int_{-\pi}^{\pi}\left(\widetilde{V}_{2^{n+1}}(f)-\widetilde{V}_{2^{n}}(f)\right)(x+u) \sum_{\nu=2^{n}}^{2^{n+2}}\left(\lambda_{\nu} \cos \nu u\right) d u\right]\right\} \|_{p} .
\end{aligned}
$$

By Lemmas 1.4.5 and 1.5.1 and $\left\|f-V_{n}(f)\right\| \leqslant C E_{n}(f)$, we obtain

$$
\begin{aligned}
J & \lesssim \sum_{n=N}^{M}\left(\sum_{k=2^{n-1}}^{2^{n}}\left|\Delta \lambda_{k}\right|+\left|\lambda_{2^{n+2}}\right|\right)\left\|V_{2^{n+1}}(f)-V_{2^{n}}(f)\right\|_{p} \\
& \lesssim \sum_{n=N}^{M}\left(\sum_{k=2^{n-1}}^{2^{n}}\left|\Delta \lambda_{k}\right|+\left|\lambda_{2^{n+2}}\right|\right) E_{2^{n}}(f)_{p} .
\end{aligned}
$$


Then the convergence of series in (1.7.41) implies that there exists $f^{(\lambda, \beta)} \in L_{p}$ such that the series (1.7.45) converges to $f^{(\lambda, \beta)}$ in $L_{p}$. Moreover, the Fourier series of $f^{(\lambda, \beta)}$ is $\sigma(f, \alpha, \beta)$. This can be shown as in the proof of Theorem 1 (ii) in [55, pp.1379-1380].

Let us prove (1.7.43). Applying again Lemmas 1.4.5 and using the properties of $\left\{\lambda_{n}\right\}$ and the inequality $\left\|f-V_{m}(f)\right\|_{p} \leqslant C E_{m}(f)_{p}$, we obtain

$$
\begin{aligned}
J & =\left\|\sum_{s=N}^{M}\left[V_{2^{s+1}}\left(f^{(\lambda, \beta)}\right)-V_{2^{s}}\left(f^{(\lambda, \beta)}\right)\right]\right\|_{p} \\
& \lesssim \sum_{s=N}^{M}\left\|V_{2^{s+1}}(f)-V_{2^{s}}(f)\right\|_{p} \sum_{k=2^{s-1}}^{2^{s}}\left|\Delta \lambda_{k}\right|+\left\|\sum_{s=N}^{M} \lambda_{2^{s+2}}\left(V_{2^{s+1}}(f)-V_{2^{s}}(f)\right)\right\|_{p} \\
& \lesssim \sum_{s=N}^{M}\left(\sum_{k=2^{s-1}}^{2^{s}}\left|\Delta \lambda_{k}\right|+\left|\lambda_{2^{s+2}}\right|\right) E_{2^{s}}(f)_{p} .
\end{aligned}
$$

Hence,

$$
\left\|f^{(\lambda, \beta)}-V_{2^{n}}\left(f^{(\lambda, \beta)}\right)\right\|_{p} \lesssim \sum_{s=n}^{\infty}\left(\sum_{k=2^{s-1}}^{2^{s}}\left|\Delta \lambda_{k}\right|+\left|\lambda_{2^{s+2}}\right|\right) E_{2^{s}}(f)_{p} .
$$

Therefore, we have

$$
E_{2^{n}}\left(f^{(\lambda, \beta)}\right)_{p} \lesssim \sum_{s=n}^{\infty}\left(\sum_{k=2^{s-1}}^{2^{s}}\left|\Delta \lambda_{k}\right|+\left|\lambda_{2^{s+2}}\right|\right) E_{2^{s}}(f)_{p}
$$

In order to prove (1.7.44), we use inequality (1.2.9) from Lemma 1.2.3. The estimate of $\left\|f^{(\lambda, \beta)}-V_{2^{n}}\left(f^{(\lambda, \beta)}\right)\right\|_{p}$ follows from (1.7.46). Now let us estimate second term of (1.2.9). By Lemma 1.5.9, we have

$$
\begin{aligned}
\left\|V_{2^{n}}^{(\alpha)}\left(f^{(\lambda, \beta)}\right)\right\|_{p} & \leqslant\left|\cos \frac{\pi(\beta-\rho)}{2}\right|\left\|V_{2^{n}}^{(\alpha+\rho)}\left(f^{\left(\frac{\lambda n}{n^{\rho}}, 0\right)}\right)\right\|_{p} \\
& +\left|\sin \frac{\pi(\beta-\rho)}{2}\right|\left\|\widetilde{V}_{2^{n}}^{(\alpha+\rho)}\left(f^{\left(\frac{\lambda n}{n^{\rho}}, 0\right)}\right)\right\|_{p}
\end{aligned}
$$


Applying Abel transformation twice, we get

$$
\begin{aligned}
& \left\|V_{2^{n}}^{(\alpha+\rho)}\left(f^{\left(\frac{\lambda n}{n^{\rho}}, 0\right)}\right)\right\|_{p} \\
& =\left\|\sum_{k=0}^{n-1}\left(V_{2^{k+1}}^{(\alpha+\rho)}\left(f^{\left(\frac{\lambda_{k}}{k^{p}}, 0\right)}\right)-V_{2^{k}}^{(\alpha+\rho)}\left(f^{\left(\frac{\lambda_{k}}{k^{p}}, 0\right)}\right)\right)+V_{1}^{(\alpha+\rho)}\left(f^{\left(\lambda_{1}, 0\right)}\right)\right\|_{p} \\
& =\left\|\sum_{k=0}^{n-1} \frac{1}{\pi} \int_{-\pi}^{\pi}\left(V_{2^{k+1}}^{(\alpha+\rho)}-V_{2^{k}}^{(\alpha+\rho)}\right)(x+u) \sum_{\nu=2^{k}} \frac{\lambda_{\nu}}{\nu^{\rho}} \cos \nu u d u+\lambda_{1} V_{1}^{(\alpha+\rho)}(f)\right\|_{p} \\
& \lesssim \sum_{k=0}^{n-1}\left\|V_{2^{k+1}}^{(\alpha+\rho)}-V_{2^{k}}^{(\alpha+\rho)}\right\|_{p}\left(\sum_{j=2^{k}}^{2^{k+2}-1}\left|\Delta^{2}\left(\frac{\lambda_{j}}{j^{\rho}}\right)\right|(j+1)+2^{k+2}\left|\Delta \frac{\lambda_{2^{k+2}}}{2^{(k+2) \rho}}\right|+2^{k}\left|\Delta \frac{\lambda_{2^{k}}}{2^{k \rho}}\right|\right) \\
& +\left\|\sum_{k=0}^{n-1} \frac{\lambda_{2^{k+2}}}{2^{(k+2) \rho}}\left(V_{2^{k+1}}^{(\alpha+\rho)}-V_{2^{k}}^{(\alpha+\rho)}\right)(f)+\lambda_{1} V_{1}^{(\alpha+\rho)}(f)\right\|_{p}=: J_{1}+J_{2} .
\end{aligned}
$$

To obtain the upper estimate of $J_{1}$, we will use the properties of GM sequences (see Lemma 1.4.1)

$$
\begin{aligned}
J_{1} & \lesssim \sum_{k=0}^{n-1}\left\|V_{2^{k+1}}^{(\alpha+\rho)}-V_{2^{k}}^{(\alpha+\rho)}\right\|_{p}\left(2^{k+2} \sum_{j=2^{k}}^{2^{k+2}-1}\left|\Delta^{2}\left(\frac{\lambda_{j}}{j^{\rho}}\right)\right|+2^{k+2}\left|\Delta \frac{\lambda_{2^{k+2}}}{2^{(k+2) \rho}}\right|+2^{k}\left|\Delta \frac{\lambda_{2^{k}}}{2^{k \rho}}\right|\right) \\
& \lesssim \sum_{k=0}^{n-1}\left\|V_{2^{k+1}}^{(\alpha+\rho)}-V_{2^{k}}^{(\alpha+\rho)}\right\|_{p} 2^{k}\left|\Delta \frac{\lambda_{2^{k}}}{2^{k \rho}}\right| \\
& \lesssim \sum_{k=0}^{n-1}\left\|V_{2^{k+1}}^{(\alpha+\rho)}-V_{2^{k}}^{(\alpha+\rho)}\right\|_{p} \sum_{\nu=\left[2^{k-1}\right]}^{2^{k}}\left|\Delta \frac{\lambda_{\nu}}{\nu^{k \rho}}\right| \\
& \lesssim \sum_{\nu=1}^{2^{n-1}}\left|\frac{\lambda_{\nu}}{\nu^{\rho}}-\frac{\lambda_{\nu+1}}{(\nu+1)^{\rho}}\right| \nu^{\alpha+\rho} \omega_{\alpha+\rho}\left(f, \frac{1}{\nu}\right)_{p},
\end{aligned}
$$

where in the last step we have used Lemma 1.2.3 and monotonicity properties of modulus of smoothness. Now we estimate $J_{2}$ :

$$
\begin{gathered}
J_{2} \leqslant \| \sum_{k=0}^{n-1}\left(V_{2^{k+1}}^{(\alpha+\rho)}-V_{2^{k}}^{(\alpha+\rho)}\right)(f)\left(\sum_{\nu=k}^{n-1}\left(\frac{\lambda_{2^{\nu+2}}}{2^{(\nu+2) \rho}}-\frac{\lambda_{2^{\nu+3}}}{2^{(\nu+3) \rho}}\right)+\frac{\lambda_{2^{n+2}}}{2^{(n+2) \rho}}\right) \\
+\lambda_{1} V_{1}^{(\alpha+\rho)}(f) \|_{p} .
\end{gathered}
$$

Changing the order of summation, we derive 


$$
\begin{aligned}
J_{2} \leqslant & \| \frac{\lambda_{2^{n+2}}}{2^{(n+2) \rho}}\left(V_{2^{n}}^{(\alpha+\rho)}-V_{1}^{(\alpha+\rho)}\right)(f) \\
& +\sum_{\nu=0}^{n-1}\left(\frac{\lambda_{2^{\nu+2}}}{2^{(\nu+2) \rho}}-\frac{\lambda_{2^{\nu+3}}}{2^{(\nu+3) \rho}}\right)\left(V_{2^{\nu+1}}^{(\alpha+\rho)}-V_{1}^{(\alpha+\rho)}\right)(f)+\lambda_{1} V_{1}^{(\alpha+\rho)}(f) \|_{p} \\
& \lesssim \sum_{\nu=0}^{n-1}\left|\frac{\lambda_{2^{\nu+2}}}{2^{(\nu+2) \rho}}-\frac{\lambda_{2^{\nu+3}}}{2^{(\nu+3) \rho}}\right|\left\|V_{2^{\nu+1}}^{(\alpha+\rho)}(f)\right\|_{p} \\
+ & \left|\frac{\lambda_{2^{n+2}}}{2^{(n+2) \rho}}\right|\left\|V_{2^{n}}^{(\alpha+\rho)}\right\|_{p}+\left|\lambda_{1}\right|\left\|V_{1}^{(\alpha+\rho)}(f)\right\|_{p} \\
& \lesssim \sum_{\mu=1}^{2^{n+2}-1}\left|\frac{\lambda_{\mu}}{\mu^{\rho}}-\frac{\lambda_{\mu+1}}{(\mu+1)^{\rho}}\right| \mu^{\alpha+\rho} \omega_{\alpha+\rho}\left(f, \frac{1}{\mu}\right)_{p}+\left|\lambda_{2^{n+2}}\right| 2^{n \alpha} \omega_{\alpha+\rho}\left(f, \frac{1}{2^{n}}\right)_{p}
\end{aligned}
$$

Here we used that $\left|\lambda_{1}\right| \leqslant \sum_{\mu=1}^{2^{n+2}-1}\left|\frac{\lambda_{\mu}}{\mu^{\rho}}-\frac{\lambda_{\mu+1}}{(\mu+1)^{\rho}}\right|+\left|\frac{\lambda_{2^{n+2}}}{2^{(n+2) \rho}}\right|$ and the fact that $\frac{\omega_{\alpha+\rho}(f, t)}{t^{\alpha+\rho}}$ is almost decreasing (see Lemma 1.2.2). To estimate $\left\|\widetilde{V}_{2^{n}}^{(\alpha+\rho)}\left(f^{\left(\frac{\lambda n}{n^{\rho}}, 0\right)}\right)\right\|_{p}$, we will need the following lemma, which follows applying the Abel transformation.

Lemma 1.7.1. Let $\mu=\left\{\mu_{n}\right\}_{n=1}^{\infty}$. Let

$$
T_{n}(x)=\sum_{\nu=1}^{n}\left(a_{\nu} \cos \nu x+b_{\nu} \sin \nu x\right)
$$

and

$$
T_{n}(\mu, x)=\sum_{\nu=1}^{n} \mu_{\nu}\left(a_{\nu} \cos \nu x+b_{\nu} \sin \nu x\right)
$$

For any $M>N+1, M \in \mathbb{N}, N \in \mathbb{N} \cup\{0\}$, we have

$$
\begin{aligned}
& T_{M}(\mu, x)-T_{n}(\mu, x)=\mu_{M}\left(T_{M}(x)-T_{N}(x)\right) \\
& +\frac{1}{\pi} \int_{-\pi}^{\pi}\left(T_{M}-T_{N}\right)(x+u)\left[\sum_{j=N+1}^{M-2}\left(\mu_{j}-2 \mu_{j+1}+\mu_{j+2}\right)(j+1) K_{j}(u)\right. \\
& \left.+\left(\mu_{M-1}-\mu_{M}\right) M K_{M-1}(u)\right] d u=\mu_{M}\left(T_{M}(x)-T_{N}(x)\right) \\
& +\frac{1}{\pi} \int_{-\pi}^{\pi}\left(T_{M}-T_{N}\right)(x+u)\left[\sum_{j=N+1}^{M-2} \Delta^{2}\left(\mu_{j}\right)(j+1) K_{j}(u)+\Delta\left(\mu_{M-1}\right) M K_{M-1}(u)\right] d u,
\end{aligned}
$$


where

$$
\begin{aligned}
& K_{n}(x)=\frac{1}{n+1} \sum_{\nu=0}^{n}\left(\frac{1}{2}+\sum_{m=1}^{\nu} \cos m x\right)=\frac{1}{n+1} \sum_{\nu=0}^{n} D_{\nu}(x) . \\
& \text { Using Lemma 1.7.1 with } \mu=\left\{\mu_{n}=\frac{\lambda_{n}}{n^{\rho}}\right\}, M=2^{k+2}, N=2^{k} \text {, we have } \\
& \left\|\widetilde{V}_{2^{n}}^{(\alpha+\rho)}\left(f^{\left(\left\{\frac{\lambda n}{n^{\rho}}\right\}, 0\right)}\right)\right\|_{p} \\
& =\left\|\sum_{k=0}^{n-1}\left(\widetilde{V}_{2^{k+1}}^{(\alpha+\rho)}\left(f^{\left(\left\{\frac{\lambda n}{n^{\rho}}\right\}, 0\right)}\right)-\widetilde{V}_{2^{k}}^{(\alpha+\rho)}\left(f^{\left(\left\{\frac{\lambda n}{n^{\rho}}\right\}, 0\right)}\right)\right)+\widetilde{V}_{1}^{(\alpha+\rho)}\left(f^{\left(\left\{\frac{\lambda n}{n^{\rho}}\right\}, 0\right)}\right)\right\|_{p} \\
& =\| \lambda_{1} \widetilde{V}_{1}^{(\alpha+\rho)}(f)(x)+\sum_{k=0}^{n-1}\left[\frac{\lambda_{2^{k+2}}}{2^{(k+2) \rho}}\left(\widetilde{V}_{2^{k+1}}^{(\alpha+\rho)}(f)(x)-\widetilde{V}_{2^{k}}^{(\alpha+\rho)}(f)(x)\right)\right. \\
& +\frac{1}{\pi} \int_{-\pi}^{\pi}\left(\widetilde{V}_{2^{k+1}}^{(\alpha+\rho)}(f)-\widetilde{V}_{2^{k}}^{(\alpha+\rho)}(f)\right)(x+u) \\
& \left.\times\left(\sum_{j=2^{k}+1}^{2^{k+2}-2} \Delta_{2}\left(\frac{\lambda_{j}}{j^{\rho}}\right)(j+1) K_{j}(u)+\Delta\left(\frac{\lambda_{2^{k+2}-1}}{\left(2^{k+2}-1\right)^{\rho}}\right) 2^{k+2} K_{2^{k+2}-1}(u)\right) d u\right] \|_{p} \\
& \leqslant \sum_{k=0}^{n-1}\left|\frac{\lambda_{2^{k+2}}}{2^{(k+2) \rho}}\right|\left\|\widetilde{V}_{2^{k+1}}^{(\alpha+\rho)}(f)(x)-\widetilde{V}_{2^{k}}^{(\alpha+\rho)}(f)(x)\right\|_{p} \\
& +\left\|\lambda_{1} \widetilde{V}_{1}^{(\alpha+\rho)}(f)(x)\right\|_{p}+\sum_{k=0}^{n-1}\left\|\widetilde{V}_{2^{k+1}}^{(\alpha+\rho)}(f)-\widetilde{V}_{2^{k}}^{(\alpha+\rho)}(f)\right\|_{p} \\
& \times\left(\sum_{j=2^{k}+1}^{2^{k+2}-2}\left|\Delta_{2}\left(\frac{\lambda_{j}}{j^{\rho}}\right)\right|(j+1)+\left|\Delta\left(\frac{\lambda_{2^{k+2}-1}}{\left(2^{k+2}-1\right)^{\rho}}\right)\right| 2^{k+2}\right)=: J_{3}+J_{4}+J_{5} .
\end{aligned}
$$

To estimate $J_{3}$, we first note that by Lemma 1.5.1,

$$
\left\|\widetilde{V}_{2^{k+1}}(f)-\widetilde{V}_{2^{k}}(f)\right\|_{p} \lesssim\left\|V_{2^{k+1}}(f)-V_{2^{k}}(f)\right\|_{p} .
$$

Then, making use of Lemma 1.4.1, we get

$$
\begin{aligned}
J_{3} & \lesssim \sum_{k=0}^{n-1} \frac{\lambda_{2^{k+2}}}{2^{(k+2) \rho}}\left\|V_{2^{k+1}}^{(\alpha+\rho)}(f)(x)-V_{2^{k}}^{(\alpha+\rho)}(f)(x)\right\|_{p} \\
& \lesssim \sum_{k=0}^{n-1} \frac{\lambda_{2^{k+2}}}{2^{(k+2) \rho}} 2^{k(\rho+\alpha)} \omega_{\alpha+\rho}\left(f, \frac{1}{2^{k}}\right)_{p}
\end{aligned}
$$


Further, we have

$$
\begin{aligned}
J_{4}+J_{5} \lesssim\left\|\lambda_{1} V_{1}^{(\alpha+\rho)}(f)(x)\right\|_{p}+\sum_{k=0}^{n-1}\left\|\left\{V_{2^{k+1}}^{(\alpha+\rho)}(f)-V_{2^{k}}^{(\alpha+\rho)}(f)\right\}(x)\right\|_{p} \\
\quad \times\left[\sum_{j=2^{k}+1}^{2^{k+2}-2}\left|\Delta_{2}\left(\frac{\lambda_{j}}{j^{\rho}}\right)\right|(j+1)+\left|\Delta\left(\frac{\lambda_{2^{k+2}-1}}{\left(2^{k+2}-1\right)^{\rho}}\right)\right| 2^{k+2}\right] \\
\lesssim\left\|\lambda_{1} V_{1}^{(\alpha+\rho)}(f)(x)\right\|_{p}+\sum_{k=0}^{n-1}\left\|\left\{V_{2^{k+1}}^{(\alpha+\rho)}(f)-V_{2^{k}}^{(\alpha+\rho)}(f)\right\}(x)\right\|_{p} 2^{k}\left|\Delta \frac{\lambda_{2^{k}}}{2^{k \rho}}\right| \\
\lesssim \sum_{\mu=1}^{2^{n-1}}\left|\frac{\lambda_{\mu}}{\mu^{\rho}}-\frac{\lambda_{\mu+1}}{(\mu+1)^{\rho}}\right| \mu^{\alpha+\rho} \omega_{\alpha+\rho}\left(f, \frac{1}{\mu}\right)_{p} .
\end{aligned}
$$

Collecting estimates of $J_{1}, J_{2}, J_{3}, J_{4}$, and $J_{5}$, we obtain that

$$
\begin{aligned}
& \left\|V_{2^{n}}^{(\alpha)}\left(f^{(\lambda, \beta)}\right)\right\|_{p} \lesssim \sum_{\mu=1}^{2^{n+2}-1}\left|\frac{\lambda_{\mu}}{\mu^{\rho}}-\frac{\lambda_{\mu+1}}{(\mu+1)^{\rho}}\right| \mu^{\alpha+\rho} \omega_{\alpha+\rho}\left(f, \frac{1}{\mu}\right)_{p} \\
& +\left|\lambda_{2^{n+2}}\right| 2^{n \alpha} \omega_{\alpha+\rho}\left(f, \frac{1}{2^{n}}\right)_{p}+\left|\sin \frac{\pi(\beta-\rho)}{2}\right| \sum_{k=0}^{n-1}\left|\lambda_{2^{k+2}}\right| 2^{k \alpha} \omega_{\alpha+\rho}\left(f, \frac{1}{2^{k}}\right)_{p}
\end{aligned}
$$

Combining (1.7.47) and (1.7.48) and taking into account Jackson's inequality, we arrive at (1.7.44). The proof of Theorem 1.7.1 is now complete.

\subsection{Estimates from below of best approximations for general- ized Liouville - Weyl derivatives}

Theorem 1.8.1. Let $1<p<\infty, \max (p, 2) \leqslant \tau<\infty$, and $\lambda=\left\{\lambda_{n}\right\}_{n=1}^{\infty}$ be a sequence of positive numbers such that $\lambda \in G M$. Let the sequence $\left\{\lambda_{n}\right\}_{n=1}^{\infty}$ satisfy the additional condition

$$
\left(\sum_{k=1}^{n}\left|\lambda_{2^{k}}^{\tau}-\lambda_{2^{k-1}}^{\tau}\right|\right)^{\frac{1}{\tau}} \leqslant C\left|\lambda_{2^{n}}\right|
$$

for all integer $n$, where the constant $C$ is independent of $n$. 
If for $f \in L_{p}$ there exists a function $f^{(\lambda, \beta)} \in L_{p}$, with the Fourier series $\sigma(f, \lambda, \beta)(\beta \in \mathbb{R})$, then

$$
\left\|f^{(\lambda, \beta)}\right\|_{p} \gtrsim\left(\lambda_{1}^{\tau} E_{0}^{\tau}(f)_{p}+\sum_{\nu=1}^{\infty}\left|\lambda_{2^{\nu}}^{\tau}-\lambda_{2^{\nu-1}}^{\tau}\right| E_{2^{\nu}-1}^{\tau}(f)_{p}\right)^{\frac{1}{\tau}}
$$

and

$$
E_{2^{m}-1}\left(f^{(\lambda, \beta)}\right)_{p} \gtrsim\left(\lambda_{2^{m-1}}^{\tau} E_{2^{m}-1}^{\tau}(f)_{p}+\sum_{\nu=m}^{\infty}\left|\lambda_{2^{\nu}}^{\tau}-\lambda_{2^{\nu-1}}^{\tau}\right| E_{2^{\nu}-1}^{\tau}(f)_{p}\right)^{\frac{1}{\tau}} .
$$

Remark 1.8.1. Note that if $\left\{\lambda_{n}\right\}$ satisfies the condition $\left\{\lambda_{n}^{\tau}\right\} \in G M$, then (1.8.49) follows from the condition

$$
\sum_{k=1}^{n} \lambda_{2^{k}} \lesssim \lambda_{2^{n}}
$$

Indeed

$$
\sum_{k=1}^{n}\left|\lambda_{2^{k}}^{\tau}-\lambda_{2^{k+1}}^{\tau}\right| \leqslant \sum_{k=1}^{n} \sum_{s=2^{k}}^{2^{k+1}-1}\left|\lambda_{s}^{\tau}-\lambda_{s+1}^{\tau}\right| \lesssim \sum_{k=1}^{n} \lambda_{2^{k}}^{\tau} \lesssim \lambda_{2^{n}}^{\tau} .
$$

The last inequality is equivalent to (1.8.50). For more information concerning condition (1.8.50) see [76, 77].

Proof of Theorem 1.8.1. We consider the series

$$
\sum_{n=1}^{\infty} \lambda_{2^{n-1}} \Delta_{n}=\sum_{n=1}^{\infty} \Lambda_{\nu} \lambda_{\nu} A_{\nu}(x)
$$

where $\Lambda_{\nu}=\frac{\lambda_{2} n-1}{\lambda_{\nu}}$ for $2^{n-1} \leqslant \nu \leqslant 2^{n}-1(n=1,2, \ldots)$. Taking into account that $\lambda \in G M$, we have $\left|\lambda_{k}\right| \leqslant C\left|\lambda_{n}\right|$ for $n \leqslant k \leqslant 2 n$.

We will show that the following inequality follows from (1.8.49)

$$
C_{1} \lambda_{s} \leqslant \lambda_{k} \leqslant C_{2} \lambda_{s}, \quad k \leqslant s \leqslant 2 k .
$$

The left-hand side estimate follows from the $G M$ condition. To prove the right-hand side, let $2^{n} \leqslant k<2^{n+1}$. Then $2^{n+1} \leqslant 2 k<2^{n+2}$ and

$$
\begin{aligned}
\lambda_{k}^{\tau} & \leqslant C \lambda_{2^{n}}^{\tau}=C\left(\lambda_{2^{n+2}}^{\tau}+\sum_{m=n}^{n+1}\left(\lambda_{2^{m}}^{\tau}-\lambda_{2^{m+1}}^{\tau}\right)\right) \\
& \leqslant C\left(\lambda_{2^{n+2}}^{\tau}+\sum_{m=1}^{n+2}\left|\lambda_{2^{m}}^{\tau}-\lambda_{2^{m-1}}^{\tau}\right|\right) \leqslant C \lambda_{2^{n+2}}^{\tau} \leqslant C \lambda_{2 k}^{\tau} .
\end{aligned}
$$


Thus, $\lambda_{k} \leqslant C \lambda_{2 k} \leqslant C \lambda_{s}$ and (1.8.52) follows. Let us now show that (1.8.52) implies that $\left\{1 / \lambda_{n}\right\} \in G M$, that is,

$$
\sum_{k=n}^{2 n}\left|\frac{1}{\lambda_{k}}-\frac{1}{\lambda_{k+1}}\right| \leqslant \frac{C}{\lambda_{n}}
$$

Indeed,

$$
C \lambda_{n} \geqslant \sum_{k=n}^{2 n}\left|\lambda_{k}-\lambda_{k+1}\right|=\sum_{k=n}^{2 n} \lambda_{k} \lambda_{k+1}\left|\frac{1}{\lambda_{k}}-\frac{1}{\lambda_{k+1}}\right| \geqslant C \lambda_{n}^{2} \sum_{k=n}^{2 n}\left|\frac{1}{\lambda_{k}}-\frac{1}{\lambda_{k+1}}\right| .
$$

We also note that

1) $\left|\Lambda_{\nu}\right|=\left|\frac{\lambda_{2^{n-1}}}{\lambda_{\nu}}\right| \leqslant C\left|\frac{\lambda_{2^{n-1}}}{\lambda_{2^{n-1}}}\right| \leqslant M$,

2) for $n=1$,

$$
\sum_{\nu=2^{n-1}}^{2^{n}-1}\left|\Lambda_{\nu}-\Lambda_{\nu+1}\right|=\left|\frac{\lambda_{1}}{\lambda_{1}}-\frac{\lambda_{2}}{\lambda_{2}}\right| \leqslant M,
$$

3) for $n=2,3, \ldots$,

$$
\begin{aligned}
& \sum_{\nu=2^{n-1}}^{2^{n}-1}\left|\Lambda_{\nu}-\Lambda_{\nu+1}\right|=\sum_{\nu=2^{n-1}}^{2^{n}-2}\left|\Lambda_{\nu}-\Lambda_{\nu+1}\right|+\left|\Lambda_{2^{n}-1}-\Lambda_{2^{n}}\right| \\
& =\lambda_{2^{n-1}} \sum_{\nu_{1}=2^{n-1}}^{2^{n}-2}\left|\frac{1}{\lambda_{\nu}}-\frac{1}{\lambda_{\nu+1}}\right|+\left|\frac{\lambda_{2^{n-1}}}{\lambda_{2^{n}-1}}-\frac{\lambda_{2^{n}}}{\lambda_{2^{n}}}\right| \leqslant M .
\end{aligned}
$$

Since the sequence $\left\{\Lambda_{n}\right\}_{n=1}^{\infty}$ satisfies the conditions of Lemma 1.5.3, then the series (1.8.51) is the Fourier series of a function $g(x) \in L_{p}$, and $\|g\|_{p} \leqslant$ $C(p)\left\|f^{(\lambda, \beta)}\right\|_{p}$.

Applying Lemmas 1.5.6 and 1.5.8, we have

$$
\begin{aligned}
I: & =\lambda_{1}^{\tau} E_{0}^{\tau}(f)_{p}+\sum_{\nu=1}^{\infty}\left|\lambda_{2^{\nu}}^{\tau}-\lambda_{2^{\nu-1}}^{\tau}\right| E_{2^{\nu}-1}^{\tau}(f)_{p} \\
& \lesssim \lambda_{1}^{\tau} E_{0}^{\tau}(f)_{p}+\sum_{\nu=1}^{\infty}\left|\lambda_{2^{\nu}}^{\tau}-\lambda_{2^{\nu-1}}^{\tau}\right|\left(\int_{0}^{2 \pi}\left[\sum_{n=\nu+1}^{\infty} \Delta_{n}^{2}\right]^{\frac{p}{2}} d x\right)^{\frac{\tau}{p}} \\
& \lesssim \lambda_{1}^{\tau} E_{0}^{\tau}(f)_{p}+\left(\int_{0}^{2 \pi}\left\{\sum_{\nu=1}^{\infty}\left|\lambda_{2^{\nu}}^{\tau}-\lambda_{2^{\nu-1}}^{\tau}\right|\left[\sum_{n=\nu+1}^{\infty} \Delta_{n}^{2}\right]^{\frac{\tau}{2}}\right\}^{\frac{p}{\tau}} d x\right)^{\frac{\tau}{p}}
\end{aligned}
$$




$$
\lesssim \lambda_{1}^{\tau} E_{0}^{\tau}(f)_{p}+\left(\int_{0}^{2 \pi}\left\{\sum_{\nu=1}^{\infty}\left[\sum_{n=\nu}^{\infty}\left|\lambda_{2^{\nu}}^{\tau}-\lambda_{2^{\nu-1}}^{\tau}\right|^{\frac{2}{\tau}} \Delta_{n+1}^{2}\right]^{\frac{\tau}{2}}\right\}^{\frac{p}{\tau}} d x\right)^{\frac{\tau}{p}}
$$

Using condition (1.8.49), Lemma 1.5.5 with $\tau \geqslant 2$ and Lemma 1.5.8, we get

$$
\begin{aligned}
I & \lesssim \lambda_{1}^{\tau} E_{0}^{\tau}(f)_{p}+\left(\int_{0}^{2 \pi}\left\{\sum_{n=1}^{\infty}\left\{\sum_{\nu=1}^{n}\left[\left|\lambda_{2^{\nu}}^{\tau}-\lambda_{2^{\nu-1}}^{\tau}\right|^{\frac{2}{\tau}} \Delta_{n+1}^{2}\right]^{\frac{\tau}{2}}\right\}^{\frac{2}{\tau}}\right\}^{\frac{p}{2}} d x\right)^{\frac{\tau}{p}} \\
& =\lambda_{1}^{\tau} E_{0}^{\tau}(f)_{p}+\left(\int \sum_{0}^{\infty}\left\{\Delta_{n+1}^{2}\left\{\sum_{\nu=1}^{n}\left|\lambda_{2^{\nu}}^{\tau}-\lambda_{2^{\nu-1}}^{\tau}\right|\right\}^{\frac{2}{\tau}}\right\}^{\frac{p}{2}} d x\right)^{\frac{\tau}{p}} \\
& \lesssim \lambda_{1}^{\tau} E_{0}^{\tau}(f)_{p}+\left(\int_{0}^{2 \pi}\left\{\sum_{n=1}^{\infty} \Delta_{n+1}^{2} \lambda_{2^{n}}^{2}\right\}^{\frac{p}{2}} d x\right)^{\frac{\tau}{p}} \\
& =\lambda_{1}^{\tau} E_{0}^{\tau}(f)_{p}+\left(\int_{0}^{2 \pi}\left\{\sum_{n=2}^{\infty} \Delta_{n}^{2} \lambda_{2^{n-1}}^{2}\right\}^{\frac{p}{2}} d x\right)^{\frac{\tau}{p}} \\
& \lesssim \lambda_{1}^{\tau}\left(\int_{0}^{2 \pi}\left\{\sum_{n=1}^{\infty} \Delta_{n}^{2}\right\}^{\frac{p}{2}} d x\right)^{\frac{\tau}{p}}+\left(\int_{0}^{2 \pi}\left\{\sum_{n=2}^{\infty} \Delta_{n}^{2} \lambda_{2^{n-1}}^{2}\right\}^{\frac{p}{2}} d x\right)^{\frac{\tau}{p}} \\
& \lesssim \lambda_{1}^{\tau}\left(\int_{0}^{2 \pi}\left\{\Delta_{1}^{2}\right\}^{\frac{p}{2}} d x\right)^{\frac{\tau}{p}}+\lambda_{1}^{\tau}\left(\int_{0}^{2 \pi}\left\{\sum_{n=2}^{\infty} \Delta_{n}^{2}\right\}^{\frac{p}{2}} d x\right)^{\frac{\tau}{p}} \\
& +\left(\int_{0}^{2 \pi}\left\{\sum_{n=2}^{\infty} \Delta_{n}^{2} \lambda_{2^{n-1}}^{2}\right\}^{\frac{p}{2}} d x\right)^{\frac{\tau}{p}} .
\end{aligned}
$$

Now we use the fact that

$$
\lambda_{1}^{\tau} \leqslant \lambda_{2^{n-1}}^{\tau}+\sum_{\nu=1}^{n-1}\left|\lambda_{2^{\nu-1}}^{\tau}-\lambda_{2^{\nu}}^{\tau}\right| \leqslant C \lambda_{2^{n-1}}^{\tau}, \quad n=2,3, \ldots
$$

We arrive at

$$
I \lesssim\left(\int_{0}^{2 \pi}\left[\sum_{n=1}^{\infty} \Delta_{n}^{2} \lambda_{2^{n-1}}^{2}\right]^{\frac{p}{2}} d x\right)^{\frac{\tau}{p}} \lesssim\|g\|_{p}^{\tau} \lesssim\left\|f^{(\lambda, \beta)}\right\|_{p}^{\tau}
$$

Thus, we have shown that 


$$
\left\|f^{(\lambda, \beta)}\right\|_{p} \gtrsim\left(\lambda_{1}^{\tau} E_{0}^{\tau}(f)_{p}+\sum_{\nu=1}^{\infty}\left|\lambda_{2^{\nu}}^{\tau}-\lambda_{2^{\nu-1}}^{\tau}\right| E_{2^{\nu}-1}^{\tau}(f)_{p}\right)^{\frac{1}{\tau}} .
$$

Now we estimate $E_{2^{m}-1}(\varphi)_{p}$ from below as follows:

$$
E_{2^{m}-1}\left(f^{(\lambda, \beta)}\right)_{p} \gtrsim\left\{\int_{0}^{2 \pi}\left[\sum_{k=m}^{\infty} \lambda_{2^{k}}^{2} \Delta_{k}^{2}\right]^{\frac{p}{2}} d x\right\}^{\frac{1}{p}}
$$

Using Lemmas 1.5.6 and 1.5.8, we obtain

$$
\begin{aligned}
J: & =\left(\lambda_{2^{m-1}}^{\tau} E_{2^{m}-1}^{\tau}(f)_{p}+\sum_{\nu=m}^{\infty}\left|\lambda_{2^{\nu}}^{\tau}-\lambda_{2^{\nu-1}}^{\tau}\right| E_{2^{\nu}-1}^{\tau}(f)_{p}\right)^{\frac{1}{\tau}} \\
& \lesssim\left(\lambda_{2^{m-1}}^{\tau}\left(\int_{0}^{2 \pi}\left[\sum_{n=m}^{\infty} \Delta_{n+1}^{2}\right]^{\frac{p}{2}} d x\right)^{\frac{\tau}{p}}\right. \\
& \left.+\sum_{\nu=m}^{\infty}\left|\lambda_{2^{\nu}}^{\tau}-\lambda_{2^{\nu-1}}^{\tau}\right|\left(\int_{0}^{2 \pi}\left[\sum_{n=\nu}^{\infty} \Delta_{n+1}^{2}\right]^{\frac{p}{2}} d x\right)^{\frac{\tau}{p}}\right)^{\frac{1}{\tau}} \\
& \lesssim\left(\lambda_{2^{m}}^{\tau}\left(\int_{0}^{2 \pi}\left[\sum_{n=m}^{\infty} \Delta_{n+1}^{2}\right]^{\frac{p}{2}} d x\right)^{\frac{\tau}{p}}\right. \\
& +\left(\int_{0}^{2 \pi}\left\{\sum_{\nu=m}^{\infty}\left|\lambda_{2^{\nu}}^{\tau}-\lambda_{2^{\nu-1}}^{\tau}\right|\left[\sum_{n=\nu}^{\infty} \Delta_{n+1}^{2}\right]^{\frac{\tau}{2}}\right\}^{\frac{p}{\tau}} d x\right)^{\frac{\tau}{p}} \\
& =\left(\lambda_{2^{m-1}}^{\tau}\left(\int_{0}^{2 \pi}\left[\sum_{n=m}^{\infty} \Delta_{n+1}^{2}\right]^{\frac{p}{2}} d x\right)^{\frac{\tau}{p}}\right. \\
& \left.+\left(\int_{0}^{2 \pi}\left\{\left\{\sum_{\nu=m}^{\infty}\left[\sum_{n=\nu}^{\infty}\left|\lambda_{2^{\nu}}^{\tau}-\lambda_{2^{\nu-1}}^{\tau}\right|^{\frac{2}{\tau}} \Delta_{n+1}^{2}\right]^{\frac{\tau}{2}}\right\}^{\frac{2}{\tau}}\right\}^{\frac{p}{2}} d x\right)^{\frac{\tau}{p}}\right)^{\frac{1}{\tau}} .
\end{aligned}
$$

Further, applying Lemma 1.5.5 with $\tau \geqslant 2$, we have

$$
J \lesssim\left(\lambda_{2^{m-1}}^{\tau}\left(\int_{0}^{2 \pi}\left[\sum_{n=m}^{\infty} \Delta_{n+1}^{2}\right]^{\frac{p}{2}} d x\right)^{\frac{\tau}{p}}\right.
$$




$$
\begin{aligned}
& \left.+\left(\int_{0}^{2 \pi}\left\{\sum_{n=m}^{\infty}\left\{\sum_{\nu=m}^{n}\left[\left|\lambda_{2^{\nu}}^{\tau}-\lambda_{2^{\nu-1}}^{\tau}\right|^{\frac{2}{\tau}} \Delta_{n+1}^{2}\right]^{\frac{\tau}{2}}\right\}^{\frac{2}{\tau}}\right\}^{\frac{p}{2}} d x\right)^{\frac{\tau}{p}}\right)^{\frac{1}{\tau}} \\
& =\left(\lambda_{2^{m-1}}^{\tau}\left(\int_{0}^{2 \pi}\left[\sum_{n=m}^{\infty} \Delta_{n+1}^{2}\right]^{\frac{p}{2}} d x\right)^{\frac{\tau}{p}}\right. \\
& \left.+\left(\int_{0}^{2 \pi}\left\{\sum_{n=m}^{\infty} \Delta_{n+1}^{2}\left\{\sum_{\nu=m}^{n}\left|\lambda_{2^{\nu}}^{\tau}-\lambda_{2^{\nu}-1}^{\tau}\right|\right\}^{\frac{2}{\tau}}\right\}^{\frac{p}{2}} d x\right)^{\frac{\tau}{p}}\right)^{\frac{1}{\tau}} \\
& \lesssim\left(\lambda_{2^{m-1}}^{\tau}\left(\int_{0}^{2 \pi}\left[\sum_{n=m}^{\infty} \Delta_{n+1}^{2}\right]^{\frac{p}{2}} d x\right)^{\frac{\tau}{p}}+\left(\int_{0}^{2 \pi}\left\{\sum_{n=m}^{\infty} \Delta_{n+1}^{2} \lambda_{2^{n}}^{2}\right\}^{\frac{p}{2}} d x\right)^{\frac{\tau}{p}}\right)^{\frac{1}{\tau}} \\
& =\left(\lambda_{2^{m-1}}^{\tau}\left(\int_{0}^{2 \pi}\left[\sum_{n=m+1}^{\infty} \Delta_{n}^{2}\right]^{\frac{p}{2}} d x\right)^{\frac{\tau}{p}}+\left(\int_{0}^{\infty}\left\{\sum_{n=m+1}^{\infty} \Delta_{n}^{2} \lambda_{2^{n-1}}^{2}\right\}^{\frac{p}{2}} d x\right)^{\frac{\tau}{p}}\right)^{\frac{1}{\tau}} .
\end{aligned}
$$

Further, taking into account that

$$
\lambda_{2^{m-1}}^{\tau}=\sum_{\nu=m}^{n-1}\left(\lambda_{2^{\nu-1}}^{\tau}-\lambda_{2^{\nu}}^{\tau}\right)+\lambda_{2^{n-1}}^{\tau} \leqslant \sum_{\nu=m}^{n-1}\left|\lambda_{2^{\nu-1}}^{\tau}-\lambda_{2^{\nu}}^{\tau}\right|+\lambda_{2^{n-1}}^{\tau} \leqslant C \lambda_{2^{n-1}}^{\tau},
$$

we derive that

$$
\begin{aligned}
J & \lesssim\left(\int_{0}^{2 \pi}\left\{\sum_{n=m+1}^{\infty} \Delta_{n}^{2} \lambda_{2^{n-1}}^{2}\right\}^{\frac{p}{2}} d x\right)^{\frac{1}{p}} \\
& =\left\{\int_{0}^{2 \pi}\left[\sum_{k=m}^{\infty} \lambda_{2^{k}}^{2} \Delta_{k+1}^{2}\right]^{\frac{p}{2}} d x\right\}^{\frac{1}{p}} \lesssim E_{2^{m}-1}\left(f^{(\lambda, \beta)}\right)_{p} .
\end{aligned}
$$

This gives

$$
\left(\lambda_{2^{m-1}}^{\tau} E_{2^{m}-1}^{\tau}(f)_{p}+\sum_{\nu=m}^{\infty}\left|\lambda_{2^{\nu}}^{\tau}-\lambda_{2^{\nu-1}}^{\tau}\right| E_{2^{\nu}-1}^{\tau}(f)_{p}\right)^{\frac{1}{\tau}} \lesssim E_{2^{m}-1}\left(f^{(\lambda, \beta)}\right)_{p},
$$

completing the proof. 


\section{Sharp Ul'yanov inequalities for Liouville-Weyl derivatives. $\left(L_{p}, L_{q}\right)$ inequalities}

In this section, we study sharp $(p, q)$-inequalities of Ul'yanov type for moduli of smoothness of fractional order. In more detail, we obtain estimates of the best approximation and the modulus of smoothness of the generalized Liouville-Weyl derivatives. We give examples showing the sharpness of these inequalities. Main results of this section were published in [31, 33].

2.1 Ul'yanov type inequalities for moduli of smoothness

Ul'yanov [85] proved the following $(p, q)$-inequalities between moduli of continuity

$$
\omega(f, \delta)_{q} \lesssim\left(\int_{0}^{\delta}\left(t^{-\theta} \omega(f, t)_{p}\right)^{q_{1}} \frac{d t}{t}\right)^{1 / q_{1}}
$$

where

$$
1 \leqslant p<q \leqslant \infty, \quad \theta=\frac{1}{p}-\frac{1}{q}
$$

and

$$
q_{1}= \begin{cases}q, & q<\infty \\ 1, & q=\infty\end{cases}
$$

Here $\omega(f, \delta)_{p}=\omega_{1}(f, \delta)_{p}$ is the modulus of continuity and the modulus of smoothness of order $k \in \mathbb{N}$ is given in subsection 1.2 .

The following $(p, q)$ estimate for moduli of smoothness (of an integer order) is due to DeVore, Riemenschneider, and Sharpley [9] and Gol'dman $[20,21]$ :

$$
\omega_{k}(f, \delta)_{q} \lesssim\left(\int_{0}^{\delta}\left(t^{-\theta} \omega_{k}(f, t)_{p}\right)^{q_{1}} \frac{d t}{t}\right)^{1 / q_{1}} .
$$

Similar estimates for moduli of smoothness of a function $f^{(\rho)}$ was proved by Z. Ditzian and S. Tikhonov [11], [12]

$$
\omega_{k}\left(f^{(\rho)}, \delta\right)_{q} \lesssim\left(\int_{0}^{\delta}\left(t^{-\theta-\rho} \omega_{k+\rho}(f, t)_{p}\right)^{q_{1}} \frac{d t}{t}\right)^{1 / q_{1}}
$$


where $\rho \in \mathbb{N} \cup\{0\}$ and $0<p<q \leqslant \infty$.

It is easy to see that (2.1.1) does not give, in general, the sharp estimate. Take, for example, $f \in C^{\infty}$. Then

$$
\omega_{k}\left(f^{(\rho)}, \delta\right)_{q} \asymp \delta^{k} \lesssim\left(\int_{0}^{\delta}\left(t^{-\theta-\rho} t^{k+\rho}\right)^{q} \frac{d t}{t}\right)^{\frac{1}{q}} \asymp \delta^{k-\theta} .
$$

Recently, several authors (see, e.g., [56], [68], [83]) studied the sharp form of Ul'yanov-type inequalities given by

$$
\omega_{\alpha}\left(f^{(\rho)}, \delta\right)_{q} \lesssim\left(\int_{0}^{\delta}\left(t^{-\theta-\rho} \omega_{\alpha+\rho+\theta}(f, t)_{p}\right)^{q} \frac{d t}{t}\right)^{1 / q}
$$

where $\rho>0, \quad 1<p<q<\infty, \quad \theta=\frac{1}{p}-\frac{1}{q}, \quad \alpha>0$, and $f^{(\rho)}$ is $\rho$-fractional derivative in the sense of Weyl of the function $f$. Note that this estimate provides sharper estimate than (2.1.1). See also [10, 19, 34, 45, 49].

S. Tikhonov and W. Trebels [73] investigated the sharp Ul'yanov inequality for the Liouville-Weyl derivatives. Let us recall this definition.

Let the Fourier series of $f \in L_{1}$ be given by

$$
f(x) \sim \sum_{\nu \in \mathbb{Z}} \hat{f}_{\nu} e^{i \nu x}
$$

with $\hat{f}_{\nu}$ being the Fourier coefficients. Then the generalized Liouville-Weyl derivatives $D^{\lambda} f$ can be defined as follows (if the right-hand side is the Fourier series of an integrable function):

$$
D^{\lambda} f \sim \sum_{\nu \in \mathbb{Z}} \lambda_{\nu} \hat{f}_{\nu} e^{i \nu x}, \quad \lambda_{\nu}=\lambda(|\nu|)
$$

where $\lambda:[0, \infty) \rightarrow[0, \infty)$ is an increasing (non-decreasing) function satisfying the following conditions:

$\left(A_{1}\right)$ there exists some $\rho \geqslant 0$ such that $t^{-\rho} \lambda(t)$ is increasing;

$\left(A_{1}^{\prime}\right)$ there is slowly varying, increasing function $\xi:[1, \infty) \rightarrow$ $[c, \infty), c>0$, such that $t^{-\rho} \lambda(t) \xi(t)$ is increasing for some $\rho \geqslant 0$;

$\left(A_{2}\right)$ there exists some $\sigma>0$ such that, with $\rho$ from $\left(A_{1}\right), t^{-\rho-\sigma} \lambda(t)$ is decreasing (i.e. non-increasing);

$\left(A_{3}\right)$ there exists some $\varepsilon>0$ such that, $t^{-\rho-\varepsilon} \lambda(t)$ is increasing (where $\rho \geqslant 0)$ 
$\left(A_{4}\right) \lambda$ is convex or $\lambda^{\prime}$ is locally absolutely continuous and $t\left|\lambda^{\prime \prime}(t)\right| \lesssim$ $\lambda^{\prime}(t), \quad t>0$.

The following three theorems provide the sharp Ul'yanov inequalities for the Liouville-Weyl derivatives in the cases $1<p<q<\infty$ and $p=1<$ $q<\infty$.

Theorem B1. [73] Let $f \in L_{p}, 1<p<q<\infty, \theta=1 / p-1 / q$. Let $\lambda$ satisfy conditions $\left(A_{1}\right)$ and $\left(A_{2}\right)$. Then, for any $\alpha>0$,

$$
\omega_{\alpha}\left(D^{\lambda} f, \delta\right)_{q} \lesssim\left(\int_{0}^{\delta}\left(t^{-\theta} \lambda\left(\frac{1}{t}\right) \omega_{\alpha+\rho+\theta}(f, t)_{p}\right)^{q} \frac{d t}{t}\right)^{1 / q}, \quad 0<\delta<1 .
$$

Theorem B2. [73] Under the hypotheses of theorem B1 with condition $\left(A_{1}\right)$ being replaced by $\left(A_{1}^{\prime}\right)$, we obtain

$\omega_{\alpha}\left(D^{\lambda} f, \delta\right)_{q} \lesssim\left(\int_{0}^{\delta}\left(t^{-\theta} \lambda\left(\frac{1}{t}\right) \xi\left(\frac{1}{t}\right) \omega_{\alpha+\rho+\theta}(f, t)_{p}\right)^{q} \frac{d t}{t}\right)^{1 / q} \quad, \quad \alpha>0, \quad 0<\delta<1$.

Theorem B3. [73] Let $f \in L_{1}, 1<q<\infty, \theta=1-1 / q$.

(i) If $\lambda$ satisfies conditions $\left(A_{1}\right)-\left(A_{3}\right)$. Then

$$
\omega_{\alpha}\left(D^{\lambda} f, \delta\right)_{q} \lesssim\left(\int_{0}^{\delta}\left(t^{-\theta} \lambda\left(\frac{1}{t}\right) \omega_{\alpha+\rho+\theta}(f, t)_{1}\right)^{q} \frac{d t}{t}\right)^{1 / q}, \quad \alpha>0, \quad 0<\delta<1 .
$$

(ii) If $\lambda$ satisfies conditions $\left(A_{1}\right),\left(A_{2}\right)$, and $\left(A_{4}\right)$, then

$\omega_{\alpha}\left(D^{\lambda} f, \delta\right)_{q} \lesssim\left(\int_{0}^{\delta}\left(t^{-\theta} \lambda\left(\frac{1}{t}\right) \omega_{\alpha+\rho+\theta}(f, t)_{1}\right)^{q} \frac{|\ln t| d t}{t}\right)^{1 / q}, \quad \alpha>0, \quad 0<\delta<\frac{1}{2}$

To the best of our knowledge, the Ulyanov type inequalities for the generalized Liouville-Weyl derivatives were not studied in the case $p=1$ and $q=\infty$. However, the following theorem holds for the classical Weyl derivatives.

Theorem B4. Let $f \in L_{1}, \theta=1$, and $\rho \geqslant 0$. We have

$$
\omega_{\alpha}\left(f^{(\rho)}, \delta\right)_{\infty} \lesssim \int_{0}^{\delta} t^{-\rho-\theta} \omega_{\alpha+\rho+\theta}(f, t)_{1} \frac{d t}{t}, \quad \alpha>0, \quad 0<\delta<1
$$


Theorem B4 immediately follows from the sharp Ulyanov inequality ([68])

$$
\omega_{\alpha}(f, \delta)_{\infty} \lesssim \int_{0}^{\delta} t^{-\theta} \omega_{\alpha+1}(f, t)_{1} \frac{d t}{t}, \quad \alpha>0, \quad 0<\delta<1,
$$

and the following $(p, p)$ estimate $(1 \leqslant p \leqslant \infty)$ for the Weyl derivatives ([53], [55, Cor. 2], for a simple proof see also [71, Lemma 2.12]):

$$
\omega_{\alpha}\left(f^{(\rho)}, \delta\right)_{p} \lesssim \int_{0}^{\delta} t^{-\rho} \omega_{\alpha+\rho}(f, t)_{p} \frac{d t}{t}, \quad \alpha, \rho>0, \quad 0<\delta<1 .
$$

Our main objectives are two-fold. The first goal is to prove analogues of Theorems B1, B2, B3, and B4 by considering a more general class of sequences $\left\{\lambda_{n}\right\}$. We replace the monotonicity condition on $\left\{\lambda_{n}\right\}$ by the general monotonicity. Our second goal is to study in detail all limiting cases:

(i) $p=1<q<\infty$;

(ii) $1<p<q=\infty$;

(iii) $p=1<q=\infty$.

\subsection{Inequalities of different metrics for the best approxima- tions}

The following inequality establishes the relationship between the best approximations of a function in different $L_{p}$ metrics. It was proved by Konyushkov and Stechkin (see [37, Theorem 2]). One has

$$
E_{k}(f)_{q} \lesssim k^{\theta} E_{k}(f)_{p}+\sum_{m=k}^{\infty} m^{\theta-1} E_{m}(f)_{p},
$$

where $1 \leqslant p<q \leqslant \infty$ and $\theta=\frac{1}{p}-\frac{1}{q}$.

Inequality (2.2.2) was extended by Ul'yanov [86] for the case of $1 \leqslant p<q<$ $\infty$ as follows:

$$
E_{k}(f)_{q} \lesssim k^{\theta} E_{k}(f)_{p}+\left(\sum_{m=k}^{\infty} m^{q \theta-1} E_{m}^{q}(f)_{p},\right)^{1 / q}, 1 \leqslant p<q<\infty .
$$

Later, Timan [82] obtained the following inequality for the best approximations of $f^{(r)}$ :

$E_{k}\left(f^{(r)}\right)_{q} \lesssim k^{r+\theta} E_{k}(f)_{p}+\left(\sum_{m=k}^{\infty} m^{r q+q \theta-1} E_{m}^{q}(f)_{p},\right)^{1 / q}, \quad 1 \leqslant p<q<\infty, \quad r \in \mathbb{N}$. 
An estimate for best approximations involving generalized derivatives was proved by Szalay [64]:

$$
E_{k}\left(f^{(\lambda)}\right)_{q} \lesssim k^{\theta}\left|\lambda_{k}\right| E_{k}(f)_{p}+\sum_{m=k}^{\infty} m^{\theta} \rho_{m} E_{m}(f)_{p},
$$

where $k \in \mathbb{N}$ and $\rho_{m}=\max \left(\left|\lambda_{m}-\lambda_{m+1}\right|, m^{-1}\left|\lambda_{n}\right|\right)$. Here $f^{(\lambda)}$ defined by (1.1.4) with $\beta=0$. It is assumed in [64] that $\left\{\lambda_{n}\right\}_{n=1}^{\infty}$ is monotonically increasing, convex (or concave) sequence.

\subsection{Some necessary lemmas}

To prove our main result we will use several auxiliary results. The first one is the well-known Nikol'skii inequality for trigonometric polynomials.

Lemma 2.3.1. [8] Let $0<p \leqslant q \leqslant \infty, \quad \theta=\frac{1}{p}-\frac{1}{q}$, and

$$
T_{n}(x)=\frac{a_{0}}{2}+\sum_{\nu=1}^{n}\left(a_{\nu} \cos \nu x+b_{\nu} \sin \nu x\right) .
$$

Then

$$
\left\|T_{n}(x)\right\|_{q} \lesssim n^{\theta}\left\|T_{n}(x)\right\|_{p}
$$

Lemma 2.3.2. [68] For any $T_{n}(x)=\sum_{\nu=1}^{n}\left(a_{\nu} \cos \nu x+b_{\nu} \sin \nu x\right)$, we have

$$
\left\|T_{n}(x)\right\|_{\infty} \leqslant\left\|\left(T_{n}(x)\right)^{\prime}\right\|_{1} .
$$

We will also need the following lemma which was proved in [12].

Lemma 2.3.3. Let $f \in L_{p}, \quad 0<p<q \leqslant \infty, \theta=\frac{1}{p}-\frac{1}{q}$, and $\psi_{\sigma}$ be a near best trigonometric approximant of $f$, i.e., $\left\|\psi_{\sigma}-f\right\|_{L_{p}} \leqslant C E_{\sigma}(f)_{p}$, where $C$ does not depend on $\sigma$. Then

$$
\left\|\sum_{l=1}^{m}\left(\psi_{\sigma 2^{l}}-\psi_{\sigma 2^{l-1}}\right)\right\|_{q} \lesssim\left(\sum_{l=1}^{m}\left(\left(\sigma 2^{l}\right)^{\theta} E_{\sigma 2^{l-1}}(f)_{p}\right)^{q_{1}}\right)^{1 / q_{1}},
$$

where $q_{1}= \begin{cases}q, & q<\infty \\ 1, & q=\infty\end{cases}$

The following result is the Hardy-Littlewood theorem for function with general monotone Fourier coefficients. 
Lemma 2.3.4. [17, 69] Let $\lambda=\{\lambda\}_{m=1}^{\infty} \in G M$. Let the Fourier series of $f$ be given by $\sum_{k=1}^{\infty} \lambda_{k} \cos k x$. A sufficient condition that the function $f$ should belong to $L_{p}, \quad 1<p<\infty$, is that $\sum_{k=1}^{\infty}\left|\lambda_{k}\right|^{p} k^{p-2}<\infty$. Moreover,

$$
\|f\|_{p} \lesssim\left(\sum_{k=1}^{\infty}\left|\lambda_{k}\right|^{p} k^{p-2}\right)^{\frac{1}{p}}
$$

If, additionally, $\left\{\lambda_{n}\right\}_{n=1}^{\infty}$ is a non-negative sequences, then

$$
\|f\|_{p} \asymp\left(\sum_{k=1}^{\infty} \lambda_{k}^{p} k^{p-2}\right)^{\frac{1}{p}} .
$$

\subsection{New results in the case $1<p<q<\infty$}

For this non-limiting case we obtain the following generalization of Theorems B1 and B2.

Theorem 2.4.1. Let $f \in L_{p}, 1<p<q<\infty, \theta=1 / p-1 / q$. Let $\lambda=$ $\left\{\lambda_{n}\right\}_{n=1}^{\infty} \in G M$. Then, for any $\alpha>0$,

$$
\omega_{\alpha}\left(f^{(\lambda, \beta)}, \frac{1}{2^{n}}\right)_{q} \lesssim\left(\sum_{m=n}^{\infty}\left(2^{m(\theta+\rho)} \Lambda_{2^{m}} \omega_{\alpha+\rho+\theta}\left(f, \frac{1}{2^{m}}\right)_{p}\right)^{q}\right)^{1 / q}
$$

where

$$
\Lambda_{n}:=\max _{1 \leqslant k \leqslant n} \frac{\left|\lambda_{2^{k}}\right|}{2^{k \rho}}, \quad \rho>0 .
$$

Remark 2.4.1. Note that if for $f \in L_{p}$ the series

$$
\sum_{m=1}^{\infty}\left(2^{m(\theta+\rho)} \Lambda_{2^{m}} \omega_{\alpha+\rho+\theta}\left(f, \frac{1}{2^{m}}\right)_{p}\right)^{q}
$$

converges, then there exists a function $f^{(\lambda, \beta)} \in L_{q}$ with the Fourier series $\sigma(f, \lambda, \beta)$. Similar remarks are valid for all further theorems.

For the sake of convenience, we formulate Ul'yanov's inequalities for sums. However, the corresponding integral form holds as in Theorems B1B4.

Remark 2.4.2. Using the fact that $\omega_{\alpha}(f, \delta)_{p} \asymp \omega_{\alpha}(f, 2 \delta)_{p}$, we note that under conditions of Theorem 2.4.1, one has

$$
\omega_{\alpha}\left(f^{(\lambda, \beta)}, \delta\right)_{q} \lesssim\left(\int_{0}^{\delta}\left(t^{-\theta-\rho} \Lambda_{\left[\frac{1}{t}\right]} \omega_{\alpha+\rho+\theta}(f, t)_{p}\right)^{q} \frac{d t}{t}\right)^{1 / q} \quad, \quad 0<\delta<1 .
$$


Remark 2.4.3. Note that for any $\left\{\lambda_{n}\right\}_{n=1}^{\infty} \in G M$, we have

$$
\max _{1 \leqslant k \leqslant n} \frac{\left|\lambda_{2^{k}}\right|}{2^{k \rho}} \max _{1 \leqslant s \leqslant 2^{n}} \frac{\left|\lambda_{s}\right|}{s^{\rho}} .
$$

This holds from property 1 in Lemma 1.4.1.

Remark 2.4.4. Theorem 2.4.1 generalizes Theorem B1 and B2.

The proof of this remark will be given below.

Example 2.4.1. For $1<p<q<\infty, \theta=1 / p-1 / q$, Theorem 2.4.1 implies that

$$
\omega_{\alpha}\left(f^{(\lambda, \beta)}, \frac{1}{2^{n}}\right)_{q} \lesssim\left(\sum_{m=n}^{\infty}\left(2^{m(\theta+\rho)} m^{\gamma} \omega_{\alpha+\rho+\theta}\left(f, \frac{1}{2^{m}}\right)_{p}\right)^{q}\right)^{1 / q},
$$

where $\lambda_{n}=n^{\rho} \ln ^{\gamma}(n+1), \quad \gamma \geqslant 0$, and

$$
\omega_{\alpha}\left(f^{(\lambda, \beta)}, \frac{1}{2^{n}}\right)_{q} \lesssim\left(\sum_{m=n}^{\infty}\left(2^{m(\theta+\rho)} \omega_{\alpha+\rho+\theta}\left(f, \frac{1}{2^{m}}\right)_{p}\right)^{q}\right)^{1 / q}
$$

where $\lambda_{n}=\frac{\eta^{\rho}}{\ln ^{\gamma}(n+1)}, \quad \gamma \geqslant 0$.

Note that inequality (2.4.3) also follows from Theorem B1.

Remark 2.4.5. Note that inequality (2.4.4), for $\lambda_{n}=\frac{n^{\rho}}{\ln ^{\gamma}(n+1)}, \gamma \geqslant 0$, cannot be improved as follows.

$$
\omega_{\alpha}\left(f^{(\lambda, \beta)}, \frac{1}{2^{n}}\right)_{q} \lesssim\left(\sum_{m=n}^{\infty}\left(\frac{2^{m(\theta+\rho)}}{m^{\gamma}} \omega_{\alpha+\rho+\theta}\left(f, \frac{1}{2^{m}}\right)_{p}\right)^{q}\right)^{1 / q} .
$$

See Example 3.2 in [73].

Proof of Theorem 2.4.1. We start with the realization result (Lemma 1.2.3)

$$
\omega_{\alpha}\left(f^{(\lambda, \beta)}, \frac{1}{2^{n}}\right)_{q} \lesssim\left\|f^{(\lambda, \beta)}-S_{2^{n}}\left(f^{(\lambda, \beta)}\right)\right\|_{q}+2^{-n \alpha}\left\|S_{2^{n}}^{(\alpha)}\left(f^{(\lambda, \beta)}\right)\right\|_{q}=: I_{1}+I_{2} .
$$

First, we estimate $I_{1}$ using Lemma 2.3.3 to obtain

$$
\left\|S_{2^{l}}\left(f^{(\lambda, \beta)}\right)-S_{2^{n}}\left(f^{(\lambda, \beta)}\right)\right\|_{q} \lesssim\left(\sum_{m=n}^{l-1} 2^{m \theta q}\left\|S_{2^{m+1}}\left(f^{(\lambda, \beta)}\right)-S_{2^{m}}\left(f^{(\lambda, \beta)}\right)\right\|_{p}^{q}\right)^{1 / q} .
$$


By the Marcinkiewicz multiplier theorem, the definition of $G M$ sequences, and the fact that $\left|\lambda_{k}\right| \lesssim\left|\lambda_{2^{m}}\right|, 2^{m} \leqslant k<2^{m+1}$, we have

$$
\left\|S_{2^{m+1}}\left(f^{(\lambda, \beta)}\right)-S_{2^{m}}\left(f^{(\lambda, \beta)}\right)\right\|_{p} \lesssim\left|\lambda_{2^{m}}\right|\left\|S_{2^{m+1}}(f)-S_{2^{m}}(f)\right\|_{p} .
$$

Let us denote $\Lambda_{2^{m}}:=\max _{1 \leqslant k \leqslant m} \frac{\left|\lambda_{2 k}\right|}{2^{k \rho}}$. It is clear that $\left|\lambda_{2^{m}}\right| \leqslant 2^{m \rho} \Lambda_{2^{m}}$. Then

$$
\begin{aligned}
\left|\lambda_{2^{m}}\right|\left\|S_{2^{m+1}}(f)-S_{2^{m}}(f)\right\|_{p} & \leqslant 2^{m \rho} \Lambda_{2^{m}}\left\|S_{2^{m+1}}(f)-S_{2^{m}}(f) \pm f\right\|_{p} \\
& \lesssim 2^{m \rho} \Lambda_{2^{m}} E_{2^{m}}(f)_{p} .
\end{aligned}
$$

Applying Jackson's inequality, we obtain

$$
\begin{aligned}
& I_{1}=\left\|f^{(\lambda, \beta)}-S_{2^{n}}\left(f^{(\lambda, \beta)}\right)\right\|_{p} \lesssim\left(\sum_{m=n}^{\infty} 2^{m(\theta+\rho) q} \Lambda_{2^{m}}^{q} E_{2^{m}}^{q}(f)_{p}\right)^{1 / q} \\
& \lesssim\left(\sum_{m=n}^{\infty} 2^{m(\theta+\rho) q} \Lambda_{2^{m}}^{q} \omega_{\alpha+\rho+\theta}\left(f, \frac{1}{2^{m}}\right)_{p}^{q}\right)^{1 / q} .
\end{aligned}
$$

To estimate $I_{2}$, we use the Hardy-Littlewood fractional integration theorem

$$
\left\|S_{2^{n}}^{(\alpha)}\left(f^{(\lambda, \beta)}\right)\right\|_{q} \lesssim\left\|S_{2^{n}}^{(\alpha+\theta)}\left(f^{(\lambda, \beta)}\right)\right\|_{p} .
$$

Nothing that for any $\left\{\lambda_{n}\right\}_{n=1}^{\infty} \in G M$, we have that $\left\{\frac{\lambda_{n}}{n^{\rho}}\right\}_{n=1}^{\infty} \in G M$ for any $\rho \in \mathbb{R}$ (see Lemma 1.4.6), and applying Lemma 1.5.3, we get

$$
\left\|S_{2^{n}}^{(\alpha+\theta)}\left(f^{(\lambda, \beta)}\right)\right\|_{p} \lesssim \max _{1 \leqslant k \leqslant n} \frac{\left|\lambda_{2^{k}}\right|}{2^{k \rho}}\left\|S_{2^{n}}^{(\alpha+\theta+\rho)}(f)\right\|_{p} .
$$

Using Lemma 1.2.3, we obtain

$$
\begin{aligned}
& I_{2} \lesssim 2^{-n \alpha} \max _{1 \leqslant k \leqslant n} \frac{\left|\lambda_{2^{k}}\right|}{2^{k \rho}}\left\|S_{2^{n}}^{(\alpha+\theta+\rho)}(f)\right\|_{p} \\
& \lesssim 2^{n(\theta+\rho)} \max _{1 \leqslant k \leqslant n} \frac{\left|\lambda_{2^{k}}\right|}{2^{k \rho}} \omega_{\alpha+\rho+\theta}\left(f, \frac{1}{2^{n}}\right)_{p} \\
& =2^{n(\theta+\rho)} \Lambda_{2^{n} \omega_{\alpha+\rho+\theta}}\left(f, \frac{1}{2^{n}}\right)_{p} .
\end{aligned}
$$

Collecting estimates for $I_{1}$ and $I_{2}$, we have

$$
\omega_{\alpha}\left(f^{(\lambda, \beta)}, \frac{1}{2^{n}}\right)_{q} \lesssim\left(\sum_{m=n}^{\infty}\left(2^{m(\theta+\rho)} \Lambda_{2^{m}} \omega_{\alpha+\rho+\theta}\left(f, \frac{1}{2^{m}}\right)_{p}\right)^{q}\right)^{1 / q}
$$

completing the proof. 
Proof of Remark 2.4.4. We show only a nontrivial part that Theorem 2.4.1 extends Theorem B2. Let conditions $\left(A_{1}^{\prime}\right)$ and $\left(A_{2}\right)$ hold. Then $\lambda_{2 n} \lesssim \lambda_{n}$. Assume that $\xi(t)$ is an increasing slowly varying function. Then the function $t^{-\rho} \xi(t)$ is strictly decreasing for $t$ large enough. Suppose that $\frac{\lambda_{n}}{n^{\rho}} \xi(n)$ is increasing. Then we have that $\left\{\lambda_{n}\right\} \in G M$. Indeed, this follows from Lemma 1.4.2, since the conditions of $\lambda$ imply that $\left\{\frac{\lambda_{n} \xi(n)}{n^{\rho}}\right\} \in G M$ and $\left\{\frac{n^{\rho}}{\xi(n)}\right\} \in G M$. Here we have used an obvious fact that any increasing sequence $b_{n}$ such that $b_{2 n} \lesssim b_{n}$ is general monotone.

Now we remark that

$$
\Lambda_{n}=\max _{1 \leqslant k \leqslant n} \frac{\lambda_{2^{k}}}{2^{k \rho}} \xi\left(2^{k}\right)=\frac{\lambda_{2^{n}}}{2^{n \rho}} \xi\left(2^{n}\right) .
$$

Then Theorem 2.4.1 gives

$$
\begin{aligned}
& \omega_{\alpha}\left(f^{(\lambda, \beta)}, \frac{1}{2^{n}}\right)_{q} \lesssim\left(\sum_{m=n}^{\infty}\left(2^{m(\theta+\rho)} \Lambda_{2^{m}} \omega_{\alpha+\rho+\theta}\left(f, \frac{1}{2^{m}}\right)_{p}\right)^{q}\right)^{1 / q} \\
& =\left(\sum_{m=n}^{\infty}\left(2^{m \theta} \lambda_{2^{m}} \xi\left(2^{m}\right) \omega_{\alpha+\rho+\theta}\left(f, \frac{1}{2^{m}}\right)_{p}\right)^{q}\right)^{1 / q}
\end{aligned}
$$

the latter is equivalent to the statement of Theorem B2.

\subsection{New results in the case $p=1<q<\infty$}

The next results provide the sharp Ul'yanov type inequality for the first limiting case and, in particular, generalize Theorem B3.

Theorem 2.5.1. Let $f \in L_{p}, 1=p<q<\infty, \theta=1-1 / q$. Let $\lambda=$ $\left\{\lambda_{n}\right\}_{n=1}^{\infty} \in G M$. Then, for any $\alpha>0$ and $0<\varepsilon \leqslant \min (\rho, \theta)$,

$$
\omega_{\alpha}\left(f^{(\lambda, \beta)}, \frac{1}{2^{n}}\right)_{q} \lesssim\left(\sum_{m=n}^{\infty}\left(2^{m(\theta+\rho)} \Lambda_{2^{m}} \omega_{\alpha+\rho+\theta-\varepsilon}\left(f, \frac{1}{2^{m}}\right)_{1}\right)^{q}\right)^{1 / q}
$$

where

$$
\Lambda_{2^{n}}:=2^{-n \frac{\varepsilon}{2}} \max _{1 \leqslant k \leqslant n} \frac{\left|\lambda_{2^{k}}\right|}{2^{k\left(\rho-\frac{\varepsilon}{2}\right)}} .
$$

Remark 2.5.1. It is clear that Theorem B3 (i) follows from Theorem 2.5.1. 
Proof of Theorem 2.5.1. We consider the de la Vallée-Poussin polynomials $V_{1}(f):=A_{1}(f, x)$ and

$$
V_{n}(f)(x)=\sum_{m=1}^{n} A_{m}(f, x)+\sum_{m=n+1}^{2 n-1}\left(1-\frac{m-n}{n}\right) A_{m}(f, x)(n \geqslant 2),
$$

where $A_{m}(f, x)$ are given by (1.1.2). We start by applying realization inequality (1.2.9) from Lemma 1.2.3

$$
\omega_{\alpha}\left(f^{(\lambda, \beta)}, \frac{1}{2^{n}}\right)_{q} \lesssim\left\|f^{(\lambda, \beta)}-V_{2^{n}}\left(f^{(\lambda, \beta)}\right)\right\|_{q}+2^{-n \alpha}\left\|V_{2^{n}}^{(\alpha)}\left(f^{(\lambda, \beta)}\right)\right\|_{q}=: I_{1}+I_{2} .
$$

Let $0<\varepsilon \leqslant \min (\rho, \theta)$. To estimate $I_{1}$, we use Lemma 2.3.3

$$
\left\|V_{2^{l}}\left(f^{(\lambda, \beta)}\right)-V_{2^{n}}\left(f^{(\lambda, \beta)}\right)\right\|_{q} \lesssim\left(\sum_{m=n}^{l-1} 2^{m \theta_{0} q}\left\|V_{2^{m+1}}\left(f^{(\lambda, \beta)}\right)-V_{2^{m}}\left(f^{(\lambda, \beta)}\right)\right\|_{p_{0}}^{q}\right)^{1 / q},
$$

where $1<p_{0}<q<\infty$ is chosen such that

$$
\theta_{0}=\frac{1}{p_{0}}-\frac{1}{q}=\theta-\frac{\varepsilon}{2}
$$

By Lemmas 1.4.1 and 1.5.3, we get

$$
\left\|V_{2^{m+1}}\left(f^{(\lambda, \beta)}\right)-V_{2^{m}}\left(f^{(\lambda, \beta)}\right)\right\|_{p_{0}} \lesssim\left|\lambda_{2^{m}}\right||| V_{2^{m+1}}(f)-V_{2^{m}}(f) \|_{p_{0}} .
$$

Denoting

$$
\Lambda_{2^{n}}:=2^{-n \varepsilon / 2} \max _{1 \leqslant k \leqslant n} \frac{\left|\lambda_{2^{k}}\right|}{2^{k(\rho-\varepsilon / 2)}},
$$

we have

$$
\left|\lambda_{2^{m}}\right|\left\|V_{2^{m+1}}(f)-V_{2^{m}}(f)\right\|_{p_{0}} \lesssim 2^{m \rho} \Lambda_{2^{m}}\left\|V_{2^{m+1}}(f)-V_{2^{m}}(f)\right\|_{p_{0}} .
$$

Applying Lemma 2.3.1, we obtain

$$
2^{m \rho} \Lambda_{2^{m}}\left\|V_{2^{m+1}}(f)-V_{2^{m}}(f)\right\|_{p_{0}} \lesssim 2^{m \rho} \Lambda_{2^{m}} 2^{m\left(1-\frac{1}{p_{0}}\right)}\left\|V_{2^{m+1}}(f)-V_{2^{m}}(f)\right\|_{1}=: I_{3} .
$$

It is well known that $V_{n}(f)$ is the $L_{p}$ near-best approximant of $f$, that is,

$$
\left\|f-V_{n}(f)\right\|_{p} \lesssim E_{n}(f)_{p} \equiv \inf _{T \in \mathbb{T}_{n}}\|f-T\|_{p},
$$

where $\mathbb{T}_{n}$ is the set of all trigonometric polynomials of degree at most $n$. Then

$$
I_{3} \lesssim 2^{m\left(\rho+1-\frac{1}{p_{0}}\right)} \Lambda_{2^{m}}\left\|V_{2^{m+1}}(f)-V_{2^{m}}(f) \pm f\right\|_{1} \lesssim 2^{m\left(\rho+1-\frac{1}{p_{0}}\right)} \Lambda_{2^{m}} E_{2^{m}}(f)_{1} .
$$


Using Jackson's inequality implies

$$
\begin{aligned}
& I_{1} \lesssim\left(\sum_{m=n}^{\infty}\left(2^{m\left(\theta_{0}+\rho+\theta-\theta_{0}\right)} \Lambda_{2^{m}} E_{2^{m}}(f)_{1}\right)^{q}\right)^{1 / q} \\
\lesssim & \left(\sum_{m=n}^{\infty}\left(2^{m(\theta+\rho)} \Lambda_{2^{m}} \omega_{\alpha+\rho+\theta-\varepsilon}\left(f, \frac{1}{2^{m}}\right)_{p}\right)^{q}\right)^{1 / q} .
\end{aligned}
$$

Now let us estimate the second term in (2.5.5). By Lemma 1.5.4, we have

$$
\left\|V_{2^{n}}^{(\alpha)}\left(f^{(\lambda, \beta)}\right)\right\|_{q} \lesssim\left\|V_{2^{n}}^{\left(\alpha+\theta_{0}\right)}\left(f^{(\lambda, \beta)}\right)\right\|_{p_{0}} .
$$

Note that if $\left\{\lambda_{n}\right\} \in G M$, then $\left\{n^{\xi} \lambda_{n}\right\} \in G M, \quad \xi \in \mathbb{R}$. Again applying Lemmas 1.4.1 and 1.5.3, we obtain

$$
\begin{aligned}
& I_{2} \lesssim 2^{-n \alpha}\left\|V_{2^{n}}^{\left(\alpha+\theta_{0}\right)}\left(f^{(\lambda, \beta)}\right)\right\|_{p_{0}} \\
& \lesssim 2^{-n \alpha} \max _{1 \leqslant k \leqslant n} \frac{\left|\lambda_{2^{k}}\right|}{2^{k(\rho-\varepsilon / 2)}}\left\|V_{2^{n}}^{\left(\alpha+\theta_{0}+\rho-\varepsilon / 2\right)}(f)\right\|_{p_{0}} \\
& =2^{n(\varepsilon / 2-\alpha)} \Lambda_{2^{n}}\left\|V_{2^{n}}^{\left(\alpha+\theta_{0}+\rho-\varepsilon / 2\right)}(f)\right\|_{p_{0} .} .
\end{aligned}
$$

Therefore,

$$
\begin{aligned}
& I_{2} \lesssim 2^{n(\varepsilon / 2-\alpha)} \Lambda_{2^{n}} 2^{n\left(\theta-\theta_{0}\right)}\left\|V_{2^{n}}^{\left(\alpha+\theta_{0}+\rho-\varepsilon / 2\right)}(f)\right\|_{1} \\
& \lesssim 2^{n(\varepsilon / 2-\alpha)} \Lambda_{2^{n}} 2^{n\left(\theta-\theta_{0}\right)} 2^{n\left(\alpha+\theta_{0}+\rho-\varepsilon / 2\right)} \omega_{\alpha+\rho+\theta-\varepsilon / 2-\varepsilon / 2}\left(f, \frac{1}{2^{n}}\right)_{1} \\
& =2^{n(\theta+\rho)} \Lambda_{2^{n}} \omega_{\alpha+\rho+\theta-\varepsilon}\left(f, \frac{1}{2^{n}}\right)_{1} .
\end{aligned}
$$

Here, the first inequality follows from Nikol'skii's inequality given by Lemma 2.3.1 and the second from Lemma 1.5.2. Collecting estimates for $I_{1}$ and $I_{2}$, we obtain

$$
\omega_{\alpha}\left(f^{(\lambda, \beta)}, \frac{1}{2^{n}}\right)_{q} \lesssim\left(\sum_{m=n}^{\infty}\left(2^{m(\theta+\rho)} \Lambda_{2^{m}} \omega_{\alpha+\rho+\theta-\varepsilon}\left(f, \frac{1}{2^{m}}\right)_{1}\right)^{q}\right)^{1 / q},
$$

which completes the proof.

In the next result, unlike Theorem 2.5.1, we deal with $\omega_{\alpha+\rho+\theta}(f, t)_{1}$ obtaining the sharp Ul'yanov inequality. 
Theorem 2.5.2. Let $f \in L_{p}, 1=p<q<\infty, \theta=1-1 / q$, and $\lambda=$ $\left\{\lambda_{n}\right\}_{n=1}^{\infty} \in G M$. Then, for any $\alpha>0$,

$$
\omega_{\alpha}\left(f^{(\lambda, \beta)}, \frac{1}{2^{n}}\right)_{q} \lesssim\left(\sum_{m=n}^{\infty}\left(2^{m(\theta+\rho)} \Lambda_{2^{m}} \omega_{\alpha+\rho+\theta}\left(f, \frac{1}{2^{m}}\right)_{1}\right)^{q}\right)^{1 / q}
$$

where

$$
\Lambda_{N}:=\left(\sum_{k=1}^{N} \frac{\left|\lambda_{k}\right|^{q}}{k^{(q \rho+1)}}\right)^{\frac{1}{q}}, \quad \rho>0 .
$$

Remark 2.5.2. Since the conditions $\left(A_{1}\right),\left(A_{2}\right)$, and $\left(A_{4}\right)$ imply that $\left\{\lambda_{n}=\right.$ $\lambda(n)\} \in G M$ and

$$
\Lambda_{2^{m}}=\left(\sum_{k=1}^{2^{m}}\left(\frac{\left|\lambda_{k}\right|}{k^{\rho}}\right)^{q} \frac{1}{k}\right)^{\frac{1}{q}} \lesssim \frac{\left|\lambda_{2^{m}}\right|}{2^{m \rho}} m^{\frac{1}{q}},
$$

then Theorem 2.5.2 sharpens Theorem B3 (ii).

Example 2.5.1 and Remark 2.5.3 below show that the inequalities in Theorems 2.5.1 and 2.5.2 are not comparable.

Example 2.5.1. Let $1=p<q<\infty, \theta=1-1 / q, \quad \rho>0, \gamma \in \mathbb{R}$, and $\lambda_{n}=n^{\rho} \ln ^{\gamma}(n+1)$. By Theorem 2.5.1, we have

$$
\omega_{\alpha}\left(f^{(\lambda, \beta)}, \frac{1}{2^{n}}\right)_{q} \lesssim\left(\sum_{m=n}^{\infty}\left(2^{m(\theta+\rho)} m^{\gamma} \omega_{\alpha+\rho+\theta-\varepsilon}\left(f, \frac{1}{2^{m}}\right)_{1}\right)^{q}\right)^{1 / q},
$$

and, by Theorem 2.5.2, we have

$$
\omega_{\alpha}\left(f^{(\lambda, \beta)}, \frac{1}{2^{n}}\right)_{q} \lesssim \begin{cases}\left(\sum_{m=n}^{\infty}\left(2^{m(\theta+\rho)} m^{\gamma+\frac{1}{q}} \omega_{\alpha+\rho+\theta}\left(f, \frac{1}{2^{m}}\right)_{1}\right)^{q}\right)^{1 / q}, & \gamma>-\frac{1}{q}, \\ \left(\sum_{m=n}^{\infty}\left(2^{m(\theta+\rho)} \omega_{\alpha+\rho+\theta}\left(f, \frac{1}{2^{m}}\right)_{1}\right)^{q} \ln m\right)^{1 / q}, & \gamma=-\frac{1}{q}, \\ \left(\sum_{m=n}^{\infty}\left(2^{m(\theta+\rho)} \omega_{\alpha+\rho+\theta}\left(f, \frac{1}{2^{m}}\right)_{1}\right)^{q}\right)^{1 / q}, & \gamma<-\frac{1}{q} .\end{cases}
$$

Remark 2.5.3. We note that inequality (2.5.6) for $\gamma \in \mathbb{R}$ and inequality (2.5.7) for $\gamma \geqslant 0$ also follows from Theorem B3 (see [73, Corollary 1.5]). Using $\left\{\Lambda_{n}\right\}$ allows us to prove (2.5.7) for any choice of $\gamma \in \mathbb{R}$. Moreover, in [73] it was shown that estimates (2.5.6) and (2.5.7) are not comparable. 
Proof of Theorem 2.5.2. The proof follows the same lines as those in Theorem 2.5.1. We consider the de la Vallée-Poussin sum of the function $f^{(\lambda, \beta)}$. As above, we use Lemma 1.2.3

$$
\omega_{\alpha}\left(f^{(\lambda, \beta)}, \frac{1}{2^{n}}\right)_{q} \lesssim\left\|f^{(\lambda, \beta)}-V_{2^{n}}\left(f^{(\lambda, \beta)}\right)\right\|_{q}+2^{-n \alpha}\left\|V_{2^{n}}^{(\alpha)}\left(f^{(\lambda, \beta)}\right)\right\|_{q}=: I_{1}+I_{2} .
$$

Now we apply the following $(p, q)$-inequality:

$$
\left\|V_{2^{l}}\left(f^{(\lambda, \beta)}\right)-V_{2^{n}}\left(f^{(\lambda, \beta)}\right)\right\|_{q} \lesssim\left(\sum_{m=n}^{l-1} 2^{m \theta_{0} q}\left\|V_{2^{m+1}}\left(f^{(\lambda, \beta)}\right)-V_{2^{m}}\left(f^{(\lambda, \beta)}\right)\right\|_{p_{0}}^{q}\right)^{1 / q},
$$

where $1<p_{0}<q<\infty, \quad \theta_{0}=\frac{1}{p_{0}}-\frac{1}{q}$. Further,

$$
\left\|V_{2^{m+1}}\left(f^{(\lambda, \beta)}\right)-V_{2^{m}}\left(f^{(\lambda, \beta)}\right)\right\|_{p_{0}} \lesssim\left|\lambda_{2^{m}}\right||| V_{2^{m+1}}(f)-V_{2^{m}}(f) \|_{p_{0}} .
$$

Set

$$
\Lambda_{2^{m}}:=\left(\sum_{k=1}^{2^{m}} \frac{\left|\lambda_{k}\right|^{q}}{k^{q \rho+1}}\right)^{\frac{1}{q}} .
$$

By Lemma 1.4.7, we have $\lambda_{2^{m}} \lesssim 2^{m \rho} \Lambda_{2^{m}}$. Then

$$
\left|\lambda_{2^{m}}\right|\left\|V_{2^{m+1}}(f)-V_{2^{m}}(f)\right\|_{p_{0}} \lesssim 2^{m \rho} \Lambda_{2^{m}}\left\|V_{2^{m+1}}(f)-V_{2^{m}}(f)\right\|_{p_{0}} .
$$

Using Lemma 2.3.1, we get

$$
2^{m \rho} \Lambda_{2^{m}}\left\|V_{2^{m+1}}(f)-V_{2^{m}}(f)\right\|_{p_{0}} \lesssim 2^{m \rho} \Lambda_{2^{m}} 2^{m\left(1-\frac{1}{p_{0}}\right)}\left\|V_{2^{m+1}}(f)-V_{2^{m}}(f)\right\|_{1}=: I_{3} .
$$

Since $V_{n}$ is the $L_{p}$ near-best approximant of $f$, we obtain that

$$
I_{3} \lesssim 2^{m\left(\rho+1-\frac{1}{p_{0}}\right)} \Lambda_{2^{m}}\left\|V_{2^{m+1}}(f)-V_{2^{m}}(f) \pm f\right\|_{1} \lesssim 2^{m\left(\rho+1-\frac{1}{p_{0}}\right)} \Lambda_{2^{m}} E_{2^{m}}(f)_{1} .
$$

Combining these estimates and Jackson's inequality, we derive that

$$
\begin{aligned}
& I_{1} \lesssim\left(\sum_{m=n}^{\infty}\left(2^{m\left(\theta_{0}+\rho+\theta-\theta_{0}\right)} \Lambda_{2^{m}} E_{2^{m}}(f)_{1}\right)^{q}\right)^{1 / q} \\
& \lesssim\left(\sum_{m=n}^{\infty}\left(2^{m(\theta+\rho)} \Lambda_{2^{m}} \omega_{\alpha+\rho+\theta}\left(f, \frac{1}{2^{m}}\right)_{1}\right)^{q}\right)^{1 / q} .
\end{aligned}
$$


Let us estimate $I_{2}$. Applying Young's convolution inequality ([88]) we have

$$
\begin{aligned}
\left\|V_{2^{n}}^{(\alpha)}\left(f^{(\lambda, \beta)}\right)\right\|_{q} & \lesssim\left\|\sum_{m=1}^{2^{n}} \lambda_{m} m^{\alpha} A_{m}(f, x)+\sum_{m=2^{n}+1}^{2^{n+1}-1} \lambda_{m}\left(1-\frac{m-2^{n}}{2^{n}}\right) m^{\alpha} A_{m}(f, x)\right\|_{q} \\
= & \|\left(\sum_{m=1}^{2^{n+1}-1} \frac{\lambda_{m}}{m^{\rho+\theta}} \cos m x\right) *\left(\sum_{m=1}^{2^{n}} m^{\alpha+\rho+\theta} A_{m}(f, x)\right. \\
& \left.\quad+\sum_{m=2^{n}+1}^{2^{n+1}-1}\left(1-\frac{m-2^{n}}{2^{n}}\right) m^{\alpha+\rho+\theta} A_{m}(f, x)\right) \|_{q} \\
\leqslant & \left\|\sum_{m=1}^{2^{n+1}-1} \frac{\lambda_{m}}{m^{\rho+\theta}} \cos m x\right\|\left\|V_{2^{n}}^{(\alpha+\rho+\theta)}(f, x)\right\|_{1} .
\end{aligned}
$$

Using the fact that $\left\{\lambda_{n}\right\}_{n=1}^{\infty} \in G M$ implies that $\left\{\frac{\lambda_{n}}{n^{\rho+\theta}}\right\}_{n=1}^{\infty} \in G M$, Lemma 2.3.4 yields

$\left\|\sum_{m=1}^{2^{n+1}-1} \frac{\lambda_{m}}{m^{\rho+\theta}} \cos m x\right\|_{q} \lesssim\left(\sum_{\nu=1}^{2^{n+1}-1}\left(\frac{\left|\lambda_{\nu}\right|}{\nu^{\rho+\theta}}\right)^{q} \nu^{q-2}\right)^{\frac{1}{q}}=\left(\sum_{\nu=1}^{2^{n+1}-1} \frac{\left|\lambda_{\nu}\right|^{q}}{\nu^{q \rho+1}}\right)^{\frac{1}{q}}=\Lambda_{2^{n+1}-1}$.

Taking into account that $\lambda_{n} \lesssim \lambda_{k}, \quad k \leqslant n \leqslant 2 k$, we can estimate $\Lambda_{2^{m+1}-1} \lesssim$ $\Lambda_{2^{m}}$. Finally, Lemma 1.5.2 implies

$$
I_{2} \lesssim 2^{-n \alpha} \Lambda_{2^{n}}\left\|V_{2^{n}}^{(\alpha+\rho+\theta)}(f, x)\right\|_{1} \lesssim 2^{n(\rho+\theta)} \Lambda_{2^{n}} \omega_{\alpha+\rho+\theta}\left(f, \frac{1}{2^{n}}\right) .
$$

Collecting estimates for $I_{1}$ and $I_{2}$, we get

$$
\omega_{\alpha}\left(f^{(\lambda, \beta)}, \frac{1}{2^{n}}\right)_{q} \lesssim\left(\sum_{m=n}^{\infty}\left(2^{m(\theta+\rho)} \Lambda_{2^{m}} \omega_{\alpha+\rho+\theta}\left(f, \frac{1}{2^{m}}\right)_{1}\right)^{q}\right)^{1 / q},
$$

completing the proof.

\subsection{New results in the case $1<p<q=\infty$}

In the following theorems we investigate the Ulyanov-type inequality for another limiting case: $1<p<q=\infty$. As in the previous subsection we obtain two main theorems. 
Theorem 2.6.1. Let $f \in L_{p}, 1<p<q=\infty, \theta=1 / p$, and $\lambda=\left\{\lambda_{n}\right\}_{n=1}^{\infty} \in$ $G M$. Then, for any $\alpha>0$ and $0<\varepsilon \leqslant \min (\rho, \theta)$,

$$
\omega_{\alpha}\left(f^{(\lambda, \beta)}, \frac{1}{2^{n}}\right)_{\infty} \lesssim \sum_{m=n}^{\infty} 2^{m(\theta+\rho)} \Lambda_{2^{m}} \omega_{\alpha+\rho+\theta-\varepsilon}\left(f, \frac{1}{2^{m}}\right)_{p}
$$

where

$$
\Lambda_{2^{n}}:=2^{-n \varepsilon / 2} \max _{1 \leqslant k \leqslant n} \frac{\left|\lambda_{2^{k}}\right|}{2^{k(\rho-\varepsilon / 2)}} .
$$

Proof of Theorem 2.6.1. The proof is the same as that of Theorem 2.5.1. We use Lemma 1.2.3 for $q=\infty$ to get

$$
\omega_{\alpha}\left(f^{(\lambda, \beta)}, \frac{1}{2^{n}}\right)_{\infty} \lesssim\left\|f^{(\lambda, \beta)}-V_{2^{n}}\left(f^{(\lambda, \beta)}\right)\right\|_{\infty}+2^{-n \alpha}\left\|V_{2^{n}}^{(\alpha)}\left(f^{(\lambda, \beta)}\right)\right\|_{\infty}=: I_{1}+I_{2} .
$$

Observing that

$$
\lambda_{n} \lesssim \Lambda_{n}:=2^{-n \varepsilon / 2} \max _{1 \leqslant k \leqslant n} \frac{\left|\lambda_{2^{k}}\right|}{2^{k(\rho-\varepsilon / 2)}},
$$

we use Lemmas 2.3.3 and 1.5.3 to derive

$$
\begin{aligned}
\left\|V_{2^{l}}\left(f^{(\lambda, \beta)}\right)-V_{2^{n}}\left(f^{(\lambda, \beta)}\right)\right\|_{\infty} & \lesssim \sum_{m=n}^{l-1} 2^{m \theta}\left\|V_{2^{m+1}}\left(f^{(\lambda, \beta)}\right)-V_{2^{m}}\left(f^{(\lambda, \beta)}\right)\right\|_{p}, \\
& \lesssim \sum_{m=n}^{l-1} 2^{m \theta}\left|\lambda_{2^{m}}\right|\left\|V_{2^{m+1}}(f)-V_{2^{m}}(f)\right\|_{p} \\
& \lesssim \sum_{m=n}^{l-1} 2^{m \theta} 2^{m \rho} \Lambda_{2^{m}}\left\|V_{2^{m+1}}(f)-V_{2^{m}}(f)\right\|_{p} .
\end{aligned}
$$

Then

$$
I_{1} \lesssim \sum_{m=n}^{\infty} 2^{m(\theta+\rho)} \Lambda_{2^{m}} \omega_{\alpha+\rho+\theta-\varepsilon}\left(f, \frac{1}{2^{m}}\right)_{p}
$$

By Lemmas 2.3.1, 1.5.4, and 1.5.3, we choose $q_{0}$ such that

$$
\theta_{0}=\frac{1}{p}-\frac{1}{q_{0}}=\theta-\frac{\varepsilon}{2}
$$


and we estimate

$$
\begin{aligned}
I_{2}=2^{-n \alpha}\left\|V_{2^{n}}^{(\alpha)}\left(f^{(\lambda, \beta)}\right)\right\|_{\infty} & \lesssim 2^{-n \alpha} 2^{n \frac{1}{q_{0}}}\left\|V_{2^{n}}^{(\alpha)}\left(f^{(\lambda, \beta)}\right)\right\|_{q_{0}} \\
& \lesssim 2^{-n \alpha} 2^{n \frac{1}{q_{0}}}\left\|V_{2^{n}}^{\left(\alpha+\theta_{0}\right)}\left(f^{(\lambda, \beta)}\right)\right\|_{p} \\
& \lesssim 2^{n\left(\frac{1}{q_{0}}-\alpha\right)} \max _{1 \leqslant k \leqslant n} \frac{\left|\lambda_{2^{k}}\right|}{2^{k(\rho-\varepsilon / 2)}}\left\|V_{2^{n}}^{\left(\alpha+\theta_{0}+\rho-\varepsilon / 2\right)}(f)\right\|_{p} \\
& \lesssim 2^{n\left(\varepsilon / 2-\alpha+\frac{1}{q_{0}}\right)} \Lambda_{n} 2^{n\left(\alpha+\theta_{0}+\rho-\varepsilon / 2\right)} \omega_{\alpha+\rho+\theta-\varepsilon / 2-\varepsilon / 2}\left(f, \frac{1}{2^{n}}\right)_{p} \\
& =2^{n(\theta+\rho)} \Lambda_{n} \omega_{\alpha+\rho+\theta-\varepsilon}\left(f, \frac{1}{2^{n}}\right)_{p} .
\end{aligned}
$$

Combining the obtained estimates, we finally arrive at

$$
\omega_{\alpha}\left(f^{(\lambda, \beta)}, \frac{1}{2^{n}}\right)_{q} \lesssim \sum_{m=n}^{\infty} 2^{m(\theta+\rho)} \Lambda_{m} \omega_{\alpha+\rho+\theta-\varepsilon}\left(f, \frac{1}{2^{m}}\right)_{p} .
$$

The next result is the sharp Ul'yanov inequality for $1<p<q=\infty$.

Theorem 2.6.2. Let $f \in L_{p}, 1<p<q=\infty, \theta=1 / p$, and $\lambda=\left\{\lambda_{n}\right\}_{n=1}^{\infty} \in$ GM. Then, for any $\alpha>0$,

$$
\omega_{\alpha}\left(f^{(\lambda, \beta)}, \frac{1}{2^{n}}\right)_{\infty} \lesssim \sum_{m=n}^{\infty} 2^{m(\theta+\rho)} \Lambda_{2^{m}} \omega_{\alpha+\rho+\theta}\left(f, \frac{1}{2^{m}}\right)_{p},
$$

where

$$
\Lambda_{N}:=\left(\sum_{k=1}^{N} \frac{\left|\lambda_{k}\right|^{p^{\prime}}}{k^{p^{\prime} \rho+1}}\right)^{\frac{1}{p^{\prime}}}, \quad \rho>0 .
$$

Example 2.6.1. Let $f \in L_{p}, 1<p<q=\infty, \theta=1 / p, \rho>0, \gamma \in \mathbb{R}$, and $\lambda_{n}=n^{\rho} \ln ^{\gamma}(n+1)$. Then, by Theorem 2.6.1, we have

$$
\omega_{\alpha}\left(f^{(\lambda, \beta)}, \frac{1}{2^{n}}\right)_{\infty} \lesssim \sum_{m=n}^{\infty} 2^{m(\theta+\rho)} m^{\gamma} \omega_{\alpha+\rho+\theta-\varepsilon}\left(f, \frac{1}{2^{m}}\right)_{p} .
$$

Theorem 2.6.2 yields

$$
\omega_{\alpha}\left(f^{(\lambda, \beta)}, \frac{1}{2^{n}}\right)_{\infty} \lesssim \begin{cases}\sum_{m=n}^{\infty} 2^{m(\theta+\rho)} m^{\gamma+\frac{1}{p^{\prime}}} \omega_{\alpha+\rho+\theta}\left(f, \frac{1}{2^{m}}\right)_{p}, & \gamma>-\frac{1}{p^{\prime}} \\ \sum_{m=n}^{\infty} 2^{m(\theta+\rho)}(\ln m)^{\frac{1}{p^{\prime}}} \omega_{\alpha+\rho+\theta}\left(f, \frac{1}{2^{m}}\right)_{p}, & \gamma=-\frac{1}{p^{\prime}} \\ \sum_{m=n}^{\infty} 2^{m(\theta+\rho)} \omega_{\alpha+\rho+\theta}\left(f, \frac{1}{2^{m}}\right)_{p}, & \gamma<-\frac{1}{p^{\prime}}\end{cases}
$$


Remark 2.6.1. Note that letting formally $\rho=\gamma=0$ in (2.5.7) and (2.6.8) gives

$$
\omega_{\alpha}\left(f, \frac{1}{2^{n}}\right)_{q} \lesssim\left(\sum_{m=n}^{\infty}\left(2^{m \theta} m^{\max \left(\frac{1}{q}, \frac{1}{p^{\prime}}\right)} \omega_{\alpha+\theta}\left(f, \frac{1}{2^{m}}\right)_{p}\right)^{q_{1}}\right)^{\frac{1}{q_{1}}}
$$

for $1<p<q=\infty$ or $1=p<q<\infty, q_{1}=\left\{\begin{array}{ll}q, & q<\infty, \\ 1, & q=\infty,\end{array}\right.$ which is the the sharp Ul'yanov inequality proved in [68].

Proof of Theorem 2.6.2. Let $1<p<q=\infty$. Applying Lemma 1.2.3, we get

$$
\omega_{\alpha}\left(f^{(\lambda, \beta)}, \frac{1}{2^{n}}\right)_{\infty} \lesssim\left\|f^{(\lambda, \beta)}-V_{2^{n}}\left(f^{(\lambda, \beta)}\right)\right\|_{\infty}+2^{-n \alpha}\left\|V_{2^{n}}^{(\alpha)}\left(f^{(\lambda, \beta)}\right)\right\|_{\infty}=: I_{1}+I_{2},
$$

where $V_{2^{n}}\left(f^{(\lambda, \beta)}\right)$ is the de la Vallée-Poussin sum of the function $f^{(\lambda, \beta)}$. As in the proof of Theorem 2.6.1, we have

$$
\begin{aligned}
& \left\|V_{2^{l}}\left(f^{(\lambda, \beta)}\right)-V_{2^{n}}\left(f^{(\lambda, \beta)}\right)\right\|_{\infty} \lesssim \sum_{m=n}^{l-1} 2^{m \theta}\left\|V_{2^{m+1}}\left(f^{(\lambda, \beta)}\right)-V_{2^{m}}\left(f^{(\lambda, \beta)}\right)\right\|_{p} \\
& \lesssim \sum_{m=n}^{l-1} 2^{m \theta}\left|\lambda_{2^{m}}\right|\left\|V_{2^{m+1}}(f)-V_{2^{m}}(f)\right\|_{p} \\
& \lesssim \sum_{m=n}^{l-1} 2^{m(\theta+\rho)} \Lambda_{2^{m}}\left\|V_{2^{m+1}}(f)-V_{2^{m}}(f)\right\|_{p},
\end{aligned}
$$

where $\Lambda_{2^{m}}:=\left(\sum_{k=1}^{2^{m}} \frac{\left|\lambda_{k}\right|^{p^{\prime}}}{k^{p^{\prime} \rho+1}}\right)^{\frac{1}{p^{\prime}}}$ and the last inequality follows from Lemma 1.4.7. We know that $V_{n}(f)$ is the $L_{p}$ near-best approximant of $f$. By Jackson's inequality, we get

$$
\begin{aligned}
& I_{1} \lesssim \sum_{m=n}^{\infty} 2^{m(\theta+\rho)} \Lambda_{2^{m}} E_{2^{m}}(f)_{p} \\
& \lesssim \sum_{m=n}^{\infty} 2^{m(\theta+\rho)} \Lambda_{2^{m}} \omega_{\alpha+\rho+\theta}\left(f, \frac{1}{2^{m}}\right)_{p} .
\end{aligned}
$$

We handle $I_{2}$ similarly. Applying Young's convolution inequality and Lemma 2.3.4, we have

$$
\begin{aligned}
\left\|V_{2^{n}}^{(\alpha)}\left(f^{(\lambda, \beta)}\right)\right\|_{\infty} & \lesssim\left\|\sum_{m=1}^{2^{n+1}-1} \frac{\lambda_{m}}{m^{\rho+\theta}} \cos m x\right\|_{p^{\prime}}\left\|V_{2^{n}}^{(\alpha+\rho+\theta)}(f, x)\right\|_{p} \\
& \lesssim \Lambda_{2^{n+1}}\left\|V_{2^{n}}^{(\alpha+\rho+\theta)}(f, x)\right\|_{p} .
\end{aligned}
$$


Remark that $\Lambda_{2^{n+1}} \lesssim \Lambda_{2^{n}}$. Finally, by Lemma 1.5.2, we obtain

$$
I_{2} \lesssim 2^{-n \alpha} \Lambda_{2^{n}}\left\|V_{2^{n}}^{(\alpha+\rho+\theta)}(f, x)\right\|_{p} \lesssim 2^{n(\rho+\theta)} \Lambda_{2^{n}} \omega_{\alpha+\rho+\theta}\left(f, \frac{1}{2^{n}}\right)_{p} .
$$

Collecting estimates for $I_{1}$ and $I_{2}$, we derive

$$
\omega_{\alpha}\left(f^{(\lambda, \beta)}, \frac{1}{2^{n}}\right)_{q} \lesssim \sum_{m=n}^{\infty} 2^{m(\theta+\rho)} \Lambda_{2^{m}} \omega_{\alpha+\rho+\theta}\left(f, \frac{1}{2^{m}}\right)_{p} .
$$

\subsection{New results in the case $1=p, q=\infty$}

Our two main results here are Theorem 2.7.1 and 2.7.2 below.

Theorem 2.7.1. Let $f \in L_{p}, p=1, q=\infty, \theta=1$, and $\lambda=\left\{\lambda_{n}\right\}_{n=1}^{\infty} \in G M$. Then, for any $\alpha>0$ and $0<\varepsilon \leqslant \min (\rho, \theta)$,

$$
\omega_{\alpha}\left(f^{(\lambda, \beta)}, \frac{1}{2^{n}}\right)_{\infty} \lesssim \sum_{m=n}^{\infty} 2^{m(\theta+\rho)} \Lambda_{2^{m}} \omega_{\alpha+\rho+\theta-\varepsilon}\left(f, \frac{1}{2^{m}}\right)_{1}
$$

where

$$
\Lambda_{2^{n}}:=2^{-n \varepsilon / 2} \max _{1 \leqslant k \leqslant n} \frac{\left|\lambda_{2^{k}}\right|}{2^{k(\rho-\varepsilon / 2)}} .
$$

Proof of Theorem 2.7.1. Let $p=1<q=\infty$. The method of proof is the same as above. Lemma 1.2.3 with $q=\infty$ gives

$$
\omega_{\alpha}\left(f^{(\lambda, \beta)}, \frac{1}{2^{n}}\right)_{\infty} \lesssim\left\|f^{(\lambda, \beta)}-V_{2^{n}}\left(f^{(\lambda, \beta)}\right)\right\|_{\infty}+2^{-n \alpha}\left\|V_{2^{n}}^{(\alpha)}\left(f^{(\lambda, \beta)}\right)\right\|_{\infty}=: I_{1}+I_{2} .
$$

Applying Lemma 2.3.3 and 1.5.3, we obtain for $\theta_{0}=1 / p_{0}$

$$
\begin{aligned}
& \left\|V_{2^{l}}\left(f^{(\lambda, \beta)}\right)-V_{2^{n}}\left(f^{(\lambda, \beta)}\right)\right\|_{\infty} \lesssim \sum_{m=n}^{l-1} 2^{m \theta_{0}}\left\|V_{2^{m+1}}\left(f^{(\lambda, \beta)}\right)-V_{2^{m}}\left(f^{(\lambda, \beta)}\right)\right\|_{p_{0}} \\
& \lesssim \sum_{m=n}^{l-1} 2^{m \theta_{0}}\left|\lambda_{2^{m}}\right|\left\|V_{2^{m+1}}(f)-V_{2^{m}}(f)\right\|_{p_{0}},
\end{aligned}
$$

where $p_{0}>1$. We set $\Lambda_{2^{n}}:=2^{-n \varepsilon / 2} \max _{1 \leqslant k \leqslant n} \frac{\left|\lambda_{2^{k}}\right|}{2^{k(\rho-\varepsilon / 2)}}$. Using Nikol'skii's inequality, we get

$$
\begin{aligned}
\left|\lambda_{2^{m}}\right|\left\|V_{2^{m+1}}(f)-V_{2^{m}}(f)\right\|_{p_{0}} & \leqslant 2^{m \rho} \Lambda_{2^{m}}\left\|V_{2^{m+1}}(f)-V_{2^{m}}(f)\right\|_{p_{0}} \\
& \lesssim 2^{m \rho} \Lambda_{2^{m}} 2^{m\left(\theta-\theta_{0}\right)}\left\|V_{2^{m+1}}(f)-V_{2^{m}}(f)\right\|_{1} .
\end{aligned}
$$


Finally, we estimate $I_{1}$ as follows:

$$
I_{1} \lesssim \sum_{m=n}^{\infty} 2^{m(\rho+\theta)} \Lambda_{2^{m}} \omega_{\alpha+\rho+\theta-\varepsilon}\left(f, \frac{1}{2^{m}}\right)_{1} .
$$

Arguing as above, we estimate $I_{2}$. Applying Nikol'skii's inequality with $1<p_{0}<q_{0}<\infty, \theta_{0}=\frac{1}{p_{0}}-\frac{1}{q_{0}}=\theta-\frac{\varepsilon}{2}$ and then Hardy-Littlewood fractional integration theorem and Lemma 1.5.3, we obtain

$$
\begin{aligned}
I_{2}=2^{-n \alpha}\left\|V_{2^{n}}^{(\alpha)}\left(f^{(\lambda, \beta)}\right)\right\|_{\infty} & \lesssim 2^{-n \alpha} 2^{n \frac{1}{q_{0}}}\left\|V_{2^{n}}^{(\alpha)}\left(f^{(\lambda, \beta)}\right)\right\|_{q_{0}} \\
& \lesssim 2^{-n \alpha} 2^{n \frac{1}{q_{0}}}\left\|V_{2^{n}}^{\left(\alpha+\theta_{0}\right)}\left(f^{(\lambda, \beta)}\right)\right\|_{p_{0}} \\
& \lesssim 2^{n\left(\frac{1}{q_{0}}-\alpha\right)} \max _{1 \leqslant k \leqslant n} \frac{\left|\lambda_{2^{k}}\right|}{2^{k(\rho-\varepsilon / 2)}}\left\|V_{2^{n}}^{\left(\alpha+\theta_{0}+\rho-\varepsilon / 2\right)}(f)\right\|_{p_{0} .} .
\end{aligned}
$$

Using again Nikol'skii's inequality gives

$$
\begin{aligned}
& I_{2} \lesssim 2^{n\left(\varepsilon / 2-\alpha+\frac{1}{q_{0}}\right)} \Lambda_{2^{n}} 2^{\left(1-\frac{1}{p_{0}}\right) n}\left\|V_{2^{n}}^{\left(\alpha+\theta_{0}+\rho-\varepsilon / 2\right)}(f)\right\|_{1} \\
& \lesssim 2^{n\left(\varepsilon / 2-\alpha+\theta-\theta_{0}\right)} \Lambda_{2^{n}} 2^{n\left(\alpha+\theta_{0}+\rho-\varepsilon / 2\right)} \omega_{\alpha+\rho+\theta-\varepsilon / 2-\varepsilon / 2}\left(f, \frac{1}{2^{n}}\right)_{1} \\
& =2^{n(\theta+\rho)} \Lambda_{2^{n}} \omega_{\alpha+\rho+\theta-\varepsilon}\left(f, \frac{1}{2^{n}}\right)_{1} .
\end{aligned}
$$

The last inequality follows from Lemma 1.5.2. Collecting estimates for $I_{1}$ and $I_{2}$, we have

$$
\omega_{\alpha}\left(f^{(\lambda, \beta)}, \frac{1}{2^{n}}\right)_{\infty} \lesssim \sum_{m=n}^{\infty} 2^{m(\theta+\rho)} \Lambda_{2^{m}} \omega_{\alpha+\rho+\theta-\varepsilon}\left(f, \frac{1}{2^{m}}\right)_{1} .
$$

Now we state the sharp Ul'yanov inequality in the case $p=1, q=\infty$, and $\theta=1$.

Theorem 2.7.2. Let $\alpha \in \mathbb{R}_{+}$and $\rho \in \mathbb{R}_{+} \cup\{0\}$. Let $f \in L_{1}, \lambda=\left\{\lambda_{n}\right\}_{n=1}^{\infty} \in$ $G M,\left\{\Delta \lambda_{n}\right\} \in G M$, and $\left\{\Delta \frac{\lambda_{n}}{n^{\rho}}\right\} \in G M$. We have

$$
\begin{aligned}
\omega_{\alpha}\left(f^{(\lambda, \beta)}, \frac{1}{2^{n}}\right)_{\infty} & \lesssim \sum_{m=n}^{\infty} 2^{m}\left|\lambda_{2^{m}}\right| \omega_{\alpha+\rho+1}\left(f, \frac{1}{2^{m}}\right)_{1} \\
& +2^{-n \alpha} \sum_{k=1}^{2^{n+2}-1}\left|\frac{\lambda_{k}}{k^{\rho}}-\frac{\lambda_{k+1}}{(k+1)^{\rho}}\right| k^{\rho+\alpha+1} \omega_{\alpha+\rho+1}\left(f, \frac{1}{k}\right)_{1} \\
& +2^{-n \alpha}\left|\sin \frac{\pi(\beta-\rho)}{2}\right| \sum_{k=1}^{n}\left|\lambda_{2^{k}}\right| 2^{k(\alpha+1)} \omega_{\alpha+\rho+1}\left(f, \frac{1}{2^{k}}\right)_{1}
\end{aligned}
$$


Proof of Theorem 2.7.2. Let $1=p$ and $q=\infty$. Using Lemma 1.2.3, we get $\omega_{\alpha}\left(f^{(\lambda, \beta)}, \frac{1}{2^{n}}\right)_{\infty} \lesssim\left\|f^{(\lambda, \beta)}-V_{2^{n}}\left(f^{(\lambda, \beta)}\right)\right\|_{\infty}+2^{-n \alpha}\left\|V_{2^{n}}^{(\alpha)}\left(f^{(\lambda, \beta)}\right)\right\|_{\infty}=: I_{1}+I_{2}$.

Taking into account Lemma 2.3.3 for $q=\infty, \quad q_{1}=1, \quad p_{0}>1$ and $\sigma=$ $2^{n}, L=l-n$, we obtain

$$
\left\|V_{2^{l}}\left(f^{(\lambda, \beta)}\right)-V_{2^{n}}\left(f^{(\lambda, \beta)}\right)\right\|_{\infty} \lesssim \sum_{m=n}^{l-1} 2^{m \frac{1}{p_{0}}}\left\|V_{2^{m+1}}\left(f^{(\lambda, \beta)}\right)-V_{2^{m}}\left(f^{(\lambda, \beta)}\right)\right\|_{p_{0}} .
$$

By the Marcinkiewicz multiplier theorem and properties of $G M$ sequences, we have

$$
\left\|V_{2^{m+1}}\left(f^{(\lambda, \beta)}\right)-V_{2^{m}}\left(f^{(\lambda, \beta)}\right)\right\|_{p_{0}} \lesssim\left|\lambda_{2^{m}}\right|\left\|V_{2^{m+1}}(f)-V_{2^{m}}(f)\right\|_{p_{0}} .
$$

Nikol'skii inequality implies

$$
\left|\lambda_{2^{m}}\right|\left\|V_{2^{m}}(f)-V_{2^{m}}(f)\right\|_{p_{0}} \lesssim\left|\lambda_{2^{m}}\right| 2^{m\left(1-\frac{1}{p_{0}}\right)}\left\|V_{2^{m+1}}(f)-V_{2^{m}}(f)\right\|_{1} .
$$

Applying Jackson's inequality, we obtain

$$
I_{1} \lesssim \sum_{m=n}^{\infty} 2^{m}\left|\lambda_{2^{m}}\right| E_{2^{m}}(f)_{1} \lesssim \sum_{m=n}^{\infty} 2^{m}\left|\lambda_{2^{m}}\right| \omega_{\alpha+\rho+1}\left(f, \frac{1}{2^{m}}\right)_{1}
$$

To estimate $I_{2}$, we first use Lemma 2.3.2

$$
\left\|V_{2^{n}}^{(\alpha)}\left(f^{(\lambda, \beta)}\right)\right\|_{\infty} \lesssim\left\|V_{2^{n}}^{(\alpha+1)}\left(f^{(\lambda, \beta)}\right)\right\|_{1} .
$$

Further using (1.7.48), we have

$$
\begin{aligned}
\left\|V_{2^{n}}^{(\alpha+1)}\left(f^{(\lambda, \beta)}\right)\right\|_{1} & \lesssim \sum_{\mu=1}^{2^{n+2}-1}\left|\frac{\lambda_{\mu}}{\mu^{\rho}}-\frac{\lambda_{\mu+1}}{(\mu+1)^{\rho}}\right| \mu^{\alpha+\rho+1} \omega_{\alpha+\rho+1}\left(f, \frac{1}{\mu}\right)_{1} \\
& +\left|\lambda_{2^{n+2}}\right| 2^{n(\alpha+1)} \omega_{\alpha+\rho+1}\left(f, \frac{1}{2^{n}}\right)_{1} \\
& +\left|\sin \frac{\pi(\beta-\rho)}{2}\right| \sum_{k=0}^{n-1}\left|\lambda_{2^{k+2}}\right| 2^{k(\alpha+1)} \omega_{\alpha+\rho+1}\left(f, \frac{1}{2^{k}}\right)_{1}
\end{aligned}
$$

By properties of $G M$ sequences and collecting estimates of $I_{1}$ and $I_{2}$, we obtain 


$$
\begin{aligned}
\omega_{\alpha}\left(f^{(\lambda, \beta)}, \frac{1}{2^{n}}\right)_{\infty} & \lesssim \sum_{m=n}^{\infty} 2^{m}\left|\lambda_{2^{m}}\right| \omega_{\alpha+\rho+1}\left(f, \frac{1}{2^{m}}\right)_{1} \\
& +2^{-n \alpha} \sum_{k=1}^{2^{n+2}-1}\left|\frac{\lambda_{k}}{k^{\rho}}-\frac{\lambda_{k+1}}{(k+1)^{\rho}}\right| k^{\rho+\alpha+1} \omega_{\alpha+\rho+1}\left(f, \frac{1}{k}\right)_{1} \\
& +2^{-n \alpha}\left|\sin \frac{\pi(\beta-\rho)}{2}\right| \sum_{k=1}^{n}\left|\lambda_{2^{k}}\right| 2^{k(\alpha+1)} \omega_{\alpha+\rho+1}\left(f, \frac{1}{2^{k}}\right)_{1}
\end{aligned}
$$

The proof of Theorem 2.7.2 is now complete.

Let us apply Theorems 2.7.1 and 2.7.2 for power-logarithmic derivatives.

Example 2.7.1. Let $f \in L_{1}, \theta=1$, and

$$
\lambda_{n}=n^{\rho} \ln ^{\gamma}(n+1), \quad \rho>0, \gamma \in \mathbb{R} .
$$

Theorem 2.7.1 implies that

$$
\omega_{\alpha}\left(f^{(\lambda, \beta)}, \frac{1}{2^{n}}\right)_{\infty} \lesssim \sum_{m=n}^{\infty} 2^{m(\theta+\rho)} m^{\gamma} \omega_{\alpha+\rho+\theta-\varepsilon}\left(f, \frac{1}{2^{m}}\right)_{1}
$$

On the other hand, Theorem 2.7.2 implies

$$
\begin{aligned}
& \text { (i) if } \gamma=0, \beta=\rho \\
& \omega_{\alpha}\left(f^{(\lambda, \beta)}, \frac{1}{2^{n}}\right)_{\infty} \lesssim \sum_{m=n}^{\infty} 2^{m(\theta+\rho)} \omega_{\alpha+\rho+\theta}\left(f, \frac{1}{2^{m}}\right)_{1}, \\
& \text { (ii) if } \gamma=0, \\
& \omega_{\alpha}\left(f^{(\lambda, \beta)}, \frac{1}{2^{n}}\right)_{\infty} \lesssim \sum_{m=n}^{\infty} 2^{m(\theta+\rho)} \omega_{\alpha+\rho+\theta}\left(f, \frac{1}{2^{m}}\right)_{1} \\
& +2^{-n \alpha}\left|\sin \frac{\pi(\beta-\rho)}{2}\right| \sum_{k=1}^{n} 2^{k(\alpha+\theta+\rho)} \omega_{\alpha+\rho+\theta}\left(f, \frac{1}{2^{k}}\right)_{1},
\end{aligned}
$$




$$
\begin{aligned}
\text { (iii) if } \gamma \neq 0, & \\
\omega_{\alpha}\left(f^{(\lambda, \beta)}, \frac{1}{2^{n}}\right)_{\infty} & \lesssim \sum_{m=n}^{\infty} 2^{m(\theta+\rho)} m^{\gamma} \omega_{\alpha+\rho+\theta}\left(f, \frac{1}{2^{m}}\right)_{1} \\
& +2^{-n \alpha} \sum_{k=1}^{n} 2^{k(\rho+\theta+\alpha)} k^{\gamma-1} \omega_{\alpha+\rho+\theta}\left(f, \frac{1}{2^{k}}\right)_{1} \\
& +2^{-n \alpha}\left|\sin \frac{\pi(\beta-\rho)}{2}\right| \sum_{k=1}^{n} 2^{k(\alpha+\theta+\rho)} k^{\gamma} \omega_{\alpha+\rho+\theta}\left(f, \frac{1}{2^{k}}\right)_{1} .
\end{aligned}
$$

Proof. The proofs of (2.7.10) and (2.7.11) are clear. The part (iii) follows from Theorem 2.7.2 and the fact that

$\sum_{k=2^{n}}^{2^{n+1}-1}\left|\ln ^{\gamma} k-\ln ^{\gamma}(k+1)\right| k^{\rho+\alpha+1} \omega_{\alpha+\rho+1}\left(f, \frac{1}{k}\right)_{1} \asymp n^{\gamma-1} 2^{n(\rho+\alpha+1)} \omega_{\alpha+\rho+1}\left(f, \frac{1}{2^{n}}\right)_{1}$.

Remark 2.7.1. Note that inequality (2.7.10) and (2.7.11) imply the following estimates:

$$
\omega_{\alpha}\left(f^{(\rho)}, \delta\right)_{\infty} \lesssim \int_{0}^{\delta} t^{-\theta-\rho} \omega_{\alpha+\rho+1}(f, t)_{1} \frac{d t}{t}
$$

and

$$
\omega_{\alpha}\left(\tilde{f}^{(\rho)}, \delta\right)_{\infty} \lesssim \int_{0}^{\delta} t^{-\theta-\rho} \omega_{\alpha+\rho+1}(f, t)_{1} \frac{d t}{t}+t^{\alpha} \int_{\delta}^{1} t^{-\theta-\rho-\alpha} \omega_{\alpha+\rho+1}(f, t)_{1} \frac{d t}{t} .
$$

Here the first inequality is given in Theorem B4 and the second one is new.

\subsection{Estimates for the best approximations}

In this subsection we obtain estimates for the $L_{q}$-best approximations of the generalized Liouville-Weyl derivatives via the $L_{p}$-best approximations of the function themselves.

Theorem 2.8.1. Let $f \in L_{p}, 1<p<q \leqslant \infty, \theta=1 / p-1 / q$. Let $\lambda=$ $\left\{\lambda_{n}\right\}_{n=1}^{\infty} \in G M$. Then, for any $\alpha>0$,

$$
E_{2^{n}}\left(f^{(\lambda, \beta)}\right)_{q} \lesssim\left(\sum_{m=n}^{\infty}\left(2^{m \theta}\left|\lambda_{2^{m}}\right| E_{2^{m}}(f)_{p}\right)^{q_{1}}\right)^{1 / q_{1}}
$$


where $q_{1}= \begin{cases}q, & q<\infty \\ 1, & q=\infty\end{cases}$

Proof of Theorem 2.8.1. Let $1<p<q \leqslant \infty$. By Lemmas 2.3.3 and 1.5.3, we have

$$
\begin{aligned}
& \left\|S_{2^{l}}\left(f^{(\lambda, \beta)}\right)-S_{2^{n}}\left(f^{(\lambda, \beta)}\right)\right\|_{q} \lesssim\left(\sum_{m=n}^{l-1}\left(2^{m \theta}\left\|S_{2^{m+1}}\left(f^{(\lambda, \beta)}\right)-S_{2^{m}}\left(f^{(\lambda, \beta)}\right)\right\|_{p}\right)^{q_{1}}\right)^{1 / q_{1}} \\
& \lesssim\left(\sum_{m=n}^{l-1}\left(2^{m \theta}\left|\lambda_{2^{m}}\right|\left\|S_{2^{m+1}}(f)-S_{2^{m}}(f)\right\|_{p}\right)^{q_{1}}\right)^{1 / q_{1}} \\
& \lesssim\left(\sum_{m=n}^{\infty}\left(2^{m \theta}\left|\lambda_{2^{m}}\right| E_{2^{m}}(f)_{p}\right)^{q_{1}}\right)^{1 / q_{1}}
\end{aligned}
$$

where $q_{1}=\left\{\begin{array}{ll}q, & q<\infty, \\ 1, & q=\infty .\end{array}\right.$ Using the inequality $E_{n}\left(f^{(\lambda, \beta)}\right)_{q} \lesssim \| f^{(\lambda, \beta)}-$ $S_{n}\left(f^{(\lambda, \beta)}\right) \|_{q}$ and the fact that $\left\|f^{(\lambda, \beta)}-S_{2^{n}}\left(f^{(\lambda, \beta)}\right)\right\|_{q} \leqslant \lim _{l \rightarrow \infty} \| S_{2^{l}}\left(f^{(\lambda, \beta)}\right)-$ $S_{2^{n}}\left(f^{(\lambda, \beta)}\right) \|_{q}$, we obtain

$$
E_{2^{n}}\left(f^{(\lambda, \beta)}\right)_{q} \lesssim\left(\sum_{m=n}^{\infty}\left(2^{m \theta}\left|\lambda_{2^{m}}\right| E_{2^{m}}(f)_{p}\right)^{q_{1}}\right)^{1 / q_{1}} .
$$

Theorem 2.8.2. Let $f \in L_{p}, 1=p<q \leqslant \infty, \theta=1-1 / q$. Let $\lambda=$ $\left\{\lambda_{n}\right\}_{n=1}^{\infty} \in G M$. Then, for any $\alpha>0$,

$$
E_{2^{n+1}-1}\left(f^{(\lambda, \beta)}\right)_{q} \lesssim\left(\sum_{m=n}^{\infty}\left(2^{m \theta}\left|\lambda_{2^{m}}\right| E_{2^{m}}(f)_{p}\right)^{q_{1}}\right)^{1 / q_{1}},
$$

where $q_{1}= \begin{cases}q, & q<\infty \\ 1, & q=\infty\end{cases}$

Proof of Theorem 2.8.2. The proof follows from estimate $I_{1}$ in Theorem 2.7.2 when $1<q \leqslant \infty$. $\left\|f^{(\lambda, \beta)}-V_{2^{n}}\left(f^{(\lambda, \beta)}\right)\right\|_{q} \leqslant \lim _{l \rightarrow \infty}\left\|V_{2^{l}}\left(f^{(\lambda, \beta)}\right)-V_{2^{n}}\left(f^{(\lambda, \beta)}\right)\right\|_{q}$, applying the inequality $E_{2^{n+1}-1}\left(f^{(\lambda, \beta)}\right)_{q} \lesssim\left\|f^{(\lambda, \beta)}-V_{2^{n}}\left(f^{(\lambda, \beta)}\right)\right\|_{q}$, we get

$$
E_{2^{n+1}-1}\left(f^{(\lambda, \beta)}\right)_{q} \lesssim\left(\sum_{m=n}^{\infty}\left(2^{m \theta}\left|\lambda_{2^{m}}\right| E_{2^{m}}(f)_{1}\right)^{q_{1}}\right)^{1 / q_{1}} .
$$


Remark 2.8.1. Note that some estimates of the best approximation of $f^{(\lambda, \beta)}$ in $L_{p}$ in terms of the best approximation of $f$ in $L_{p}$ were obtained in the paper [54]. 


\section{Liouville-Weyl derivatives of double trigonometric series}

\subsection{Some notations, known results, and goals}

Let $L_{p}\left(\mathbb{T}^{2}\right), 1<p<\infty$, be the space of measureable functions of two variables that are $2 \pi$-periodic in each variable and such that

$$
\|f\|_{p}=\left(\int_{0}^{2 \pi} \int_{0}^{2 \pi}\left|f\left(x_{1}, x_{2}\right)\right|^{p} d x_{1} d x_{2}\right)^{\frac{1}{p}}<\infty .
$$

$L_{p}^{0}$ - the set of functions $f \in L_{p}$ such that

$$
\int_{0}^{2 \pi} f\left(x_{1}, x_{2}\right) d x_{2}=0 \text { a.e. } x_{1}
$$

and

$$
\int_{0}^{2 \pi} f\left(x_{1}, x_{2}\right) d x_{1}=0 \text { a.e. } x_{2} .
$$

Let $f^{\left(\rho_{1}, \rho_{2}\right)}$ be a derivative in the sense of Weyl of the function $f\left(x_{1}, x_{2}\right)$ of order $\rho_{1}\left(\rho_{1} \geqslant 0\right)$ with respect to $x_{1}$ and of order $\rho_{2}\left(\rho_{2} \geqslant 0\right)$ with respect to $x_{2}$.

In the paper [48], the following result was proved.

Theorem C. Let $1<p<\infty, 0<\theta \leqslant \min (2, p), \max (2, p) \leqslant \tau<\infty$, and $r_{1}, r_{2}, \beta_{1}, \beta_{2}$ be positive numbers.

A. Let $f \in L_{p}^{0}\left(\mathbb{T}^{2}\right)$ and

$$
J_{1}(f, \theta):=\left(\int_{0}^{1} \int_{0}^{1} t_{1}^{-r_{1} \theta-1} t_{2}^{-r_{2} \theta-1} \omega_{r_{1}+\beta_{1}, r_{2}+\beta_{2}}^{\theta}\left(f, t_{1}, t_{2}\right)_{p} d t_{1} d t_{2}\right)^{\frac{1}{\theta}}<\infty,
$$

then the mixed derivative of $f$ in the sense of Weil $f^{\left(r_{1}, r_{2}\right)} \in L_{p}^{0}\left(\mathbb{T}^{2}\right)$, and

$$
\left\|f^{\left(r_{1}, r_{2}\right)}\right\|_{p} \leqslant C J_{1}(f, \theta) .
$$

B. Let $f \in L_{p}^{0}\left(\mathbb{T}^{2}\right)$ be such that $f^{\left(r_{1}, r_{2}\right)} \in L_{p}^{0}\left(\mathbb{T}^{2}\right)$, then

$$
J_{1}(f, \tau) \leqslant C\left\|f^{\left(r_{1}, r_{2}\right)}\right\|_{p} .
$$


Here $\omega_{l_{1}, l_{2}}\left(f, t_{1}, t_{2}\right)_{p}$ is the mixed modulus of smoothness of a function $f \in L_{p}\left(\mathbb{T}^{2}\right)$ of orders $l_{1}$ and $l_{2}$ with respect to variables $x_{1}$ and $x_{2}$, correspondingly, that is,

$$
\omega_{l_{1}, l_{2}}\left(f, \delta_{1}, \delta_{2}\right)_{p}=\sup _{\left|h_{1}\right| \leqslant \delta_{1},\left|h_{2}\right| \leqslant \delta_{2}}\left\|\Delta_{h_{1}}^{l_{1}}\left(\Delta_{h_{2}}^{l_{2}}(f)\right)\right\|_{p} .
$$

The difference of order $l_{1}>0$ with respect to the variable $x_{1}$ and the difference of order $l_{2}>0$ with respect to the variable $x_{2}$ are defined as follows:

$$
\Delta_{h_{1}}^{l_{1}}(f)=\sum_{k_{1}=0}^{\infty}(-1)^{k_{1}} C_{l_{1}}^{k_{1}} f\left(x_{1}+\left(l_{1}-k_{1}\right) h_{1}, x_{2}\right)
$$

and

$$
\Delta_{h_{2}}^{l_{2}}(f)=\sum_{k_{2}=0}^{\infty}(-1)^{k_{2}} C_{l_{2}}^{k_{2}} f\left(x_{1}, x_{2}+\left(l_{2}-k_{2}\right) h_{2}\right) .
$$

Let $Y_{m_{1}, m_{2}}(f)_{p}$ be the best approximation by a two-dimensional angle of the function $f \in L_{p}\left(\mathbb{T}^{2}\right)$, i.e.,

$$
Y_{m_{1}, m_{2}}(f)_{p}=\inf _{T_{m_{1}, \infty}, T_{\infty, m_{2}}}\left\|f-T_{m_{1}, \infty}-T_{\infty, m_{2}}\right\|_{p}
$$

where the function $T_{m_{1}, \infty}\left(x_{1}, x_{2}\right) \in L_{p}\left(\mathbb{T}^{2}\right)$ is a trigonometric polynomial of degree at most $m_{1}$ in $x_{1}$, and the function $T_{\infty, m_{2}}\left(x_{1}, x_{2}\right) \in L_{p}\left(\mathbb{T}^{2}\right)$ is a trigonometric polynomial of degree at most $m_{2}$ in $x_{2}$.

The direct and inverse theorems between best approximations by twodimensional angle and mixed moduli of smoothness are well known $[44,45]$. The Jackson inequality reads as follows: if $f \in L_{p}^{0}\left(\mathbb{T}^{2}\right), 1<p<\infty$, then

$$
Y_{m_{1}, m_{2}}(f)_{p} \lesssim \omega_{\alpha_{1}, \alpha_{2}}\left(f ; \frac{1}{m_{1}}, \frac{1}{m_{2}}\right)_{p} .
$$

The inverse inequality states that if $f \in L_{p}^{0}\left(\mathbb{T}^{2}\right), 1<p<\infty$, then

$$
\omega_{\alpha_{1}, \alpha_{2}}\left(f ; \frac{1}{m_{1}}, \frac{1}{m_{2}}\right)_{p} \lesssim \frac{1}{m_{1}^{\alpha_{1}} m_{2}^{\alpha_{2}}} \sum_{k_{1}=0}^{m_{1}} \sum_{k_{2}=0}^{m_{2}}\left(k_{1}+1\right)^{\alpha_{1}-1}\left(k_{2}+1\right)^{\alpha_{2}-1} Y_{k_{1}, k_{2}}(f)_{p} .
$$

Using these estimates and applying Hardy's inequalities, we easily obtain that

$$
J_{1}(f, s) \asymp\left(\sum_{k_{1}=0}^{\infty} \sum_{k_{2}=0}^{\infty}\left(k_{1}+1\right)^{r_{1} s-1}\left(k_{2}+1\right)^{r_{2} s-1} Y_{k_{1}, k_{2}}^{s}(f)_{p}\right)^{\frac{1}{s}}
$$


for any $s>0$, where as usual $F_{1}(f, r, s, p) \asymp F_{2}(f, r, s, p)$ means that there exist positive constants $C_{1}$ and $C_{2}$, independent of $f$ such that

$$
C_{1} \cdot F_{1}(f, r, s, p) \leqslant F_{2}(f, r, s, p) \leqslant C_{2} \cdot F_{1}(f, r, s, p) .
$$

The goal of this section is to extend theorem $\mathrm{C}$ in the following respects. First, we consider the generalized Liouville-Weyl derivatives in place of the classical mixed Weyl derivatives. Second, similarly to one-dimensional inequalities (1.6.29) and (1.6.30), we obtain estimates of the angle approximations of these derivatives by angle approximation of functions themselves. By $\sigma(f)$ we will denote the Fourier series of a function $f \in L_{p}\left(\mathbb{T}^{2}\right)$, that is,

$$
\begin{array}{r}
\sigma(f) \equiv \sum_{n_{1}=0}^{\infty} \sum_{n_{2}=0}^{\infty}\left(a_{n_{1} n_{2}} \cos n_{1} x_{1} \cos n_{2} x_{2}+b_{n_{1} n_{2}} \cos n_{1} x_{1} \sin n_{2} x_{2}+\right. \\
\left.+c_{n_{1} n_{2}} \sin n_{1} x_{1} \cos n_{2} x_{2}+d_{n_{1} n_{2}} \sin n_{1} x_{1} \sin n_{2} x_{2}\right)
\end{array}
$$

where for the sake of brevity we assume that $\cos (0 \cdot t)=\frac{1}{2}$.

The transformed Fourier series of $\sigma(f)$ is given by

$$
\begin{aligned}
\sigma\left(f, \lambda, \beta_{1}, \beta_{2}\right) \equiv \sum_{n_{1}=1}^{\infty} \sum_{n_{2}=1}^{\infty} \lambda_{n_{1}, n_{2}} & a_{n_{1} n_{2}} \cos \left(n_{1} x_{1}+\beta_{1} \pi / 2\right) \cos \left(n_{2} x_{2}+\beta_{2} \pi / 2\right) \\
& +b_{n_{1} n_{2}} \cos \left(n_{1} x_{1}+\beta_{1} \pi / 2\right) \sin \left(n_{2} x_{2}+\beta_{2} \pi / 2\right) \\
& +c_{n_{1} n_{2}} \sin \left(n_{1} x_{1}+\beta_{1} \pi / 2\right) \cos \left(n_{2} x_{2}+\beta_{2} \pi / 2\right) \\
& \left.+d_{n_{1} n_{2}} \sin \left(n_{1} x_{1}+\beta_{1} \pi / 2\right) \sin \left(n_{2} x_{2}+\beta_{2} \pi / 2\right)\right),
\end{aligned}
$$

where $\beta_{1}, \beta_{2} \in \mathbb{R}$ and $\lambda=\left\{\lambda_{n_{1} n_{2}}\right\}_{n_{1}, n_{2} \in \mathbb{N}}$ is a sequence of real numbers.

By analogy with the one-dimensional case we call the function $\varphi\left(x_{1}, x_{2}\right) \sim \sigma\left(f, \lambda, \beta_{1}, \beta_{2}\right)$ the $\left(\lambda, \beta_{1}, \beta_{2}\right)-$ mixed derivative of the function $f$ (or Liouville-Weyl derivative) and denote it by $f^{\left(\lambda, \beta_{1}, \beta_{2}\right)}\left(x_{1}, x_{2}\right)$. For example, if $\lambda_{n_{1} n_{2}}=n_{1}^{r_{1}} n_{2}^{r_{2}}, r_{i} \geqslant 0, \beta_{i}=r_{i}(i=1,2)$, then $f^{\left(\lambda, \beta_{1}, \beta_{2}\right)}=f^{\left(r_{1}, r_{2}\right)}$, where $f^{\left(r_{1}, r_{2}\right)}$ is the mixed derivative of $f$ in the sense of Weyl. Note that, for any $\beta_{1}$ and $\beta_{2},\left\|f^{\left(\lambda, \beta_{1}, \beta_{2}\right)}\right\|_{p} \asymp\left\|f^{(\lambda, 0,0)}\right\|_{p}, 1<p<\infty$.

We recall [69] that a sequence $\lambda:=\left\{\lambda_{n}\right\}_{n=1}^{\infty}$ is said to be general monotone, written $\lambda \in G M$, if the relation

$$
\sum_{k=n}^{2 n}\left|\lambda_{k}-\lambda_{k+1}\right| \leqslant C\left|\lambda_{n}\right|
$$


holds for all integer $n$, where the constant $C$ is independent of $n$.

Similarly, one can introduce the class $G M^{2}$, where 2 stands for dimension. See $[14,15,16]$.

Definition 3.1.1. A sequence $\lambda=\left\{\lambda_{n_{1} n_{2}}\right\}_{n_{1}, n_{2} \in N}$ is said to be general monotone, written $\lambda \in G M^{2}$, if the relations

$$
\begin{aligned}
& \sum_{k_{1}=n_{1}}^{2 n_{1}}\left|\lambda_{k_{1}, n_{2}}-\lambda_{k_{1}+1, n_{2}}\right| \leqslant C\left|\lambda_{n_{1}, n_{2}}\right|, \\
& \sum_{k_{2}=n_{2}}^{2 n_{2}}\left|\lambda_{n_{1}, k_{2}}-\lambda_{n_{1}, k_{2}+1}\right| \leqslant C\left|\lambda_{n_{1}, n_{2}}\right|, \\
& \sum_{k_{1}=n_{1}}^{2 n_{1}} \sum_{k_{2}=n_{2}}^{2 n_{2}}\left|\lambda_{k_{1}, k_{2}}-\lambda_{k_{1}+1, k_{2}}-\lambda_{k_{1}, k_{2}+1}+\lambda_{k_{1}+1, k_{2}+1}\right| \leqslant C\left|\lambda_{n_{1}, n_{2}}\right|
\end{aligned}
$$

hold for all integers $n_{1}$ and $n_{2}$, where the constant $C$ is independent of $n_{1}$ and $n_{2}$.

\subsection{Auxiliary results}

In this section we state several useful lemmas that will be used in the proof our main result. First, we introduce some notation.

Let the series

$$
\begin{aligned}
\sigma(f) & :=\sum_{n_{1}=1}^{\infty} \sum_{n_{2}=1}^{\infty}\left(a_{n_{1} n_{2}} \cos n_{1} x_{1} \cos n_{2} x_{2}+b_{n_{1} n_{2}} \cos n_{1} x_{1} \sin n_{2} x_{2}\right. \\
& \left.+c_{n_{1} n_{2}} \sin n_{1} x_{1} \cos n_{2} x_{2}+d_{n_{1} n_{2}} \sin n_{1} x_{1} \sin n_{2} x_{2}\right)=\sum_{n_{1}=1}^{\infty} \sum_{n_{2}=1}^{\infty} A_{n_{1} n_{2}}\left(x_{1}, x_{2}\right) .
\end{aligned}
$$

be the Fourier series of $f \in L_{p}^{0}\left(\mathbb{T}^{2}\right)$.

We denote

$$
\Delta_{m_{1} m_{2}}:=\sum_{n_{1}=2^{m_{1}-1}}^{2^{m_{1}}-1} \sum_{n_{2}=2^{m_{2}-1}}^{2^{m_{2}-1}} A_{n_{1} n_{2}}\left(x_{1}, x_{2}\right), \quad m_{1}, m_{2}=1,2, \ldots
$$


Let $S_{m_{1}, \infty}(f), S_{\infty, m_{2}}(f), S_{m_{1}, m_{2}}(f)$ denote the partial sums of Fourier series of $f\left(x_{1}, x_{2}\right)$, i.e.,

$$
\begin{aligned}
& S_{m_{1}, \infty}(f)=\frac{1}{\pi} \int_{0}^{2 \pi} f\left(x_{1}+t_{1}, x_{2}\right) D_{m_{1}}\left(t_{1}\right) d t_{1}, \\
& S_{\infty, m_{2}}(f)=\frac{1}{\pi} \int_{0}^{2 \pi} f\left(x_{1}, x_{2}+t_{2}\right) D_{m_{2}}\left(t_{2}\right) d t_{2}, \\
& S_{m_{1}, m_{2}}(f)=\frac{1}{\pi^{2}} \int_{0}^{2 \pi} \int_{0}^{2 \pi} f\left(x_{1}+t_{1}, x_{2}+t_{2}\right) D_{m_{1}}\left(t_{1}\right) D_{m_{2}}\left(t_{2}\right) d t_{1} d t_{2},
\end{aligned}
$$

where $D_{m}(t)=\frac{\sin \left(m+\frac{1}{2}\right) t}{2 \sin \frac{t}{2}}, m \in \mathbb{N} \cup\{0\}$.

From [69], it follows that if $\left\{\lambda_{n_{1} n_{2}}\right\} \in G M^{2}$, then

$$
\left|\lambda_{k_{1}, k_{2}}\right| \leqslant C\left|\lambda_{n_{1}, n_{2}}\right| \text { for } n_{1} \leqslant k_{1} \leqslant 2 n_{1}, \quad n_{2} \leqslant k_{2} \leqslant 2 n_{2} .
$$

This implies that the condition

$$
\sum_{k_{1}=n_{1}}^{2 n_{1}} \sum_{k_{2}=n_{2}}^{2 n_{2}}\left|\lambda_{k_{1}, k_{2}}-\lambda_{k_{1}+1, k_{2}}-\lambda_{k_{1}, k_{2}+1}+\lambda_{k_{1}+1, k_{2}+1}\right| \leqslant C\left(\left|\lambda_{n_{1}, n_{2}}\right|+\left|\lambda_{2 n_{1}, 2 n_{2}}\right|\right)
$$

is equivalent to the condition

$$
\sum_{k_{1}=n_{1}}^{2 n_{1}} \sum_{k_{2}=n_{2}}^{2 n_{2}}\left|\lambda_{k_{1}, k_{2}}-\lambda_{k_{1}+1, k_{2}}-\lambda_{k_{1}, k_{2}+1}+\lambda_{k_{1}+1, k_{2}+1}\right| \leqslant C\left|\lambda_{n_{1}, n_{2}}\right| .
$$

Lemma 3.2.1. A sequences $\left\{\lambda_{n_{1} n_{2}}\right\} \in G M^{2}$ satisfies the following properties: there exists $C>0$ such that

(i) $\left|\lambda_{k_{1}, k_{2}}\right| \leqslant C\left|\lambda_{n_{1}, n_{2}}\right|$ for $n_{1} \leqslant k_{1} \leqslant 2 n_{1}, \quad n_{2} \leqslant k_{2} \leqslant 2 n_{2}$,

$$
\begin{aligned}
& \sum_{k_{1}=n_{1}}^{N_{1}}\left|\lambda_{k_{1}, n_{2}}-\lambda_{k_{1}+1, n_{2}}\right| \leqslant C\left(\left|\lambda_{n_{1} n_{2}}\right|+\sum_{k_{1}=n_{1}+1}^{N_{1}} \frac{\left|\lambda_{k_{1} n_{2}}\right|}{k_{1}}\right) \quad \text { for } \quad n_{1}<N_{1}, \\
& \sum_{k_{2}=n_{2}}^{N_{2}}\left|\lambda_{n_{1}, k_{2}}-\lambda_{n_{1}, k_{2}+1}\right| \leqslant C\left(\left|\lambda_{n_{1} n_{2}}\right|+\sum_{k_{2}=n_{2}+1}^{N_{2}} \frac{\left|\lambda_{n_{1} k_{2}}\right|}{k_{2}}\right) \quad \text { for } \quad n_{2}<N_{2},
\end{aligned}
$$


(iv) $\sum_{k_{1}=n_{1}}^{N_{1}} \sum_{k_{2}=n_{2}}^{N_{2}}\left|\lambda_{k_{1}, k_{2}}-\lambda_{k_{1}+1, k_{2}}-\lambda_{k_{1}, k_{2}+1}+\lambda_{k_{1}+1, k_{2}+1}\right| \leqslant$

$\leqslant C\left(\left|\lambda_{n_{1} n_{2}}\right|+\sum_{k_{1}=n_{1}+1}^{N_{1}} \frac{\left|\lambda_{k_{1} n_{2}}\right|}{k_{1}}+\sum_{k_{2}=n_{2}+1}^{N_{2}} \frac{\left|\lambda_{n_{1} k_{2}}\right|}{k_{2}}+\sum_{k_{1}=n_{1}+1}^{N_{1}} \sum_{k_{2}=n_{2}+1}^{N_{2}} \frac{\left|\lambda_{k_{1} k_{2}}\right|}{k_{1} k_{2}}\right)$ for $n_{1}<$ $N_{1}, n_{2}<N_{2}$.

Proof. The properties $(i),(i i)$, and (iii) follow from Lemma 1.4.1. Let us prove the property $(i v)$. Let $l_{1} \in \mathbb{N} \cup\{0\}, l_{2} \in \mathbb{N} \cup\{0\}$ such that $2^{l_{1}} n_{1} \leqslant$ $N_{1}<2^{l_{1}+1} n_{1}, 2^{l_{2}} n_{2} \leqslant N_{2}<2^{l_{2}+1} n_{2}$. Then, by definition, we have

$$
\begin{aligned}
A & =\sum_{k_{1}=n_{1}}^{N_{1}} \sum_{k_{2}=n_{2}}^{N_{2}}\left|\lambda_{k_{1}, k_{2}}-\lambda_{k_{1}+1, k_{2}}-\lambda_{k_{1}, k_{2}+1}+\lambda_{k_{1}+1, k_{2}+1}\right| \\
& \leqslant \sum_{s_{1}=0}^{l_{1}} \sum_{s_{2}=0}^{l_{2}} \sum_{2_{1}=2^{s_{1}} n_{1}}^{2_{1}+1} \sum_{k_{2}=2^{s_{2}} n_{2}}^{2_{s_{2}+1} n_{2}-1}\left|\lambda_{k_{1}, k_{2}}-\lambda_{k_{1}+1, k_{2}}-\lambda_{k_{1}, k_{2}+1}+\lambda_{k_{1}+1, k_{2}+1}\right| \\
& \leqslant C \sum_{s_{1}=0}^{l_{1}} \sum_{s_{2}=0}^{l_{2}}\left|\lambda_{2^{s_{1}} n_{1}, 2^{s_{2}} n_{2}}\right| .
\end{aligned}
$$

Using $(i)$, we get

$$
\begin{aligned}
A & \leqslant C\left(\left|\lambda_{n_{1}, n_{2}}\right|+\sum_{s_{1}=1}^{l_{1}}\left|\lambda_{2^{s_{1}} n_{1}, n_{2}}\right|+\sum_{s_{2}=1}^{l_{2}}\left|\lambda_{n_{1}, 2^{s_{2}} n_{2}}\right|+\sum_{s_{1}=1}^{l_{1}} \sum_{s_{2}=1}^{l_{2}}\left|\lambda_{2^{s_{1}} n_{1}, 2^{s_{2}} n_{2}}\right|\right) \\
& \leqslant C\left(\left|\lambda_{n_{1}, n_{2}}\right|+\sum_{s_{1}=1}^{l_{1}}\left|\lambda_{2^{s_{1}} n_{1}, n_{2}}\right| \sum_{k_{1}=2^{s_{1}-1} n_{1}+1}^{l^{s_{1}} n_{1}} \frac{1}{k_{1}}+\sum_{s_{2}=1}^{l_{2}}\left|\lambda_{n_{1}, 2^{s_{2}} n_{2}}\right| \sum_{k_{2}=2^{s_{2}-1} n_{2}+1}^{2^{s_{2}} n_{2}} \frac{1}{k_{2}}\right. \\
& \left.+\sum_{s_{1}=1}^{l_{1}} \sum_{s_{2}=1}^{l_{2}}\left|\lambda_{2^{s_{1}} n_{1}, 2^{s_{2}} n_{2}}\right| \sum_{k_{1}=2^{s_{1}-1} n_{1}+1}^{\sum_{k_{2}=2^{s_{2}-1} n_{2}+1}} \frac{1}{k_{1}} \frac{1}{k_{2}}\right) \\
& \leqslant C\left(\left|\lambda_{n_{1} n_{2}}\right|+\sum_{k_{1}=n_{1}+1}^{N_{1}} \frac{\left|\lambda_{k_{1} n_{2}}\right|}{k_{1}}+\sum_{k_{2}=n_{2}+1}^{N_{2}} \frac{\left|\lambda_{n_{1} k_{2}}\right|}{k_{2}}+\sum_{k_{1}=n_{1}+1}^{N_{1}} \sum_{k_{2}=n_{2}+1}^{N_{2}} \frac{\left|\lambda_{k_{1} k_{2}}\right|}{k_{1} k_{2}}\right) .
\end{aligned}
$$


Lemma 3.2.2. Let $f \in L_{p}^{0}\left(\mathbb{T}^{2}\right)$ have the Fourier series (3.2.3). We consider

$$
\begin{aligned}
\sigma(\varphi) & \equiv \sum_{n_{1}=1}^{\infty} \sum_{n_{2}=1}^{\infty}\left(a_{n_{1} n_{2}} \cos \left(n_{1} x_{1}+\beta_{1} \pi / 2\right) \cos \left(n_{2} x_{2}+\beta_{2} \pi / 2\right)\right. \\
& +b_{n_{1} n_{2}} \cos \left(n_{1} x_{1}+\beta_{1} \pi / 2\right) \sin \left(n_{2} x_{2}+\beta_{2} \pi / 2\right) \\
& +c_{n_{1} n_{2}} \sin \left(n_{1} x_{1}+\beta_{1} \pi / 2\right) \cos \left(n_{2} x_{2}+\beta_{2} \pi / 2\right) \\
& \left.+d_{n_{1} n_{2}} \sin \left(n_{1} x_{1}+\beta_{1} \pi / 2\right) \sin \left(n_{2} x_{2}+\beta_{2} \pi / 2\right)\right),
\end{aligned}
$$

where $\beta_{1}, \beta_{2} \in \mathbb{R}$. Then

$$
C_{1}(p)\|f\|_{p} \leqslant\|\varphi\|_{p} \leqslant C_{2}(p)\|f\|_{p} .
$$

The proof is analogous to the proof of Lemma 5.2.6 of [49] and it uses boundedness of the conjugate operator in $L_{p}, 1<p<\infty$.

Lemma 3.2.3. [43, $p$. 125] Let $a_{n} \geqslant 0,0<\alpha \leqslant \beta<\infty$. Then

$$
\left(\sum_{\nu=1}^{\infty} a_{\nu}^{\beta}\right)^{\frac{1}{\beta}} \leqslant\left(\sum_{\nu=1}^{\infty} a_{\nu}^{\alpha}\right)^{\frac{1}{\alpha}}
$$

Lemma 3.2.4. $[45,49]$ Let $f \in L_{p}\left(\mathbb{T}^{2}\right), 1<p<\infty$, and $m_{i} \in \mathbb{N} \cup\{0\}(i=$ 1,2). Then

$$
\left\|f-S_{m_{1}, \infty}(f)-S_{\infty, m_{2}}(f)+S_{m_{1}, m_{2}}(f)\right\|_{p} \asymp Y_{m_{1}, m_{2}}(f)_{p} .
$$

Lemma 3.2.5. [43] Then

(a). Let $1<p<\infty$ and (3.2.3) be the Fourier series of $f \in L_{p}^{0}\left(\mathbb{T}^{2}\right)$.

$$
\left(\int_{0}^{2 \pi} \int_{0}^{2 \pi}\left(\sum_{m_{1}=1}^{\infty} \sum_{m_{2}=1}^{\infty} \Delta_{m_{1} m_{2}}^{2}\right)^{\frac{p}{2}} d x_{1} d x_{2}\right)^{\frac{1}{p}} \leqslant C(p)\|f\|_{p} .
$$

(b). Let $1<p<\infty$. If (3.2.3) satisfies the following inequality

$$
I_{p}=\left(\int_{0}^{2 \pi} \int_{0}^{2 \pi}\left(\sum_{m_{1}=1}^{\infty} \sum_{m_{2}=1}^{\infty} \Delta_{m_{1} m_{2}}^{2}\right)^{\frac{p}{2}} d x_{1} d x_{2}\right)^{\frac{1}{p}}<\infty,
$$

then (3.2.3) is the Fourier series of a function $f\left(x_{1}, x_{2}\right) \in L_{p}\left(\mathbb{T}^{2}\right)$ and

$$
\|f\|_{p} \leqslant C(p) I_{p} .
$$


Lemma 3.2.6. [43] Let $f \in L_{p}^{0}\left(\mathbb{T}^{2}\right), 1<p<\infty$, and (3.2.3) be the Fourier series of $f$. If $\left\{\lambda_{n_{1} n_{2}}\right\}$ satisfies the following conditions

(i) $\left|\lambda_{n_{1}, n_{2}}\right| \leqslant M$

(ii) $\sum_{m_{1}=2^{n_{1}-1}}^{2^{n_{1}}-1}\left|\lambda_{m_{1}, n_{2}}-\lambda_{m_{1}+1, n_{2}}\right| \leqslant M, \sum_{m_{2}=2^{n_{2}-1}}^{2^{n_{2}-1}}\left|\lambda_{n_{1}, m_{2}}-\lambda_{n_{1}, m_{2}+1}\right| \leqslant M$,

(iii) $\sum_{m_{1}=2^{n_{1}-1}}^{2^{n_{1}}-1} \sum_{m_{2}=2^{n_{2}-1}}^{2^{n_{2}-1}}\left|\lambda_{m_{1}, m_{2}}-\lambda_{m_{1}+1, m_{2}}-\lambda_{m_{1}, m_{2}+1}+\lambda_{m_{1}+1, m_{2}+1}\right| \leqslant M$ for all $n_{1}, n_{2}=1,2, \ldots$.

Then the series

$$
\sum_{n_{1}=1}^{\infty} \sum_{n_{2}=1}^{\infty} \lambda_{n_{1}, n_{2}} A_{n_{1} n_{2}}\left(x_{1}, x_{2}\right)
$$

is the Fourier series of a function $\varphi(f, \lambda) \in L_{p}^{0}\left(\mathbb{T}^{2}\right)$ and $\|\varphi\|_{p} \leqslant C\|f\|_{p}$, where the constant $C$ is independent of $f$.

3.3 Upper estimates of angle best approximations of generalized Liouville-Weyl derivatives

Our main result reads as follows.

Theorem 3.3.1. Let $1<p<\infty, 0<\theta \leqslant \min (p, 2), \lambda=\left\{\lambda_{n_{1}, n_{2}}\right\}_{n_{1}, n_{2} \in N}$ be a sequence of positive numbers such that $\lambda \in G M^{2}, \alpha_{i} \in \mathbb{R}_{+}, r_{i} \in \mathbb{R}_{+} \cup\{0\}$, and $\beta_{i} \in \mathbb{R}(i=1,2)$.

If for $f \in L_{p}^{0}\left(\mathbb{T}^{2}\right)$ the series

$$
\begin{aligned}
& \sum_{n_{1}=1}^{\infty}\left|\lambda_{n_{1}+1,1}^{\theta}-\lambda_{n_{1}, 1}^{\theta}\right| Y_{n_{1}, 0}^{\theta}(f)_{p}+\sum_{n_{2}=1}^{\infty}\left|\lambda_{1, n_{2}+1}^{\theta}-\lambda_{1, n_{2}}^{\theta}\right| Y_{0, n_{2}}^{\theta}(f)_{p} \\
& +\sum_{k_{1}=1}^{\infty} \sum_{k_{2}=1}^{\infty}\left|\lambda_{k_{1}+1, k_{2}+1}^{\theta}-\lambda_{k_{1}+1, k_{2}}^{\theta}-\lambda_{k_{1}, k_{2}+1}^{\theta}+\lambda_{k_{1}, k_{2}}^{\theta}\right| Y_{k_{1}, k_{2}}^{\theta}(f)_{p}
\end{aligned}
$$

converges, then there exists a function $f^{\left(\lambda, \beta_{1}, \beta_{2}\right)} \in L_{p}^{0}\left(\mathbb{T}^{2}\right)$, with the Fourier series $\sigma\left(f, \lambda, \beta_{1}, \beta_{2}\right)$, and 


$$
\begin{aligned}
& \left\|f^{\left(\lambda, \beta_{1}, \beta_{2}\right)}\left(x_{1}, x_{2}\right)\right\|_{p} \lesssim\left\{\lambda_{1,1}^{\theta}\|f\|_{p}^{\theta}+\sum_{n_{1}=1}^{\infty}\left|\lambda_{n_{1}+1,1}^{\theta}-\lambda_{n_{1}, 1}^{\theta}\right| Y_{n_{1}, 0}^{\theta}(f)_{p}\right. \\
& +\sum_{n_{2}=1}^{\infty}\left|\lambda_{1, n_{2}+1}^{\theta}-\lambda_{1, n_{2}}^{\theta}\right| Y_{0, n_{2}}^{\theta}(f)_{p} \\
& \left.+\sum_{n_{1}=1}^{\infty} \sum_{n_{2}=1}^{\infty}\left|\lambda_{n_{1}, n_{2}}^{\theta}-\lambda_{n_{1}+1, n_{2}}^{\theta}-\lambda_{n_{1}, n_{2}+1}^{\theta}+\lambda_{n_{1}+1, n_{2}+1}^{\theta}\right| Y_{n_{1}, n_{2}}^{\theta}(f)_{p}\right\}^{\frac{1}{\theta}}, \\
& \left\|f^{\left(\lambda, \beta_{1}, \beta_{2}\right)}\left(x_{1}, x_{2}\right)\right\|_{p} \lesssim\left\{\lambda_{1,1}^{\theta}\|f\|_{p}^{\theta}+\sum_{\nu_{1}=1}^{\infty}\left|\lambda_{2^{\nu_{1}, 1}}^{\theta}-\lambda_{2^{\nu_{1}-1}, 1}^{\theta}\right| Y_{2^{\nu_{1}-1,0}}^{\theta}(f)_{p}\right. \\
& +\sum_{\nu_{2}=1}^{\infty}\left|\lambda_{1,2^{\nu_{2}}}^{\theta}-\lambda_{1,2^{\nu_{2}-1}}^{\theta}\right| Y_{0,2^{\nu_{2}-1}}^{\theta}(f)_{p} \\
& \left.+\sum_{\nu_{1}=1}^{\infty} \sum_{\nu_{2}=1}^{\infty}\left|\lambda_{2^{\nu_{1}}, 2^{\nu_{2}}}^{\theta}-\lambda_{2^{\nu_{1}-1}, 2^{\nu_{2}}}^{\theta}-\lambda_{2^{\nu_{1}}, 2^{\nu_{2}-1}}^{\theta}+\lambda_{2^{\nu_{1}-1}, 2^{\nu_{2}-1}}^{\theta}\right| Y_{2^{\nu_{1}}-1,2^{\nu_{2}}-1}^{\theta}(f)_{p}\right\}^{\frac{1}{\theta}}
\end{aligned}
$$

and

$$
\begin{aligned}
& Y_{2^{m_{1}-1,2^{m_{2}-1}}}\left(f^{\left(\lambda, \beta_{1}, \beta_{2}\right)}\left(x_{1}, x_{2}\right)\right)_{p} \lesssim\left\{\lambda_{2^{m_{1}-1}, 2^{m_{2}-1}}^{\theta} Y_{2^{m_{1}-1,2^{m_{2}-1}}}^{\theta}(f)_{p}\right. \\
& +\sum_{\nu_{1}=m_{1}}^{\infty}\left|\lambda_{2^{\nu_{1}}, 2^{m_{2}-1}}^{\theta}-\lambda_{2^{\nu_{1}-1}, 2^{m_{2}-1}}^{\theta}\right| Y_{2^{\nu_{1}}-1,2^{m_{2}-1}}^{\theta}(f)_{p} \\
& +\sum_{\nu_{2}=m_{2}}^{\infty}\left|\lambda_{2^{m_{1}-1}, 2^{\nu_{2}}}^{\theta}-\lambda_{2^{m_{1}-1}, 2^{\nu_{2}-1}}^{\theta}\right| Y_{2^{m_{1}-1,2^{\nu_{2}-1}}}^{\theta}(f)_{p} \\
& +\sum_{\nu_{1}=m_{1}}^{\infty} \sum_{\nu_{2}=m_{2}}^{\infty} \mid \lambda_{2^{\nu_{1}}, 2^{\nu_{2}}}^{\theta}-\lambda_{2^{\nu_{1}-1}, 2^{\nu_{2}}}^{\theta} \\
& \left.-\lambda_{2^{\nu_{1}}, 2^{\nu_{2}-1}}^{\theta}+\lambda_{2^{\nu_{1}-1}, 2^{\nu_{2}-1}}^{\theta} \mid Y_{2^{\nu_{1}}-1,2^{\nu_{2}-1}}^{\theta}(f)_{p}\right\}^{\frac{1}{\theta}} .
\end{aligned}
$$

Proof of Theorem 3.3.1. Let the series (3.3.4) be convergent and $f \in L_{p}^{0}\left(\mathbb{T}^{2}\right)$. We use the following inequality

$$
\begin{aligned}
\lambda_{2^{n_{1}-1}, 2^{n_{2}-1}}^{\theta} & =\lambda_{1,2^{n_{2}-1}}^{\theta}+\sum_{m_{1}=2}^{n_{1}}\left(\lambda_{2^{m_{1}-1}, 2^{n_{2}-1}}^{\theta}-\lambda_{2^{m_{1}-2}, 2^{n_{2}-1}}^{\theta}\right) \\
& =\lambda_{1,1}^{\theta}+\sum_{m_{2}=2}^{n_{2}}\left(\lambda_{1,2^{m_{2}-1}}^{\theta}-\lambda_{1,2^{m_{2}-2}}^{\theta}\right)+\sum_{m_{1}=2}^{n_{1}}\left(\lambda_{2^{m_{1}-1}, 1}^{\theta}-\lambda_{2^{m_{1}-2}, 1}^{\theta}\right)
\end{aligned}
$$




$$
\begin{aligned}
& +\sum_{m_{1}=2}^{n_{1}} \sum_{m_{2}=2}^{n_{2}}\left(\lambda_{2^{m_{1}-1}, 2^{m_{2}-1}}^{\theta}-\lambda_{2^{m_{1}-2}, 2^{m_{2}-1}}^{\theta}-\lambda_{2^{m_{1}-1}, 2^{m_{2}-2}}^{\theta}+\lambda_{2^{m_{1}-2}, 2^{m_{2}-2}}^{\theta}\right) \\
& \leqslant \lambda_{1,1}^{\theta}+\sum_{m_{2}=2}^{n_{2}}\left|\lambda_{1,2^{m_{2}-1}}^{\theta}-\lambda_{1,2^{m_{2}-2}}^{\theta}\right|+\sum_{m_{1}=2}^{n_{1}}\left|\lambda_{2^{m_{1}-1}, 1}^{\theta}-\lambda_{2^{m_{1}-2}, 1}^{\theta}\right| \\
& +\sum_{m_{1}=2}^{n_{1}} \sum_{m_{2}=2}^{n_{2}}\left|\lambda_{2^{m_{1}-1}, 2^{m_{2}-1}}^{\theta}-\lambda_{2^{m_{1}-2}, 2^{m_{2}-1}}^{\theta}-\lambda_{2^{m_{1}-1}, 2^{m_{2}-2}}^{\theta}+\lambda_{2^{m_{1}-2}, 2^{m_{2}-2}}^{\theta}\right| .
\end{aligned}
$$

Let us denote $\Delta_{n_{1}, n_{2}}=\sum_{\nu_{1}=2^{n_{1}-1}}^{2^{n_{1}}-1} \sum_{\nu_{2}=2^{n_{2}-1}}^{2^{n_{2}-1}} A_{\nu_{1}, \nu_{2}}\left(f, x_{1}, x_{2}\right) \quad\left(n_{1}, n_{2}=1,2, \ldots\right)$; see $(3.2 .3)$.

We will use several times the simple fact that, for any $\theta>0$, one has

$$
C_{1}(\theta)\left(j_{1}^{\theta}+j_{2}^{\theta}\right) \leqslant\left(j_{1}+j_{2}\right)^{\theta} \leqslant C_{2}(\theta)\left(j_{1}^{\theta}+j_{2}^{\theta}\right), \quad j_{1}, j_{2} \geqslant 0
$$

Using (3.3.5), we derive

$$
\begin{aligned}
& I_{1}:=\left\{\int_{0}^{2 \pi} \int_{0}^{2 \pi}\left[\sum_{n_{1}=1}^{\infty} \sum_{n_{2}=1}^{\infty} \lambda_{2^{n_{1}-1}, 2^{n_{2}-1}}^{2} \Delta_{n_{1}, n_{2}}^{2}\right]^{\frac{p}{2}} d x_{1} d x_{2}\right\}^{\frac{1}{p}} \\
& =\left\|\left[\sum_{n_{1}=1}^{\infty} \sum_{n_{2}=1}^{\infty} \lambda_{2^{n_{1}-1}, 2^{n_{2}-1}}^{2} \Delta_{n_{1}, n_{2}}^{2}\right]^{\frac{1}{2}}\right\|_{p} \\
& =\|\left[\lambda_{1,1}^{2} \Delta_{1,1}^{2}+\sum_{n_{1}=2}^{\infty} \lambda_{2^{n_{1}-1}, 1}^{2} \Delta_{n_{1}, 1}^{2}+\sum_{n_{2}=2}^{\infty} \lambda_{1,2^{n_{2}-1}}^{2} \Delta_{1, n_{2}}^{2}\right. \\
& \left.+\sum_{n_{1}=2}^{\infty} \sum_{n_{2}=2}^{\infty} \lambda_{2^{n_{1}-1}, 2^{n_{2}-1}}^{2} \Delta_{n_{1}, n_{2}}^{2}\right]^{\frac{1}{2}} \|_{p} \\
& \leqslant \|\left(\lambda_{1,1}^{2} \Delta_{1,1}^{2}+\sum_{n_{1}=2}^{\infty} \Delta_{n_{1}, 1}^{2}\left[\lambda_{1,1}^{\theta}+\sum_{\nu_{1}=2}^{n_{1}}\left|\lambda_{2^{\nu_{1}-1}, 1}^{\theta}-\lambda_{2^{\nu_{1}-2}, 1}^{\theta}\right|\right]^{\frac{2}{\theta}}\right. \\
& +\sum_{n_{2}=2}^{\infty} \Delta_{1, n_{2}}^{2}\left[\lambda_{1,1}^{\theta}+\sum_{\nu_{2}=2}^{n_{2}}\left|\lambda_{1,2^{\nu_{2}-1}}^{\theta}-\lambda_{1,2^{\nu_{2}-2}}^{\theta}\right|\right]^{\frac{2}{\theta}} \\
& +\sum_{n_{1}=2}^{\infty} \sum_{n_{2}=2}^{\infty} \Delta_{n_{1}, n_{2}}^{2}\left[\lambda_{1,1}^{\theta}+\sum_{\nu_{2}=2}^{n_{2}}\left|\lambda_{1,2^{\nu_{2}-1}}^{\theta}-\lambda_{1,2^{\nu_{2}-2}}^{\theta}\right|+\sum_{\nu_{1}=2}^{n_{1}}\left|\lambda_{2^{\nu_{1}-1}, 1}^{\theta}-\lambda_{2^{\nu_{1}-2}, 1}^{\theta}\right|\right. \\
& \left.\left.+\sum_{\nu_{1}=2}^{n_{1}} \sum_{\nu_{2}=2}^{n_{2}}\left|\lambda_{2^{\nu_{1}-1}, 2^{\nu_{2}-1}}^{\theta}-\lambda_{2^{\nu_{1}-2}, 2^{\nu_{2}-1}}^{\theta}-\lambda_{2^{\nu_{1}-1}, 2^{\nu_{2}-2}}^{\theta}+\lambda_{2^{\nu_{1}-2}, 2^{\nu_{2}-2}}^{\theta}\right|\right]^{\frac{2}{\theta}}\right)^{\frac{1}{2}} \|_{p}
\end{aligned}
$$




$$
\begin{aligned}
& \lesssim \|\left(\lambda_{1,1}^{2} \Delta_{1,1}^{2}+\lambda_{1,1}^{2} \sum_{n_{1}=2}^{\infty} \Delta_{n_{1}, 1}^{2}+\lambda_{1,1}^{2} \sum_{n_{2}=2}^{\infty} \Delta_{1, n_{2}}^{2}+\lambda_{1,1}^{2} \sum_{n_{1}=2}^{\infty} \sum_{n_{2}=2}^{\infty} \Delta_{n_{1}, n_{2}}^{2}\right. \\
& +\sum_{n_{1}=2}^{\infty} \Delta_{n_{1}, 1}^{2}\left[\sum_{\nu_{1}=2}^{n_{1}}\left|\lambda_{2^{\nu_{1}-1}, 1}^{\theta}-\lambda_{2^{\nu_{1}-2}, 1}^{\theta}\right|\right]^{\frac{2}{\theta}} \\
& +\sum_{n_{2}=2}^{\infty} \Delta_{1, n_{2}}^{2}\left[\sum_{\nu_{2}=2}^{n_{2}}\left|\lambda_{1,2^{\nu_{2}-1}}^{\theta}-\lambda_{1,2^{\nu_{2}-2}}^{\theta}\right|\right]^{\frac{2}{\theta}} \\
& +\sum_{n_{1}=2}^{\infty} \sum_{n_{2}=2}^{\infty} \Delta_{n_{1}, n_{2}}^{2}\left[\sum_{\nu_{2}=2}^{n_{2}}\left|\lambda_{1,2^{\nu_{2}-1}}^{\theta}-\lambda_{1,2^{\nu_{2}-2}}^{\theta}\right|+\sum_{\nu_{1}=2}^{n_{1}}\left|\lambda_{2^{\nu_{1}-1}, 1}^{\theta}-\lambda_{2^{\nu_{1}-2}, 1}^{\theta}\right|\right. \\
& \left.\left.+\sum_{n_{1}}^{n_{2}} \sum_{\nu_{2}=2}^{\theta} \mid \lambda_{2^{\nu_{1}-1}, 2^{\nu_{2}-1}}^{\theta}-\lambda_{2^{\nu_{1}-2}, 2^{\nu_{2}-1}}^{\theta}-\lambda_{2^{\nu_{1}-1}, 2^{\nu_{2}-2}}^{\theta}+\lambda_{2^{\nu_{1}-2}, 2^{\nu_{2}-2} \mid}^{\theta}\right]^{\frac{2}{\theta}}\right)^{\frac{1}{2}} \|_{p} .
\end{aligned}
$$

This implies that

$$
\begin{aligned}
& I_{1} \lesssim \|\left\{\lambda_{1,1}^{2}\left(\Delta_{1,1}^{2}+\sum_{n_{1}=2}^{\infty} \Delta_{n_{1}, 1}^{2}+\sum_{n_{2}=2}^{\infty} \Delta_{1, n_{2}}^{2}+\sum_{n_{1}=2}^{\infty} \sum_{n_{2}=2}^{\infty} \Delta_{n_{1}, n_{2}}^{2}\right)\right. \\
& +\sum_{n_{1}=2}^{\infty} \Delta_{n_{1}, 1}^{2}\left[\sum_{\nu_{1}=2}^{n_{1}}\left|\lambda_{2^{\nu_{1}-1}, 1}^{\theta}-\lambda_{2^{\nu_{1}-2}, 1}^{\theta}\right|\right]^{\frac{2}{\theta}} \\
& +\sum_{n_{1}=2}^{\infty} \sum_{n_{2}=2}^{\infty} \Delta_{n_{1}, n_{2}}^{2}\left[\sum_{\nu_{1}=2}^{n_{1}}\left|\lambda_{2^{\nu_{1}-1}, 1}^{\theta}-\lambda_{2^{\nu_{1}-2}, 1}^{\theta}\right|\right]^{\frac{2}{\theta}} \\
& +\sum_{n_{2}=2}^{\infty} \Delta_{1, n_{2}}^{2}\left[\sum_{\nu_{2}=2}^{n_{2}}\left|\lambda_{1,2^{\nu_{2}-1}}^{\theta}-\lambda_{1,2^{\nu_{2}-2}}^{\theta}\right|\right]^{\frac{2}{\theta}} \\
& +\sum_{n_{1}=2}^{\infty} \sum_{n_{2}=2}^{\infty} \Delta_{n_{1}, n_{2}}^{2}\left[\sum_{\nu_{2}=2}^{n_{2}}\left|\lambda_{1,2^{\nu_{2}-1}}^{\theta}-\lambda_{1,2^{\nu_{2}-2}}^{\theta}\right|\right]^{\frac{2}{\theta}} \\
& +\sum_{n_{1}=2}^{\infty} \sum_{n_{2}=2}^{\infty} \Delta_{n_{1}, n_{2}}^{2}\left[\sum_{\nu_{1}=2}^{n_{1}} \sum_{\nu_{2}=2}^{n_{2}} \mid \lambda_{2^{\nu_{1}-1}, 2^{\nu_{2}-1}}^{\theta}\right. \\
& \left.\left.-\lambda_{2^{\nu_{1}-2}, 2^{\nu_{2}-1}}^{\theta}-\lambda_{2^{\nu_{1}-1}, 2^{\nu_{2}-2}}^{\theta}+\lambda_{2^{\nu_{1}-2}, 2^{\nu_{2}-2}}^{\theta} \mid\right]^{\frac{2}{\theta}}\right\}^{\frac{1}{2}} \|_{p} \\
& \lesssim \lambda_{1,1}\left\|\left[\sum_{n_{1}=1}^{\infty} \sum_{n_{2}=1}^{\infty} \Delta_{n_{1}, n_{2}}^{2}\right]^{\frac{1}{2}}\right\|_{p}
\end{aligned}
$$




$$
\begin{aligned}
& +\left\|\left(\sum_{n_{1}=2}^{\infty} \sum_{n_{2}=1}^{\infty} \Delta_{n_{1}, n_{2}}^{2}\left[\sum_{\nu_{1}=2}^{n_{1}}\left|\lambda_{2^{\nu_{1}-1}, 1}^{\theta}-\lambda_{2^{\nu_{1}-2}, 1}^{\theta}\right|\right]^{\frac{2}{\theta}}\right)^{\frac{1}{2}}\right\|_{p} \\
& +\left\|\left(\sum_{n_{1}=1}^{\infty} \sum_{n_{2}=2}^{\infty} \Delta_{n_{1}, n_{2}}^{2}\left[\sum_{\nu_{2}=2}^{n_{2}}\left|\lambda_{1,2^{\nu_{2}-1}}^{\theta}-\lambda_{1,2^{\nu_{2}-2}}^{\theta}\right|\right]^{\frac{2}{\theta}}\right)^{\frac{1}{2}}\right\|_{p} \\
& +\left\|\left(\sum_{n_{1}=2}^{\infty} \sum_{n_{2}=2}^{\infty} \Delta_{n_{1}, n_{2}}^{2}\left[\sum_{\nu_{1}=2}^{n_{1}} \sum_{\nu_{2}=2}^{n_{2}} \mid \lambda_{2^{\nu_{1}-1}, 2^{\nu_{2}-1}}^{\theta}-\lambda_{2^{\nu_{1}-2}, 2^{\nu_{2}-1}}^{\theta}-\lambda_{2^{\nu_{1}-1}, 2^{\nu_{2}-2}}^{\theta}+\lambda_{2^{\nu_{1}-2}, 2^{\nu_{2}-2}}^{\theta}\right]^{\frac{2}{\theta}}\right)^{\frac{1}{2}}\right\|_{p} \\
& =: J_{1}+J_{2}+J_{3}+J_{4} .
\end{aligned}
$$

Let us estimate $J_{1}$. Applying Lemma 3.2.5, we have $J_{1} \leqslant C \lambda_{11}\|f\|_{p}<\infty$. Now we estimate $J_{2}$ given by

$$
J_{2}=\left\{\int_{0}^{2 \pi} \int_{0}^{2 \pi}\left[\sum_{n_{1}=2}^{\infty} \sum_{n_{2}=1}^{\infty} \Delta_{n_{1}, n_{2}}^{2}\left[\sum_{\nu_{1}=2}^{n_{1}}\left|\lambda_{2^{\nu_{1}-1}, 1}^{\theta}-\lambda_{2^{\nu_{1}-2}, 1}^{\theta}\right|\right]^{\frac{2}{\theta}}\right]^{\frac{p}{2}} d x_{1} d x_{2}\right\}^{\frac{1}{p}}
$$

Using Minkowski's inequality and Lemma 1.5.5 (a) for $\frac{2}{\theta} \geqslant 1$, we derive

$$
\begin{aligned}
& \sum_{n_{2}=1}^{\infty} \sum_{n_{1}=2}^{\infty} \Delta_{n_{1}, n_{2}}^{2}\left[\sum_{\nu_{1}=2}^{n_{1}}\left|\lambda_{2^{\nu_{1}-1}, 1}^{\theta}-\lambda_{2^{\nu_{1}-2}, 1}^{\theta}\right|\right]^{\frac{2}{\theta}} \\
& \left.=\sum_{n_{1}=2}^{\infty}\left(\sum_{n_{2}=1}^{\infty}\left[\sum_{\nu_{1}=2}^{n_{1}}\left|\Delta_{n_{1}, n_{2}}\right| \theta\left|\lambda_{2^{\nu_{1}-1}, 1}^{\theta}-\lambda_{2^{\nu_{1}-2}, 1}^{\theta}\right|\right]^{\frac{2}{\theta}}\right)^{\frac{\theta}{2}}\right)^{\frac{2}{\theta}} \\
& \leqslant \sum_{n_{1}=2}^{\infty}\left(\sum_{\nu_{1}=2}^{n_{1}}\left[\sum_{n_{2}=1}^{\infty}\left|\Delta_{n_{1}, n_{2}}\right|^{2}\left|\lambda_{2^{\nu_{1}-1}, 1}^{\theta}-\lambda_{2^{\nu_{1}-2}, 1}^{\theta}\right|^{\frac{2}{\theta}}\right]^{\frac{\theta}{2}}\right)^{\frac{2}{\theta}} \\
& =\left(\sum_{n_{1}=2}^{\infty}\left\{\sum_{\nu_{1}=2}^{n_{1}}\left[\sum_{n_{2}=1}^{\infty}\left|\Delta_{n_{1}, n_{2}}\right|^{2}\left|\lambda_{2^{\nu_{1}-1}, 1}^{\theta}-\lambda_{2^{\nu_{1}-2}, 1}^{\theta}\right|^{\frac{2}{\theta}}\right\}^{\frac{\theta}{2}}\right)^{\frac{2}{\theta}}\right)^{\frac{\theta}{\theta}} \\
& \leqslant\left(\sum_{\nu_{1}=2}^{\infty}\left\{\sum_{n_{1}=\nu_{1}}^{\infty} \sum_{n_{2}=1}^{\infty}\left|\Delta_{n_{1}, n_{2}}\right|^{2}\left|\lambda_{2^{\nu_{1}-1}, 1}^{\theta}-\lambda_{2^{\nu_{1}-2}, 1}^{\theta}\right|^{\frac{2}{\theta}}\right\}^{\frac{\theta}{2}}\right)^{\frac{2}{\theta}} \\
& =\left(\sum_{\nu_{1}=2}^{\infty}\left|\lambda_{2^{\nu_{1}-1}, 1}^{\theta}-\lambda_{2^{\nu_{1}-2}, 1}^{\theta}\right|\left(\sum_{n_{1}=\nu_{1}}^{\infty} \sum_{n_{2}=1}^{\infty}\left|\Delta_{n_{1}, n_{2}}\right|^{2}\right)^{\frac{\theta}{2}}\right)^{\frac{2}{\theta}} \cdot
\end{aligned}
$$


Applying this estimate, we obtain

$$
\begin{aligned}
J_{2} \leqslant & \left\{\int_{0}^{2 \pi} \int_{0}^{2 \pi}\left[\left\{\sum_{\nu_{1}=2}^{\infty}\left|\lambda_{2^{\nu_{1}-1}, 1}^{\theta}-\lambda_{2^{\nu_{1}-2}, 1}^{\theta}\right|\left(\sum_{n_{1}=\nu_{1}}^{\infty} \sum_{n_{2}=1}^{\infty}\left|\Delta_{n_{1}, n_{2}}\right|^{2}\right)^{\frac{\theta}{2}}\right\}^{\frac{2}{\theta}}\right]^{\frac{p}{2}} d x_{1} d x_{2}\right\}^{\frac{1}{p}} \\
& =\left(\left\{\int_{0}^{2 \pi} \int_{0}^{2 \pi}\left(\sum_{\nu_{1}=2}^{\infty}\left|\lambda_{2^{\nu_{1}-1}, 1}^{\theta}-\lambda_{2^{\nu_{1}-2}, 1}^{\theta}\right|\left(\sum_{n_{1}=\nu_{1}}^{\infty} \sum_{n_{2}=1}^{\infty}\left|\Delta_{n_{1}, n_{2}}\right|^{2}\right)^{\frac{\theta}{2}}\right)^{\frac{p}{\theta}} d x_{1} d x_{2}\right\}^{\frac{\theta}{p}}\right)^{\frac{1}{\theta}} .
\end{aligned}
$$

Further, using Minkowski's inequality for $\frac{p}{\theta} \geqslant 1$, Lemmas 3.2.4 and 3.2.5, we have

$$
\begin{aligned}
J_{2} & \leqslant\left(\sum_{\nu_{1}=2}^{\infty}\left|\lambda_{2^{\nu_{1}-1}, 1}^{\theta}-\lambda_{2^{\nu_{1}-2}, 1}^{\theta}\right|\left\{\int_{0}^{2 \pi} \int_{0}^{2 \pi}\left(\left(\sum_{n_{1}=\nu_{1}}^{\infty} \sum_{n_{2}=1}^{\infty}\left|\Delta_{n_{1}, n_{2}}\right|^{2}\right)^{\frac{\theta}{2}}\right)^{\frac{p}{\theta}} d x_{1} d x_{2}\right\}^{\frac{\theta}{p}}\right)^{\frac{1}{\theta}} \\
& =\left(\sum_{\nu_{1}=2}^{\infty}\left|\lambda_{2^{\nu_{1}-1}, 1}^{\theta}-\lambda_{2^{\nu_{1}-2}, 1}^{\theta}\right|||\left(\sum_{n_{1}=\nu_{1}}^{\infty} \sum_{n_{2}=1}^{\infty}\left|\Delta_{n_{1}, n_{2}}\right|^{2}\right)^{\frac{1}{2}}||_{p}^{\theta}\right)^{\frac{1}{\theta}} \\
& \lesssim\left(\sum_{\nu_{1}=1}^{\infty}\left|\lambda_{2^{\nu_{1}}, 1}^{\theta}-\lambda_{2^{\nu_{1}-1}, 1}^{\theta}\right| Y_{2^{\nu_{1}}-1,0}^{\theta}(f)_{p}\right)^{\frac{1}{\theta}} .
\end{aligned}
$$

Thus, we obtain

$$
J_{2} \lesssim\left(\sum_{\nu_{1}=1}^{\infty}\left|\lambda_{2^{\nu_{1}, 1}}^{\theta}-\lambda_{2^{\nu_{1}-1}, 1}^{\theta}\right| Y_{2^{\nu_{1}}-1,0}^{\theta}(f)_{p}\right)^{\frac{1}{\theta}} .
$$

From (3.3.4), it follows that $J_{2}<\infty$.

Let us estimate

$$
J_{3}=\left\{\int_{0}^{2 \pi} \int_{0}^{2 \pi}\left[\sum_{n_{1}=1}^{\infty} \sum_{n_{2}=2}^{\infty} \Delta_{n_{1}, n_{2}}^{2}\left[\sum_{\nu_{2}=2}^{n_{2}}\left|\lambda_{1,2^{\nu_{2}-1}}^{\theta}-\lambda_{1,2^{\nu_{2}-2}}^{\theta}\right|\right]^{\frac{2}{\theta}}\right]^{\frac{p}{2}} d x_{1} d x_{2}\right\}^{\frac{1}{p}} .
$$

We use Lemmas 1.5.6 and 1.5.5 (a) with $\frac{2}{\theta} \geqslant 1$ to get

$$
\begin{aligned}
& \sum_{n_{1}=1}^{\infty} \sum_{n_{2}=2}^{\infty} \Delta_{n_{1}, n_{2}}^{2}\left[\sum_{\nu_{2}=2}^{n_{2}}\left|\lambda_{1,2^{\nu_{2}-1}}^{\theta}-\lambda_{1,2^{\nu_{2}-2}}^{\theta}\right|\right]^{\frac{2}{\theta}} \\
& \leqslant \sum_{n_{2}=2}^{\infty}\left(\sum_{\nu_{2}=2}^{n_{2}}\left(\sum_{n_{1}=1}^{\infty}\left[\left.\left|\Delta_{n_{1}, n_{2}}\right|\right|^{\theta}\left|\lambda_{1,2^{\nu_{2}-1}}^{\theta}-\lambda_{1,2^{\nu_{2}-2}}^{\theta}\right|\right]^{\frac{2}{\theta}}\right)^{\frac{\theta}{2}}\right)^{\frac{2}{\theta}}
\end{aligned}
$$




$$
\begin{aligned}
& =\left(\left[\sum_{n_{2}=2}^{\infty}\left\{\sum_{\nu_{2}=2}^{n_{2}}\left(\sum_{n_{1}=1}^{\infty}\left|\Delta_{n_{1}, n_{2}}\right|^{2}\left|\lambda_{1,2^{\nu_{2}-1}}^{\theta}-\lambda_{1,2^{\nu_{2}-2}}^{\theta}\right|^{\frac{2}{\theta}}\right)^{\frac{\theta}{2}}\right\}^{\frac{2}{\theta}}\right]^{\frac{\theta}{2}}\right)^{\frac{2}{\theta}} \\
& \leqslant\left(\sum_{\nu_{2}=2}^{\infty}\left|\lambda_{1,2^{\nu_{2}-1}}^{\theta}-\lambda_{1,2^{\nu_{2}-2}}^{\theta}\right|\left(\sum_{n_{2}=\nu_{2}}^{\infty} \sum_{n_{1}=1}^{\infty}\left|\Delta_{n_{1}, n_{2}}\right|^{2}\right)^{\frac{\theta}{2}}\right)^{\frac{2}{\theta}} .
\end{aligned}
$$

Using this and Lemma 1.5.6 with $\frac{p}{\theta} \geqslant 1$, we obtain that

$$
\begin{aligned}
J_{3} & =\left\{\int_{0}^{2 \pi} \int_{0}^{2 \pi}\left[\sum_{n_{1}=1}^{\infty} \sum_{n_{2}=2}^{\infty} \Delta_{n_{1}, n_{2}}^{2}\left[\sum_{\nu_{2}=2}^{n_{2}}\left|\lambda_{1,2^{\nu_{2}-1}}^{\theta}-\lambda_{1,2^{\nu_{2}-2}}^{\theta}\right|\right]^{\frac{2}{\theta}}\right]^{\frac{p}{2}} d x_{1} d x_{2}\right\}^{\frac{1}{p}} \\
& \leqslant\left\{\int_{0}^{2 \pi} \int_{0}^{2 \pi}\left[\left(\sum_{\nu_{2}=2}^{\infty}\left|\lambda_{1,2^{\nu_{2}-1}}^{\theta}-\lambda_{1,2^{\nu_{2}-2}}^{\theta}\right|\left(\sum_{n_{2}=\nu_{2}}^{\infty} \sum_{n_{1}=1}^{\infty}\left|\Delta_{n_{1}, n_{2}}\right|^{2}\right)^{\frac{\theta}{2}}\right)^{\frac{2}{\theta}}\right]^{\frac{p}{2}} d x_{1} d x_{2}\right\}^{\frac{1}{p}} \\
& \leqslant\left(\sum_{\nu_{2}=2}^{\infty}\left|\lambda_{1,2^{\nu_{2}-1}}^{\theta}-\lambda_{1,2^{\nu_{2}-2}}^{\theta}\right|\left\{\int_{0}^{2 \pi} \int_{0}^{2 \pi}\left(\sum_{n_{2}=\nu_{2}}^{\infty} \sum_{n_{1}=1}^{\infty}\left|\Delta_{n_{1}, n_{2}}\right|^{2}\right)^{\frac{p}{2}} d x_{1} d x_{2}\right\}^{\frac{\theta}{p}}\right)^{\frac{1}{\theta}} .
\end{aligned}
$$

By Lemmas 3.2.4 and 3.2.5, we have

$$
J_{3} \lesssim\left(\sum_{\nu_{2}=1}^{\infty}\left|\lambda_{1,2^{\nu_{2}}}^{\theta}-\lambda_{1,2^{\nu_{2}-1}}^{\theta}\right| Y_{0,2^{\nu_{2}-1}}^{\theta}(f)_{p}\right)^{\frac{1}{\theta}}<\infty
$$

provided that (3.3.4) holds.

To estimate $J_{4}$, we first obtain the upper estimate of the following sum. Applying Lemmas 1.5.5 and 1.5.6 twice for $\frac{2}{\theta} \geqslant 1$, we get

$$
\begin{aligned}
& \sum_{n_{1}=2}^{\infty} \sum_{n_{2}=2}^{\infty} \Delta_{n_{1}, n_{2}}^{2}\left[\sum_{\nu_{1}=2}^{n_{1}} \sum_{\nu_{2}=2}^{n_{2}}\left|\lambda_{2^{\nu_{1}-1}, 2^{\nu_{2}-1}}^{\theta}-\lambda_{2^{\nu_{1}-2}, 2^{\nu_{2}-1}}^{\theta}-\lambda_{2^{\nu_{1}-1}, 2^{\nu_{2}-2}}^{\theta}+\lambda_{2^{\nu_{1}-2}, 2^{\nu_{2}-2}}^{\theta}\right|\right]^{\frac{2}{\theta}} \\
& \leqslant \sum_{n_{1}=2}^{\infty}\left(\sum _ { \nu _ { 2 } = 2 } ^ { \infty } \left\{\sum _ { n _ { 2 } = \nu _ { 2 } } ^ { \infty } \left[\left(\sum_{\nu_{1}=2}^{n_{1}}\left|\Delta_{n_{1}, n_{2}}\right|^{\theta} \mid \lambda_{2^{\nu_{1}-1}, 2^{\nu_{2}-1}}^{\theta}\right.\right.\right.\right. \\
& \left.\left.\left.\left.-\lambda_{2^{\nu_{1}-2}, 2^{\nu_{2}-1}}^{\theta}-\lambda_{2^{\nu_{1}-1}, 2^{\nu_{2}-2}}^{\theta}+\lambda_{2^{\nu_{1}-2}, 2^{\nu_{2}-2}}^{\theta} \mid\right)\right]^{\frac{2}{\theta}}\right\}^{\frac{\theta}{2}}\right)^{\frac{2}{\theta}} \\
& \leqslant \sum_{n_{1}=2}^{\infty}\left(\sum _ { \nu _ { 2 } = 2 } ^ { \infty } \sum _ { \nu _ { 1 } = 2 } ^ { n _ { 1 } } \left\{\sum _ { n _ { 2 } = \nu _ { 2 } } ^ { \infty } \left[\left|\Delta_{n_{1}, n_{2}}\right|^{\theta} \mid \lambda_{2^{\nu_{1}-1}, 2^{\nu_{2}-1}}^{\theta}\right.\right.\right. \\
& \left.\left.\left.-\lambda_{2^{\nu_{1}-2}, 2^{\nu_{2}-1}}^{\theta}-\lambda_{2^{\nu_{1}-1}, 2^{\nu_{2}-2}}^{\theta}+\lambda_{2^{\nu_{1}-2}, 2^{\nu_{2}-2}}^{\theta} \mid\right]^{\frac{2}{\theta}}\right\}^{\frac{\theta}{2}}\right)^{\frac{2}{\theta}}
\end{aligned}
$$




$$
\begin{aligned}
& =\left(\left(\sum _ { n _ { 1 } = 2 } ^ { \infty } \left\{\sum _ { \nu _ { 2 } = 2 } ^ { \infty } \sum _ { \nu _ { 1 } = 2 } ^ { n _ { 1 } } \left[\sum_{n_{2}=\nu_{2}}^{\infty}\left|\Delta_{n_{1}, n_{2}}\right|^{2} \mid \lambda_{2^{\nu_{1}-1}, 2^{\nu_{2}-1}}^{\theta}\right.\right.\right.\right. \\
& \left.\left.\left.\left.-\lambda_{2^{\nu_{1}-2}, 2^{\nu_{2}-1}}^{\theta}-\lambda_{2^{\nu_{1}-1}, 2^{\nu_{2}-2}}^{\theta}+\left.\lambda_{2^{\nu_{1}-2}, 2^{\nu_{2}-2}}^{\theta}\right|^{\frac{2}{\theta}}\right]^{\frac{\theta}{2}}\right\}^{\frac{2}{\theta}}\right)^{\frac{\theta}{2}}\right)^{\frac{2}{\theta}} \\
& \leqslant\left(\sum _ { \nu _ { 2 } = 2 } ^ { \infty } \left\{\sum _ { n _ { 1 } = 2 } ^ { \infty } \left[\sum _ { \nu _ { 1 } = 2 } ^ { n _ { 1 } } \left(\sum_{n_{2}=\nu_{2}}^{\infty}\left|\Delta_{n_{1}, n_{2}}\right|^{2} \mid \lambda_{2^{\nu_{1}-1}, 2^{\nu_{2}-1}}^{\theta}\right.\right.\right.\right. \\
& \left.\left.\left.\left.-\lambda_{2^{\nu_{1}-2}, 2^{\nu_{2}-1}}^{\theta}-\lambda_{2^{\nu_{1}-1}, 2^{\nu_{2}-2}}^{\theta}+\left.\lambda_{2^{\nu_{1}-2}, 2^{\nu_{2}-2}}^{\theta}\right|^{\frac{2}{\theta}}\right)^{\frac{\theta}{2}}\right]^{\frac{2}{\theta}}\right\}^{\frac{\theta}{2}}\right)^{\frac{2}{\theta}} \\
& \leqslant\left(\sum _ { \nu _ { 2 } = 2 } ^ { \infty } \sum _ { \nu _ { 1 } = 2 } ^ { \infty } \left[\sum_{n_{1}=\nu_{1}}^{\infty} \sum_{n_{2}=\nu_{2}}^{\infty}\left|\Delta_{n_{1}, n_{2}}\right|^{2} \mid \lambda_{2^{\nu_{1}-1}, 2^{\nu_{2}-1}}^{\theta}\right.\right. \\
& \left.\left.-\lambda_{2^{\nu_{1}-2}, 2^{\nu_{2}-1}}^{\theta}-\lambda_{2^{\nu_{1}-1}, 2^{\nu_{2}-2}}^{\theta}+\left.\lambda_{2^{\nu_{1}-2}, 2^{\nu_{2}-2}}^{\theta}\right|^{\frac{2}{\theta}}\right]^{\frac{\theta}{2}}\right)^{\frac{2}{\theta}} \\
& =\left(\sum_{\nu_{2}=2}^{\infty} \sum_{\nu_{1}=2}^{\infty} \mid \lambda_{2^{\nu_{1}-1}, 2^{\nu_{2}-1}}^{\theta}-\lambda_{2^{\nu_{1}-2}, 2^{\nu_{2}-1}}^{\theta}\right. \\
& \left.-\lambda_{2^{\nu_{1}-1}, 2^{\nu_{2}-2}}^{\theta}+\lambda_{2^{\nu_{1}-2}, 2^{\nu_{2}-2}}^{\theta} \mid\left(\sum_{n_{1}=\nu_{1}}^{\infty} \sum_{n_{2}=\nu_{2}}^{\infty}\left|\Delta_{n_{1}, n_{2}}\right|^{2}\right)^{\frac{\theta}{2}}\right)^{\frac{2}{\theta}} .
\end{aligned}
$$

Hence, Lemma 1.5.6 with $\frac{p}{\theta} \geqslant 1$ implies that

$$
\begin{aligned}
& J_{4}=\left\{\int _ { 0 } ^ { 2 \pi } \int _ { 0 } ^ { 2 \pi } \left[\sum _ { n _ { 1 } = 2 } ^ { \infty } \sum _ { n _ { 2 } = 2 } ^ { \infty } \Delta _ { n _ { 1 } , n _ { 2 } } ^ { 2 } \left[\sum_{\nu_{1}=2}^{n_{1}} \sum_{\nu_{2}=2}^{n_{2}} \mid \lambda_{2^{\nu_{1}-1}, 2^{\nu_{2}-1}}^{\theta}-\lambda_{2^{\nu_{1}-2}, 2^{\nu_{2}-1}}^{\theta}-\right.\right.\right. \\
& \left.\left.\left.-\lambda_{2^{\nu_{1}-1}, 2^{\nu_{2}-2}}^{\theta}+\lambda_{2^{\nu_{1}-2}, 2^{\nu_{2}-2}}^{\theta} \mid\right]^{\frac{2}{\theta}}\right]^{\frac{p}{2}} d x_{1} d x_{2}\right\}^{\frac{1}{p}} \\
& \leqslant\left\{\int _ { 0 } ^ { 2 \pi } \int _ { 0 } ^ { 2 \pi } \left[\left(\sum_{\nu_{2}=2}^{\infty} \sum_{\nu_{1}=2}^{\infty} \mid \lambda_{2^{\nu_{1}-1}, 2^{\nu_{2}-1}}^{\theta}-\lambda_{2^{\nu_{1}-2}, 2^{\nu_{2}-1}}^{\theta}\right.\right.\right. \\
& \left.\left.\left.-\lambda_{2^{\nu_{1}-1}, 2^{\nu_{2}-2}}^{\theta}+\lambda_{2^{\nu_{1}-2}, 2^{\nu_{2}-2}}^{\theta} \mid\left(\sum_{n_{1}=\nu_{1}}^{\infty} \sum_{n_{2}=\nu_{2}}^{\infty}\left|\Delta_{n_{1}, n_{2}}\right|^{2}\right)^{\frac{\theta}{2}}\right)^{\frac{2}{\theta}}\right]^{\frac{p}{2}} d x_{1} d x_{2}\right\}^{\frac{1}{p}} \\
& =\left(\left\{\int _ { 0 } ^ { 2 \pi } \int _ { 0 } ^ { 2 \pi } \left[\sum_{\nu_{2}=2}^{\infty} \sum_{\nu_{1}=2}^{\infty} \mid \lambda_{2^{\nu_{1}-1}, 2^{\nu_{2}-1}}^{\theta}-\lambda_{2^{\nu_{1}-2}, 2^{\nu_{2}-1}}^{\theta}\right.\right.\right. \\
& \left.\left.\left.-\lambda_{2^{\nu_{1}-1}, 2^{\nu_{2}-2}}^{\theta}+\lambda_{2^{\nu_{1}-2}, 2^{\nu_{2}-2}}^{\theta} \mid\left(\sum_{n_{1}=\nu_{1}}^{\infty} \sum_{n_{2}=\nu_{2}}^{\infty}\left|\Delta_{n_{1}, n_{2}}\right|^{2}\right)^{\frac{\theta}{2}}\right]^{\frac{p}{\theta}} d x_{1} d x_{2}\right\}^{\frac{\theta}{p}}\right)^{\frac{1}{\theta}}
\end{aligned}
$$




$$
\begin{aligned}
& \leqslant\left(\sum_{\nu_{2}=2}^{\infty} \sum_{\nu_{1}=2}^{\infty} \mid \lambda_{2^{\nu_{1}-1}, 2^{\nu_{2}-1}}^{\theta}-\lambda_{2^{\nu_{1}-2}, 2^{\nu_{2}-1}}^{\theta}-\lambda_{2^{\nu_{1}-1}, 2^{\nu_{2}-2}}^{\theta}\right. \\
& \left.+\lambda_{2^{\nu_{1}-2}, 2^{\nu_{2}-2} \mid}^{\theta} \mid\left\{\int_{0}^{2 \pi} \int_{0}^{2 \pi}\left[\left(\sum_{n_{1}=\nu_{1}}^{\infty} \sum_{n_{2}=\nu_{2}}^{\infty}\left|\Delta_{n_{1}, n_{2}}\right|^{2}\right)^{\frac{\theta}{2}}\right]^{\frac{p}{\theta}} d x_{1} d x_{2}\right\}^{\frac{\theta}{p}}\right)^{\frac{1}{\theta}} \\
& =\left(\sum_{\nu_{2}=2}^{\infty} \sum_{\nu_{1}=2}^{\infty} \mid \lambda_{2^{\nu_{1}-1}, 2^{\nu_{2}-1}}^{\theta}-\lambda_{2^{\nu_{1}-2}, 2^{\nu_{2}-1}-\lambda_{2^{\nu_{1}-1}, 2^{\nu_{2}-2}}^{\theta}}^{\theta}\right. \\
& \left.+\lambda_{2^{\nu_{1}-2}, 2^{\nu_{2}-2} \mid}^{\theta}\left\{\int_{0}^{2 \pi} \int_{0}^{2 \pi}\left[\sum_{n_{1}=\nu_{1}}^{\infty} \sum_{n_{2}=\nu_{2}}^{\infty}\left|\Delta_{n_{1}, n_{2}}\right|^{2}\right]^{\frac{p}{2}} d x_{1} d x_{2}\right\}^{\frac{\theta}{p}}\right)^{\frac{1}{\theta}} .
\end{aligned}
$$

By Lemmas 3.2.4 and 3.2.5, we obtain

$$
J_{4} \lesssim\left(\sum_{\nu_{2}=1}^{\infty} \sum_{\nu_{1}=1}^{\infty}\left|\lambda_{2^{\nu_{1}}, 2^{\nu_{2}}}^{\theta}-\lambda_{2^{\nu_{1}-1}, 2^{\nu_{2}}}^{\theta}-\lambda_{2^{\nu_{1}}, 2^{\nu_{2}-1}}^{\theta}+\lambda_{2^{\nu_{1}-1}, 2^{\nu_{2}-1}}^{\theta}\right| Y_{2^{\nu_{1}-1,2^{\nu_{2}}-1}}^{\theta}(f)_{p}\right)^{\frac{1}{\theta}}
$$

By (3.3.4), we have $J_{4}<\infty$.

Collecting estimates of $J_{1}, J_{2}, J_{3}$ and $J_{4}$, we get $I_{1}<\infty$. Hence, by Lemma 3.2.5 (b), there exists a function $g\left(x_{1}, x_{2}\right) \in L_{p}^{0}$, with the Fourier series

$$
\sum_{n_{1}=1}^{\infty} \sum_{n_{2}=1}^{\infty} \lambda_{2^{n_{1}-1}, 2^{n_{2}-1}} \Delta_{n_{1}, n_{2}}
$$

and

$$
\|g\|_{p} \leqslant C(p) I_{1}
$$

We rewrite series (3.3.6) in the form of

$$
\sum_{n_{1}=1}^{\infty} \sum_{n_{2}=1}^{\infty} \gamma_{n_{1}, n_{2}} A_{n_{1} n_{2}}\left(x_{1}, x_{2}\right)
$$

where $\left(n_{1}, n_{2}=2,3, \ldots\right)$

$$
\begin{aligned}
& \gamma_{1,1}=\lambda_{1,1}, \quad \gamma_{1, \nu_{2}}=\lambda_{1,2^{n_{2}-1}} \text { for } 2^{n_{2}-1} \leqslant \nu_{2} \leqslant 2^{n_{2}}-1, \\
& \gamma_{\nu_{1}, 1}=\lambda_{2^{n_{1}-1}, 1} \text { for } 2^{n_{1}-1} \leqslant \nu_{1} \leqslant 2^{n_{1}}-1, \\
& \gamma_{\nu_{1}, \nu_{2}}=\lambda_{2^{n_{1}-1}, 2^{n_{2}-1}} \text { for } 2^{n_{2}-1} \leqslant \nu_{2} \leqslant 2^{n_{2}}-1,2^{n_{1}-1} \leqslant \nu_{1} \leqslant 2^{n_{1}}-1 .
\end{aligned}
$$


Now we consider the following series

$$
\sum_{n_{1}=1}^{\infty} \sum_{n_{2}=1}^{\infty} \lambda_{n_{1}, n_{2}} A_{n_{1} n_{2}}\left(x_{1}, x_{2}\right)=\sum_{n_{1}=1}^{\infty} \sum_{n_{2}=1}^{\infty} \gamma_{n_{1}, n_{2}} \Lambda_{n_{1}, n_{2}} A_{n_{1} n_{2}}\left(x_{1}, x_{2}\right),
$$

where

$$
\begin{aligned}
& \Lambda_{1,1}=1, \Lambda_{1, \nu_{2}}=\frac{\lambda_{1, \nu_{2}}}{\gamma_{1, \nu_{2}}}=\frac{\lambda_{1, \nu_{2}}}{\lambda_{1,2^{n_{2}-1}}} \text { for } 2^{n_{2}-1} \leqslant \nu_{2} \leqslant 2^{n_{2}}-1 \quad\left(n_{2}=2,\right. \\
& \Lambda_{\nu_{1}, 1}=\frac{\lambda_{\nu_{1}, 1}}{\gamma_{\nu_{1}, 1}}=\frac{\lambda_{\nu_{1}, 1}}{\lambda_{2^{n_{1}-1}, 1}} \text { for } 2^{n_{1}-1} \leqslant \nu_{1} \leqslant 2^{n_{1}}-1\left(n_{1}=2,3, \ldots\right), \\
& \Lambda_{\nu_{1}, \nu_{2}}=\frac{\lambda_{\nu_{1}, \nu_{2}}}{\gamma_{\nu_{1}, \nu_{2}}}=\frac{\lambda_{\nu_{1}, \nu_{2}}}{\lambda_{2^{n_{1}-1}, 2^{n_{2}-1}}} \text { for } 2^{n_{1}-1} \leqslant \nu_{1} \leqslant 2^{n_{1}}-1, \\
& 2^{n_{2}-1} \leqslant \nu_{2} \leqslant 2^{n_{2}}-1\left(n_{1}, n_{2}=2,3, \ldots\right) .
\end{aligned}
$$

Let us show that $\left\{\Lambda_{n_{1}, n_{2}}\right\}$ satisfies the conditions of Lemma 3.2.6. Taking into account that $\left\{\lambda_{n_{1}, n_{2}}\right\}=\lambda \in G M^{2}$, we have

1) $\left|\Lambda_{n_{1}, n_{2}}\right| \leqslant M$,

2) for $n_{1}=n_{2}=1$,

$$
\begin{aligned}
& \sum_{\nu_{1}=2^{n_{1}-1}}^{2^{n_{1}}-1}\left|\Lambda_{\nu_{1}, 1}-\Lambda_{\nu_{1}+1,1}\right|=\left|1-\frac{\lambda_{2,1}}{\lambda_{2,1}}\right| \leqslant M, \\
& \sum_{\nu_{2}=2^{n_{2}-1}}^{2_{2}-1}\left|\Lambda_{1, \nu_{2}}-\Lambda_{1, \nu_{2}+1}\right| \leqslant M,
\end{aligned}
$$

3) for $n_{2}=1, \quad n_{1}=2,3, \ldots$,

$$
\sum_{\nu_{1}=2^{n_{1}-1}}^{2^{n_{1}}-1}\left|\Lambda_{\nu_{1}, n_{2}}-\Lambda_{\nu_{1}+1, n_{2}}\right|=\frac{1}{\lambda_{2^{n_{1}-1}, 1}} \sum_{\nu_{1}=2^{n_{1}}-1}^{2^{n_{1}}-2}\left|\lambda_{\nu_{1}}-\lambda_{\nu_{1}+1,1}\right|+\left|\frac{\lambda_{2^{n_{1}}-1,1}}{\lambda_{2^{n_{1}}-1}, 1}-1\right| \leqslant M
$$

4) for $n_{1}=1, n_{2}=2,3, \ldots$,

$$
\sum_{\nu_{2}=2^{n_{2}-1}}^{2^{n_{2}-1}}\left|\Lambda_{1, \nu_{2}}-\Lambda_{1, \nu_{2}+1}\right| \leqslant M
$$

5) for $n_{2}=2,3, \ldots$ such that $2^{m_{2}-1} \leqslant n_{2} \leqslant 2^{m_{2}}-1 \quad\left(m_{2}=2,3, \ldots\right)$,

$$
\begin{aligned}
& \sum_{\nu_{1}=2^{n_{1}-1}}^{2^{n_{1}}-1}\left|\Lambda_{\nu_{1}, n_{2}}-\Lambda_{\nu_{1}+1, n_{2}}\right| \leqslant \frac{1}{\lambda_{2^{n_{1}-1}, 2^{m_{2}-1}}} \sum_{\nu_{1}=2^{n_{1}-1}}^{2^{n_{1}}-2}\left|\lambda_{\nu_{1}, n_{2}}-\lambda_{\nu_{1}+1, n_{2}}\right| \\
& +\frac{\lambda_{2^{n_{1}}-1, n_{2}}}{\lambda_{2^{n_{1}-1}, 2^{m_{2}-1}}}+\frac{\lambda_{2^{n_{1}}, n_{2}}}{\lambda_{2^{n_{1}}, 2^{m_{2}-1}}} \leqslant M,
\end{aligned}
$$


6 ) for $n_{1}=2,3, \ldots$ such that $2^{m_{1}-1} \leqslant n_{1} \leqslant 2^{m_{1}}-1\left(m_{1}=2,3, \ldots\right)$,

$$
\sum_{\nu_{2}=2^{n_{2}-1}}^{2^{n_{2}}-1}\left|\Lambda_{n_{1}, \nu_{2}}-\Lambda_{n_{1}, \nu_{2}+1}\right| \leqslant M
$$

7) for $n_{1}=n_{2}=1$,

$$
\sum_{\nu_{1}=2^{n_{1}-1}}^{2^{n_{1}}-1} \sum_{\nu_{2}=2^{n_{2}-1}}^{2^{n_{2}-1}}\left|\Lambda_{\nu_{1}, \nu_{2}}-\Lambda_{\nu_{1}+1, \nu_{2}}-\Lambda_{\nu_{1}, \nu_{2}+1}+\Lambda_{\nu_{1}+1, \nu_{2}+1}\right| \leqslant M
$$

8) for $n_{1}=1, n_{2}=2,3, \ldots$,

$$
\begin{aligned}
& \sum_{\nu_{1}=2^{n_{1}-1}}^{2^{n_{1}}-1} \sum_{\nu_{2}=2^{n_{2}-1}}^{2^{n_{2}}-1}\left|\Lambda_{\nu_{1}, \nu_{2}}-\Lambda_{\nu_{1}+1, \nu_{2}}-\Lambda_{\nu_{1}, \nu_{2}+1}+\Lambda_{\nu_{1}+1, \nu_{2}+1}\right| \\
& \leqslant \sum_{\nu_{2}=2^{n_{2}-1}}^{2^{n_{2}-1}}\left|\Lambda_{1, \nu_{2}}-\Lambda_{1, \nu_{2}+1}\right|+\sum_{\nu_{2}=2^{n_{2}-1}}^{2^{n_{2}-1}}\left|\Lambda_{2, \nu_{2}}-\Lambda_{2, \nu_{2}+1}\right| \leqslant M,
\end{aligned}
$$

9) for $n_{2}=1, n_{1}=2,3, \ldots$,

$$
\sum_{\nu_{1}=2^{n_{1}-1}}^{2^{n_{1}}-1} \sum_{\nu_{2}=2^{n_{2}}-1}^{2^{n_{2}}-1}\left|\Lambda_{\nu_{1}, \nu_{2}}-\Lambda_{\nu_{1}+1, \nu_{2}}-\Lambda_{\nu_{1}, \nu_{2}+1}+\Lambda_{\nu_{1}+1, \nu_{2}+1}\right| \leqslant M
$$

10) for $n_{1}, n_{2}=2,3, \ldots$,

$$
\begin{aligned}
& \sum_{\nu_{1}=2^{n_{1}-1}}^{2^{n_{1}}-1} \sum_{\nu_{2}=2^{n_{2}-1}}^{2^{n_{2}}-1}\left|\Lambda_{\nu_{1}, \nu_{2}}-\Lambda_{\nu_{1}+1, \nu_{2}}-\Lambda_{\nu_{1}, \nu_{2}+1}+\Lambda_{\nu_{1}+1, \nu_{2}+1}\right| \\
& =\sum_{\nu_{2}=2^{n_{2}-1}}^{2^{n_{2}}-2} \sum_{\nu_{1}=2^{n_{1}-1}}^{2^{n_{1}}-2}\left|\Lambda_{\nu_{1}, \nu_{2}}-\Lambda_{\nu_{1}+1, \nu_{2}}-\Lambda_{\nu_{1}, \nu_{2}+1}+\Lambda_{\nu_{1}+1, \nu_{2}+1}\right| \\
& +\sum_{\nu_{2}=2^{n_{2}-1}}^{2^{n_{2}}-2}\left|\Lambda_{2^{n_{1}}-1, \nu_{2}}-\Lambda_{2^{n_{1}, \nu_{2}}}-\Lambda_{2^{n_{1}}-1, \nu_{2}+1}+\Lambda_{2^{n_{1}, \nu_{2}+1}}\right| \\
& +\sum_{\nu_{1}=2^{n_{1}-2}}^{2^{n_{1}}-2}\left|\Lambda_{\nu_{1}, 2^{n_{2}}-1}-\Lambda_{\nu_{1}+1,2^{n_{2}}-1}-\Lambda_{\nu_{1}, 2^{n_{2}}}+\Lambda_{\nu_{1}+1,2^{n_{2}}}\right| \\
& +\left|\Lambda_{2^{n_{1}}-1,2^{n_{2}-1}}-\Lambda_{2^{n_{2}}, 2^{n_{2}-1}}-\Lambda_{2^{n_{1}}-1,2^{n_{2}}}+\Lambda_{2^{n_{1}}, 2^{n_{2}}}\right| \\
& =\sum_{\nu_{2}=2^{n_{2}-1}}^{2^{n_{2}}-2} \sum_{\nu_{1}=2^{n_{1}-1}}^{2^{n_{1}}-2}\left|\frac{\lambda_{\nu_{1}, \nu_{2}}}{\lambda_{2^{n_{1}-1}, 2^{n_{2}-1}}}-\frac{\lambda_{\nu_{1}+1, \nu_{2}}}{\lambda_{2^{n_{1}-1}, 2^{n_{2}-1}}}-\frac{\lambda_{\nu_{1}, \nu_{2}+1}}{\lambda_{2^{n_{1}-1}, 2^{n_{2}-1}}}+\frac{\lambda_{\nu_{1}+1, \nu_{2}+1}}{\lambda_{2^{n_{1}-1}, 2^{n_{2}-1}}}\right| \\
& +\sum_{\nu_{2}=2^{n_{2}-1}}^{2^{n_{2}}-2}\left|\frac{\lambda_{2^{n_{1}}-1, \nu_{2}}}{\lambda_{2^{n_{1}-1}, 2^{n_{2}-1}}}-\frac{\lambda_{2^{n_{1}}, \nu_{2}}}{\lambda_{2^{n_{1}}, 2^{n_{2}-1}}}-\frac{\lambda_{2^{n_{1}}-1, \nu_{2}+1}}{\lambda_{2^{n_{1}-1}, 2^{n_{2}-1}}}+\frac{\lambda_{2^{n_{1}}, \nu_{2}+1}}{\lambda_{2^{n_{1}}, 2^{n_{2}-1}}}\right|
\end{aligned}
$$




$$
\begin{aligned}
& +\sum_{\nu_{1}=2^{n_{1}-2}}^{2^{n_{1}}-2}\left|\frac{\lambda_{\nu_{1}, 2^{n_{2}}-1}}{\lambda_{2^{n_{1}-1}, 2^{n_{2}-1}}}-\frac{\lambda_{\nu_{1}+1,2^{n_{2}}-1}}{\lambda_{2^{n_{1}-1}, 2^{n_{2}-1}}}-\frac{\lambda_{\nu_{1}, 2^{n_{2}}}}{\lambda_{2^{n_{1}-1}, 2^{n_{2}}}}+\frac{\lambda_{\nu_{1}+1,2^{n_{2}}}}{\lambda_{2^{n_{1}-1}, 2^{n_{2}}}}\right| \\
& +\left|\frac{\lambda_{2^{n_{1}}-1,2^{n_{2}}-1}}{\lambda_{2^{n_{1}-1}, 2^{n_{2}-1}}}-\frac{\lambda_{2^{n_{1}}, 2^{n_{2}}-1}}{\lambda_{2^{n_{1}}, 2^{n_{2}-1}}}-\frac{\lambda_{2^{n_{1}}-1,2^{n_{2}}}}{\lambda_{2^{n_{1}-1}, 2^{n_{2}}}}+\frac{\lambda_{2^{n_{1}}, 2^{n_{2}}}}{\lambda_{2^{n_{1}}, 2^{n_{2}}}}\right| \\
& \leqslant C \frac{\lambda_{2^{n_{1}-1}, 2^{n_{2}-1}}^{\lambda_{2^{n_{1}-1}, 2^{n_{2}-1}}}+\frac{1}{\lambda_{2^{n_{1}-1}, 2^{n_{2}-1}}} \sum_{\nu_{1}=2^{n_{1}-1}}^{2^{n_{1}}-2}\left|\lambda_{\nu_{1}, 2^{n_{2}-1}}-\lambda_{\nu_{1}+1,2^{n_{2}-1}}\right|}{+\frac{1}{\lambda_{2^{n_{1}-1}, 2^{n_{2}}}} \sum_{\nu_{1}=2^{n_{1}-1}}^{2^{n_{1}}-2}\left|\lambda_{\nu_{1}, 2^{n_{2}}}-\lambda_{\nu_{1}+1,2^{2}}\right|+\frac{1}{\lambda_{2^{n_{1}-1}, 2^{n_{2}-1}}} \sum_{\nu_{2}=2^{n_{2}-1}}^{2^{n_{2}-2}}\left|\lambda_{2^{n_{1}}-1, \nu_{2}}-\lambda_{2^{n_{1}}-1, \nu_{2}+1}\right|} \\
& +\frac{1}{\lambda_{2^{n_{1}}, 2^{n_{2}-1}}} \sum_{\nu_{2}=2^{n_{2}-1}}^{2^{n_{2}-2}}\left|\lambda_{2^{n_{1}}, \nu_{2}}-\lambda_{2^{n_{1}}, \nu_{2}+1}\right| \leqslant M .
\end{aligned}
$$

Since the sequence $\left\{\Lambda_{n_{1} n_{2}}\right\}_{n_{1}=1, n_{2}=1}^{\infty, \infty}$ satisfies the conditions of Lemma 3.2.6, then the series (3.3.8) is the Fourier series of a function $f^{\left(\lambda, \beta_{1}, \beta_{2}\right)}\left(x_{1}, x_{2}\right) \in L_{p}$ and $\left\|f^{\left(\lambda, \beta_{1}, \beta_{2}\right)}\right\|_{p} \leqslant C(p, \lambda)\|g\|_{p}$.

Taking into account (3.3.7) and the estimates of $J_{1}, J_{2}, J_{3}$ and $J_{4}$, we have

$$
\begin{aligned}
& \left\|f^{\left(\lambda, \beta_{1}, \beta_{2}\right)}\right\|_{p} \lesssim\left\{\lambda_{1,1}^{\theta}\|f\|_{p}^{\theta}+\sum_{\nu_{1}=1}^{\infty}\left|\lambda_{2^{\nu_{1}}, 1}^{\theta}-\lambda_{2^{\nu_{1}-1}, 1}^{\theta}\right| Y_{2^{\nu_{1}}-1,0}^{\theta}(f)_{p}+\right. \\
& +\sum_{\nu_{2}=1}^{\infty}\left|\lambda_{1,2^{\nu_{2}}}^{\theta}-\lambda_{1,2^{\nu_{2}-1}}^{\theta}\right| Y_{0,2^{\nu_{2}-1}}^{\theta}(f)_{p} \\
& \left.+\sum_{\nu_{2}=1}^{\infty} \sum_{\nu_{1}=1}^{\infty}\left|\lambda_{2^{\nu_{1}}, 2^{\nu_{2}}}^{\theta}-\lambda_{2^{\nu_{1}-1}, 2^{\nu_{2}}}^{\theta}-\lambda_{2^{\nu_{1}}, 2^{\nu_{2}-1}}^{\theta}+\lambda_{2^{\nu_{1}-1}, 2^{\nu_{2}-1}}^{\theta}\right| Y_{2^{\nu_{1}}-1,2^{\nu_{2}-1}}^{\theta}(f)_{p}\right\}^{\frac{1}{\theta}} \\
& \lesssim\left\{\lambda_{1,1}^{\theta}\|f\|_{p}^{\theta}+\sum_{\nu_{1}=1}^{\infty} Y_{2^{\nu_{1}}-1,0}^{\theta}(f)_{p} \sum_{n_{1}=2^{\nu_{1}-1}}^{2^{\nu_{1}}-1}\left|\lambda_{n_{1}+1,1}^{\theta}-\lambda_{n_{1}, 1}^{\theta}\right|\right. \\
& +\sum_{\nu_{2}=1}^{\infty} Y_{0,2^{\nu_{2}-1}}^{\theta}(f)_{p} \sum_{n_{2}=2^{\nu_{2}-1}}^{\infty}\left|\lambda_{1, n_{2}+1}^{\theta}-\lambda_{1, n_{2}}^{\theta}\right| \\
& +\sum_{\nu_{2}=1}^{\infty} \sum_{\nu_{1}=1}^{\infty} Y_{2^{\nu_{1}}-1,2^{\nu_{2}-1}}^{\theta}(f)_{p}
\end{aligned}
$$




$$
\begin{aligned}
& \left.\times \sum_{n_{1}=2^{\nu_{1}-1}}^{2^{\nu_{1}}-1} \sum_{n_{2}=2^{\nu_{2}-1}}^{2^{\nu_{2}}-1}\left|\lambda_{n_{1}, n_{2}}^{\theta}-\lambda_{n_{1}+1, n_{2}}^{\theta}-\lambda_{n_{1}, n_{2}+1}^{\theta}+\lambda_{n_{1}+1, n_{2}+1}^{\theta}\right|\right\}^{\frac{1}{\theta}} \\
& \lesssim\left\{\lambda_{1,1}^{\theta}\|f\|_{p}^{\theta}+\sum_{n_{1}=1}^{\infty}\left|\lambda_{n_{1}+1,1}^{\theta}-\lambda_{n_{1}, 1}^{\theta}\right| Y_{n_{1}, 0}^{\theta}(f)_{p}\right. \\
& +\sum_{n_{2}=1}^{\infty}\left|\lambda_{1, n_{2}+1}^{\theta}-\lambda_{1, n_{2}}^{\theta}\right| Y_{0, n_{2}}^{\theta}(f)_{p} \\
& \left.+\sum_{n_{1}=1}^{\infty} \sum_{n_{2}=1}^{\infty}\left|\lambda_{n_{1}, n_{2}}^{\theta}-\lambda_{n_{1}+1, n_{2}}^{\theta}-\lambda_{n_{1}, n_{2}+1}^{\theta}+\lambda_{n_{1}+1, n_{2}+1}^{\theta}\right| Y_{n_{1}, n_{2}}^{\theta}(f)_{p}\right\}^{\frac{1}{\theta}} .
\end{aligned}
$$

Let us estimate $Y_{2^{m_{1}-1,2^{m_{2}-1}}}\left(f^{\left(\lambda, \beta_{1}, \beta_{2}\right)}\right)_{p}$. Using Lemma 3.2.4, we get

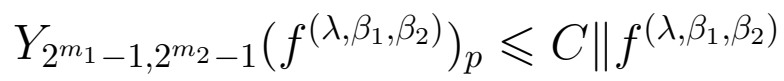

$$
\begin{aligned}
& -S_{\infty, 2^{m_{2}-1}}\left(f^{\left(\lambda, \beta_{1}, \beta_{2}\right)}\right)-S_{2^{m_{1}-1, \infty}}\left(f^{\left(\lambda, \beta_{1}, \beta_{2}\right)}\right)+S_{2^{m_{1}-1,2^{m_{2}-1}}}\left(f^{\left(\lambda, \beta_{1}, \beta_{2}\right)}\right) \|_{p} \text {. }
\end{aligned}
$$

We consider the series (cf. (3.3.8))

$$
\sum_{n_{1}=1}^{\infty} \sum_{n_{2}=1}^{\infty} \lambda_{n_{1}, n_{2}} A_{n_{1} n_{2}}^{*}\left(x_{1}, x_{2}\right)=\sum_{n_{1}=1}^{\infty} \sum_{n_{2}=1}^{\infty} \gamma_{n_{1}, n_{2}} \Lambda_{n_{1}, n_{2}} A_{n_{1} n_{2}}^{*}\left(x_{1}, x_{2}\right),
$$

where $A_{n_{1}, n_{2}}^{*}\left(x_{1}, x_{2}\right)=0$, if $n_{1} \leqslant 2^{m_{1}}-1$ and $n_{2} \leqslant 2^{m_{2}}-1$, also $A_{n_{1}, n_{2}}^{*}\left(x_{1}, x_{2}\right)=$ $A_{n_{1}, n_{2}}\left(x_{1}, x_{2}\right)$ otherwise.

Since the sequence $\left\{\Lambda_{n_{1}, n_{2}}\right\}$ satisfies the conditions of Lemma 3.2.6, then

$$
\left\|\sum_{n_{1}=1}^{\infty} \sum_{n_{2}=1}^{\infty} \lambda_{n_{1}, n_{2}} A_{n_{1} n_{2}}^{*}\left(x_{1}, x_{2}\right)\right\|_{p} \leqslant C\left\|\sum_{n_{1}=1}^{\infty} \sum_{n_{2}=1}^{\infty} \lambda_{2^{n_{1}-1}, 2^{n_{2}-1}} \Delta_{n_{1}, n_{2}}^{*}\right\|_{p},
$$

where $\Delta_{n_{1}, n_{2}}^{*}=0$, if $n_{1} \leqslant m_{1}$ or $n_{2} \leqslant m_{2}, \Delta_{n_{1}, n_{2}}^{*}=\Delta_{n_{1}, n_{2}}$ otherwise.

By Lemma 3.2.5, we have

$Y_{2^{m_{1}}-1,2^{m_{2}-1}}\left(f^{\left(\lambda, \beta_{1}, \beta_{2}\right)}\right)_{p} \lesssim\left\{\int_{0}^{2 \pi} \int_{0}^{2 \pi}\left[\sum_{k_{1}=m_{1}+1}^{\infty} \sum_{k_{2}=m_{2}+1}^{\infty} \lambda_{2^{k_{1}-1}, 2^{k_{2}-1}}^{2} \Delta_{k_{1}, k_{2}}^{2}\right]^{\frac{p}{2}} d x_{1} d x_{2}\right\}^{\frac{1}{p}}$.

It is easy to see that 


$$
\begin{aligned}
& \lambda_{2^{k_{1}-1}, 2^{k_{2}-1}}^{\theta}=\lambda_{2^{m_{1}-1}, 2^{m_{2}-1}}^{\theta}+\sum_{\nu_{2}=m_{2}+1}^{k_{2}}\left(\lambda_{2^{m_{1}-1}, 2^{\nu_{2}-1}}^{\theta}-\lambda_{2^{m_{1}-1}, 2^{\nu_{2}-2}}^{\theta}\right) \\
& +\sum_{\nu_{1}=m_{1}+1}^{k_{1}}\left(\lambda_{2^{\nu_{1}-1}, 2^{m_{2}-1}}^{\theta}-\lambda_{2^{\nu_{1}-2}, 2^{m_{2}-1}}^{\theta}\right) \\
& +\sum_{\nu_{1}=m_{1}+1}^{k_{1}} \sum_{\nu_{2}=m_{2}+1}^{k_{2}}\left[\lambda_{2^{\nu_{1}-1}, 2^{\nu_{2}-1}}^{\theta}-\lambda_{2^{\nu_{1}-2}, 2^{\nu_{2}-1}}^{\theta}-\lambda_{2^{\nu_{1}-1}, 2^{\nu_{2}-2}}^{\theta}+\lambda_{2^{\nu_{1}-2}, 2^{\nu_{2}-2}}^{\theta}\right] \text {. }
\end{aligned}
$$

Substituting this estimate in (3.3.9), we derive

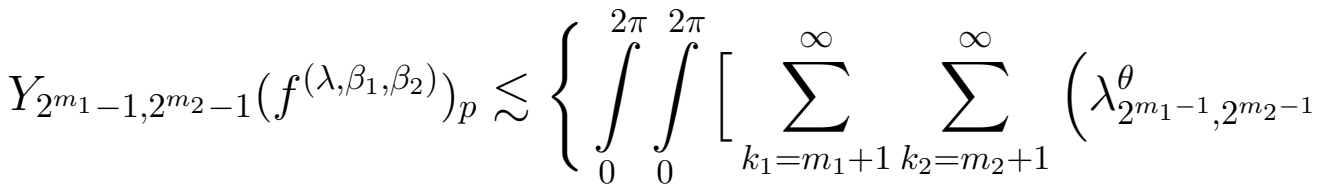

$$
\begin{aligned}
& +\sum_{\nu_{2}=m_{2}+1}^{k_{2}}\left|\lambda_{2^{m_{1}-1}, 2^{\nu_{2}-1}}^{\theta}-\lambda_{2^{m_{1}-1}, 2^{\nu_{2}-2}}^{\theta}\right|+\sum_{\nu_{1}=m_{1}+1}^{k_{1}}\left|\lambda_{2^{\nu_{1}-1}, 2^{m_{2}-1}}^{\theta}-\lambda_{2^{\nu_{1}-2}, 2^{m_{2}-1}}^{\theta}\right| \\
& +\sum_{\nu_{1}=m_{1}+1}^{k_{1}} \sum_{\nu_{2}=m_{2}+1}^{k_{2}} \mid \lambda_{2^{\nu_{1}-1}, 2^{\nu_{2}-1}}^{\theta}-\lambda_{2^{\nu_{1}-2}, 2^{\nu_{2}-1}}^{\theta} \\
& \left.\left.\left.-\lambda_{2^{\nu_{1}-1}, 2^{\nu_{2}-2}}^{\theta}+\lambda_{2^{\nu_{1}-2}, 2^{\nu_{2}-2}}^{\theta} \mid\right)^{\frac{2}{\theta}} \Delta_{k_{1}, k_{2}}^{2}\right]^{\frac{p}{2}} d x_{1} d x_{2}\right\}^{\frac{1}{p}}
\end{aligned}
$$

Then

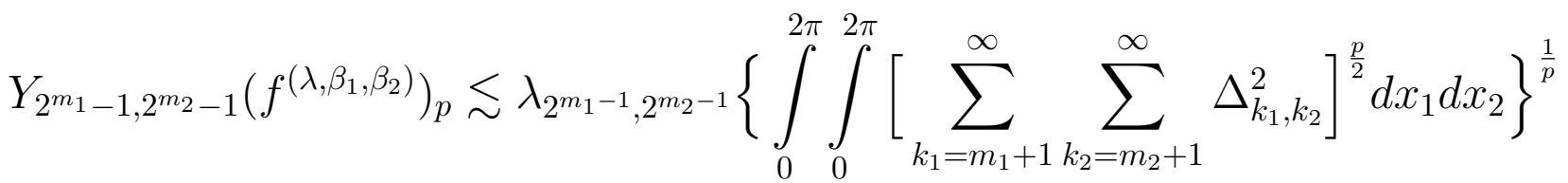

$$
\begin{aligned}
& +\left\{\int_{0}^{2 \pi} \int_{0}^{2 \pi}\left[\sum_{k_{1}=m_{1}+1}^{\infty} \sum_{k_{2}=m_{2}+1}^{\infty} \Delta_{k_{1}, k_{2}}^{2}\left(\sum_{\nu_{1}=m_{1}+1}^{k_{1}}\left|\lambda_{2^{\nu_{1}-1}, 2^{m_{2}-1}}^{\theta}-\lambda_{2^{\nu_{1}-2}, 2^{m_{2}-1}}^{\theta}\right|\right)^{\frac{2}{\theta}}\right]^{\frac{p}{2}} d x_{1} d x_{2}\right\}^{\frac{1}{p}} \\
& +\left\{\int_{0}^{2 \pi} \int_{0}^{2 \pi}\left[\sum_{k_{1}=m_{1}+1}^{\infty} \sum_{k_{2}=m_{2}+1}^{\infty} \Delta_{k_{1}, k_{2}}^{2}\left(\sum_{\nu_{2}=m_{2}+1}^{k_{2}}\left|\lambda_{2^{m_{1}-1}, 2^{\nu_{2}-1}}^{\theta}-\lambda_{2^{m_{1}-1}, 2^{\nu_{2}-2}}^{\theta}\right|\right)^{\frac{2}{\theta}}\right]^{\frac{p}{2}} d x_{1} d x_{2}\right\}^{\frac{1}{p}} \\
& +\left\{\int _ { 0 } ^ { 2 \pi } \int _ { 0 } ^ { 2 \pi } \left[\sum _ { k _ { 1 } = m _ { 1 } + 1 } ^ { \infty } \sum _ { k _ { 2 } = m _ { 2 } + 1 } ^ { \infty } \Delta _ { k _ { 1 } , k _ { 2 } } ^ { 2 } \left(\sum_{\nu_{1}=m_{1}+1}^{k_{1}} \sum_{\nu_{2}=m_{2}+1}^{k_{2}} \mid \lambda_{2^{\nu_{1}-1}, 2^{\nu_{2}-1}}^{\theta}-\lambda_{2^{\nu_{1}-2}, 2^{\nu_{2}-1}}^{\theta}\right.\right.\right.
\end{aligned}
$$




$$
\left.\left.\left.-\lambda_{2^{\nu_{1}-1}, 2^{\nu_{2}-2}}^{\theta}+\lambda_{2^{\nu_{1}-2}, 2^{\nu_{2}-2}}^{\theta} \mid\right)^{\frac{2}{\theta}}\right]^{\frac{p}{2}} d x_{1} d x_{2}\right\}^{\frac{1}{p}}=: L_{1}+L_{2}+L_{3}+L_{4} .
$$

We estimate $L_{1}$ as $J_{1}$ to get

$$
\begin{aligned}
L_{1} & \lesssim \lambda_{2^{m_{1}-1}, 2^{m_{2}-1}}\left\{\int_{0}^{2 \pi} \int_{0}^{2 \pi}\left[\sum_{k_{1}=m_{1}+1}^{\infty} \sum_{k_{2}=m_{2}+1}^{\infty} \Delta_{k_{1}, k_{2}}^{2}\right]^{\frac{p}{2}} d x_{1} d x_{2}\right\}^{\frac{1}{p}} \\
& \lesssim \lambda_{2^{m_{1}-1}, 2^{m_{2}-1}} Y_{2^{m_{1}}-1,2^{m_{2}-1}}(f)_{p} .
\end{aligned}
$$

To estimate $L_{2}$, we have

$$
\begin{aligned}
& \left\{\int_{0}^{2 \pi} \int_{0}^{2 \pi}\left[\sum_{k_{1}=m_{1}+1}^{\infty} \sum_{k_{2}=m_{2}+1}^{\infty} \Delta_{k_{1}, k_{2}}^{2}\left(\sum_{\nu_{1}=m_{1}+1}^{k_{1}}\left|\lambda_{2^{\nu_{1}-1}, 2^{m_{2}-1}}^{\theta}-\lambda_{2^{\nu_{1}-2}, 2^{m_{2}-1}}^{\theta}\right|\right)^{\frac{2}{\theta}}\right]^{\frac{p}{2}} d x_{1} d x_{2}\right\}^{\frac{1}{p}} \\
& \lesssim\left(\sum_{\nu_{1}=m_{1}+1}^{\infty}\left|\lambda_{2^{\nu_{1}-1}, 2^{m_{2}-1}}^{\theta}-\lambda_{2^{\nu_{1}-2}, 2^{m_{2}-1}}^{\theta}\right|\left\{\int_{0}^{2 \pi} \int_{0}^{2 \pi}\left[\sum_{k_{1}=\nu_{1}}^{\infty} \sum_{k_{2}=m_{2}+1}^{\infty} \Delta_{k_{1}, k_{2}}^{2}\right]^{\frac{p}{2}} d x_{1} d x_{2}\right\}^{\frac{\theta}{p}}\right)^{\frac{1}{\theta}} \\
& \lesssim\left(\sum_{\nu_{1}=m_{1}+1}^{\infty}\left|\lambda_{2^{\nu_{1}-1}, 2^{m_{2}-1}}^{\theta}-\lambda_{2^{\nu_{1}-2}, 2^{m_{2}-1}}^{\theta}\right| Y_{2^{\nu_{1}-1}-1,2^{m_{2}-1}}^{\theta}(f)_{p}\right)^{\frac{1}{\theta}} .
\end{aligned}
$$

Similarly, we obtain the estimate for $L_{3}$ :

$$
\begin{aligned}
& \left\{\int_{0}^{2 \pi} \int_{0}^{2 \pi}\left[\sum_{k_{1}=m_{1}+1}^{\infty} \sum_{k_{2}=m_{2}+1}^{\infty} \Delta_{k_{1}, k_{2}}^{2}\left(\sum_{\nu_{2}=m_{2}+1}^{k_{2}}\left|\lambda_{2^{m_{1}-1}, 2^{\nu_{2}-1}}^{\theta}-\lambda_{2^{m_{1}-1}, 2^{\nu_{2}-2}}^{\theta}\right|\right)^{\frac{2}{\theta}}\right]^{\frac{p}{2}} d x_{1} d x_{2}\right\}^{\frac{1}{p}} \\
& \lesssim\left(\sum_{\nu_{2}=m_{2}+1}^{\infty}\left|\lambda_{2^{m_{1}-1}, 2^{\nu_{2}-1}}^{\theta}-\lambda_{2^{m_{1}-1}, 2^{\nu_{2}-2}}^{\theta}\right| Y_{2^{m_{1}-1,2^{\nu_{2}-1}-1}}^{\theta}(f)_{p}\right)^{\frac{1}{\theta}} .
\end{aligned}
$$

Finally, we estimate $L_{4}$ as follows

$$
\begin{aligned}
L_{4}= & \left\{\int _ { 0 } ^ { 2 \pi } \int _ { 0 } ^ { 2 \pi } \left[\sum _ { k _ { 1 } = m _ { 1 } + 1 } ^ { \infty } \sum _ { k _ { 2 } = m _ { 2 } + 1 } ^ { \infty } \Delta _ { k _ { 1 } , k _ { 2 } } ^ { 2 } \left(\sum_{\nu_{1}=m_{1}+1}^{k_{1}} \sum_{\nu_{2}=m_{2}+1}^{k_{2}} \mid \lambda_{2^{\nu_{1}-1}, 2^{\nu_{2}-1}}^{\theta}-\lambda_{2^{\nu_{1}-2}, 2^{\nu_{2}-1}}^{\theta}\right.\right.\right. \\
& \left.\left.\left.-\lambda_{2^{\nu_{1}-1}, 2^{\nu_{2}-2}}^{\theta}+\lambda_{2^{\nu_{1}-2}, 2^{\nu_{2}-2}}^{\theta} \mid\right)^{\frac{2}{\theta}}\right]^{\frac{p}{2}} d x_{1} d x_{2}\right\}^{\frac{1}{p}} \\
& \lesssim\left(\sum_{\nu_{1}=m_{1}+1}^{\infty} \sum_{\nu_{2}=m_{2}+1}^{\infty} \mid \lambda_{2^{\nu_{1}-1}, 2^{\nu_{2}-1}}^{\theta}-\lambda_{2^{\nu_{1}-2}, 2^{\nu_{2}-1}}^{\theta}\right.
\end{aligned}
$$




$$
\begin{aligned}
& \left.\left.-\lambda_{2^{\nu_{1}-1}, 2^{\nu_{2}-2}}^{\theta}+\lambda_{2^{\nu_{1}-2}, 2^{\nu_{2}-2} \mid}^{\theta} \int_{0}^{2 \pi} \int_{0}^{2 \pi}\left[\sum_{k_{1}=\nu_{1}}^{\infty} \sum_{k_{2}=\nu_{2}}^{\infty} \Delta_{k_{1}, k_{2}}^{2}\right]^{\frac{p}{2}} d x_{1} d x_{2}\right\}^{\frac{\theta}{p}}\right)^{\frac{1}{\theta}} \\
& \lesssim\left(\sum_{\nu_{1}=m_{1}+1}^{\infty} \sum_{\nu_{2}=m_{2}+1}^{\infty} \mid \lambda_{2^{\nu_{1}-1}, 2^{\nu_{2}-1}}^{\theta}-\lambda_{2^{\nu_{1}-2}, 2^{\nu_{2}-1}}^{\theta}\right. \\
& \left.-\lambda_{2^{\nu_{1}-1}, 2^{\nu_{2}-2}}^{\theta}+\lambda_{2^{\nu_{1}-2}, 2^{\nu_{2}-2}}^{\theta} \mid Y_{2^{\nu_{1}-1}-1,2^{\nu_{2}-1}-1}^{\theta}(f)_{p}\right)^{\frac{1}{\theta}} .
\end{aligned}
$$

Taking into account the estimates for $L_{1}, L_{2}, L_{3}$, and $L_{4}$, we derive

$$
\begin{aligned}
& Y_{2^{m_{1}-1,2^{m_{2}-1}}}\left(f^{\left(\lambda, \beta_{1}, \beta_{2}\right)}\right)_{p} \lesssim\left\{\lambda_{2^{m_{1}-1}, 2^{m_{2}-1}}^{\theta} Y_{2^{m_{1}-1,2^{m_{2}-1}}}^{\theta}(f)_{p}\right. \\
& +\sum_{\nu_{1}=m_{1}}^{\infty}\left|\lambda_{2^{\nu_{1}, 2^{m_{2}-1}}}^{\theta}-\lambda_{2^{\nu_{1}-1}, 2^{m_{2}-1}}^{\theta}\right| Y_{2^{\nu_{1}-1,2^{m_{2}-1}}}^{\theta}(f)_{p} \\
& +\sum_{\nu_{2}=m_{2}}^{\infty}\left|\lambda_{2^{m_{1}-1}, 2^{\nu_{2}}}^{\theta}-\lambda_{2^{m_{1}-1}, 2^{\nu_{2}-1}}^{\theta}\right| Y_{2^{m_{1}-1,2^{\nu_{2}-1}}}^{\theta}(f)_{p} \\
& +\sum_{\nu_{1}=m_{1}}^{\infty} \sum_{\nu_{2}=m_{2}}^{\infty} \mid \lambda_{2^{\nu_{1}, 2^{\nu_{2}}}}^{\theta}-\lambda_{2^{\nu_{1}-1}, 2^{\nu_{2}}}^{\theta} \\
& \left.-\lambda_{2^{\nu_{1}}, 2^{\nu_{2}-1}}^{\theta}+\lambda_{2^{\nu_{1}-1}, 2^{\nu_{2}-1}}^{\theta} \mid Y_{2^{\nu_{1}-1,2^{\nu_{2}-1}}}^{\theta}(f)_{p}\right\}^{\frac{1}{\theta}} .
\end{aligned}
$$

The proof is now complete.

3.4 Estimates from below of angle best approximations of generalized Liouville-Weyl derivatives

Recall that a sequence $\lambda=\left\{\lambda_{n_{1} n_{2}}\right\}_{n_{1}, n_{2} \in N}$ is such that $\frac{1}{\lambda} \in G M^{2}$ if the relations

$$
\begin{aligned}
& \sum_{k_{1}=n_{1}}^{2 n_{1}}\left|\frac{1}{\lambda_{k_{1}, n_{2}}}-\frac{1}{\lambda_{k_{1}+1, n_{2}}}\right| \leqslant C\left|\frac{1}{\lambda_{n_{1}, n_{2}}}\right|, \\
& \sum_{k_{2}=n_{2}}^{2 n_{2}}\left|\frac{1}{\lambda_{n_{1}, k_{2}}}-\frac{1}{\lambda_{n_{1}, k_{2}+1}}\right| \leqslant C\left|\frac{1}{\lambda_{n_{1}, n_{2}}}\right|,
\end{aligned}
$$




$$
\sum_{k_{1}=n_{1}}^{2 n_{1}} \sum_{k_{2}=n_{2}}^{2 n_{2}}\left|\frac{1}{\lambda_{k_{1}, k_{2}}}-\frac{1}{\lambda_{k_{1}+1, k_{2}}}-\frac{1}{\lambda_{k_{1}, k_{2}+1}}+\frac{1}{\lambda_{k_{1}+1, k_{2}+1}}\right| \leqslant C\left|\frac{1}{\lambda_{n_{1}, n_{2}}}\right|
$$

hold for all integers $n_{1}$ and $n_{2}$, where the constant $C$ is independent of $n_{1}$ and $n_{2}$ (see Definition 3.1.1). Note that the last condition can be equivalently written as follows:

$$
\sum_{k_{1}=n_{1}}^{2 n_{1}} \sum_{k_{2}=n_{2}}^{2 n_{2}}\left|\frac{1}{\lambda_{k_{1}, k_{2}}}-\frac{1}{\lambda_{k_{1}+1, k_{2}}}-\frac{1}{\lambda_{k_{1}, k_{2}+1}}+\frac{1}{\lambda_{k_{1}+1, k_{2}+1}}\right| \leqslant C\left(\left|\frac{1}{\lambda_{n_{1}, n_{2}}}\right|+\left|\frac{1}{\lambda_{2 n_{1}, 2 n_{2}}}\right|\right) \text {. }
$$

Our main result in this subsection is the following analogue of Theorem 1.8.1 in two-dimensional case.

Theorem 3.4.1. Let $1<p<\infty, \max (p, 2) \leqslant \tau<\theta, \alpha_{i} \in \mathbb{R}_{+}, \quad r_{i} \in$ $\mathbb{R}_{+} \cup\{0\}, \beta_{i} \in \mathbb{R}(i=1,2), \lambda=\left\{\lambda_{n_{1}, n_{2}}\right\}_{n_{1}, n_{2} \in N}$ be a sequence of positive numbers such that $\frac{1}{\lambda} \in G M^{2}$, and also satisfy the additional conditions

$$
\begin{aligned}
& \left(\sum_{k_{2}=1}^{n_{2}}\left|\lambda_{2^{n_{1}-1}, 2^{k_{2}}}^{\tau}-\lambda_{2^{n_{1}-1}, 2^{k_{2}-1}}^{\tau}\right|\right)^{\frac{1}{\tau}} \leqslant C\left|\lambda_{2^{n_{1}-1}, 2^{n_{2}}}\right|, \\
& \left(\sum_{k_{1}=1}^{n_{1}}\left|\lambda_{2^{k_{1}}, 2^{n_{2}-1}}^{\tau}-\lambda_{2^{k_{1}-1}, 2^{n_{2}-1}}^{\tau}\right|\right)^{\frac{1}{\tau}} \leqslant C\left|\lambda_{2^{n_{1}}, 2^{n_{2}-1}}\right|, \\
& \left(\sum_{k_{1}=1}^{n_{1}} \sum_{k_{2}=1}^{n_{2}}\left|\lambda_{2^{k_{1}}, 2^{k_{2}}}^{\tau}-\lambda_{2^{k_{1}}, 2^{k_{2}-1}}^{\tau}-\lambda_{2^{k_{1}-1}, 2^{k_{2}}}^{\tau}+\lambda_{2^{k_{1}-1}, 2^{k_{2}-1}}^{\tau}\right|\right)^{\frac{1}{\tau}} \leqslant C\left|\lambda_{2^{n_{1}}, 2^{n_{2}}}\right|,
\end{aligned}
$$

where $n_{1}$ and $n_{2}$ integers and the constant $C$ is independent of $n_{1}$ and $n_{2}$. If $f \in L_{p}^{0}\left(\mathbb{T}^{2}\right)$ and there exists a function $f^{\left(\lambda, \beta_{1}, \beta_{2}\right)} \in L_{p}^{0}\left(\mathbb{T}^{2}\right)$, with the Fourier series $\sigma\left(f, \lambda, \beta_{1}, \beta_{2}\right)$, then

$$
\begin{aligned}
\left\|f^{\left(\lambda, \beta_{1}, \beta_{2}\right)}\right\|_{p} \gtrsim & \left\{\lambda_{1,1}^{\tau}\|f\|_{p}^{\tau}+\sum_{\nu_{1}=1}^{\infty}\left|\lambda_{2^{\nu_{1}, 1}}^{\tau}-\lambda_{2^{\nu_{1}-1}, 1}^{\tau}\right| Y_{2^{\nu_{1}}-1,0}^{\tau}(f)_{p}\right. \\
& +\sum_{\nu_{2}=1}^{\infty}\left|\lambda_{1,2^{\nu_{2}}}^{\tau}-\lambda_{1,2^{\nu_{2}-1}}^{\tau}\right| Y_{0,2^{\nu_{2}}-1}^{\tau}(f)_{p} \\
& \left.+\sum_{\nu_{2}=1}^{\infty} \sum_{\nu_{1}=1}^{\infty}\left|\lambda_{2^{\nu_{1}}, 2^{\nu_{2}}}^{\tau}-\lambda_{2^{\nu_{1}-1}, 2^{\nu_{2}}}^{\tau}-\lambda_{2^{\nu_{1}}, 2^{\nu_{2}-1}}^{\tau}+\lambda_{2^{\nu_{1}-1}, 2^{\nu_{2}-1}}^{\tau}\right| Y_{2^{\nu_{1}}-1,2^{\nu_{2}-1}}^{\tau}(f)_{p}\right\}^{\frac{1}{\tau}}
\end{aligned}
$$


and

$$
\begin{aligned}
& Y_{2^{m_{1}-1,2^{m_{2}-1}}}\left(f^{\left(\lambda, \beta_{1}, \beta_{2}\right)}\right)_{p} \gtrsim\left\{\lambda_{2^{m_{1}-1}, 2^{m_{2}-1}}^{\tau} Y_{2^{m_{1}}-1,2^{m_{2}-1}}^{\tau}(f)_{p}\right. \\
& +\sum_{\nu_{1}=m_{1}}^{\infty}\left|\lambda_{2^{\nu_{1}}, 2^{m_{2}-1}}^{\tau}-\lambda_{2^{\nu_{1}-1}, 2^{m_{2}-1}}^{\tau}\right| Y_{2^{\nu_{1}-1,2^{m_{2}-1}}}^{\tau}(f)_{p} \\
& +\sum_{\nu_{2}=m_{2}}^{\infty}\left|\lambda_{2^{m_{1}-1}, 2^{\nu_{2}}}^{\tau}-\lambda_{2^{m_{1}-1}, 2^{\nu_{2}-1}}^{\tau}\right| Y_{2^{m_{1}}-1,2^{\nu_{2}-1}}^{\tau}(f)_{p} \\
& \left.+\sum_{\nu_{1}=m_{1}}^{\infty} \sum_{\nu_{2}=m_{2}}^{\infty}\left|\lambda_{2^{\nu_{1}}, 2^{\nu_{2}}}^{\tau}-\lambda_{2^{\nu_{1}-1}, 2^{\nu_{2}}}^{\tau}-\lambda_{2^{\nu_{1}, 2^{\nu_{2}-1}}}^{\tau}+\lambda_{2^{\nu_{1}-1}, 2^{\nu_{2}-1}}^{\tau}\right| Y_{2^{\nu_{1}}-1,2^{\nu_{2}-1}}^{\tau}(f)_{p}\right\}^{\frac{1}{\tau}} .
\end{aligned}
$$

Proof of Theorem 3.4.1. We consider the series

$$
\sum_{n_{1}=1}^{\infty} \sum_{n_{2}=1}^{\infty} \lambda_{2^{n_{1}-1}, 2^{n_{2}-1}} \Delta_{n_{1}, n_{2}}=\sum_{n_{1}=1}^{\infty} \sum_{n_{2}=1}^{\infty} \Lambda_{\nu_{1}, \nu_{2}} \lambda_{\nu_{1}, \nu_{2}} A_{\nu_{1}, \nu_{2}}\left(x_{1}, x_{2}\right)
$$

where $\Lambda_{\nu_{1}, \nu_{2}}=\frac{\lambda_{2^{n_{1}-1}, 2^{n_{2}-1}}}{\lambda_{\nu_{1}, \nu_{2}}}$ for $2^{n_{i}-1} \leqslant \nu_{i} \leqslant 2^{n_{i}}-1\left(n_{i}=1,2, \ldots\right), i=1,2$.

Taking into account that $\frac{1}{\lambda} \in G M^{2}$, we list some properties of the sequence $\left\{\Lambda_{\nu_{1}, \nu_{2}}\right\}$ :

1) $\left|\Lambda_{\nu_{1}, \nu_{2}}\right|=\left|\frac{\lambda_{2^{n_{1}-1}, 2^{n_{2}-1}}}{\lambda_{\nu_{1}, \nu_{2}}}\right| \leqslant C\left|\frac{\lambda_{2^{n_{1}-1}, 2^{n_{2}-1}}}{\lambda_{2^{n_{1}-1}, 2^{n_{2}-1}}}\right| \leqslant M$

2) for $n_{1}=n_{2}=1$,

$$
\begin{aligned}
& \sum_{\nu_{1}=2^{n_{1}}-1}^{2^{n_{1}}-1}\left|\Lambda_{\nu_{1}, n_{2}}-\Lambda_{\nu_{1}+1, n_{2}}\right|=\left|\frac{\lambda_{1,1}}{\lambda_{1,1}}-\frac{\lambda_{2,1}}{\lambda_{2,1}}\right| \leqslant M, \\
& \sum_{\nu_{2}=2^{n_{2}-1}}^{2_{2}-1}\left|\Lambda_{n_{1}, \nu_{2}}-\Lambda_{n_{1}, \nu_{2}+1}\right| \leqslant M,
\end{aligned}
$$

3) for $n_{2}=1, n_{1}=2,3, \ldots$,

$$
\begin{aligned}
& \sum_{\nu_{1}=2^{n_{1}-1}}^{2^{n_{1}}-1}\left|\Lambda_{\nu_{1}, n_{2}}-\Lambda_{\nu_{1}+1, n_{2}}\right|=\lambda_{2^{n_{1}-1}, 1} \sum_{\nu_{1}=2^{n_{1}-1}}^{2^{n_{1}}-2}\left|\frac{1}{\lambda_{\nu_{1}, 1}}-\frac{1}{\lambda_{\nu_{1}+1,1}}\right| \\
& +\left|\frac{\lambda_{2^{n_{1}-1}, 1}}{\lambda_{2^{n_{1}}-1,1}}-\frac{\lambda_{2^{n_{1}}, 1}}{\lambda_{2^{n_{1}}, 1}}\right| \leqslant M,
\end{aligned}
$$


4) for $n_{1}=1, n_{2}=2,3, \ldots$,

$\sum_{\nu_{2}=2^{n_{2}-1}}^{2^{n_{2}}-1}\left|\Lambda_{1, \nu_{2}}-\Lambda_{1, \nu_{2}+1}\right| \leqslant M$

5) for $n_{2}=2,3, \ldots: 2^{m_{2}-1} \leqslant n_{2} \leqslant 2^{m_{2}}-1\left(m_{2}=2,3, \ldots\right)$,

$$
\begin{aligned}
& \sum_{\nu_{1}=2^{n_{1}-1}}^{2^{n_{1}}-1}\left|\Lambda_{\nu_{1}, n_{2}}-\Lambda_{\nu_{1}+1, n_{2}}\right| \\
= & \sum_{\nu_{1}=2^{n_{1}-1}}^{2^{n_{1}}-2}\left|\frac{\lambda_{2^{n_{1}-1}, 2^{m_{2}-1}}}{\lambda_{\nu_{1}, n_{2}}}-\frac{\lambda_{2^{n_{1}-1}, 2^{m_{2}-1}}}{\lambda_{\nu_{1}+1, n_{2}}}\right|+\left|\frac{\lambda_{2^{n_{1}-1}, 2^{m_{2}-1}}}{\lambda_{2^{n_{1}}-1, n_{2}}}-\frac{\lambda_{2^{n_{1}}, 2^{m_{2}-1}}}{\lambda_{2^{n_{1}}, n_{2}}}\right| \\
\leqslant & \lambda_{2^{n_{1}-1}, 2^{m_{2}-1}} \sum_{\nu_{1}=2^{n_{1}-1}}^{2^{n_{1}}-2}\left|\frac{1}{\lambda_{\nu_{1}, n_{2}}}-\frac{1}{\lambda_{\nu_{1}+1, n_{2}}}\right|+\frac{\lambda_{2^{n_{1}-1}, 2^{m_{2}-1}}}{\lambda_{2^{n_{1}}-1, n_{2}}}+\frac{\lambda_{2^{n_{1}}, 2^{m_{2}-1}}}{\lambda_{2^{n_{1}}, n_{2}}} \leqslant M,
\end{aligned}
$$

6) for $n_{1}=2,3, \ldots: 2^{m_{1}-1} \leqslant n_{1} \leqslant 2^{m_{1}}-1\left(m_{1}=2,3, \ldots\right)$,

$$
\sum_{\nu_{2}=2^{n_{2}-1}}^{2^{n_{2}-1}}\left|\Lambda_{n_{1}, \nu_{2}}-\Lambda_{n_{1}, \nu_{2}+1}\right| \leqslant M
$$

7) for $n_{1}=n_{2}=1$,

$$
\begin{aligned}
& \sum_{\nu_{1}=2^{n_{1}-1}}^{2^{n_{1}}-1} \sum_{\nu_{2}=2^{n_{2}-1}}^{2^{n_{2}}-1}\left|\Lambda_{\nu_{1}, \nu_{2}}-\Lambda_{\nu_{1}+1, \nu_{2}}-\Lambda_{\nu_{1}, \nu_{2}+1}+\Lambda_{\nu_{1}+1, \nu_{2}+1}\right| \\
= & \left|1-\frac{\lambda_{2,1}}{\lambda_{2,1}}-\frac{\lambda_{1,2}}{\lambda_{1,2}}+\frac{\lambda_{2,2}}{\lambda_{2,2}}\right| \leqslant M,
\end{aligned}
$$

8) for $n_{1}=1, n_{2}=2,3, \ldots$,

$$
\begin{aligned}
& \sum_{\nu_{1}=2^{n_{1}-1}}^{2^{n_{1}}-1} \sum_{\nu_{2}=2^{n_{2}-1}}^{2^{n_{2}}-1}\left|\Lambda_{\nu_{1}, \nu_{2}}-\Lambda_{\nu_{1}+1, \nu_{2}}-\Lambda_{\nu_{1}, \nu_{2}+1}+\Lambda_{\nu_{1}+1, \nu_{2}+1}\right| \\
& \leqslant \sum_{\nu_{2}=2^{n_{2}-1}}^{2^{n_{2}-1}}\left|\Lambda_{1, \nu_{2}}-\Lambda_{1, \nu_{2}+1}\right|+\sum_{\nu_{2}=2^{n_{2}-1}}^{2^{n_{2}-1}}\left|\Lambda_{2, \nu_{2}}-\Lambda_{2, \nu_{2}+1}\right| \leqslant M,
\end{aligned}
$$

9) for $n_{2}=1, n_{1}=2,3, \ldots$,

$$
\begin{aligned}
& \sum_{\nu_{1}=2^{n_{1}}-1}^{2^{n_{1}}-1} \sum_{\nu_{2}=2^{n_{2}-1}}^{2^{n_{2}}-1}\left|\Lambda_{\nu_{1}, \nu_{2}}-\Lambda_{\nu_{1}+1, \nu_{2}}-\Lambda_{\nu_{1}, \nu_{2}+1}+\Lambda_{\nu_{1}+1, \nu_{2}+1}\right| \\
= & \sum_{\nu_{1}=2^{n_{1}-1}}^{2^{n_{1}}-1}\left|\Lambda_{\nu_{1}, 1}-\Lambda_{\nu_{1}+1,1}-\Lambda_{\nu_{1}, 2}+\Lambda_{\nu_{1}+1,2}\right| \leqslant M,
\end{aligned}
$$


10) for $n_{1}, n_{2}=2,3, \ldots$,

$$
\begin{aligned}
& \sum_{\nu_{1}=2^{n_{1}-1}}^{2^{n_{1}}-1} \sum_{\nu_{2}=2^{n_{2}-1}}^{2^{n_{2}}-1}\left|\Lambda_{\nu_{1}, \nu_{2}}-\Lambda_{\nu_{1}+1, \nu_{2}}-\Lambda_{\nu_{1}, \nu_{2}+1}+\Lambda_{\nu_{1}+1, \nu_{2}+1}\right| \\
& =\sum_{\nu_{2}=2^{n_{2}-1}}^{2^{n_{2}}-2} \sum_{\nu_{1}=2^{n_{1}-1}}^{2^{n_{1}}-2}\left|\frac{\lambda_{2^{n_{1}-1}, 2^{n_{2}-1}}}{\lambda_{\nu_{1}, \nu_{2}}}-\frac{\lambda_{2^{n_{1}-1}, 2^{n_{2}-1}}}{\lambda_{\nu_{1}+1, \nu_{2}}}-\frac{\lambda_{2^{n_{1}-1}, 2^{n_{2}-1}}}{\lambda_{\nu_{1}, \nu_{2}+1}}+\frac{\lambda_{2^{n_{1}-1}, 2^{n_{2}-1}}}{\lambda_{\nu_{1}+1, \nu_{2}+1}}\right| \\
& +\sum_{\nu_{1}=2^{n_{1}-2}}^{2^{n_{1}}-2}\left|\frac{\lambda_{2^{n_{1}-1}, 2^{n_{2}-1}}}{\lambda_{\nu_{1}, 2^{n_{2}}-1}}-\frac{\lambda_{2^{n_{1}-1}, 2^{n_{2}-1}}}{\lambda_{\nu_{1}+1,2^{n_{2}}-1}}-\frac{\lambda_{2^{n_{1}-1}, 2^{n_{2}}}}{\lambda_{\nu_{1}, 2^{n_{2}}}}+\frac{\lambda_{2^{n_{1}-1}, 2^{n_{2}}}}{\lambda_{\nu_{1}+1,2^{n_{2}}}}\right| \\
& +\sum_{\nu_{2}=2^{n_{2}-1}}^{2^{n_{2}-2}}\left|\frac{\lambda_{2^{n_{1}-1}, 2^{n_{2}-1}}}{\lambda_{2^{n_{1}}-1, \nu_{2}}}-\frac{\lambda_{2^{n_{1}}, 2^{n_{2}-1}}}{\lambda_{2^{n_{1}}, \nu_{2}}}-\frac{\lambda_{2^{n_{1}-1}, 2^{n_{2}-1}}}{\lambda_{2^{n_{1}}-1, \nu_{2}+1}}+\frac{\lambda_{2^{n_{1}}, 2^{n_{2}-1}}}{\lambda_{2^{n_{1}}, \nu_{2}+1}}\right| \\
& +\left|\frac{\lambda_{2^{n_{1}-1}, 2^{n_{2}-1}}}{\lambda_{2^{n_{1}}-1,2^{n_{2}}-1}}-\frac{\lambda_{2^{n_{1}}, 2^{n_{2}-1}}}{\lambda_{2^{n_{1}}, 2^{n_{2}}-1}}-\frac{\lambda_{2^{n_{1}-1}, 2^{n_{2}}}}{\lambda_{2^{n_{1}}-1,2^{n_{2}}}}+\frac{\lambda_{2^{n_{1}}, 2^{n_{2}}}}{\lambda_{2^{n_{1}}, 2^{n_{2}}}}\right| \\
& \leqslant C \frac{\lambda_{2^{n_{1}-1}, 2^{n_{2}-1}}}{\lambda_{2^{n_{1}-1}, 2^{n_{2}-1}}}+\lambda_{2^{n_{1}-1}, 2^{n_{2}-1}} \sum_{\nu_{1}=2^{n_{1}-1}}^{2^{n_{1}}-2}\left|\frac{1}{\lambda_{\nu_{1}, 2^{n_{2}}-1}}-\frac{1}{\lambda_{\nu_{1}+1,2^{n_{2}-1}}}\right| \\
& +\lambda_{2^{n_{1}-1}, 2^{n_{2}}} \sum_{\nu_{1}=2^{n_{1}-1}}^{2^{n_{1}}-2}\left|\frac{1}{\lambda_{\nu_{1}, 2^{n_{2}}}}-\frac{1}{\lambda_{\nu_{1}+1,2^{n_{2}}}}\right| \\
& +\lambda_{2^{n_{1}-1}, 2^{n_{2}-1}} \sum_{\nu_{2}=2^{n_{2}-1}}^{2^{n_{2}}-2}\left|\frac{1}{\lambda_{2^{n_{1}}-1, \nu_{2}}}-\frac{1}{\lambda_{2^{n_{1}}-1, \nu_{2}+1}}\right| \\
& +\lambda_{2^{n_{1}}, 2^{n_{2}-1}} \sum_{\nu_{2}=2^{n_{2}-1}}^{2^{n_{2}}-2}\left|\frac{1}{\lambda_{2^{n_{1}}, \nu_{2}}}-\frac{1}{\lambda_{2^{n_{1}}, \nu_{2}+1}}\right| \leqslant M .
\end{aligned}
$$

Since the sequence $\left\{\Lambda_{n_{1}, n_{2}}\right\}_{n_{1}=1, n_{2}=1}^{\infty, \infty}$ satisfies the conditions of Lemma 3.2.6, then the series (3.4.11) is the Fourier series of a function $g\left(x_{1}, x_{2}\right) \in L_{p}$, and $\|g\|_{p} \leqslant C(p)\left\|f^{\left(\lambda, \beta_{1}, \beta_{2}\right)}\right\|_{p}$.

Applying Lemmas 1.5.6 and 3.2.5, we get

$$
\begin{aligned}
& \lambda_{1,1}^{\tau}\|f\|_{p}^{\tau}+\sum_{\nu_{1}=1}^{\infty}\left|\lambda_{2^{\nu_{1}, 1}}^{\tau}-\lambda_{2^{\nu_{1}-1}, 1}^{\tau}\right| Y_{2^{\nu_{1}}-1,0}^{\tau}(f)_{p}+\sum_{\nu_{2}=1}^{\infty}\left|\lambda_{1,2^{\nu_{2}}}^{\tau}-\lambda_{1,2^{\nu_{2}-1}}^{\tau}\right| Y_{0,2^{\nu_{2}-1}}^{\tau}(f)_{p} \\
& +\sum_{\nu_{2}=1}^{\infty} \sum_{\nu_{1}=1}^{\infty}\left|\lambda_{2^{\nu_{1}, 2^{\nu_{2}}}}^{\tau}-\lambda_{2^{\nu_{1}-1}, 2^{\nu_{2}}}^{\tau}-\lambda_{2^{\nu_{1}, 2^{\nu_{2}-1}}}^{\tau}+\lambda_{2^{\nu_{1}-1}, 2^{\nu_{2}-1}}^{\tau}\right| Y_{2^{\nu_{1}-1,2^{\nu_{2}-1}}}^{\tau}(f)_{p}
\end{aligned}
$$




$$
\begin{aligned}
& \lesssim \lambda_{1,1}^{\tau}\|f\|_{p}^{\tau}+\sum_{\nu_{1}=1}^{\infty}\left|\lambda_{2^{\nu_{1}, 1}}^{\tau}-\lambda_{2^{\nu_{1}-1}, 1}^{\tau}\right|\left(\int_{0}^{2 \pi} \int_{0}^{2 \pi}\left[\sum_{n_{1}=\nu_{1}+1}^{\infty} \Delta_{n_{1}, 1}^{2}\right]^{\frac{p}{2}} d x_{1} d x_{2}\right)^{\frac{\tau}{p}} \\
& +\sum_{\nu_{2}=1}^{\infty}\left|\lambda_{1,2^{\nu_{2}}}^{\tau}-\lambda_{1,2^{\nu_{2}-1}}^{\tau}\right|\left(\int_{0}^{2 \pi} \int_{0}^{2 \pi}\left[\sum_{n_{2}=\nu_{2}+1}^{\infty} \Delta_{1, n_{2}}^{2}\right]^{\frac{p}{2}} d x_{1} d x_{2}\right)^{\frac{\tau}{p}} \\
& +\sum_{\nu_{2}=1}^{\infty} \sum_{\nu_{1}=1}^{\infty}\left|\lambda_{2^{\nu_{1}}, 2^{\nu_{2}}}^{\tau}-\lambda_{2^{\nu_{1}-1}, 2^{\nu_{2}}}^{\tau}-\lambda_{2^{\nu_{1}, 2^{\nu_{2}-1}}}^{\tau}+\lambda_{2^{\nu_{1}-1}, 2^{\nu_{2}-1}}^{\tau}\right| \\
& \times\left(\int_{0}^{2 \pi} \int_{0}^{2 \pi}\left[\sum_{n_{1}=\nu_{1}+1}^{\infty} \sum_{n_{2}=\nu_{2}+1}^{\infty} \Delta_{n_{1}, n_{2}}^{2}\right]^{\frac{p}{2}} d x_{1} d x_{2}\right)^{\frac{\tau}{p}} \\
& \lesssim \lambda_{1,1}^{\tau}\|f\|_{p}^{\tau}+\left(\int_{0}^{2 \pi} \int_{0}^{2 \pi}\left\{\sum_{\nu_{1}=1}^{\infty}\left|\lambda_{2^{\nu_{1}, 1}}^{\tau}-\lambda_{2^{\nu_{1}-1}, 1}^{\tau}\right|\left[\sum_{n_{1}=\nu_{1}+1}^{\infty} \Delta_{n_{1}, 1}^{2}\right]^{\frac{\tau}{2}}\right\}^{\frac{p}{\tau}} d x_{1} d x_{2}\right)^{\frac{\tau}{p}} \\
& +\left(\int_{0}^{2 \pi} \int_{0}^{2 \pi}\left\{\sum_{\nu_{2}=1}^{\infty}\left|\lambda_{1,2^{\nu_{2}}}^{\tau}-\lambda_{1,2^{\nu_{2}-1}}^{\tau}\right|\left[\sum_{n_{2}=\nu_{2}+1}^{\infty} \Delta_{1, n_{2}}^{2}\right]^{\frac{\tau}{2}}\right\}^{\frac{p}{\tau}} d x_{1} d x_{2}\right)^{\frac{\tau}{p}} \\
& +\left(\int _ { 0 } ^ { 2 \pi } \int _ { 0 } ^ { 2 \pi } \left\{\sum_{\nu_{2}=1}^{\infty} \sum_{\nu_{1}=1}^{\infty}\left|\lambda_{2^{\nu_{1}}, 2^{\nu_{2}}}^{\tau}-\lambda_{2^{\nu_{1}-1}, 2^{\nu_{2}}}^{\tau}-\lambda_{2^{\nu_{1}}, 2^{\nu_{2}-1}}^{\tau}+\lambda_{2^{\nu_{1}-1}, 2^{\nu_{2}-1}}^{\tau}\right|\right.\right. \\
& \left.\left.\times\left[\sum_{n_{1}=\nu_{1}+1}^{\infty} \sum_{n_{2}=\nu_{2}+1}^{\infty} \Delta_{n_{1}, n_{2}}^{2}\right]^{\frac{\tau}{2}}\right\}^{\frac{p}{\tau}} d x_{1} d x_{2}\right)^{\frac{\tau}{p}} \\
& =\lambda_{1,1}^{\tau}\|f\|_{p}^{\tau}+\left(\int_{0}^{2 \pi} \int_{0}^{2 \pi}\left\{\left\{\sum_{\nu_{1}=1}^{\infty}\left[\sum_{n_{1}=\nu_{1}}^{\infty}\left|\lambda_{2^{\nu_{1}, 1}}^{\tau}-\lambda_{2^{\nu_{1}-1}, 1}^{\tau}\right|^{\frac{2}{\tau}} \Delta_{n_{1}+1,1}^{2}\right]^{\frac{\tau}{2}}\right\}^{\frac{2}{\tau}}\right\}^{\frac{p}{2}} d x_{1} d x_{2}\right)^{\frac{\tau}{p}} \\
& +\left(\int_{0}^{2 \pi} \int_{0}^{2 \pi}\left\{\left\{\sum_{\nu_{2}=1}^{\infty}\left[\sum_{n_{2}=\nu_{2}}^{\infty}\left|\lambda_{1,2^{\nu_{2}}}^{\tau}-\lambda_{1,2^{\nu_{2}-1}}^{\tau}\right|^{\frac{2}{\tau}} \Delta_{1, n_{2}+1}^{2}\right]^{\frac{\tau}{2}}\right\}^{\frac{2}{\tau}}\right\}^{\frac{p}{2}} d x_{1} d x_{2}\right)^{\frac{\tau}{p}} \\
& +\left(\int _ { 0 } ^ { 2 \pi } \int _ { 0 } ^ { 2 \pi } \left\{\sum _ { \nu _ { 2 } = 1 } ^ { \infty } \left\{\left\{\sum _ { \nu _ { 1 } = 1 } ^ { \infty } \left[\sum _ { n _ { 1 } = \nu _ { 1 } } ^ { \infty } \left\{\sum_{n_{2}=\nu_{2}}^{\infty} \mid \lambda_{2^{\nu_{1}}, 2^{\nu_{2}}}^{\tau}-\lambda_{2^{\nu_{1}-1}, 2^{\nu_{2}}}^{\tau}\right.\right.\right.\right.\right.\right. \\
& \left.\left.\left.\left.\left.\left.-\lambda_{2^{\nu_{1}}, 2^{\nu_{2}-1}}^{\tau}+\lambda_{2^{\nu_{1}-1}, 2^{\nu_{2}-1}}^{\tau} \frac{\frac{2}{\tau}}{\tau} \Delta_{n_{1}+1, n_{2}+1}^{2}\right\}\right]^{\frac{\tau}{2}}\right\}^{\frac{2}{\tau}}\right\}^{\frac{\tau}{2}}\right\}^{\frac{p}{\tau}} d x_{1} d x_{2}\right)^{\frac{\tau}{p}}=J_{1} .
\end{aligned}
$$

Using Lemmas 1.5.5 and 1.5.6 for $\tau \geqslant 2$, we obtain 


$$
\begin{aligned}
& J_{1} \lesssim \lambda_{1,1}^{\tau}\|f\|_{p}^{\tau}+\left(\int_{0}^{2 \pi} \int_{0}^{2 \pi}\left\{\sum_{n_{1}=1}^{\infty}\left\{\sum_{\nu_{1}=1}^{n_{1}}\left[\mid \lambda_{2^{\nu_{1}, 1}}^{\tau}-\lambda_{2^{\nu_{1}-1}, 1}^{{ }^{\frac{2}{\tau}}} \Delta_{n_{1}+1,1}^{2}\right]^{\frac{\tau}{2}}\right\}^{\frac{2}{\tau}}\right\}^{\frac{p}{2}} d x_{1} d x_{2}\right)^{\frac{\tau}{p}} \\
& +\left(\int_{0}^{2 \pi} \int_{0}^{2 \pi}\left\{\sum_{n_{2}=1}^{\infty}\left\{\sum_{\nu_{2}=1}^{n_{2}}\left[\left|\lambda_{1,2^{\nu_{2}}}^{\tau}-\lambda_{1,2^{\nu_{2}-1}}^{\tau}\right|^{\frac{2}{\tau}} \Delta_{1, n_{2}+1}^{2}\right]^{\frac{\tau}{2}}\right\}^{\frac{2}{\tau}}\right\}^{\frac{p}{2}} d x_{1} d x_{2}\right)^{\frac{\tau}{p}} \\
& +\left(\int _ { 0 } ^ { 2 \pi } \int _ { 0 } ^ { 2 \pi } \left\{\sum _ { \nu _ { 2 } = 1 } ^ { \infty } \left\{\sum _ { n _ { 1 } = 1 } ^ { \infty } \left\{\sum _ { \nu _ { 1 } = 1 } ^ { n _ { 1 } } \left[\left\{\sum_{n_{2}=\nu_{2}}^{\infty} \mid \lambda_{2^{\nu_{1}, \nu^{\nu_{2}}}}^{\tau}-\lambda_{2^{\nu_{1}-1}, 2^{\nu_{2}}}^{\tau}\right.\right.\right.\right.\right.\right. \\
& \left.\left.\left.\left.\left.\left.-\lambda_{2^{\nu_{1}, 2^{\nu_{2}-1}}}^{\tau}+\left.\lambda_{2^{\nu_{1}-1}, 2^{\nu_{2}-1}}^{\tau}\right|^{\frac{2}{\tau}} \Delta_{n_{1}+1, n_{2}+1}^{2}\right\}\right]^{\frac{\tau}{2}}\right\}^{\frac{2}{\tau}}\right\}^{\frac{\tau}{2}}\right\}^{\frac{p}{\tau}} d x_{1} d x_{2}\right)^{\frac{\tau}{p}}
\end{aligned}
$$

Note that the last term is equal to

$$
\begin{aligned}
& \left(\int _ { 0 } ^ { 2 \pi } \int _ { 0 } ^ { 2 \pi } \left[\left\{\sum _ { \nu _ { 2 } = 1 } ^ { \infty } \left\{\sum _ { n _ { 1 } = 1 } ^ { \infty } \left\{\sum _ { \nu _ { 1 } = 1 } ^ { n _ { 1 } } \left[\sum_{n_{2}=\nu_{2}}^{\infty} \mid \lambda_{2^{\nu_{1}, 2^{\nu_{2}}}}^{\tau}-\lambda_{2^{\nu_{1}-1}, 2^{\nu_{2}}}^{\tau}\right.\right.\right.\right.\right.\right.
\end{aligned}
$$

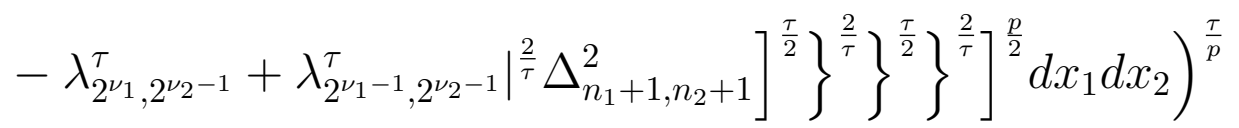

$$
\begin{aligned}
& \lesssim\left(\int _ { 0 } ^ { 2 \pi } \int _ { 0 } ^ { 2 \pi } \left[\sum _ { n _ { 1 } = 1 } ^ { \infty } \left\{\sum _ { \nu _ { 2 } = 1 } ^ { \infty } \left\{\left\{\sum _ { \nu _ { 1 } = 1 } ^ { n _ { 1 } } \left[\sum_{n_{2}=\nu_{2}}^{\infty} \mid \lambda_{2^{\nu_{1}, \nu^{\nu_{2}}}}^{\tau}-\lambda_{2^{\nu_{1}-1}, 2^{\nu_{2}}}^{\tau}\right.\right.\right.\right.\right.\right. \\
& \left.\left.\left.\left.\left.\left.-\lambda_{2^{\nu_{1}, 2^{\nu_{2}-1}}}^{\tau}+\lambda_{2^{\nu_{1}-1}, 2^{\nu_{2}-1}}^{\left.\right|^{\frac{2}{\tau}}} \Delta_{n_{1}+1, n_{2}+1}^{2}\right]^{\frac{\tau}{2}}\right\}^{\frac{2}{\tau}}\right\}^{\frac{\tau}{2}}\right\}^{\frac{2}{\tau}}\right]^{\frac{p}{2}} d x_{1} d x_{2}\right)^{\frac{\tau}{p}} .
\end{aligned}
$$

Applying Minkowskii inequality, we derive the following estimate of the latter:

$$
\begin{aligned}
& \left(\int _ { 0 } ^ { 2 \pi } \int _ { 0 } ^ { 2 \pi } \left[\sum _ { n _ { 1 } = 1 } ^ { \infty } \left\{\sum _ { \nu _ { 2 } = 1 } ^ { \infty } \left\{\sum _ { n _ { 2 } = \nu _ { 2 } } ^ { \infty } \left\{\sum _ { \nu _ { 1 } = 1 } ^ { n _ { 1 } } \left[\mid \lambda_{2^{\nu_{1}}, 2^{\nu_{2}}}^{\tau}-\lambda_{2^{\nu_{1}-1}, 2^{\nu_{2}}}^{\tau}\right.\right.\right.\right.\right.\right. \\
& \left.\left.\left.\left.\left.\left.-\lambda_{2^{\nu_{1}, 2^{\nu_{2}-1}}}^{\tau}+\left.\lambda_{2^{\nu_{1}-1}, 2^{\nu_{2}-1}}\right|^{\frac{2}{\tau}} \Delta_{n_{1}+1, n_{2}+1}^{2}\right]^{\frac{\tau}{2}}\right\}^{\frac{2}{\tau}}\right\}^{\frac{\tau}{2}}\right\}^{\frac{2}{\tau}}\right]^{\frac{p}{2}} d x_{1} d x_{2}\right)^{\frac{\tau}{p}} .
\end{aligned}
$$

Thus,

$$
J_{1} \lesssim \lambda_{1,1}^{\tau}\|f\|_{p}^{\tau}+\left(\int_{0}^{2 \pi} \int_{0}^{2 \pi}\left\{\sum_{n_{1}=1}^{\infty} \Delta_{n_{1}+1,1}^{2}\left\{\sum_{\nu_{1}=1}^{n_{1}}\left|\lambda_{2^{\nu_{1}, 1}}^{\tau}-\lambda_{2^{\nu_{1}-1}, 1}^{\tau}\right|\right\}^{\frac{2}{\tau}}\right\}^{\frac{p}{2}} d x_{1} d x_{2}\right)^{\frac{\tau}{p}}
$$




$$
\begin{aligned}
& +\left(\int_{0}^{2 \pi} \int_{0}^{2 \pi}\left\{\sum_{n_{2}=1}^{\infty} \Delta_{1, n_{2}+1}^{2}\left\{\sum_{\nu_{2}=1}^{n_{2}}\left|\lambda_{1,2^{\nu_{2}}}^{\tau}-\lambda_{1,2^{\nu_{2}-1}}^{\tau}\right|\right\}^{\frac{2}{\tau}}\right\}^{\frac{p}{2}} d x_{1} d x_{2}\right)^{\frac{\tau}{p}} \\
& +\left(\int _ { 0 } ^ { 2 \pi } \int _ { 0 } ^ { 2 \pi } \left[\sum _ { n _ { 1 } = 1 } ^ { \infty } \left\{\sum _ { \nu _ { 2 } = 1 } ^ { \infty } \left\{\sum _ { n _ { 2 } = \nu _ { 2 } } ^ { \infty } \Delta _ { n _ { 1 } + 1 , n _ { 2 } + 1 } ^ { 2 } \left\{\sum_{\nu_{1}=1}^{n_{1}} \mid \lambda_{2^{\nu_{1}}, 2^{\nu_{2}}}^{\tau}-\lambda_{2^{\nu_{1}-1}, 2^{\nu_{2}}}^{\tau}\right.\right.\right.\right.\right. \\
& \left.\left.\left.\left.\left.-\lambda_{2^{\nu_{1}}, 2^{\nu_{2}-1}}^{\tau}+\lambda_{2^{\nu_{1}-1}, 2^{\nu_{2}-1}}^{\tau} \mid\right\}^{\frac{2}{\tau}}\right\}^{\frac{\tau}{2}}\right\}^{\frac{2}{\tau}}\right]^{\frac{p}{2}} d x_{1} d x_{2}\right)^{\frac{\tau}{p}}=J_{2} .
\end{aligned}
$$

By Lemma 1.5.5 and taking into account (3.4.10), we have

$$
\begin{aligned}
& J_{2} \lesssim \lambda_{1,1}^{\tau}\|f\|_{p}^{\tau}+\left(\int_{0}^{2 \pi} \int_{0}^{2 \pi}\left\{\sum_{n_{1}=1}^{\infty} \Delta_{n_{1}+1,1}^{2}\left\{\sum_{\nu_{1}=1}^{n_{1}}\left|\lambda_{2^{\nu_{1}, 1}}^{\tau}-\lambda_{2^{\nu_{1}-1}, 1}^{\tau}\right|\right\}^{\frac{2}{\tau}}\right\}^{\frac{p}{2}} d x_{1} d x_{2}\right)^{\frac{\tau}{p}} \\
& +\left(\int_{0}^{2 \pi} \int_{0}^{2 \pi}\left\{\sum_{n_{2}=1}^{\infty} \Delta_{1, n_{2}+1}^{2}\left\{\sum_{\nu_{2}=1}^{n_{2}}\left|\lambda_{1,2^{\nu_{2}}}^{\tau}-\lambda_{1,2^{\nu_{2}-1}}^{\tau}\right|\right\}^{\frac{2}{\tau}}\right\}^{\frac{p}{2}} d x_{1} d x_{2}\right)^{\frac{\tau}{p}} \\
& +\left(\int _ { 0 } ^ { 2 \pi } \int _ { 0 } ^ { 2 \pi } \left[\sum _ { n _ { 1 } = 1 } ^ { \infty } \sum _ { n _ { 2 } = 1 } ^ { \infty } \Delta _ { n _ { 1 } + 1 , n _ { 2 } + 1 } ^ { 2 } \left\{\sum_{\nu_{2}=1}^{n_{2}} \sum_{\nu_{1}=1}^{n_{1}} \mid \lambda_{2^{\nu_{1}}, 2^{\nu_{2}}}^{\tau}-\lambda_{2^{\nu_{1}-1}, 2^{\nu_{2}}}^{\tau}\right.\right.\right. \\
& \left.\left.\left.-\lambda_{2^{\nu_{1}}, 2^{\nu_{2}-1}}^{\tau}+\lambda_{2^{\nu_{1}-1}, 2^{\nu_{2}-1}}^{\tau} \mid\right\}^{\frac{2}{\tau}}\right]^{\frac{p}{2}} d x_{1} d x_{2}\right)^{\frac{\tau}{p}} \\
& \lesssim \lambda_{1,1}^{\tau}\|f\|_{p}^{\tau}+\left(\int_{0}^{2 \pi} \int_{0}^{2 \pi}\left\{\sum_{n_{1}=1}^{\infty} \Delta_{n_{1}+1,1}^{2} \lambda_{2^{n_{1}, 1}}^{2}\right\}^{\frac{p}{2}} d x_{1} d x_{2}\right)^{\frac{\tau}{p}} \\
& +\left(\int_{0}^{2 \pi} \int_{0}^{2 \pi}\left\{\sum_{n_{2}=1}^{\infty} \Delta_{1, n_{2}+1}^{2} \lambda_{1,2^{n_{2}}}^{2}\right\}^{\frac{p}{2}} d x_{1} d x_{2}\right)^{\frac{\tau}{p}} \\
& +\left(\int_{0}^{2 \pi} \int_{0}^{2 \pi}\left[\sum_{n_{1}=1}^{\infty} \sum_{n_{2}=1}^{\infty} \Delta_{n_{1}+1, n_{2}+1}^{2} \lambda_{2^{n_{1}, 2^{n_{2}}}}^{2}\right]^{\frac{p}{2}} d x_{1} d x_{2}\right)^{\frac{\tau}{p}}=J_{3} .
\end{aligned}
$$

Using Lemma 3.2.5 (a), we obtain

$$
J_{3} \lesssim \lambda_{1,1}^{\tau}\left(\int_{0}^{2 \pi} \int_{0}^{2 \pi}\left\{\sum_{n_{1}=1}^{\infty} \sum_{n_{2}=1}^{\infty} \Delta_{n_{1}, n_{2}}^{2}\right\}^{\frac{p}{2}} d x_{1} d x_{2}\right)^{\frac{\tau}{p}}
$$




$$
\begin{aligned}
& +\left(\int_{0}^{2 \pi} \int_{0}^{2 \pi}\left\{\sum_{n_{1}=2}^{\infty} \Delta_{n_{1}, 1}^{2} \lambda_{2^{n_{1}-1}, 1}^{2}\right\}^{\frac{p}{2}} d x_{1} d x_{2}\right)^{\frac{\tau}{p}} \\
& +\left(\int_{0}^{2 \pi} \int_{0}^{2 \pi}\left\{\sum_{n_{2}=2}^{\infty} \Delta_{1, n_{2}}^{2} \lambda_{1,2^{n_{2}-1}}^{2}\right\}^{\frac{p}{2}} d x_{1} d x_{2}\right)^{\frac{\tau}{p}} \\
& +\left(\int_{0}^{2 \pi} \int_{0}^{2 \pi}\left[\sum_{n_{1}=2}^{\infty} \sum_{n_{2}=2}^{\infty} \Delta_{n_{1}, n_{2}}^{2} \lambda_{2^{n_{1}-1}, 2^{n_{2}-1}}^{2}\right]^{\frac{p}{2}} d x_{1} d x_{2}\right)^{\frac{\tau}{p}} .
\end{aligned}
$$

Similarly to the estimates of $J$ in the proof of Theorem 1.8.1, we derive

$$
\begin{aligned}
& J_{3} \lesssim \lambda_{1,1}^{\tau}\left[\left(\int_{0}^{2 \pi} \int_{0}^{2 \pi}\left\{\Delta_{1,1}^{2}\right\}^{\frac{p}{2}} d x_{1} d x_{2}\right)^{\frac{\tau}{p}}+\left(\int_{0}^{2 \pi} \int_{0}^{2 \pi}\left\{\sum_{n_{1}=2}^{\infty} \Delta_{n_{1}, 1}^{2}\right\}^{\frac{p}{2}} d x_{1} d x_{2}\right)^{\frac{\tau}{p}}\right. \\
& \left.+\left(\int_{0}^{2 \pi} \int_{0}^{2 \pi}\left\{\sum_{n_{2}=2}^{\infty} \Delta_{1, n_{2}}^{2}\right\}^{\frac{p}{2}} d x_{1} d x_{2}\right)^{\frac{\tau}{p}}+\left(\int_{0}^{2 \pi} \int_{0}^{2 \pi}\left\{\sum_{n_{1}=2}^{\infty} \sum_{n_{2}=2}^{\infty} \Delta_{n_{1}, n_{2}}^{2}\right\}^{\frac{p}{2}} d x_{1} d x_{2}\right)^{\frac{\tau}{p}}\right] \\
& +\left(\int_{0}^{2 \pi} \int_{0}^{2 \pi}\left\{\sum_{n_{1}=2}^{\infty} \Delta_{n_{1}, 1}^{2} \lambda_{2^{n_{1}-1}, 1}^{2}\right\}^{\frac{p}{2}} d x_{1} d x_{2}\right)^{\frac{\tau}{p}} \\
& +\left(\int_{0}^{2 \pi} \int_{0}^{2 \pi}\left\{\sum_{n_{2}=2}^{\infty} \Delta_{1, n_{2}}^{2} \lambda_{1,2^{n_{2}-1}}^{2}\right\}^{\frac{p}{2}} d x_{1} d x_{2}\right)^{\frac{\tau}{p}} \\
& +\left(\int_{0}^{2 \pi} \int_{0}^{2 \pi}\left[\sum_{n_{1}=2}^{\infty} \sum_{n_{2}=2}^{\infty} \Delta_{n_{1}, n_{2}}^{2} \lambda_{2^{n_{1}-1}, 2^{n_{2}-1}}^{2}\right]^{\frac{p}{2}} d x_{1} d x_{2}\right)^{\frac{\tau}{p}} \\
& \lesssim\left(\int_{0}^{2 \pi} \int_{0}^{2 \pi}\left[\sum_{n_{1}=1}^{\infty} \sum_{n_{2}=1}^{\infty} \Delta_{n_{1}, n_{2}}^{2} \lambda_{2^{n_{1}-1}, 2^{n_{2}-1}}^{2}\right]^{\frac{p}{2}} d x_{1} d x_{2}\right)^{\frac{\tau}{p}} \lesssim\|g\|_{p}^{\tau} \lesssim\left\|f^{\left(\lambda, \beta_{1}, \beta_{2}\right)}\right\|_{p}^{\tau} .
\end{aligned}
$$

Thus,

$$
\begin{aligned}
& \left\|f^{\left(\lambda, \beta_{1}, \beta_{2}\right)}\right\|_{p} \gtrsim\left\{\lambda_{1,1}^{\tau}\|f\|_{p}^{\tau}+\sum_{\nu_{1}=1}^{\infty}\left|\lambda_{2^{\nu_{1}, 1}}^{\tau}-\lambda_{2^{\nu_{1}-1}, 1}^{\tau}\right| Y_{2^{\nu_{1}-1,0}}^{\tau}(f)_{p}\right. \\
& +\sum_{\nu_{2}=1}^{\infty}\left|\lambda_{1,2^{\nu_{2}}}^{\tau}-\lambda_{1,2^{\nu_{2}-1}}^{\tau}\right| Y_{0,2^{\nu_{2}-1}}^{\tau}(f)_{p}
\end{aligned}
$$




$$
\left.+\sum_{\nu_{2}=1}^{\infty} \sum_{\nu_{1}=1}^{\infty}\left|\lambda_{2^{\nu_{1}}, 2^{\nu_{2}}}^{\tau}-\lambda_{2^{\nu_{1}-1}, 2^{\nu_{2}}}^{\tau}-\lambda_{2^{\nu_{1}, 2^{\nu_{2}-1}}}^{\tau}+\lambda_{2^{\nu_{1}-1}, 2^{\nu_{2}-1}}^{\tau}\right| Y_{2^{\nu_{1}-1,2^{\nu_{2}}-1}}^{\tau}(f)_{p}\right\}^{\frac{1}{\tau}}
$$

Now we estimate $Y_{2^{m_{1}-1,2^{m_{2}-1}}}\left(f^{\left(\lambda, \beta_{1}, \beta_{2}\right)}\right)_{p}$ from below. By Lemma 3.2.5, we have

$$
Y_{2^{m_{1}}-1,2^{m_{2}-1}}\left(f^{\left(\lambda, \beta_{1}, \beta_{2}\right)}\right)_{p} \gtrsim\left\{\int_{0}^{2 \pi} \int_{0}^{2 \pi}\left[\sum_{k_{1}=m_{1}}^{\infty} \sum_{k_{2}=m_{2}}^{\infty} \lambda_{2^{k_{1}}, 2^{k_{2}}}^{2} \Delta_{k_{1}+1, k_{2}+1}^{2}\right]^{\frac{p}{2}} d x_{1} d x_{2}\right\}^{\frac{1}{p}} .
$$

We consider the following series

$$
\begin{aligned}
& J_{4}=\left(\lambda_{2^{m_{1}-1}, 2^{m_{2}-1}}^{\tau} Y_{2^{m_{1}-1,2^{m_{2}-1}}}^{\tau}(f)_{p}\right. \\
& +\sum_{\nu_{1}=m_{1}}^{\infty}\left|\lambda_{2^{\nu_{1}}, 2^{m_{2}-1}}^{\tau}-\lambda_{2^{\nu_{1}-1}, 2^{m_{2}-1}}^{\tau}\right| Y_{2^{\nu_{1}}-1,2^{m_{2}-1}}^{\tau}(f)_{p} \\
& +\sum_{\nu_{2}=m_{2}}^{\infty}\left|\lambda_{2^{m_{1}-1}, 2^{\nu_{2}}}^{\tau}-\lambda_{2^{m_{1}-1}, 2^{\nu_{2}-1}}^{\tau}\right| Y_{2^{m_{1}-1,2^{\nu_{2}-1}}}^{\tau}(f)_{p} \\
& \left.+\sum_{\nu_{1}=m_{1}}^{\infty} \sum_{\nu_{2}=m_{2}}^{\infty}\left|\lambda_{2^{\nu_{1}}, 2^{\nu_{2}}}^{\tau}-\lambda_{2^{\nu_{1}-1}, 2^{\nu_{2}}}^{\tau}-\lambda_{2^{\nu_{1}, 2^{\nu_{2}-1}}}^{\tau}+\lambda_{2^{\nu_{1}-1}, 2^{\nu_{2}-1}}^{\tau}\right| Y_{2^{\nu_{1}-1,2^{\nu_{2}-1}}}^{\tau}(f)_{p}\right)^{\frac{1}{\tau}} .
\end{aligned}
$$

Applying Lemmas 1.5.6 and 3.2.5, we get

$$
\begin{aligned}
& J_{4} \lesssim\left(\lambda_{2^{m_{1}-1}, 2^{m_{2}-1}}^{\tau}\left(\int_{0}^{2 \pi} \int_{0}^{2 \pi}\left[\sum_{n_{1}=m_{1}}^{\infty} \sum_{n_{2}=m_{2}}^{\infty} \Delta_{n_{1}+1, n_{2}+1}^{2}\right]^{\frac{p}{2}} d x_{1} d x_{2}\right)^{\frac{\tau}{p}}\right. \\
& +\sum_{\nu_{1}=m_{1}}^{\infty}\left|\lambda_{2^{\nu_{1}}, 2^{m_{2}-1}}^{\tau}-\lambda_{2^{\nu_{1}-1}, 2^{m_{2}-1}}^{\tau}\right|\left(\int_{0}^{2 \pi} \int_{0}^{2 \pi}\left[\sum_{n_{1}=\nu_{1}}^{\infty} \sum_{n_{2}=m_{2}}^{\infty} \Delta_{n_{1}+1, n_{2}+1}^{2}\right]^{\frac{p}{2}} d x_{1} d x_{2}\right)^{\frac{\tau}{p}} \\
& +\sum_{\nu_{2}=m_{2}}^{\infty}\left|\lambda_{2^{m_{1}-1}, 2^{\nu_{2}}}^{\tau}-\lambda_{2^{m_{1}-1}, 2^{\nu_{2}-1}}^{\tau}\right|\left(\int_{0}^{2 \pi} \int_{0}^{2 \pi}\left[\sum_{n_{1}=m_{1}}^{\infty} \sum_{n_{2}=\nu_{2}}^{\infty} \Delta_{n_{1}+1, n_{2}+1}^{2}\right]^{\frac{p}{2}} d x_{1} d x_{2}\right)^{\frac{\tau}{p}} \\
& +\sum_{\nu_{1}=m_{1}}^{\infty} \sum_{\nu_{2}=m_{2}}^{\infty}\left|\lambda_{2^{\nu_{1}}, 2^{\nu_{2}}}^{\tau}-\lambda_{2^{\nu_{1}-1}, 2^{\nu_{2}}}^{\tau}-\lambda_{2^{\nu_{1}, 2^{\nu_{2}-1}}}^{\tau}+\lambda_{2^{\nu_{1}-1}, 2^{\nu_{2}-1}}^{\tau}\right|
\end{aligned}
$$




$$
\begin{aligned}
& \left.\times\left(\int_{0}^{2 \pi} \int_{0}^{2 \pi}\left[\sum_{n_{1}=\nu_{1}}^{\infty} \sum_{n_{2}=\nu_{2}}^{\infty} \Delta_{n_{1}+1, n_{2}+1}^{2}\right]^{\frac{p}{2}} d x_{1} d x_{2}\right)^{\frac{\tau}{p}}\right)^{\frac{1}{\tau}} \\
& \lesssim\left(\lambda_{2^{m_{1}-1}, 2^{m_{2}-1}}^{\tau}\left(\int_{0}^{2 \pi} \int_{0}^{2 \pi}\left[\sum_{n_{1}=m_{1}}^{\infty} \sum_{n_{2}=m_{2}}^{\infty} \Delta_{n_{1}+1, n_{2}+1}^{2}\right]^{\frac{p}{2}} d x_{1} d x_{2}\right)^{\frac{\tau}{p}}\right. \\
& +\left(\int_{0}^{2 \pi} \int_{0}^{2 \pi}\left\{\sum_{\nu_{1}=m_{1}}^{\infty}\left|\lambda_{2^{\nu_{1}, 2^{m_{2}-1}}}^{\tau}-\lambda_{2^{\nu_{1}-1}, 2^{m_{2}-1}}^{\tau}\right|\left[\sum_{n_{1}=\nu_{1}}^{\infty} \sum_{n_{2}=m_{2}}^{\infty} \Delta_{n_{1}+1, n_{2}+1}^{2}\right]^{\frac{\tau}{2}}\right\}^{\frac{p}{\tau}} d x_{1} d x_{2}\right)^{\frac{\tau}{p}} \\
& +\left(\int_{0}^{2 \pi} \int_{0}^{2 \pi}\left\{\sum_{\nu_{2}=m_{2}}^{\infty}\left|\lambda_{2^{m_{1}-1}, 2^{\nu_{2}}}^{\tau}-\lambda_{2^{m_{1}-1}, 2^{\nu_{2}-1}}^{\tau}\right|\left[\sum_{n_{1}=m_{1}}^{\infty} \sum_{n_{2}=\nu_{2}}^{\infty} \Delta_{n_{1}+1, n_{2}+1}^{2}\right]^{\frac{\tau}{2}}\right\}^{\frac{p}{\tau}} d x_{1} d x_{2}\right)^{\frac{\tau}{p}} \\
& +\left(\int _ { 0 } ^ { 2 \pi } \int _ { 0 } ^ { 2 \pi } \left\{\sum_{\nu_{1}=m_{1}}^{\infty} \sum_{\nu_{2}=m_{2}}^{\infty}\left|\lambda_{2^{\nu_{1}, 2^{\nu_{2}}}}^{\tau}-\lambda_{2^{\nu_{1}-1}, 2^{\nu_{2}}}^{\tau}-\lambda_{2^{\nu_{1}, 2^{\nu_{2}-1}}}^{\tau}+\lambda_{2^{\nu_{1}-1}, 2^{\nu_{2}-1}}^{\tau}\right|\right.\right. \\
& \left.\left.\left.\times\left[\sum_{n_{1}=\nu_{1}}^{\infty} \sum_{n_{2}=\nu_{2}}^{\infty} \Delta_{n_{1}+1, n_{2}+1}^{2}\right]^{\frac{\tau}{2}}\right\}^{\frac{p}{\tau}} d x_{1} d x_{2}\right)^{\frac{\tau}{p}}\right)^{\frac{1}{\tau}} \\
& =\left(\lambda_{2^{m_{1}-1}, 2^{m_{2}-1}}^{\tau}\left(\int_{0}^{2 \pi} \int_{0}^{2 \pi}\left[\sum_{n_{1}=m_{1}}^{\infty} \sum_{n_{2}=m_{2}}^{\infty} \Delta_{n_{1}+1, n_{2}+1}^{2}\right]^{\frac{p}{2}} d x_{1} d x_{2}\right)^{\frac{\tau}{p}}\right. \\
& +\left(\int _ { 0 } ^ { 2 \pi } \int _ { 0 } ^ { 2 \pi } \left\{\sum _ { \nu _ { 1 } = m _ { 1 } } ^ { \infty } \left[\sum_{n_{1}=\nu_{1}}^{\infty} \sum_{n_{2}=m_{2}}^{\infty}\right.\right.\right. \\
& \left.\left.\left.\left.\left|\lambda_{2^{\nu_{1}, 2^{m_{2}-1}}}^{\tau}-\lambda_{2^{\nu_{1}-1}, 2^{m_{2}-1}}^{\tau}\right|^{\frac{2}{\tau}} \Delta_{n_{1}+1, n_{2}+1}^{2}\right]^{\frac{\tau}{2}}\right\}^{\frac{2}{\tau}}\right\}^{\frac{p}{2}} d x_{1} d x_{2}\right)^{\frac{\tau}{p}} \\
& +\left(\int _ { 0 } ^ { 2 \pi } \int _ { 0 } ^ { 2 \pi } \left\{\sum _ { \nu _ { 2 } = m _ { 2 } } ^ { \infty } \left[\sum_{n_{2}=\nu_{2}}^{\infty} \sum_{n_{1}=m_{1}}^{\infty}\right.\right.\right. \\
& \left.\left.\left.\left.\left|\lambda_{2^{m_{1}-1}, 2^{\nu_{2}}}^{\tau}-\lambda_{2^{m_{1}-1}, 2^{\nu_{2}-1}}^{\tau}\right|^{\frac{2}{\tau}} \Delta_{n_{1}+1, n_{2}+1}^{2}\right]^{\frac{\tau}{2}}\right\}^{\frac{2}{\tau}}\right\}^{\frac{p}{2}} d x_{1} d x_{2}\right)^{\frac{\tau}{p}} \\
& +\left(\int _ { 0 } ^ { 2 \pi } \int _ { 0 } ^ { 2 \pi } \left\{\sum _ { \nu _ { 2 } = m _ { 2 } } ^ { \infty } \left\{\left\{\sum _ { \nu _ { 1 } = m _ { 1 } } ^ { \infty } \left[\sum_{n_{1}=\nu_{1}}^{\infty} \sum_{n_{2}=\nu_{2}}^{\infty} \mid \lambda_{2^{\nu_{1}, \nu^{\nu_{2}}}}^{\tau}-\lambda_{2^{\nu_{1}-1}, 2^{\nu_{2}}}^{\tau}\right.\right.\right.\right.\right. \\
& \left.\left.\left.\left.\left.\left.-\lambda_{2^{\nu_{1}, 2^{\nu_{2}-1}}}^{\tau}+\left.\lambda_{2^{\nu_{1}-1}, 2^{\nu_{2}-1}}^{\tau}\right|^{\frac{2}{\tau}} \Delta_{n_{1}+1, n_{2}+1}^{2}\right]^{\frac{\tau}{2}}\right\}^{\frac{2}{\tau}}\right\}^{\frac{\tau}{2}}\right\}^{\frac{p}{\tau}} d x_{1} d x_{2}\right)^{\frac{\tau}{p}}\right)^{\frac{1}{\tau}} \text {. }
\end{aligned}
$$


Further, using Minkowski's inequality and Lemma 1.5.6 for $\tau \geqslant 2$, we obtain

$$
\begin{aligned}
& J_{4} \lesssim\left(\lambda_{2^{m_{1}-1}, 2^{m_{2}-1}}^{\tau}\left(\int_{0}^{2 \pi} \int_{0}^{2 \pi}\left[\sum_{n_{1}=m_{1}}^{\infty} \sum_{n_{2}=m_{2}}^{\infty} \Delta_{n_{1}+1, n_{2}+1}^{2}\right]^{\frac{p}{2}} d x_{1} d x_{2}\right)^{\frac{\tau}{p}}\right. \\
& +\left(\int _ { 0 } ^ { 2 \pi } \int _ { 0 } ^ { 2 \pi } \left\{\sum _ { n _ { 1 } = m _ { 1 } } ^ { \infty } \left\{\sum _ { \nu _ { 1 } = m _ { 1 } } ^ { n _ { 1 } } \left[\sum_{n_{2}=m_{2}}^{\infty}\right.\right.\right.\right. \\
& \left.\left.\left.\left.\left|\lambda_{2^{\nu_{1}}, 2^{m_{2}-1}}^{\tau}-\lambda_{2^{\nu_{1}-1}, 2^{m_{2}-1}}^{\tau}\right|^{\frac{2}{\tau}} \Delta_{n_{1}+1, n_{2}+1}^{2}\right]^{\frac{\tau}{2}}\right\}^{\frac{2}{\tau}}\right\}^{\frac{p}{2}} d x_{1} d x_{2}\right)^{\frac{\tau}{p}} \\
& +\left(\int _ { 0 } ^ { 2 \pi } \int _ { 0 } ^ { 2 \pi } \left\{\sum _ { n _ { 2 } = m _ { 2 } } ^ { \infty } \left\{\sum _ { \nu _ { 2 } = m _ { 2 } } ^ { n _ { 2 } } \left[\sum_{n_{1}=m_{1}}^{\infty}\right.\right.\right.\right. \\
& \left.\left.\left.\left.\mid \lambda_{2^{m_{1}-1}, 2^{\nu_{2}}}^{\tau}-\lambda_{2^{m_{1}-1}, 2^{\nu_{2}-1}}^{\left.\right|^{\frac{2}{\tau}}} \Delta_{n_{1}+1, n_{2}+1}^{2}\right]^{\frac{\tau}{2}}\right\}^{\frac{2}{\tau}}\right\}^{\frac{p}{2}} d x_{1} d x_{2}\right)^{\frac{\tau}{p}} \\
& +\left(\int _ { 0 } ^ { 2 \pi } \int _ { 0 } ^ { 2 \pi } \left\{\sum _ { \nu _ { 2 } = m _ { 2 } } ^ { \infty } \left\{\sum _ { n _ { 1 } = m _ { 1 } } ^ { \infty } \left\{\sum _ { \nu _ { 1 } = m _ { 1 } } ^ { n _ { 1 } } \left[\sum_{n_{2}=\nu_{2}}^{\infty} \mid \lambda_{2^{\nu_{1}}, 2^{\nu_{2}}}^{\tau}-\lambda_{2^{\nu_{1}-1}, 2^{\nu_{2}}}^{\tau}\right.\right.\right.\right.\right. \\
& \left.\left.\left.\left.\left.\left.-\lambda_{2^{\nu_{1}}, 2^{\nu_{2}-1}}^{\tau}+\left.\lambda_{2^{\nu_{1}-1}, 2^{\nu_{2}-1}}^{\tau}\right|^{\frac{2}{\tau}} \Delta_{n_{1}+1, n_{2}+1}^{2}\right]^{\frac{\tau}{2}}\right\}^{\frac{2}{\tau}}\right\}^{\frac{\tau}{2}}\right\}^{\frac{p}{\tau}} d x_{1} d x_{2}\right)^{\frac{\tau}{p}}\right)^{\frac{1}{\tau}} \\
& \lesssim\left(\lambda_{2^{m_{1}-1}, 2^{m_{2}-1}}^{\tau}\left(\int_{0}^{2 \pi} \int_{0}^{2 \pi}\left[\sum_{n_{1}=m_{1}}^{\infty} \sum_{n_{2}=m_{2}}^{\infty} \Delta_{n_{1}+1, n_{2}+1}^{2}\right]^{\frac{p}{2}} d x_{1} d x_{2}\right)^{\frac{\tau}{p}}\right. \\
& +\left(\int _ { 0 } ^ { 2 \pi } \int _ { 0 } ^ { 2 \pi } \left\{\sum_{n_{1}=m_{1}}^{\infty} \sum_{n_{2}=m_{2}}^{\infty} \Delta_{n_{1}+1, n_{2}+1}^{2}\right.\right. \\
& \left.\left.\left\{\sum_{\nu_{1}=m_{1}}^{n_{1}}\left[\left|\lambda_{2^{\nu_{1}}, 2^{m_{2}-1}}^{\tau}-\lambda_{2^{\nu_{1}-1}, 2^{m_{2}-1}}^{\tau}\right|^{\frac{2}{\tau}}\right]^{\frac{\tau}{2}}\right\}^{\frac{2}{\tau}}\right\}^{\frac{p}{2}} d x_{1} d x_{2}\right)^{\frac{\tau}{p}} \\
& +\left(\int _ { 0 } ^ { 2 \pi } \int _ { 0 } ^ { 2 \pi } \left\{\sum_{n_{2}=m_{2}}^{\infty} \sum_{n_{1}=m_{1}}^{\infty}\right.\right. \\
& \left.\left.\left\{\sum_{\nu_{2}=m_{2}}^{n_{2}}\left[\left|\lambda_{2^{m_{1}-1}, 2^{\nu_{2}}}^{\tau}-\lambda_{2^{m_{1}-1}, 2^{\nu_{2}-1}}^{\tau}\right|^{\frac{2}{\tau}} \Delta_{n_{1}+1, n_{2}+1}^{2}\right]^{\frac{\tau}{2}}\right\}^{\frac{2}{\tau}}\right\}^{\frac{p}{2}} d x_{1} d x_{2}\right)^{\frac{\tau}{p}} \\
& +\left(\int _ { 0 } ^ { 2 \pi } \int _ { 0 } ^ { 2 \pi } \left\{\sum _ { \nu _ { 2 } = m _ { 2 } } ^ { \infty } \left\{\sum _ { n _ { 1 } = m _ { 1 } } ^ { \infty } \sum _ { n _ { 2 } = \nu _ { 2 } } ^ { \infty } \left\{\sum _ { \nu _ { 1 } = m _ { 1 } } ^ { n _ { 1 } } \left[\mid \lambda_{2^{\nu_{1}}, 2^{\nu_{2}}}^{\tau}-\lambda_{2^{\nu_{1}-1}, 2^{\nu_{2}}}^{\tau}\right.\right.\right.\right.\right.
\end{aligned}
$$




$$
\begin{aligned}
& \left.\left.\left.\left.\left.\left.-\lambda_{2^{\nu_{1}, 2^{\nu_{2}-1}}}^{\tau}+\left.\lambda_{2^{\nu_{1}-1}, 2^{\nu_{2}-1}}^{\tau}\right|^{\frac{2}{\tau}} \Delta_{n_{1}+1, n_{2}+1}^{2}\right]^{\frac{\tau}{2}}\right\}^{\frac{2}{\tau}}\right\}^{\frac{\tau}{2}}\right\}^{\frac{p}{\tau}} d x_{1} d x_{2}\right)^{\frac{\tau}{p}}\right)^{\frac{1}{\tau}} \\
& \lesssim\left(\lambda_{2^{m_{1}-1}, 2^{m_{2}-1}}^{\tau}\left(\int_{0}^{2 \pi} \int_{0}^{2 \pi}\left[\sum_{n_{1}=m_{1}}^{\infty} \sum_{n_{2}=m_{2}}^{\infty} \Delta_{n_{1}+1, n_{2}+1}^{2}\right]^{\frac{p}{2}} d x_{1} d x_{2}\right)^{\frac{\tau}{p}}\right. \\
& +\left(\int_{0}^{2 \pi} \int_{0}^{2 \pi}\left\{\sum_{n_{1}=m_{1}}^{\infty} \sum_{n_{2}=m_{2}}^{\infty} \Delta_{n_{1}+1, n_{2}+1}^{2}\left\{\sum_{\nu_{1}=m_{1}}^{n_{1}}\left|\lambda_{2^{\nu_{1}, 2^{m_{2}-1}}}^{\tau}-\lambda_{2^{\nu_{1}-1}, 2^{m_{2}-1}}^{\tau}\right|^{\frac{2}{\tau}}\right\}^{\frac{p}{2}} d x_{1} d x_{2}\right)^{\frac{\tau}{p}}\right. \\
& +\left(\int_{0}^{2 \pi} \int_{0}^{2 \pi}\left\{\sum_{n_{2}=m_{2}}^{\infty} \sum_{n_{1}=m_{1}}^{\infty} \Delta_{n_{1}+1, n_{2}+1}^{2}\left\{\sum_{\nu_{2}=m_{2}}^{n_{2}}\left|\lambda_{2^{m_{1}-1}, 2^{\nu_{2}}}^{\tau}-\lambda_{2^{m_{1}-1}, 2^{\nu_{2}-1}}^{\tau}\right|^{\frac{2}{\tau}}\right\}^{\frac{p}{2}} d x_{1} d x_{2}\right)^{\frac{\tau}{p}}\right. \\
& +\left(\int _ { 0 } ^ { 2 \pi } \int _ { 0 } ^ { 2 \pi } \left\{\sum _ { n _ { 2 } = m _ { 2 } } ^ { \infty } \left\{\sum _ { \nu _ { 2 } = m _ { 2 } } ^ { n _ { 2 } } \left\{\sum _ { n _ { 1 } = m _ { 1 } } ^ { \infty } \Delta _ { n _ { 1 } + 1 , n _ { 2 } + 1 } ^ { 2 } \left\{\sum_{\nu_{1}=m_{1}}^{n_{1}} \mid \lambda_{2^{\nu_{1}, 2^{\nu_{2}}}}^{\tau}-\lambda_{2^{\nu_{1}-1}, 2^{\nu_{2}}}^{\tau}\right.\right.\right.\right.\right.
\end{aligned}
$$

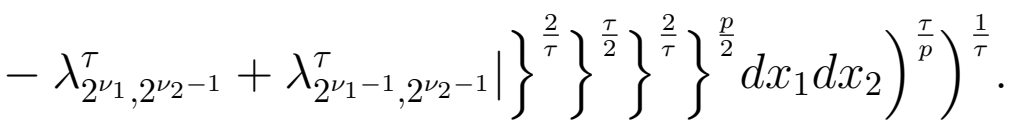

Applying Lemma 1.5.6 and additional conditions (3.4.10), we get

$$
\begin{aligned}
& J_{4} \lesssim\left(\lambda_{2^{m_{1}-1}, 2^{m_{2}-1}}^{\tau}\left(\int_{0}^{2 \pi} \int_{0}^{2 \pi}\left[\sum_{n_{1}=m_{1}}^{\infty} \sum_{n_{2}=m_{2}}^{\infty} \Delta_{n_{1}+1, n_{2}+1}^{2}\right]^{\frac{p}{2}} d x_{1} d x_{2}\right)^{\frac{\tau}{p}}\right. \\
& +\left(\int_{0}^{2 \pi} \int_{0}^{2 \pi}\left\{\sum_{n_{1}=m_{1}}^{\infty} \sum_{n_{2}=m_{2}}^{\infty} \Delta_{n_{1}+1, n_{2}+1}^{2}\left\{\sum_{\nu_{1}=m_{1}}^{n_{1}}\left|\lambda_{2^{\nu_{1}, 2^{m_{2}-1}}}^{\tau}-\lambda_{2^{\nu_{1}-1}, 2^{m_{2}-1}}^{\tau}\right|\right\}^{\frac{2}{\tau}}\right\}^{\frac{p}{2}} d x_{1} d x_{2}\right)^{\frac{\tau}{p}} \\
& +\left(\int_{0}^{2 \pi} \int_{0}^{2 \pi}\left\{\sum_{n_{2}=m_{2}}^{\infty} \sum_{n_{1}=m_{1}}^{\infty} \Delta_{n_{1}+1, n_{2}+1}^{2}\left\{\sum_{\nu_{2}=m_{2}}^{n_{2}}\left|\lambda_{2^{m_{1}-1}, 2^{\nu_{2}}}^{\tau}-\lambda_{2^{m_{1}-1}, 2^{\nu_{2}-1}}^{\tau}\right|^{\frac{2}{\tau}}\right\}^{\frac{p}{2}} d x_{1} d x_{2}\right)^{\frac{\tau}{p}}\right. \\
& +\left(\int _ { 0 } ^ { 2 \pi } \int _ { 0 } ^ { 2 \pi } \left\{\sum _ { n _ { 2 } = m _ { 2 } } ^ { \infty } \sum _ { n _ { 1 } = m _ { 1 } } ^ { \infty } \Delta _ { n _ { 1 } + 1 , n _ { 2 } + 1 } ^ { 2 } \left\{\sum _ { \nu _ { 2 } = m _ { 2 } } ^ { n _ { 2 } } \left\{\left\{\sum_{\nu_{1}=m_{1}}^{n_{1}} \mid \lambda_{2^{\nu_{1}, 2^{\nu_{2}}}}^{\tau}-\lambda_{2^{\nu_{1}-1}, 2^{\nu_{2}}}^{\tau}\right.\right.\right.\right.\right. \\
& \left.\left.\left.\left.\left.\left.-\lambda_{2^{\nu_{1}}, 2^{\nu_{2}-1}}^{\tau}+\lambda_{2^{\nu_{1}-1}, 2^{\nu_{2}-1}}^{\tau} \mid\right\}^{\frac{2}{\tau}}\right\}^{\frac{\tau}{2}}\right\}^{\frac{2}{\tau}}\right\}^{\frac{p}{2}} d x_{1} d x_{2}\right)^{\frac{\tau}{p}}\right)^{\frac{1}{\tau}} \\
& \lesssim\left(\lambda_{2^{m_{1}-1}, 2^{m_{2}-1}}^{\tau}\left(\int_{0}^{2 \pi} \int_{0}^{2 \pi}\left[\sum_{n_{1}=m_{1}+1}^{\infty} \sum_{n_{2}=m_{2}+1}^{\infty} \Delta_{n_{1}, n_{2}}^{2}\right]^{\frac{p}{2}} d x_{1} d x_{2}\right)^{\frac{\tau}{p}}\right.
\end{aligned}
$$




$$
\begin{aligned}
& +\left(\int_{0}^{2 \pi} \int_{0}^{2 \pi}\left\{\sum_{n_{1}=m_{1}+1}^{\infty} \sum_{n_{2}=m_{2}+1}^{\infty} \Delta_{n_{1}, n_{2}}^{2} \lambda_{2^{n_{1}-1}, 2^{m_{2}-1}}^{2}\right\}^{\frac{p}{2}} d x_{1} d x_{2}\right)^{\frac{\tau}{p}} \\
& +\left(\int_{0}^{2 \pi} \int_{0}^{2 \pi}\left\{\sum_{n_{2}=m_{2}+1}^{\infty} \sum_{n_{1}=m_{1}+1}^{\infty} \Delta_{n_{1}, n_{2}}^{2} \lambda_{2^{m_{1}-1}, 2^{n_{2}-1}}^{2}\right\}^{\frac{p}{2}} d x_{1} d x_{2}\right)^{\frac{\tau}{p}} \\
& \left.+\left(\int_{0}^{2 \pi} \int_{0}^{2 \pi}\left\{\sum_{n_{2}=m_{2}+1}^{\infty} \sum_{n_{1}=m_{1}+1}^{\infty} \Delta_{n_{1}, n_{2}}^{2} \lambda_{2^{n_{1}-1}, 2^{n_{2}-1}}^{2}\right\}^{\frac{p}{2}} d x_{1} d x_{2}\right)^{\frac{\tau}{p}}\right)^{\frac{1}{\tau}} .
\end{aligned}
$$

It is easy to see that, for any $n_{1} \geqslant m_{1}$ and $n_{2} \geqslant m_{2}$,

$$
\begin{aligned}
& \lambda_{2^{m_{1}-1}, 2^{m_{2}-1}}^{\tau}=\sum_{\nu_{2}=m_{2}}^{n_{2}-1}\left(\lambda_{2^{m_{1}-1}, 2^{\nu_{2}-1}}^{\tau}-\lambda_{2^{m_{1}-1}, 2^{\nu_{2}}}^{\tau}\right)+\lambda_{2^{m_{1}-1}, 2^{n_{2}}}^{\tau} \\
& \leqslant \sum_{\nu_{2}=m_{2}}^{n_{2}-1}\left|\lambda_{2^{m_{1}-1}, 2^{\nu_{2}-1}}^{\tau}-\lambda_{2^{m_{1}-1}, 2^{\nu_{2}}}^{\tau}\right|+\lambda_{2^{m_{1}-1}, 2^{n_{2}-1}}^{\tau} \\
& \leqslant \sum_{\nu_{2}=m_{2}}^{n_{2}-1}\left|\lambda_{2^{m_{1}-1}, 2^{\nu_{2}-1}}^{\tau}-\lambda_{2^{m_{1}-1}, 2^{\nu_{2}}}^{\tau}\right|+\sum_{\nu_{1}=m_{1}}^{n_{1}-1}\left|\lambda_{2^{\nu_{1}-1}, 2^{n_{2}-1}}^{\tau}-\lambda_{2^{\nu_{1}}, 2^{n_{2}-1}}^{\tau}\right|+\lambda_{2^{n_{1}-1}, 2^{n_{2}-1}}^{\tau} \\
& =\sum_{\nu_{1}=m_{1}}^{n_{1}-1}\left|\lambda_{2^{\nu_{1}-1}, 2^{n_{2}-1}}^{\tau}-\lambda_{2^{\nu_{1}}, 2^{n_{2}-1}}^{\tau}\right|+\lambda_{2^{n_{1}-1}, 2^{n_{2}-1}}^{\tau} \\
& +\sum_{\nu_{2}=m_{2}}^{n_{2}-1} \mid \sum_{\nu_{1}=m_{1}}^{n_{1}-1}\left(\left(\lambda_{2^{\nu_{1}-1}, 2^{\nu_{2}-1}}^{\tau}-\lambda_{2^{\nu_{1}-1}, 2^{\nu_{2}}}^{\tau}\right)-\left(\lambda_{2^{\nu_{1}}, 2^{\nu_{2}-1}}^{\tau}-\lambda_{2^{\nu_{1}}, 2^{\nu_{2}}}^{\tau}\right)\right) \\
& +\left(\lambda_{2^{n_{1}-1}, 2^{\nu_{2}-1}}^{\tau}-\lambda_{2^{n_{1}-1}, 2^{\nu_{2}}}^{\tau}\right) \mid \\
& \leqslant \sum_{\nu_{1}=m_{1}}^{n_{1}-1}\left|\lambda_{2^{\nu_{1}-1}, 2^{n_{2}-1}}^{\tau}-\lambda_{2^{\nu_{1}}, 2^{n_{2}-1}}^{\tau}\right|+\lambda_{2^{n_{1}-1}, 2^{n_{2}-1}}^{\tau} \\
& +\sum_{\nu_{2}=m_{2}}^{n_{2}-1}\left|\sum_{\nu_{1}=m_{1}}^{n_{1}-1}\right|\left(\lambda_{2^{\nu_{1}-1}, 2^{\nu_{2}-1}}^{\tau}-\lambda_{2^{\nu_{1}-1}, 2^{\nu_{2}}}^{\tau}\right)-\left(\lambda_{2^{\nu_{1}}, 2^{\nu_{2}-1}}^{\tau}-\lambda_{2^{\nu_{1}}, 2^{\nu_{2}}}^{\tau}\right) \mid \\
& +\sum_{\nu_{2}=m_{2}}^{n_{2}-1}\left|\lambda_{2^{n_{1}-1}, 2^{\nu_{2}-1}}^{\tau}-\lambda_{2^{n_{1}-1}, 2^{\nu_{2}}}^{\tau}\right| \lesssim \lambda_{2^{n_{1}-1}, 2^{n_{2}-1}}^{\tau}
\end{aligned}
$$

Using this estimate, we derive that 


$$
\begin{aligned}
& J_{4} \lesssim\left(\int_{0}^{2 \pi} \int_{0}^{2 \pi}\left\{\sum_{n_{2}=m_{2}+1}^{\infty} \sum_{n_{1}=m_{1}+1}^{\infty} \Delta_{n_{1}, n_{2}}^{2} \lambda_{2^{n_{1}-1}, 2^{n_{2}-1}}^{2}\right\}^{\frac{p}{2}} d x_{1} d x_{2}\right)^{\frac{1}{p}} \\
& =\left(\int_{0}^{2 \pi} \int_{0}^{2 \pi}\left[\sum_{k_{1}=m_{1}}^{\infty} \sum_{k_{2}=m_{2}}^{\infty} \lambda_{2^{k_{1}, 2^{k_{2}}}}^{2} \Delta_{k_{1}+1, k_{2}+1}^{2}\right]^{\frac{p}{2}} d x_{1} d x_{2}\right)^{\frac{1}{p}} \\
& \lesssim Y_{2^{m_{1}-1,2^{m_{2}-1}}}\left(f^{\left(\lambda, \beta_{1}, \beta_{2}\right)}\right)_{p} \text {. }
\end{aligned}
$$

Thus,

$$
\begin{aligned}
& \left\{\lambda_{2^{m_{1}-1}, 2^{m_{2}-1}}^{\tau} Y_{2^{m_{1}-1,2^{m_{2}-1}}}^{\tau}(f)_{p}+\sum_{\nu_{1}=m_{1}}^{\infty}\left|\lambda_{2^{\nu_{1}, 2^{m_{2}-1}}}^{\tau}-\lambda_{2^{\nu_{1}-1}, 2^{m_{2}-1}}^{\tau}\right| Y_{2^{\nu_{1}-1,2^{m_{2}-1}}}^{\tau}(f)_{p}\right. \\
& +\sum_{\nu_{2}=m_{2}}^{\infty}\left|\lambda_{2^{m_{1}-1}, 2^{\nu_{2}}}^{\tau}-\lambda_{2^{m_{1}-1}, 2^{\nu_{2}-1}}^{\tau}\right| Y_{2^{m_{1}-1,2^{\nu_{2}-1}}}^{\tau}(f)_{p} \\
& \left.+\sum_{\nu_{1}=m_{1}}^{\infty} \sum_{\nu_{2}=m_{2}}^{\infty}\left|\lambda_{2^{\nu_{1}}, 2^{\nu_{2}}}^{\tau}-\lambda_{2^{\nu_{1}-1}, 2^{\nu_{2}}}^{\tau}-\lambda_{2^{\nu_{1}, 2^{\nu_{2}-1}}}^{\tau}+\lambda_{2^{\nu_{1}-1}, 2^{\nu_{2}-1}}^{\tau}\right| Y_{2^{\nu_{1}-1,2^{\nu_{2}-1}}}^{\tau}(f)_{p}\right\}^{\frac{1}{\tau}} \\
& \lesssim Y_{2^{m_{1}-1,2^{m_{2}-1}}}\left(f^{\left(\lambda, \beta_{1}, \beta_{2}\right)}\right)_{p} \text {, }
\end{aligned}
$$

completing the proof. 


\section{Bibliography}

1 N. I. Ahiezer, Lectures in the Theory of Approximation, Nauka, Moscow, 1965. English translation: N.I. Achieser, Theory of Approximation. Dover Publications, New York, 1992.

2 N.K. Bari, S.B. Steckin, Best approximations and differential properties of two conjugate functions, Trans. Moscow Math. Soc., 5 (1956), 483522 .

3 C. Bennett, R. Sharpley, Interpolation of Operators, Academic Press, 1988.

4 S. Bernstein, On the best approximation of continuous functions by polynomials of a given degree, Comm. Soc. Math. Kharkow, Is. 2, Vol. 13 (1912), 49-194.

5 O.V. Besov, On some conditions for derivatives of periodic functions to belong to $L_{p}$, Nauchn. Dokl. Vyssh. Shkoly Fiz.-Mat. Nauki, 1 (1959), 13-17, [in Russian].

6 N.H. Bingham, C.M. Goldie, J.L. Teugels, Regular Variation, Cambridge Univ. Press, 1987.

7 P.L. Butzer, H. Dyckhoff, E. Görlich, R. L. Stens, Best trigonometric approximation, fractional order derivatives and Lipschitz classes, Canad. J. Math., 29 (1977), 781-793.

8 R. DeVore, G.G. Lorentz, Constructive Approximation, Berlin: Springer-Verlag, 1993.

9 R. DeVore, S. Riemenschneider, R. Sharpley, Weak interpolation in Banach spaces, J. Funct. Anal., 33 (1979), 58-94.

10 O. Domínguez, Ulyanov-type inequalities and embeddings between Besov spaces: the case of parameters with limit values, Mathematical Inequalities and Applications, Is. 3, Vol. 20 (2017), 755-772.

11 Z. Ditzian, S. Tikhonov, Moduli of smoothness of functions and their derivatives, Studia Math., 180 (2007), 143-160.

12 Z. Ditzian, S. Tikhonov, Ul'yanov and Nikol'skii-type inequalities, J. Approx. Theory, 133 (2005), 100-133. 
13 Z. Ditzian, V.H. Hristov, K.G. Ivanov, Moduli of smoothness and Kfunctionals in $L_{p}, 0<p<1$, Constructive Approximation, Is. 1, Vol. 11 (1995), 67-83.

14 M. Dyachenko, S. Tikhonov, Convergence of trigonometric series with general monotone coefficients, C.R. Acad. Sci. Paris, Is. 3, Vol. 345 (2007), 123-126.

15 M. Dyachenko, S. Tikhonov, A Hardy-Littlewood theorem for multiple series, J. Math. Anal. Appl., 339 (2008), 503-510.

16 M. Dyachenko, S. Tikhonov, General monotone sequences and convergence of trigonometric series, Topics in Classical Analysis and Applications in Honor of Daniel Waterman, (2008), 88-101.

17 M. Dyachenko, S. Tikhonov, Integrability and continuity of functions represented by trigonometric series: coefficients criteria, Studia mathematica, 193 (2009), 285-306.

18 L. Fejer, Über die Positivat von Summen die nach trigonometrischen oder Legendreschen Funktionen fortschreiten, Acta Lit. Ac. Sci., 2 (1925), 75-86.

19 A. Gogatishvili, B. Opic, S. Tikhonov, W. Trebels, Ulyanov-type inequalities between Lorentz-Zygmund spaces, J. Fourier Anal. Appl., Is. 5, Vol. 20 (2014), 1020-1049.

20 M.L. Gol'dman, Embedding of constructive and structural Lipschitz spaces in symmetric spaces, Tr. Mat. Inst. Steklova, 173 (1986), 90112 (in Russian); translated in: Proc. Steklov Inst. Math., Is. 4, Vol. 173 (1987), 93-118.

21 M.L. Gol'dman, A criterion for the embedding of different metrics for isotropic Besov spaces with arbitrary moduli of continuity, Proc. Steklov Inst. Math., Is. 2, Vol. 201 (1994), 155-181; translation from Trudy Mat. Inst. Steklov., 201 (1992), 186-218.

22 R. Gorenflo, F. Mainardi, Fractional Calculus and Special Functions, in Lecture Notes on Mathematical Physics, University of Bologna(2013), available online at: http://www.fracalmo.org/GORONFLOMAINARDI-SEMINARS/fmfcsf-MaPhySto2000.pdf

23 L.S. Hahn, On multipliers of p-integrable functions, Trans. Amer. Math. Soc., 128 (1967), 321-335.

24 L.S. Hahn, A theorem on multipliers of type $(p, q)$, Proc. Amer. Math. Soc., 21 (1969), 493-495.

25 L. Hörmander, Estimates for translation invariant operators in Lp spaces, Acta Math., 104 (1960), 93-140. 
26 I.I. Hirschman, On multiplier transformations, Duke Math. J., 26 (1959), 221-242.

27 D. Jackson, The theory of approximation, Amer. Math. Soc., 1930.

28 D. Jackson, Über die Genauigkeit der Annäheurung Stetiger Funktionen durch ganze rationale Funtionen gegebenen Grades und trigonometrischen Summen gegebenen Ordnung, Diss., Göttingen University, 1911.

29 H. Johnen, K. Scherer, On the equivalence of the K-functional and moduli of continuity and some applications, Constructive theory of functions of several variables (Proc.Conf., Math.Res.Inst., Oberwolfach, (1976)), Lecture Notes in Math., 571, Springer-Verlag, BerlinHeidelberg, (1977), 119-140.

30 A. Jumabayeva, Liouville-Weyl derivatives, best approximations, and moduli of smoothness, Acta Math. Hungar., Is. 2, Vol. 145 (2015), 369391.

31 A. Jumabayeva, Sharp Ulyanov inequalities for generalized LiouvilleWeyl derivatives, Analysis Math., Is. 2, Vol. 43 (2017), 279-302.

32 A. Jumabayeva, B. Simonov, Liouville-Weyl derivatives of double trigonometric series, Submitted to the proceedings of the AT2017 conference.

33 A. Jumabayeva, B. Simonov, Inequalities for moduli of smoothness of functions and their Liouville-Weyl derivatives, Submitted to Acta Math. Hungar.

34 Y. Kolomoitsev, S. Tikhonov, Hardy-Littlewood and Ulyanov inequalities arXiv preprint arXiv:1711.08163 (2016).

35 Y. Kolomoitsev, Best approximations and moduli of smoothness of functions and their derivatives in $L_{p}, 0<p<1$, arXiv:1612.08020.

36 V.I. Kolyada, On the relations between moduli of continuity in various metrics, Proc. Steklov Inst. Math., 181 (1989), 127-148. Translated from: Studies in the theory of differentiable functions of several variables and its applications, XII (Russian). Tr. Mat. Inst. Steklova, 181 (1988), 117-136.

37 A.A. Konyushkov, Best approximations by trigonometric polynomials and Fourier coefficients, Mat. Sb. (N.S.), Is. 1, Vol. 44(86) (1958), 5384.

38 J.L. Lavoie, T.J. Osler, R. Tremblay, Fractional derivatives and special functions, SIAM Rev., Is. 2, Vol. 18 (1976), 240-268.

39 L. Leindler, On the uniform convergence and boundedness of a certain class of sine series, Anal. Math., 27 (2001), 279-285. 
40 L. Leindler, Embedding relations of classes of numerical sequences, Acta Sci. Math. (Szeged), 68 (2002), 689-695.

41 E. Liflyand, S. Tikhonov, A concept of general monotonicity and application, Math. Nachr., 284, N 8-9, (2011), 1083-1098.

42 J. Marcinkiewicz, Sur quelques intégrales du type de Dini, Ann. Soc. Polon. Math., 17 (1938), 42-50.

43 S.M. Nikolskii, Approximation of Functions of Many Variables and Imbedding Theorems. Nauka, M., 1969. English translation: S.M. Nikolskii, Approximation of Functions of Several Variables and Imbedding Theorems, Springer-Verlag, New York, 1975.

44 M.K. Potapov, On Approximation by "angle", in Proc. Conf. on Constructive Theory of Functions, (Approximation Theory), Budapest, (1969), (Akad. Kiadó, Budapest, 1972), 371-399.

45 M.K. Potapov, B.V. Simonov, S. Tikhonov, Mixed moduli of smoothness in $L_{p}, 1<p<\infty$ : a survey, Surveys in Approximation Theory, 8 (2013), 1-57.

46 M.K. Potapov, B.V. Simonov, S. Tikhonov, Transformation of Fourier series of functions by means power and slowly oscillating, Mat. Zametki, Is. 1, Vol. 77 (2005), 99-116, translation in Math. Notes, Is. 1, Vol. 77 (2005), 90-107.

47 M.K. Potapov, B.V. Simonov, S. Tikhonov, On Besov, Besov-Nikolskii classes and on the estimates of mixed smoothness moduli of fractional derivatives, Trudy Matematicheskogo Instituta imeni V. A. Steklova, 243 (2003), 244-256, translation in Proceedings of the Steklov Institute of Mathematics, 243 (2003), 234-246.

48 M.K. Potapov, B.V. Simonov, S. Tikhonov, Relations between Mixed Moduli of Smoothness and Embedding Theorems for Nikol'skii Classes, Proceedings of the Steklov Institute of Mathematics, 269 (2010), 197207; translation from Russian: Trudy Matem. Inst. V. A. Steklova, 269 (2010), 204-214.

49 M.K. Potapov, B.V. Simonov, S. Tikhonov, Fractional Moduli of Smoothness, Maks Press, Moscow, 2016, [in Russian].

50 R. Salem, Essais sur les Séries Trigonométriques, Actualités scientifiques et industrialles, N 862, Herman, Paris, 1940.

51 R. Salem, Sur les transformations des séries de Fourier, Fund. Math., 33 (1939), 108-114.

52 S.G. Samko, A.A. Kilbas, O.I. Marichev Fractional Integrals and Derivatives., Gordon and Breach Science Publishers, 1993.

53 B.V. Simonov, Some questions in approximation theory and embedding theorems, Diss. Kand. Nauk, (1985), [in Russian]. 
54 B.V. Simonov, S. Tikhonov, On embedings of functional classes defined by constructive characteristics, Banach Center Publications, 72 (2006), 285-307.

55 B.V. Simonov, S. Tikhonov, Embedings theorems in constructive approximation, Sbornik: Mathematics, Is. 9, Vol. 199 (2008), 1367-1407.

56 B.V. Simonov, S. Tikhonov, Sharp Ul'yanov-type inequalities using fractional smoothness, J. Approx. Theory, 162 (2010), 1654-1684.

57 S.M. Shah, A note on quasi-monotone series, Math. Student, 15 (1947), $19-24$.

58 G. Sokolov, Sur quelques propriétés extrémales des sommes trigonomtriques, Bulletin de l'Academie des Sciences de l'URSS., 6-7 (1935), 857-884.

59 S.B. Stechkin, On the order of the best approximations of continuous functions, Izvestiya Akad. Nauk SSSR. Ser. Mat., 15 (1951), 219-242.

60 S.B. Stechkin, On best approximation of conjugate functions by trigonometric polynomials, Izv. Akad. Nauk SSSR, 20 (1956), 197-206.

61 S.B. Stechkin, On the problem of multipliers for trigonometric polynomials, Doklady Akad. Nauk SSSR, 75 (1950), 165-168, [in Russian].

62 S. B. Stechkin, Trigonometric series with monotone type coefficients, Proc. Inst. Math. Mech., 1 (2001), 214-224.

63 O. Szasz, Quasi-monotone series. Amer. J. Math., 70 (1948), 203-206.

64 I. Szalay, On the best approximation of factorized Fourier series, Approximation theory Proc. Conf. Inst. Math. Adam Mickiewicz Univ., Poznań, (1975), 235-241.

65 G. Szegö, Über einen Satz des Herrn Serge Bernstein, Schr. Königsberger Gelehrten Ges. Naturwiss. KI. H., 4 (1928), 59-70.

66 R. Taberski, Differences, moduli and derivatives of fractional orders, Comment. Math. Prace Mat., Is. 2, Vol. 19 (1977), 389-400.

67 S.A. Telyakovskii, S.B. Stechkins works on polynomial approximations of periodic functions, Fundam. Prikl. Mat., Is. 4, Vol. 3 (1997), 10591068, [in Russian].

68 S. Tikhonov, Weak type inequalities for moduli of smoothness: the case of limit value parameters. J. Fourier Anal. Appl., 16 (2010), 590-608.

69 S. Tikhonov, Trigonometric series with general monotone coefficients, J. Math. Anal. Appl., 326 (2007), 721-735. 
70 S. Tikhonov, Embedding results in questions of strong approximation by Fourier series, Acta Sci. Math. (Szeged), 72 (2006), 117-128; published first as S. Tikhonov, Embedding theorems of function classes, IV. November 2005, CRM preprint.

71 S. Tikhonov, Trigonometric series of Nikol'skii classes, Acta Math. Hungar., Is. 1-2, Vol. 114 (2007), 61-78.

72 S. Tikhonov, On moduli of smoothness of fractional order, Real Analysis Exchange, Is. 2, Vol. 30 (2004/05), 507-518.

73 S. Tikhonov, W. Trebels, Ulyanov-type inequalities and generalized Liouville derivatives. Proc. Roy. Soc. Edinburgh Sect. A, Is. 1, Vol. 141 (2011), 205-224.

74 S. Tikhonov, On two theorems of Lorentz, Izv. Ross. Akad. Nauk, Ser. Mat., Is. 1, Vol. 69 (2005), 165-178, translation in Russian Acad. Sci. Izv. Math, Is. 1, Vol. 69 (2005), 163-175.

75 S. Tikhonov, Moduli of smoothness and the interrelation of some classes of functions, Function Spaces, Interpolation Theory and Related Topics: Proc. Conf. on Function Spaces, Interpolation Theory and Related Topics in Honour of Jaak Peetre on his 65th Birthday, August 17-22, 2000. Berlin: W. de Gruyter (2002), 413-423.

76 S. Tikhonov, On generalized Lipschitz classes and Fourier series, Zeitschrift fr Analysis und ihre Anwendungen, Is. 4, Vol. 23 (2004), $745-764$.

77 S. Tikhonov, Generalized Lipschitz classes and Fourier coefficients, Mathematical Notes, 75 (2004), 885-889.

78 A.F. Timan, Theory of Approximation of Functions of a Real Variable, Gosudarstv. Izdat. Fiz.-Mat. Lit., Moscow 1960. English translation: A.F. Timan, Theory of Approximation of Functions of a Real Variable, The Macmillan Co., New York, 1963.

79 M.F. Timan, Inverse theorems of the constructive theory of functions in Lp spaces $(1 \leqslant p \leqslant \infty)$, Mat. Sb. N.S., Is. 88, Vol. 46 (1958), 125-132.

80 M.F. Timan, On Jackson's theorem in Lp-spaces, Ukrain. Mat. Th., Is. 1, Vol. 18 (1966), 134-137.

81 M.F. Timan, Best approximation and modulus of smoothness of functions prescribed on the entire real axis, Izv. Vyss. Ucebn. Zaved. Matematika, Is. 6, Vol. 25 (1961), 108-120.

82 M.F. Timan, The imbedding of the $L_{p}^{(k)}$ classes of functions, Izv. Vyssh. Uchebn. Zaved. Mat., 10 (1974), 61-74.

$83 \mathrm{~W}$. Trebels, Inequalities for moduli of smoothness versus embeddings of function spaces, Arch. Math., 94 (2010), 155-164. 
$84 \mathrm{~W}$. Trebels, Multipliers for $(C, \alpha)$-bounded Fourier expansions in $B a$ nach spaces and approximation theory, Lecture Notes in Mathematics, 329, 1973.

85 P.L. Ul'yanov, The imbedding of certain function classes $H_{p}^{\omega}$. Math. USSR-Izv., 2 (1968), 601-637.

86 P.L. Ul'yanov, Imbedding theorems and relations between best approximations (moduli of continuity) in different metrics, Mat. Sb. (N.S.), Is. 1, Vol. 81 (123) (1970), 104-131.

87 V.V. Zhuk, G.I. Natanson, S.N. Bernstein and direct and converse theorems of constructive function theory, Tr. St.-Peterbg. Mat. Obshch., Vol. 8, Nauchnaya Kniga, Novosibirsk (2000), 70-95; English transl., Amer. Math. Soc. Transl. Ser. 2, vol. 205, Amer. Math. Soc., Providence, RI (2002), 59-82.

88 A. Zygmund, Trigonometric Series, Vol. I. II Third edition. Cambridge 2002 . 